\title{
An integrated approach using patient-specific induced pluripotent stem cells and protein biochemistry to study Vici syndrome associated cardiomyopathy
}

\author{
Doctoral Thesis \\ In partial fulfillment of the requirements for the degree \\ "Doctor rerum naturalium (Dr. rer. nat.)" \\ in the Molecular Medicine Study Program \\ at the Georg-August University Göttingen

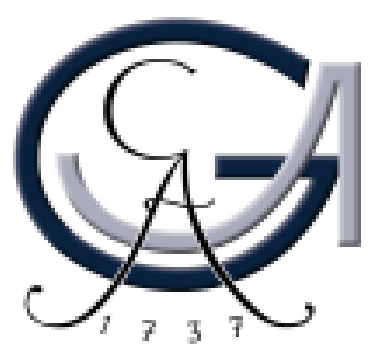

submitted by

\section{Jing Qi}

born in Jilin, P. R. China

Göttingen 2016 


\section{Members of the thesis committee:}

Supervisor:

\section{Prof. Dr. Kaomei Guan-Schmidt}

Department of Cardiology and Pneumology

University Medical Center, Georg-August University of Göttingen

Second member of the thesis committee:

\section{Prof. Dr. Dörthe Katschinski}

Institute of Cardiovascular Physiology

University Medical Center, Georg-August University of Göttingen

Third member of the thesis committee:

\section{Prof. Dr. Peter Rehling}

Department of Cellular Biochemistry

University Medical Center, Georg-August University of Göttingen

Date of Disputation: 


\begin{abstract}
AFFIDAVIT
Here I declare that my doctoral thesis entitled:

"An integrated approach using patient-specific induced pluripotent stem cells and protein biochemistry to study Vici syndrome associated cardiomyopathy"
\end{abstract}

has been written independently with no other sources and aids than quoted.

Jing Qi

Göttingen, August 2016 


\section{List of publications:}

Liu, X., J. Qi, X. Xu, M. Zeisberg, K. Guan and E. M. Zeisberg (2016). Differentiation of functional endothelial cells from human induced pluripotent stem cells: A novel, highly efficient and cost effective method. Differentiation, doi: 10.1016/j.diff.2016.05.004.

\section{Poster:}

Qi, J., M. Gautel and K. Guan. An integrated approach using patient-specific induced pluripotent stem cells and protein biochemistry to study Vici syndrome associated cardiomyopathy. European Society of Cardiology Congress 2015 in London (29 ${ }^{\text {th }}$ August 2015 to $2^{\text {nd }}$ September 2015). 


\section{Contents}

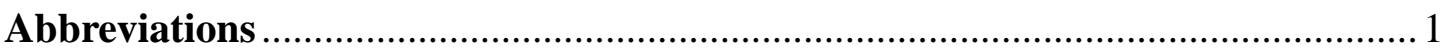

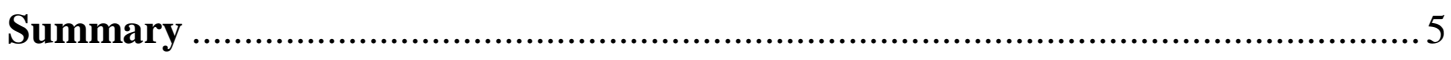

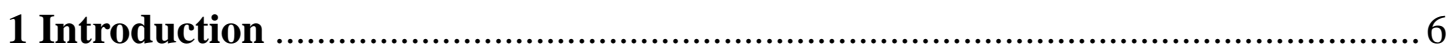

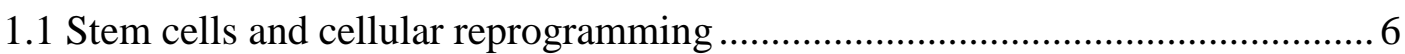

1.1.1 Definition and characteristics of stem cells .............................................. 6

1.1.2 Cellular and lineage reprogramming ....................................................... 7

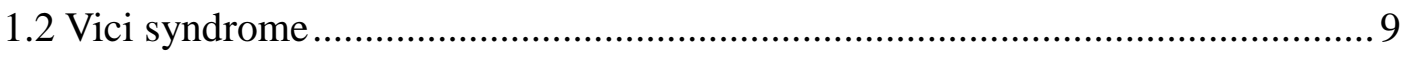

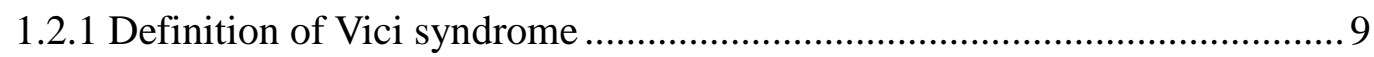

1.2.2 Myopathy and cardiomyopathy in Vici syndrome .................................. 10

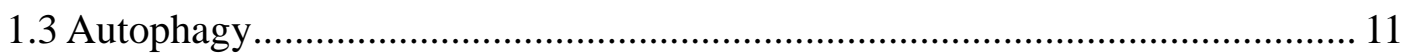

1.3.1 Autophagy defects in diseases ........................................................... 13

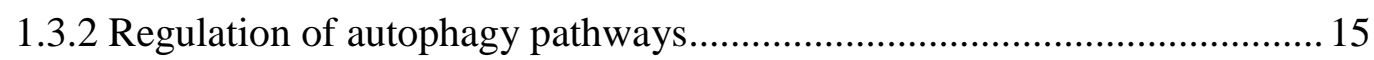

1.3.3 EPG5 functions as an autophagy regulator........................................... 17

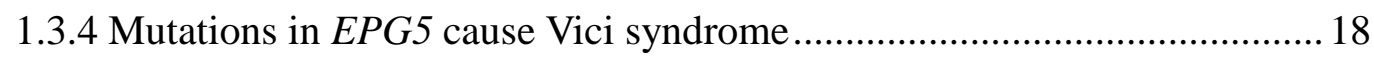

1.4 Application of iPSCs in disease modeling and drug screening ........................ 18

1.4.1 Patient-specific iPSCs used for disease modeling ................................... 18

1.4.2 Patient-specific iPSCs used for drug screening ...................................... 20

1.4.3 Genomic editing of iPSCs using the CRISPR/Cas9 technique ................. 20

1.4.4 Engineered heart muscle generated from iPSCs-derived cardiomyocytes . 21

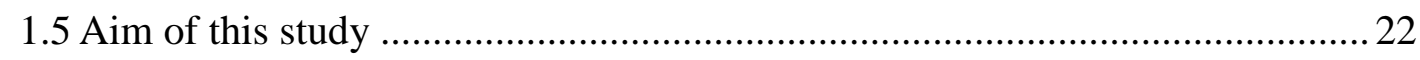

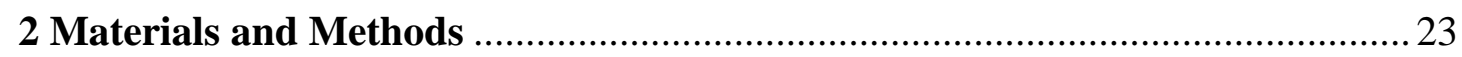

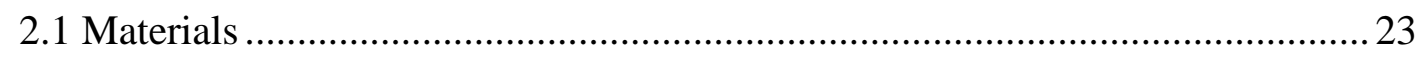

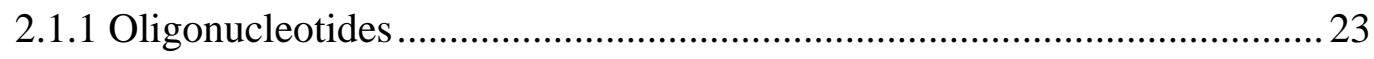

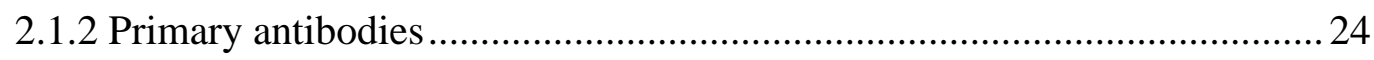

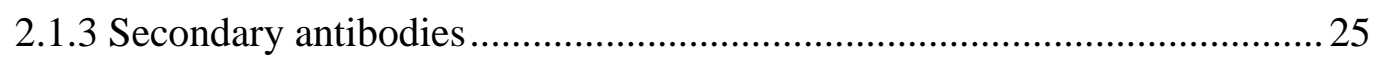

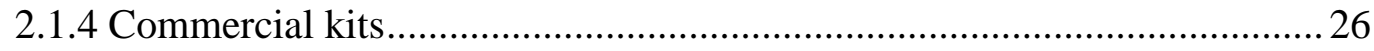

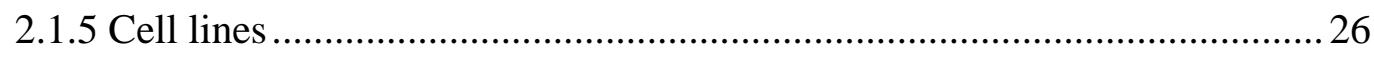

2.1.6 Basal media, growth factors, cytokines and drugs for cell culture .............26

2.1.7 Stock solutions for cell culture .......................................................... 28

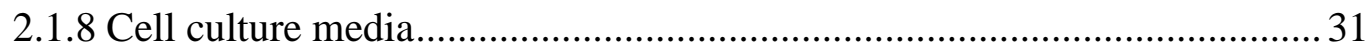


2.1.9 Chemicals and reagents for molecular biological analysis.......................32

2.1.10 Buffers and solutions for molecular biological analysis ........................ 34

2.1.11 Lab equipment and other materials ...................................................... 36

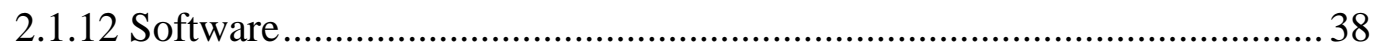

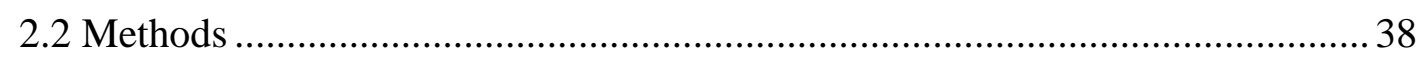

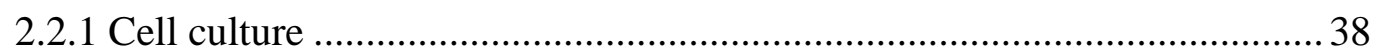

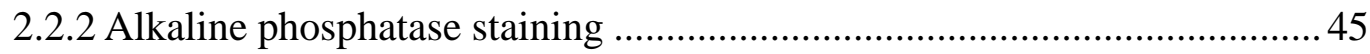

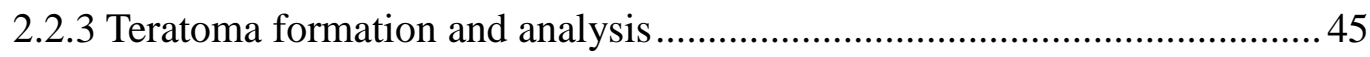

2.2.4 Isolation of genomic DNA and DNA sequencing …...............................45

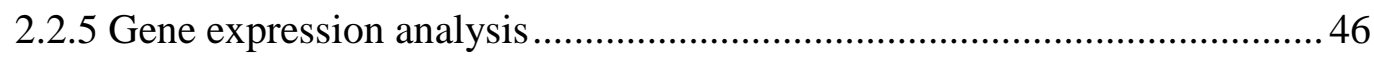

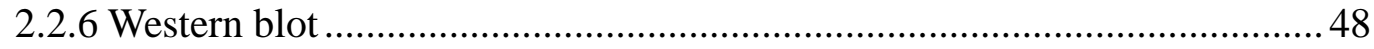

2.2.7 Immunofluorescence staining and analysis of images ........................... 49

2.2.8 Flow cytometry and cell sorting ....................................................... 51

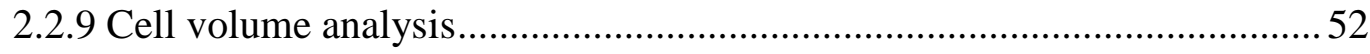

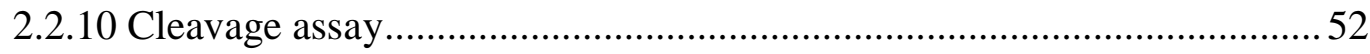

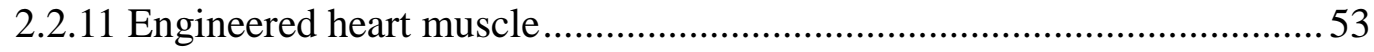

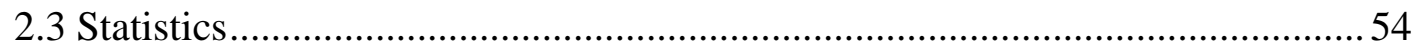

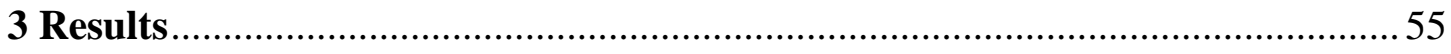

3.1 Generation of Vici-iPSCs and proof of pluripotency ....................................55

3.1.1 Generation of hiPSCs from the patient with Vici syndrome ......................55

3.1.2 Expression of pluripotent-related markers in Vici-iPSCs..........................56

3.1.3 Spontaneous differentiation of Vici-iPSCs in vitro and in vivo ..................58

3.1.4 Verification of EPG5 mutation in Vici-iPSCs ........................................5 59

3.2 Generation and phenotype analysis of Vici-iPSCs-derived

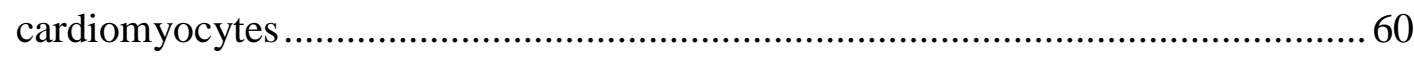

3.2.1 Differentiation of cardiomyocytes from Ctr-iPSCs and Vici-iPSCs ..........60 60

3.2.2 Vici-iPSCs-CMs show larger cell surface area but normal cell volume .... 64

3.2.3 Vici-iPSC-CMs exhibit multidirectional sarcomere organization but

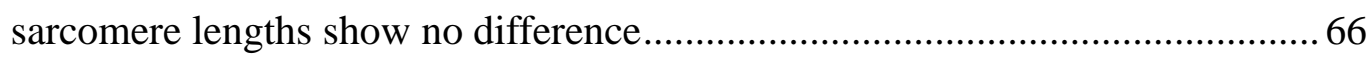

3.2.4 Functional analysis by generating engineered heart muscle ......................6 68

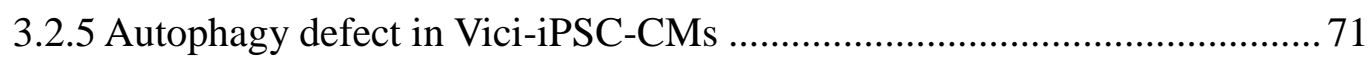

3.2.6 Abnormal AKT pathway in Vici-iPSC-CMs .......................................... 80 


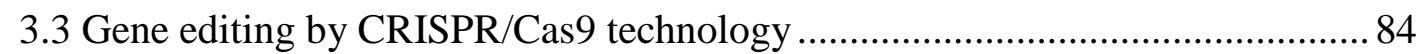

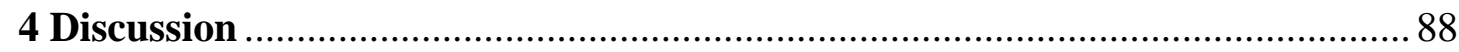

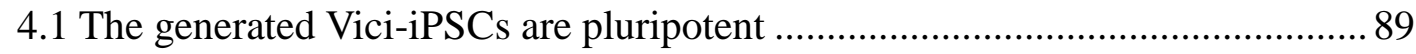

4.2 Cardiomyocytes derived from hiPSCs are embryonic-like cells ...................... 91

4.3 Vici-iPSC-CMs recapitulate the cardiac phenotype of the patient with Vici

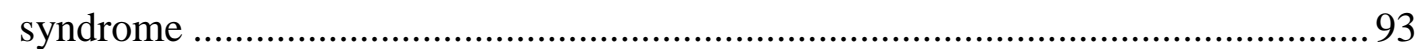

4.3.1 Vici-iPSC-CMs show disorganized sarcomere structures ........................ 93

4.3.2 The autophagic flux is blocked in Vici-iPSC-CMs ................................. 94

4.3.3 The PI3K/AKT/mTORC1 pathway is involved in the autophagy defect in

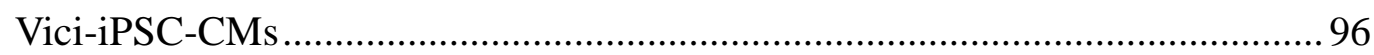

4.4 No isogenic control of Vici-iPSCs has been generated ................................... 99

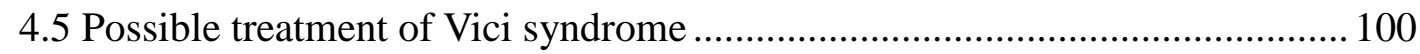

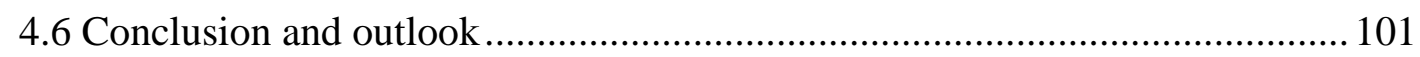

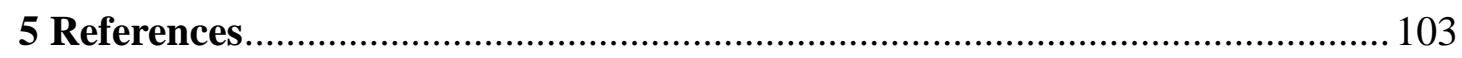

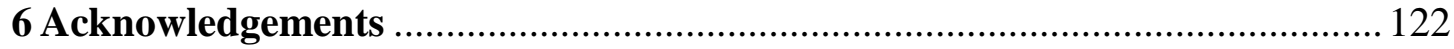

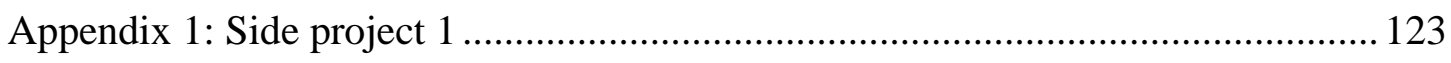

Patient-specific iPSCs modelling TTN mutation caused cardiomyopathy ............ 123

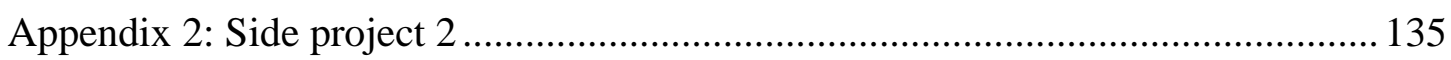

Direct differentiation of skeletal muscle cells from human induced pluripotent stem

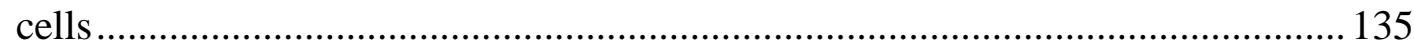

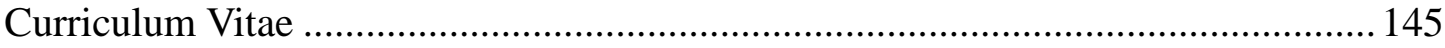




\section{Figure Lists}

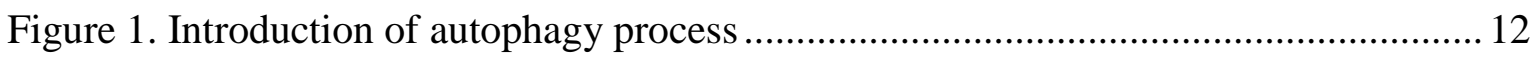

Figure 2. Schematic representation of mTOR-dependent autophagy regulation ................ 16

Figure 3. iPSCs as a platform for disease modeling and drug screening ........................... 19

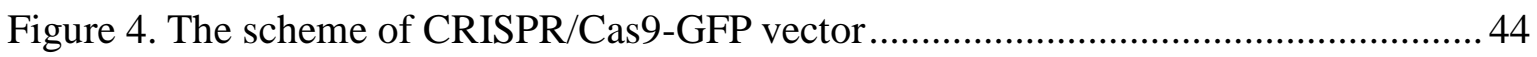

Figure 5. The scheme of pBudCE4.1-LC3-GFP-RFP vector ............................................ 44

Figure 6 . The scheme of quantitation of sarcomere structure …..................................... 51

Figure 7. Analysis of cardiomyocyte volume using flow cytometry .................................52

Figure 8 . The scheme of iPSC generation from fibroblasts derived from the patient with

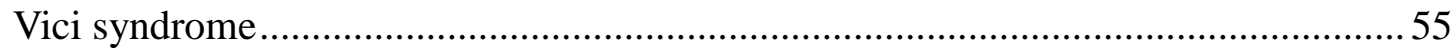

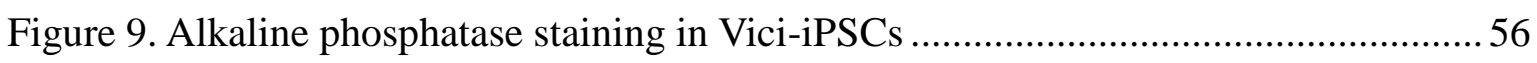

Figure 10. Expression of pluripotent-related genes in Vici-iPSCs .....................................56

Figure 11. Expression of pluripotent-related proteins in Vici-iPSCs ................................ 57

Figure 12. Spontaneous differentiation of Vici-iPSCs in vitro .......................................... 58

Figure 13. H\&E staining of teratoma generated from iVici1.3 cells in vivo ....................... 59

Figure 14. Verification of the EPG5 mutation in the genome of Vici-iPSCs .....................59

Figure 15. Verification of EPG5 mutation on mRNA level in Vici-iPSCs.......................... 60

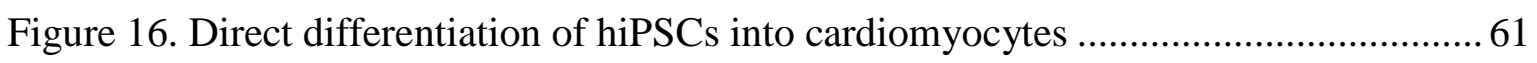

Figure 17. Efficiency of direct differentiation of hiPSCs into cardiomyocytes ...................62 62

Figure 18. Gene expression of cardiac-related markers by RT-PCR .................................. 63

Figure 19. Structural characterization of Ctr- and Vici-iPSC-CMs.................................... 63

Figure 20. Measurement of cell surface area of Ctr- and Vici-iPSC-CMs .......................... 65

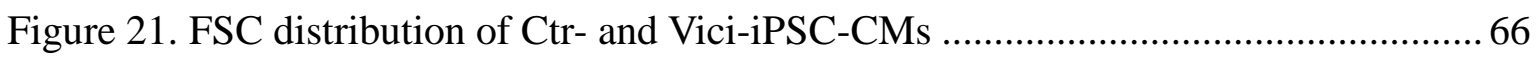

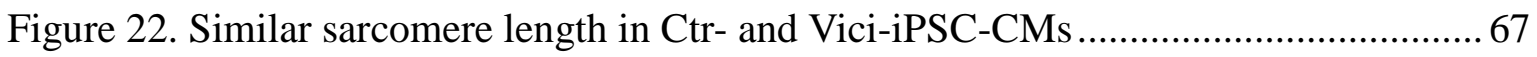

Figure 23. Quantitation of sarcomere organization of Ctr- and Vici-iPSC-CMs. ............... 68

Figure 24. Cell diameter of Ctr- and Vici-iPSC-CMs before used for generiation of EHMs

Figure 25. Measurement of contractile forces of EHMs generated from Ctr- and

Vici-iPSC-CMs. 
Figure 26. LC3I and LC3II were accumulated in Vici-iPSC-CMs .................................. 71

Figure 27. Enhanced expression of LC3 in Vici-iPSC-CMs after isoproterenol and

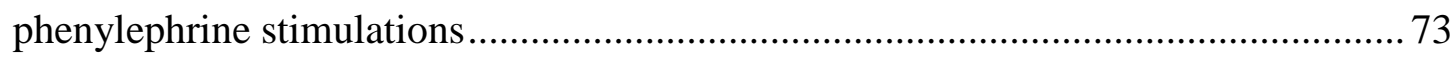

Figure 28. Accumulation of LC3II-positive puncta in Vici-iPSC-CMs in vitro.................. 74

Figure 29. Accumulation of LC3-GFP-RFP in untreated Vici-iPSC-CMs......................... 75

Figure 30. Abnormal localization of lysosomes in Vici-iPSC-CMs................................... 76

Figure 31. Blocked fusion of LC3II-positive puncta with LAMP1-positive puncta in

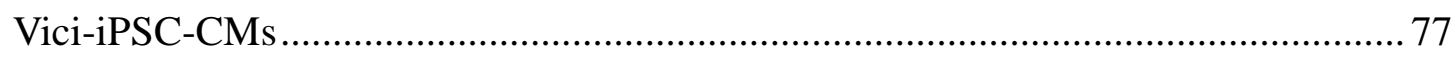

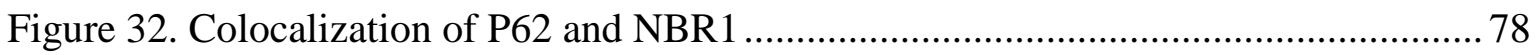

Figure 33. P62-positive puncta fused with lysosomes ................................................... 79

Figure 34. Mitochondrial distribution in Ctr- and Vici-iPSC-CMs .................................... 80

Figure 35. Reduced phosphorylation of AKT S473 and AKT T308 in Vici-iPSC-CMs ..... 81

Figure 36. Decreased phosphorylation of FOXO3 threonine 32 (T32) and FOXO1

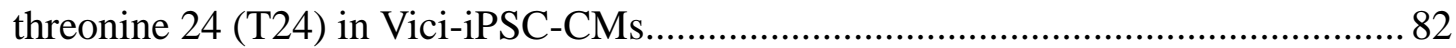

Figure 37. mTOR expression and phosphorylation in Vici-iPSC-CMs ............................ 82

Figure 38. Phosphorylation of P70S6K in Ctr-iPSC-CMs and Vici-iPSC-CMs .................. 83

Figure 39. Phosphorylation of GSK3 $\beta$ in Ctr- and Vici-iPSC-CMs .................................. 83

Figure 40. The scheme of correcting the mutation in EPG5 by using the CRISPR/Cas9

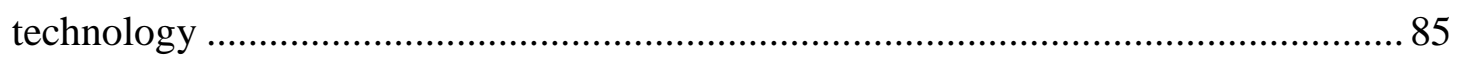

Figure 41. Assessment of cleavage efficiency of CRISPR/Cas9-GFP ............................... 86

Figure 42. Representative clones after the genomic editing ........................................... 87

Figure 43. The schematic representation of partial AKT and mTORC1 cellular pathways 97 


\section{Abbreviations}

AFP

AKT S473

AKT T308

AKT/PKB

ALS

APS

ATP

bFGF

bp

BPAN

BSA

Cas9

cDNA

CFTR

CM

CMA

COXIV

Cre

CRISPR

cTNT

Ctr

Ctr-iPSC-CMs

Ctr-iPSCs

Ctr-EHM

CX43

Cy3

DAPI

DEPC

DMEM
Alpha-1-fetoprotein

AKT serine 473

AKT threonine 308

Protein kinase B

Amyotrophie lateral sclerosis

Ammonium persulfate

Adenosine triphosphate

Basic fibroblast growth factor

Base pair

$\beta$-propeller protein-associated neurodegeneration

Bovine serum albumin

CRISPR associated endonuclease 9

Complementary DNA

Cystic fibrosis transmembrane conductor receptor

Cardiomyocyte

Chaperone-mediated autophagy

Cytochrome c oxidase subunit IV

Cyclization recombination

Clustered regularly interspaced short palindromic repeat

Cardiac troponin $\mathrm{T}$

Control

Cardiomyocytes derived from Ctr-iPSCs

iPSCs derived from healthy control

EHM generated from Ctr-iPSC-CMs

Connexin 43

Cyanine dyes

4', 6-diamidino-2-phenylindole

Diethylpyrocarbonate

Dulbecco's modified Eagle medium 


\begin{tabular}{|c|c|}
\hline DMSO & Dimethyl sulfoxide \\
\hline DPBS & Dulbecco's phosphate buffered saline \\
\hline DTT & Dithiothreitol \\
\hline E-8 & Essential 8 \\
\hline EB & Embryoid body \\
\hline EDTA & Ethylenediaminetetraacetic acid \\
\hline EHM & Engineered heart muscle \\
\hline EPG5 & Ectopic P-granules autophagy protein 5 homolog \\
\hline ER & Endoplasmic reticulum \\
\hline FBS & Fetal bovine serum \\
\hline FFT & Fast fourier transform \\
\hline FSC & Forward scatter \\
\hline FITC & Fluorescein isothiocyanate \\
\hline FOXD3 & Forkhead box D3 \\
\hline FOXO1 & Forkhead box $\mathrm{O} 1$ \\
\hline FOXO1 T24 & FOXO1 threonine 24 \\
\hline FOXO3 & Forkhead box O3 \\
\hline FOXO3 T32 & FOXO3 threonine 32 \\
\hline FWHM & Full width at half maximum \\
\hline GAPDH & Glycerinaldehyde-3-phosphate-dehydrogenase \\
\hline GD & Gaucher disease \\
\hline GDF3 & Growth differentiation factor-3 \\
\hline hESCs & Human embryonic stem cells \\
\hline hiPSCs & Human induced pluripotent stem cells \\
\hline hiPSC-CMs & Cardiomyocytes derived from hiPSCs \\
\hline HR & Homologous recombination \\
\hline HRP & Horseradish peroxidase \\
\hline IF & Immunofluorescence \\
\hline IGF1/2 & Insulin-like growth factor- $1 / 2$ \\
\hline IMDM & Iscove's modified Dulbecco's medium \\
\hline IWP2 & Inhibitor of WNT production-2 \\
\hline
\end{tabular}




$\begin{array}{ll}\text { KSR } & \text { Knockout serum replacement } \\ \text { LAMP1 } & \text { Lysosome associated protein } 1\end{array}$

LB Lysogeny broth

LC3 Microtubule-associated protein 1 light chain 3

MEFs Mouse embryonic fibroblasts

mESCs Mouse embryonic stem cells

MI Myocardial infarction

MLC2V Myosin light chain 2V

MOI Multiplicity of infection

MTG Monothioglycerol

mTOR Mammalian target of rapamycin

mTORC1 mTOR complex 1

mTORC2 mTOR complex 2

mTOR S2448 mTOR serine 2448

MYH7 Myosin heavy chain 7

MYL2 Myosin light chain 2

NANOG Nanog homeobox

NBR1 Neighbor of BRCA1 gene 1

NEAA Non-essential amino acid

NHEJ Non-homologous end joining

NKX2.5 NK2 homeobox 5

NPC Niemann-Pick disease type C

OCT4 Octamer binding transcription factor 4

P70S6K Ribosomal protein S6 kinase, $70 \mathrm{kDa}$, polypeptide 1

P70S6K T389 P70S6K threonine 389

PE Phosphatidylethanolamine

PFA Paraformaldehyde

PI3K Phosphatidylinositol 3-kinase

PIP2 Phosphatidylinositol (4,5)-bisphosphate

PIP3 Phosphatidylinositol (3,4,5)-trisphosphate

PSF Pro survival factor 
RHEB Ras homolog enriched in brain

RPMI Roswell Park Memorial Institute

RT-PCR Reverse transcription PCR

SDS-PAGE Sodium dodecyl sulfate polyacrylamide gel electrophoresis

SgRNA Specific guide RNA

SMA Smooth muscle actin

SOX2 SRY (Sex determining region Y)-box 2

SSEA4 Stage-specific embryonic antigen-4

TAC Transverse aortic constriction

TB buffer Tris-borate buffer

TBST Tris-buffered saline with Tween 20

TEMED Tetramethylethylenediamine

TFEB Transcription factor EB

TNNT2 Troponin T type 2

Tris Tris (hydroxymethyl) aminomethane

TSC1/2 Tuberous sclerosis complex $1 / 2$

TZV Thiazovivin

UBD Ubiquitin-binding domain

UPS Ubiquitin-proteasome system

Vici-iPSC-CMs Cardiomyocytes derived from Vici-iPSCs

Vici-iPSCs iPSCs derived from patient with Vici syndrome

Vici-EHM EHM generated from Vici-iPSC-CMs

WB Western blot 


\section{Summary}

Vici syndrome is a rare autosomal recessive inherited multisystem disorder caused by mutations in EPG5. More than $80 \%$ of the patients with Vici syndrome suffer from cardiomyopathy. In this study, the induced pluripotent stem cell (iPSC) system was applied to model the disease, investigate the cardiac phenotype and to elucidate the underlying molecular mechanism of autophagy in cardiomyocytes derived from iPSCs. HiPSCs from the patient with Vici syndrome (Vici-iPSCs) carrying a homologous intronic mutation of EPG5 (c.4952+1G>A) were generated. These Vici-iPSC lines were pluripotent and could be differentiated into functional cardiomyocytes. Analysis of sarcomeric structure of cardiomyocytes derived from Vici-iPSCs (Vici-iPSC-CMs) showed they possessed a larger cell surface area with a higher inhomogeneity of sarcomere length compared with the cardiomyocytes derived from the Ctr-iPSCs (Ctr-iPSC-CMs). Furthermore, analysis of autophagy-related marker LC3 and lysosome associated protein 1 (LAMP1) demonstrated numerous accumulation of autophagosomes, abnormal perinuclear localization of lysosomes as well as the fusion blockade of autophagosomes with lysosomes in Vici-iPSC-CMs. Further investigation of the regulatory pathway of autophagy showed that the level of AKT phosphorylation at two activating sites (serine 473 and threonine 308) was decreased in Vici-iPSC-CMs compared to Ctr-iPSC-CMs. In addition, to figure out if these phenotypes in Vici-iPSC-CMs are caused by the EPG5 mutation, the genomic editing tool CRISPR/Cas9 was used to rescue the mutation site in EPG5. After transduction of CRISPR/Cas9-GFP and single-stranded oligonucleotides, genotype analysis of the 150 surviving Vici-iPSC colonies illustrated that non-homologous end joining (NHEJ) happens to repair the DNA double-strand break in two iPSC colonies. However, the mutation site in EPG5 was not rectified in any Vici-iPSC colonies. The data of this study illustrate that patient-specific iPSCs can be used to model Vici syndrome associated cardiomyopathy and provide a good platform to understand the function of autophagy in cardiomyocytes. 


\section{Introduction}

\subsection{Stem cells and cellular reprogramming}

\subsubsection{Definition and characteristics of stem cells}

Stem cells are undifferentiated cells, which possess the capacity to differentiate into specialized cells and to self-renew. Depending on their origin, stem cells are divided into two types: embryonic stem cells (ESCs) and adult stem cells. ESCs derived from the inner cell mass of an early blastocyst are pluripotent and have the capacity to develop into all derivatives of three embryonic germ layers and germ cells. Adult stem cells originated in different tissues are multipotent or unipotent and only capable of generating its committed cell types.

The self-renewal and differentiation potential of stem cells make them useful for studying embryogenesis and genetic diseases. Martin Evans firstly isolated mouse embryonic stem cells (mESCs) in 1981 and subsequent studies further established that leukemia inhibitor factor was essential for the long-term cultures of mESCs to maintain them in an undifferentiated state (Evans and Kaufman, 1981; Smith et al., 1988). The difficulty to obtain the human blastocyst impedes the isolation of human embryonic stem cells (hESCs) in vitro. HESCs were first isolated in 1994 (Bongso et al., 1994) and subsequently, cell lines were established when the cells were cultured on inactivated mouse embryonic fibroblasts (MEFs) as a feeder layer in medium containing basic fibroblast growth factors (bFGFs) (Bongso et al., 1994; Thomson et al., 1998). The traditional feeder layer culture of hESCs is time-consuming due to the necessity of inactivated MEFs as well as the bias towards differentiated cells in long-term cultures. To avoid this, advancement in cell attachment and cell culture conditions for hESCs with chemical defined medium were pursued, for example, mTeSR and Essential 8 (E-8) medium combined with a gelatinous protein mixture such as Matrigel and Geltrex (Chen et al., 2011; Vallier, 2011). These improvements, feeder- and serum-free culture conditions, lead to controllable and reproducible conditions for growth and amplification 
of hESCs, which make the use of hESCs feasible for studying embryogenesis and modeling disease.

Furthermore, the unique properties of self-renewal and differentiation possessed by hESCs have great prospects for regenerative medicine. However, the supply and the destruction of blastocysts for the generation of hESCs are medically and ethically controversial, thus limit the use of these cells in disease treatments (Solter, 2006). The breakthrough in the discovery of human induced pluripotent stem cells (hiPSCs) by Shinya Yamanaka (Takahashi et al., 2007), which were generated from human fibroblasts derived from skin biopsy, circumvented the medical and ethical dilemma associated with hESCs. As a result, hiPSCs become widely available to research groups, allowing these cells to be exploited for disease modeling, drug discovery and potential cell replacement therapeutics.

\subsubsection{Cellular and lineage reprogramming}

Embryogenesis is a naturally and uni-directionally occurring process where a fertilized egg develops into specialized somatic or germ cells. Upon exposure to growth factors and resulting signaling cues, the cells become committed in their fates and are believed to be irreversible. However, the concept of reversing cell fate is verified by cellular reprogramming and lineage reprogramming, which includes the conversion of differentiated somatic cells back to an embryonic state and the transdifferentiation of one specialized cell type into another.

Cell reprogramming was first demonstrated by using somatic cell nuclear transfer technology in frogs where the nucleus from a differentiated cell was injected into an unfertilized and enucleated egg resulting in viable tadpoles (Gurdon, 1962). Three decades later, Ian Wilmut cloned "Dolly" the sheep and demonstrated for the first time that mammalian epithelial cells could be reverted back to pluripotent cells through cellular reprogramming (Wilmut et al., 1997). Later, other mammalian animals had been successfully cloned (Hochedlinger and Jaenisch, 2002; Wakayama and Yanagimachi, 
1999). Inducing pluripotency was further confirmed by fusing adult thymocytes with ESCs (Tada et al., 2001). These studies suggest that a set of unknown factors in oocytes and ESCs are responsible for the interplay of pluripotency and somatic state in cells.

In 2006, these factors that allowed nuclear reprogramming were discovered. The research group led by Prof. Yamanaka successfully reprogrammed mouse somatic cells into the pluripotent state by using the retroviral delivery of four key genes; oct3/4 (octamer binding transcription factor 3/4), sox2 (SRY-box 2), klf4 (Krüppel-like factor 4), and c-myc (Takahashi and Yamanaka, 2006). They initially screened 24 candidates that were highly expressed in ESCs for pluripotency and made a series of different combinations to transduce MEFs. Finally, the master transcription factors oct3/4, sox2, klf4 and c-myc were deemed to fulfill the induction of pluripotency from somatic cells. These reprogrammed cells were termed induced pluripotent stem cells (iPSCs) and demonstrated similar features of ESCs including morphology, growth properties and expression of ESC markers.

The similar approach was subsequently used to generate iPSCs from human, monkey, pig, dog and rat (Esteban et al., 2009; Honda et al., 2010; Li et al., 2009; Liao et al., 2009; Liu et al., 2008; Shimada et al., 2010; Takahashi et al., 2007; Yu et al., 2007). Additionally, the different types of somatic cells were successfully reprogrammed into iPSCs and fewer transcription factors were subsequently found to sufficiently induce pluripotency in some cell types, such as adult neural stem cells, in which OCT4 alone was sufficient to achieve pluripotency (Kim et al., 2009b). The iPSCs avoid the ethical concerns associated with hESCs and provide researchers potentially the unlimited supply of pluripotent stem cells for studying molecular mechanisms of diseases and their application in regenerative medicine.

However, retroviral-mediated transduction may cause random integration of transgenes and potential oncogenes into the host cell genome, which could lead to undesirable effects such as tumor formation. Further relatively "safer" approaches of generating 
integration-free iPSCs were developed. These approaches allowed the delivery of the genes using other platforms such as plasmid transfection, adenovirus, Sendai virus, proteins, RNAs and small molecules (Fusaki et al., 2009; Hou et al., 2013; Kim et al., 2009a; Kim et al., 2011; Loh et al., 2009; Okita et al., 2011; Okita et al., 2008; Stadtfeld et al., 2008; Warren et al., 2010). The generation of iPSCs is a process with a low efficiency, typically less than $1 \%$ of transfected or transduced cells become iPSCs. Pluripotency induction with combined proteins was proved to be more challenging with even lower efficiency. The plasmid transfection and Sendai virus transduction are now routinely used in many laboratories due to their simplicity and reproducibility (Yamanaka, 2012).

Furthermore, transdifferentiation (lineage reprogramming) where one specialized cell type directly converts into another was accomplished through ectopic expression of the master transcription factors specific for the cell lineage. In 1987, Davis reported that MEFs were able to be converted into myoblasts through myod overexpression (Davis et al., 1987). Recently, fibroblasts were successfully reprogrammed into functional cardiomyocytes from different species by overexpression of a set of cardiac-specific key factors, such as GATA4, MEF2C, and TBX5 (Cao et al., 2016; Ieda et al., 2010; Jayawardena et al., 2012; Qian et al., 2012; Song et al., 2012). Direct conversion of mouse fibroblasts to functional neurons was fulfilled by the defined factors and small molecules, such as brn2 (also known as pou3f2), ascl1 and myt1l (Pang et al., 2011; Vierbuchen et al., 2010; Xue et al., 2013).

\subsection{Vici syndrome}

\subsubsection{Definition of Vici syndrome}

Vici syndrome is a multisystem disorder characterized by five key pathological features: callosal agenesis, cataracts, cardiomyopathy, hypopigmentation and combined immunodeficiency (Dionisi Vici et al., 1988). Apart from these five principal diagnostic findings, a wide range of variably present additional features has been reported (Cullup et al., 2013; Byrne et al., 2016a). Vici syndrome is a rare autosomal recessive inherited 
disease caused by mutations in EPG5 (ectopic P-granules autophagy protein 5) that locates on chromosomal 18q12.3 (Cullup et al., 2013). Until now, there have been at least 50 cases of Vici syndrome reported worldwide. Besides the classical clinical features reported, renal tubular acidosis, neuromuscular involvement, sensorineural hearing loss and sleep abnormalities can develop into other clinical complications in patients suffering from this syndrome (Al-Owain et al., 2010; Chiyonobu et al., 2002; del Campo et al., 1999; Ehmke et al., 2014; El-Kersh et al., 2015; Filloux et al., 2014; Finocchi et al., 2012; Huenerberg et al., 2016; Lu et al., 2016; McClelland et al., 2010; Miyata et al., 2014; Miyata et al., 2007; Ozkale et al., 2012; Rogers et al., 2011; Said et al., 2012; Tasdemir et al., 2016).

The frequency of clinical features was evaluated depending on the 38 genetically confirmed cases (Byrne et al., 2016a). All of these patients exhibit an absence of corpus callosum, gross development delay and immunological deficiencies. 97\% of the patients are with median survival around 42 months (range 1 to 102 months). Additionally, most patients exhibit a pale complexion and light hair; $90 \%$ of the patients acquire microcephaly; $82 \%$ of the patients suffer from cardiomyopathy; $76 \%$ develop cataracts. Furthermore, additional dysfunctions of other organs including thymus, lungs, thyroid, liver, kidney and blood in most patients are also observed. Hence, despite Vici syndrome being a rare disease, its existence can result in multiple disorders and the mechanism of disease progression remains to be elucidated.

\subsubsection{Myopathy and cardiomyopathy in Vici syndrome}

Patients with Vici syndrome exhibit defective skeletal muscle and cardiomyocytes. The marked hypotonia, weak movement and delayed motor neuron development in patients with Vici syndrome reflect skeletal myopathy. Studies using skeletal muscle biopsy from the patients revealed variable myofibril size, centralized nuclei, hypotrophic fibers and accumulated glycogen (Al-Owain et al., 2010; McClelland et al., 2010). Disorganized sarcomeres, abnormal localization of mitochondria (ring-shape) and large autophagy vesicles were also observed. 
Notably, majority (around 80\%) of affected children exhibited hypertrophic or dilated cardiomyopathy especially in the left ventricle. In addition, $10 \%$ of patients were reported to have minor congenital heart defects including open patent foramen ovale, ventricular or atrial septal defects, hypoplastic aortic arch or mitral valve insufficiency (Byrne et al., 2016a). Histo-pathological analysis of the heart tissue revealed that vacuoles and membrane bound cytoplasmic inclusions existed in cardiomyocytes (Miyata et al., 2014). The cardiomyocytes also showed increased staining for autophagy markers LC3 (microtubule-associated protein 1 light chain 3) and P62 on immunohistochemistry. While cardiomyopathy is usually detected in early life of patients with Vici syndrome, intermittent deterioration of cardiac function can occur during the progression of the disease in the later stages further complicated by failure of respiratory system and heart function. The cardiomyopathy in patients with Vici syndrome suggests that autophagy has an important function in the human heart, which requires further investigation using appropriate models.

\subsection{Autophagy}

Autophagy plays a key role in removing damaged proteins and deteriorated organelles and in supplying cellular energy and nutrients (Kaur and Debnath, 2015). Autophagy is a conserved catabolic process with the capacity to degrade long-lived proteins and organelles such as aggregated proteins, mitochondria, ribosomes and peroxisomes. In contrast to autophagy, the ubiquitin-proteasome system (UPS) is the other key mechanism of maintaining the protein hemostasis, which targets the short-lived proteins (Hershko and Ciechanover, 1998; Shaid et al., 2013).

Autophagy can be divided into three types, including the macroautophagy, microautophagy and chaperone-mediated autophagy (CMA). Macroautophagy, generically known as autophagy, is the main pathway involving the formation of autophagosomes and autolysosomes to degrade the proteins. Microautophagy is the process where cytoplasmic degraded components are directly engulfed by lysosomes with the process of invagination of the lysosomal or endosomal membrane (Li et al., 2012). 
CMA differs from macroautophagy and microautophagy because degraded proteins are translocated without membrane invagination. Proteins that contain a KFERQ (lysine-phenylalanine-glutamic acid-arginine-glutamine) motif are recognized by the chaperone heat shock cognate $70 \mathrm{kDa}$ protein (HSC70), which could bind to lysosome associated membrane protein 2A (LAMP2A). By this way, the substrate proteins cross the lysosomal membrane for subsequent degradation (Cuervo and Wong, 2014) (Figure 1A).

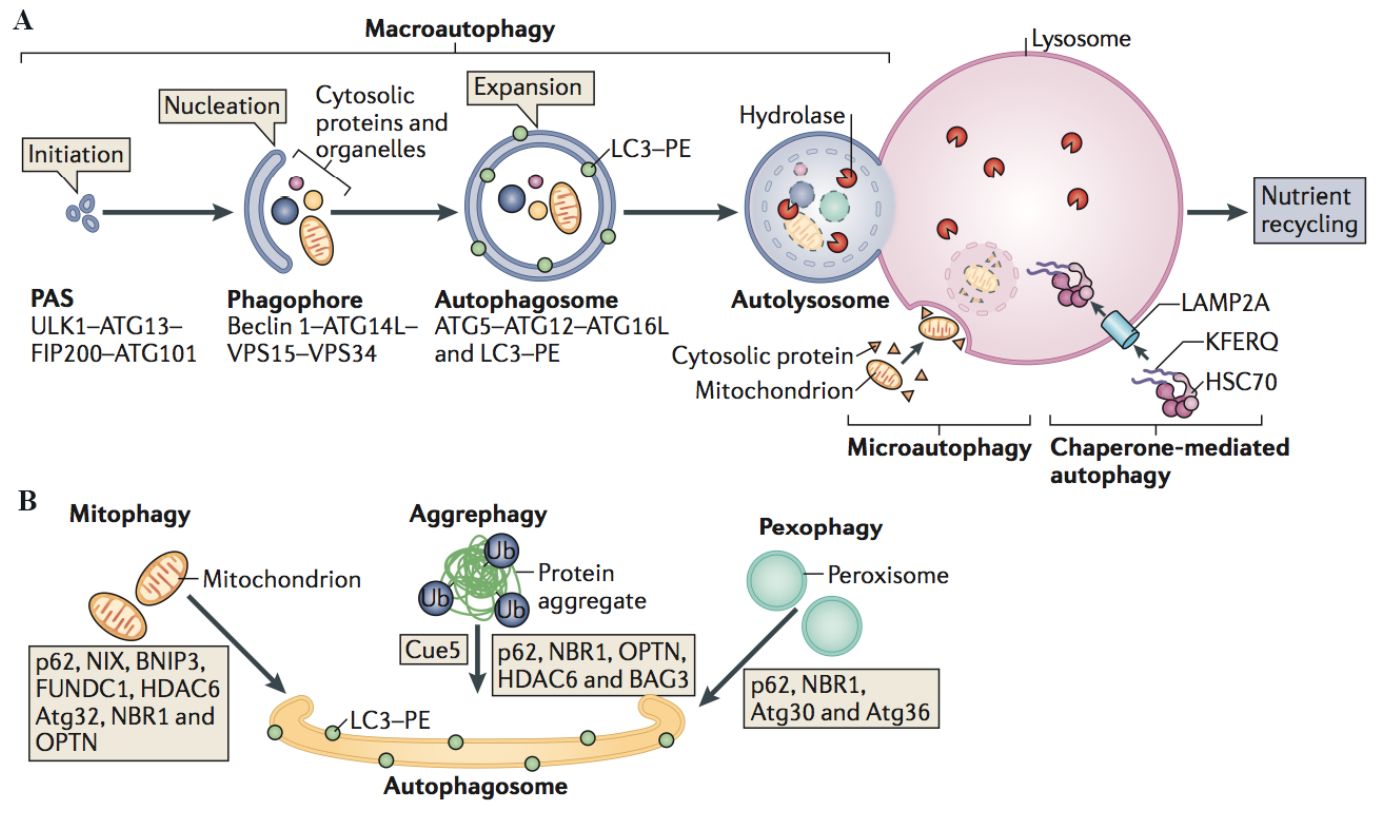

Figure 1. Introduction of autophagy process. A, Autophagy is classified into three types. Macroautophagy is a stepwise process that involves the following: 1) initiation and isolation of membrane, phagophores; 2) nucleation of phagophores; 3) elongation and maturation of phagophores into autophagosomes; 4) autophagosomes fusing with lysosomes, called autolysosomes; 5) degradation in the lysosomes. This intricate process is regulated by different complex. The phagophores assembly site (PAS) is mediated by the UNC51-like kinase (ULK) complex, which contains ULK1, autophagy related protein 13 (ATG13), FAK family kinase interacting protein of $200 \mathrm{kDa}$ (FIP200) and ATG101. The nucleation of phagophores is regulated by the class III PI3K complex, which consists of beclin1, ATG14L, vacuolar protein sorting 15 (VPS15) and VPS34. The elongation and maturation of phagophores is controlled by a complex, which is composed of ATG5, ATG12, ATG16L and PE-LC3. Chaperone mediated autophagy (CMA) requires heat shock cognate $70 \mathrm{kDa}$ protein (HSC70) that can recognize the KFERQ-motif-containing degraded proteins, which are translocated into lysosomes with binding of HSC70 to lysosome associated membrane protein 2A (LAMP2A). Microautophagy engulfs the proteins directly with the invagination of lysosomal or endosomal membranes. B, The selective autophagy of degraded organelles such as mitochondria, pathogens, ribosomes, peroxisomes, endoplasmic reticulum and aggregated proteins is mediated by autophagy adaptors including P62 and NBR1 through their UBD and LIR domain. The figure was taken from (Kaur and Debnath, 2015). 
Autophagy was initially thought to be a process attributed to nutrient starvation (induced autophagy), which represents non-selective degradation of cytoplasmic components (De Duve et al., 1955; De Duve and Wattiaux, 1966). However, this was later observed to be a selective process, in which the partially degraded aggregated proteins and organelles are selectively sequestered into autophagosomes (Bjorkoy et al., 2005; Kirkin et al., 2009a). Based on the substrate, selective autophagy was separated into mitophagy (mitochondria), xenophagy (pathogens), ribophagy (ribosomes), pexophagy (peroxisomes) and reticulophagy (endoplamatic) (Shaid et al., 2013). The recognition of selective degraded targets involves the ubiquitin binding proteins, such as P62/SQSTM1 and NBR1 (next to BRCA1 gene 1). P62 and NBR1 are autophagy adaptors, containing the ubiquitin-binding domain (UBD) and LC3-interacting region (LIR), which form a link between the degraded components and the autophagosomes (Kirkin et al., 2009b) (Figure 1B). LC3 is the most widely used autophagic marker that goes through a complex translational modifications. After synthesis of LC3, LC3I that located in the cytosol is immediately generated by cleaving the carboxyl terminal region of LC3 while conjugating to phosphatidylethanolamine (PE) of LC3I results in LC3II, which is located in the autophagosomal membrane. The conversion between LC3II and LC3I suggests the autophagic activity. With the function of LC3, P62 and NBR1, they act as autophagy markers to indicate dynamics of autophagy.

\subsubsection{Autophagy defects in diseases}

Autophagy can prevent the proteins and organelles from accumulating, on the other hand, the metabolites derived from proteins, lipids, ferritin and glycogen are recycled for diverse anabolisms including the protein, lipid and glycogen synthesis (Kaur and Debnath, 2015). The importance of autophagy was confirmed by knocking out essential autophagy related genes, such as atg4, atg5, atg6 and atg7 (Kuma et al., 2004; Marino et al., 2007; Pua et al., 2007; Qu et al., 2007; Tanaka et al., 2000). Autophagy-defective oocytes derived from the oocyte-specific atg 5 knockout mouse failed to develop beyond the fourand eight-cell stages when they were fertilized with atg5-null sperm (Tsukamoto et al., 2008). The liver-specific atg7 knockout mouse showed protein aggregates, aberrant 
mitochondrial structure and reduced removal of peroxisomes (Iwata et al., 2006). The atg7-null mouse was only able to survive 1 day after birth due to the lack of nutrients and energy (Komatsu et al., 2005).

Autophagy dysfunction was also observed in the biopsies of muscles, liver, immune system and other organs, which were derived from various human diseases such as cancer and neurodegeneration diseases (Ezaki et al., 2011; Karantza-Wadsworth et al., 2007; Levine and Kroemer, 2008; Tannous et al., 2008). Autophagy deficiency in Alzheimer disease was shown by the accumulation of autophagic vacuoles in the affected neurons and reduced BECLIN1 expression in the brain tissue, which regulates the autophagosome formation (Nixon et al., 2005; Pickford et al., 2008; Small et al., 2005). In addition, the missense point mutation was identified in autophagy receptor gene P62/SQSTM1 in some patients with amyotrophie lateral sclerosis (ALS) (Hirano et al., 2013; Teyssou et al., 2013). The $\beta$-propeller protein-associated neurodegeneration (BPAN) led to the reduced autophagic activity because of mutations in WDR45/WIPI14, a core autophagy gene (Haack et al., 2012; Saitsu et al., 2013). Autophagy is also involved in the tumorigenesis, in which autophagy as a candidate of tumor suppressor was discovered in 1999 (Liang et al., 1999). Decreased expression of autophagy gene BECLIN1 in human breast, ovarian and brain tumors and inhibited growth of cancer cells by ectopic expression of BECLINI in tumor cell lines in vitro illustrated the important role of autophagy in suppressing tumor generation (Liang et al., 1999).

Autophagy defects such as failure of autophagosomes fusing with lysosomes or the aggregation of proteins that exceed the capacity of autophagosome clearance has been linked to the pathogenesis of muscle diseases (Levine and Kroemer, 2008). Autophagosomes are abnormally accumulated in cardiac biopsies from patients with coronary artery disease, hypertension, aortic valvular disease and congestive heart failure (Terman and Brunk, 2005). As cardiac remodeling is a chronic maladaptive process, characterized by progressive ventricular dilatation, cardiac hypertrophy, fibrosis, and deterioration of cardiac performance (Nishida and Otsu, 2015), the dynamic autophagy in 
human heart is hard to observe due to the difficulty in obtaining human heart tissue. To elucidate the function of autophagy in the heart, mouse models are widely used to study the events involving the heart disease, which replicate specific disease situations of myocardial infarction (MI) and heart failure by ligation of a coronary artery or transverse aortic constriction (TAC). Evidence of relationship between autophagy and cardiomyopathy was demonstrated by specifically removing atg5 in cardiomyocytes in the adult mouse with phenotypic severe heart failure, observed by ventricular dilation, contractility dysfunction, irregular sarcomeres, aberrant mitochondrial structure and abnormal calcium transient after enhanced susceptibility to acute pressure overload (Kitsis et al., 2007; Nakai et al., 2007). In the pressure overload mouse model, autophagy activity was reduced following TAC induced hypertrophy, but increased during heart failure (Nishida et al., 2009). In the MI mouse model, autophagy was activated during the sub-acute and chronic stages after coronary occlusion (Kanamori et al., 2011). The dynamic autophagy during cardiac remodeling in the mouse models suggests that autophagy is protective to remove the excessive toxic proteins and damaged organelles but not sufficient against the deteriorate progression.

\subsubsection{Regulation of autophagy pathways}

Autophagy plays a key role in cell homeostasis for removing the damaged proteins and organelles, which is governed by multiple signal pathways. The mammalian target of rapamycin (mTOR), which regulates cell growth, proliferation, differentiation and survival, tumor cell motility, gene transcription and protein synthesis, is the most important protein for the autophagy control (Neufeld, 2010). The mTOR pathway involves the mTOR complex 1 (mTORC1) and the mTOR complex 2 (mTORC2). The phosphoinositide 3-kinase (PI3K) pathway is the major signaling cascade controlling mTOC1 and regulating autophagy (Figure 2). Briefly, growth factors such as insulin-like growth factor-1/2 (IGF1/2) and insulin bind to insulin receptors (IR) and activate PI3K via insulin receptor substrate (IRS). Activated PI3K converts PIP2 (phosphatidylinositol (4,5)-bisphosphate) to PIP3 (phosphatidylinositol (3,4,5)-trisphosphate), which recruits PDK1 and AKT to the plasma membrane and AKT is phosphorylated and activated, 
which in turn phosphorylates and inactivates tuberous sclerosis complex (TSC) 1/2. The upstream regulator of mTORC1 is RHEB (Ras homolog enriched in brain), which directly activates mTORC1 expression. RHEB is inhibited by TSC1/2 (Boya et al., 2013), whose activity is negatively regulated by AKT.

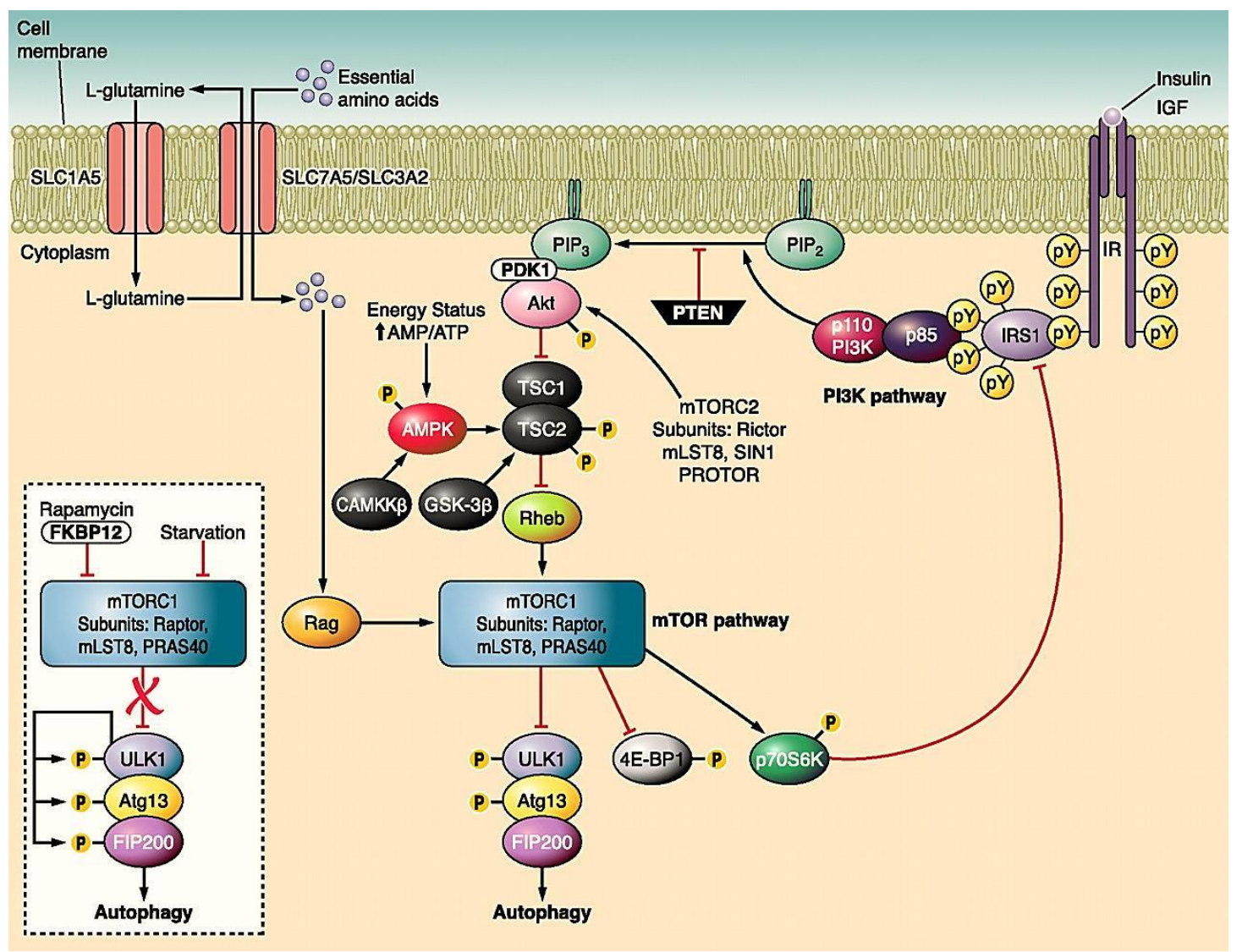

Figure 2. Schematic representation of mTOR-dependent autophagy regulation. Growth factors or insulin bind to insulin receptors (IR) and activate PI3K (phosphatidylinositol 3-kinase) via insulin receptor substrate (IRS). PI3K converts PIP2 to PIP3, which recruits PDK1 and AKT to the plasma membrane and AKT is phosphorylated and activated, which in turn phosphorylates and inactivates TSC1/2 complex. Inactivated TSC1/2 leads to activation of RHEB and consequently mTORC1 for autophagy inhibition. AMPK and GSK3 $\beta$ phosphorylate TSC1/2 complex and result in mTORC1 inactivation to induce autophagy. Amino acids such as glutamine activate mTORC1 via Rag GTPase, which inhibits ULK1-Atg13-FIP200 complex and suppress autophagy. The mTORC1 consists of mTOR, the regulatory-associated protein of mTOR (Raptor) and mammalian lethal with SEC13 protein 8 (MLCST8), is rapamycin sensitive and regulates gene transcription, protein translation and autophagy. The mTORC2 consists of mTOR, rapamycin-insensitive companion of mTOR (Rictor) and MLCST8, is rapamycin insensitive and regulates the AKT phosphorylation. PIP2: Phosphatidylinositol (4,5)-bisphosphate; PIP3: Phosphatidylinositol (3,4,5)-trisphosphate. The figure was taken from (Ravikumar et al., 2010).

Besides the PI3K/AKT/mTORC1 pathway, other signaling pathways can also regulate autophagy such as AMP-activated protein kinase (AMPK), glycogen synthase kinase 3 
beta (GSK3 $\beta$ ) and amino acid. Inositol-1,4,5-trisphosphate (IP3) and its receptor (IP3R) control the calcium translocation from endoplasmic reticulum (ER) to mitochondria where enough ATPs are generated to activate AMPK. AMPK induces autophagy through phosphorylation of TSC2 to suppress mTORC1 expression (Cardenas et al., 2010; Criollo et al., 2007; Sarkar and Rubinsztein, 2006). GSK3 $\beta$ can also phosphorylate TSC2 to induce autophagy while the essential amino acid suppresses autophagy by activating mTORC1. Rapamycin as an inhibitor of mTORC1 can be used to induce autophagy.

Apart from the mTOR pathway, there are some other mTOR-independent proteins that are also involved in the autophagy regulation. For instance, second messenger cAMP negatively regulates autophagy through the inhibition of ATG1, ATG8 and ATG13 (Graef and Nunnari, 2011). The human tumor suppressor P53 possesses a dual role on regulating autophagy. Cytoplasmic P53 suppresses autophagy while nuclear P53 induces autophagy in response to the cell trauma such as DNA damage (Maiuri et al., 2010; Ryan, 2011). Chromatin remodeling enzymes, such as histone acetyltransferases HDAC6 and SIRTUIN-1 could negatively modulate autophagy (Simms-Waldrip et al., 2008). Collectively, the complex regulation of signaling pathways involved in autophagy allows cells to maintain catabolism and metabolism.

\subsubsection{EPG5 functions as an autophagy regulator}

Initially, EPG5 was discovered as a key regulator of the autophagy pathway in C. elegans for autolysosome formation to degrade metabolic waste (Tian et al., 2010). Knockdown of epg 5 with siRNA in C. elegans caused severe accumulation of autophagosomes that were demonstrated by the lc3ii positive puncta. Additionally, in the intestinal cells of epg5 mutant C. elegans, abnormal hybrid of late endosomes and lysosomes indicated a new role of epg5 in lysosome biogenesis. The blockade of fusion of autophagosomes with lysosomes was observed in EPG5-mutant fibroblasts from patients with Vici syndrome (Cullup et al., 2013). Furthermore, the importance of epg5 was further confirmed in the epg5-knockout mice, which demonstrated phenotypes similar to that of ALS disease including muscle denervation, myofiber atrophy, late-onset progressive 
hindquarter paralysis and dramatically reduced survival. The phenotype of epg5-knockout mice recapitulates the autophagy defects and skeletal muscle myopathy seen in patients with Vici syndrome (Zhao et al., 2013). Recent studies with conditional epg5-knockout drosophila revealed a loss of function of retinal photoneurons and the autophagy defect (Byrne et al., 2016b). Taken together, EPG5 is essential for maintaining basal autophagy to keep the cell in homeostasis.

\subsubsection{Mutations in EPG5 cause Vici syndrome}

In 2013, Thomas Cullup and his colleagues discovered that mutations in EPG5 (previously KIAA1632) caused Vici syndrome. They performed exome and Sanger sequencing analysis in 18 patients with Vici syndrome, which included 11 European, 3 Arab, 2 Turkish, 1 Japanese and 1 British-Asian origin (Cullup et al., 2013). In 2016, additional 20 affected individuals were recruited in the study. They identified a total of 39 different EPG5 mutations; 55\% homozygous and 45\% compound heterozygous mutations (Byrne et al., 2016b). From the family history, they found $43 \%$ parental consanguinity, corresponding to the efficiency of the homozygous mutation. The mutation of EPG5 in patients proves to be a valuable marker and utility gene card for diagnosing Vici syndrome (Cullup et al., 2014).

\subsection{Application of iPSCs in disease modeling and drug screening}

\subsubsection{Patient-specific iPSCs used for disease modeling}

To elucidate the molecular mechanism of genetic disorders, hiPSCs are used to model the disease in a culture system in vitro. HiPSCs are able to differentiate into different cell types such as neurons, hepatocytes, smooth muscle cells, cardiomyocytes and endothelial cells, which are difficult to be isolated from the patient tissues (Choi et al., 2009; Hu et al., 2010; Lian et al., 2012; Liu et al., 2016; Spence et al., 2011; Sullivan et al., 2010; Takebe et al., 2013; Zhang et al., 2009). These differentiated cells derived from patient-specific iPSCs could recapitulate the disease phenotypes in variety of diseases that included monogenic disorders which are caused by single gene defect e.g. Lesch-Nyhan disease and fragile X syndrome (Eiges et al., 2007; Urbach et al., 2004), complex disorders 
resulting from multiple gene defects, such as autism spectrum disorder and schizophrenia (Brennand et al., 2011; DeRosa et al., 2012), early-onset disorders diagnosed during childhood such as Turner and Down syndrome (Morris et al., 1999), and late-onset disorders which occur in adult life such as Parkinson and Alzheimer diseases (Devine et al., 2011; Yagi et al., 2011). Modeling cardiac diseases with hiPSC technology also represents an attractive and viable approach to study cardiac disease progression, signaling pathways as well as drug mechanism of action in human cardiomyocytes (Figure 3). Some human cardiac diseases e.g. long-QT syndrome, Barth syndrome and catecholaminergic polymorphic ventricular tachycardia have been successfully modeled with cardiomyocytes derived from patient-specific iPSCs (Davis et al., 2012; Dudek et al., 2016; Itzhaki et al., 2011).

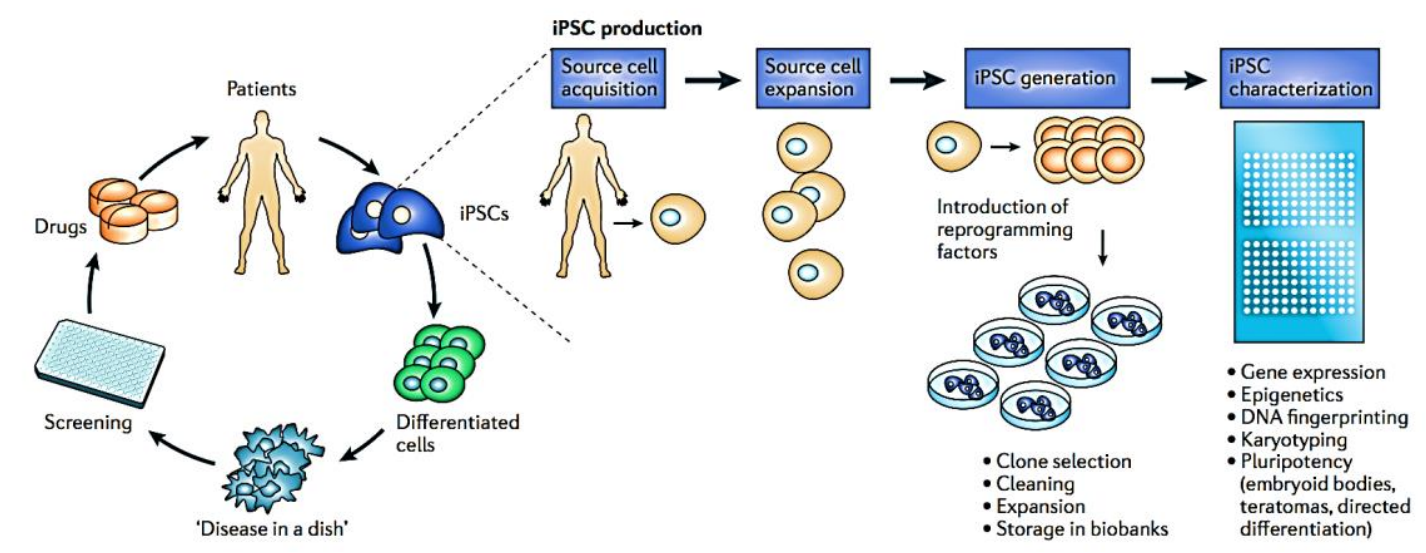

Figure 3. iPSCs as a platform for disease modeling and drug screening. Somatic cells from patients are harvested and reprogrammed to yield iPSCs. The cells are then differentiated into specific cell types that are used to model the disease condition. These differentiated cells are then used in compound screening and drug validation. The figure was taken from (Grskovic et al., 2011).

The iPSCs platform has also been used to recapitulate the disease phenotype and to study molecular mechanisms of autophagy and lysosome defective diseases, particularly the lysosomal storage disorder. For instance, Gaucher disease (GD) is a genetic disorder caused by mutations in the $\beta$-glucocerebrosidase gene, which lead to the abnormal accumulation of glucocerebroside in spleen, liver, kidneys, lungs, brain and bone marrow. Neuronal cells derived from GD-specific iPSCs showed widespread lysosomal depletion, autophagosome accumulation and significantly downregulated expression of TFEB 
(transcription factor EB), a master regulator of lysosome biogenesis (Awad et al., 2015). Another example is Pompe disease, caused by the deficiency of lysosomal acid alpha-glucosidase, which is an autosomal recessive metabolic disorder. Cardiomyocytes derived from Pompe disease-specific iPSCs showed a Golgi-based glycosylation deficit but without autophagosome accumulation detected by LC3II (Raval et al., 2015). Taken together, these disease-specific iPSCs provide researchers a suitable human disease model to understand the disease progression and possibly discover new biomarkers for drug discovery.

\subsubsection{Patient-specific iPSCs used for drug screening}

Drug screening strategies using iPSCs have been developed and applied to high throughput screening (Avior et al., 2016) (Figure 3). The studies were firstly performed based on the diseased mouse neurons, and then the selected drugs were further tested on motor neurons and astrocytes derived from ALS patient-specific iPSCs (Barmada et al., 2014; Dimos et al., 2008; Sances et al., 2016). New candidates of drugs such as Taxol, isoxazole, NAB2, GW5074 and co-enzyme Q10 for neuron dysfunctional diseases were discovered by using the patient-specific iPSC models since 2009 (Cooper et al., 2012; Lee et al., 2009; Ren et al., 2015; Ryan et al., 2013; Shcheglovitov et al., 2013). Niemann-Pick disease type C (NPC) is a lysosomal storage disease with abnormal accumulation of cholesterol and glycolipids. A potential new drug 2-hydroxypropyl- $\gamma$-cyclodextrin was discovered to target this disease by reducing cholesterol accumulation in hepatocyte-like cells and neural progenitors derived from NPC-specific iPSCs in vitro (Soga et al., 2015). Cardiomyocytes derived from iPSCs, which exhibit the similar characteristics of human embryonic cardiomyocytes, including structural, molecular, functional properties and response with specific drugs, provide a good platform for screening the anti-arrhythmic drugs and exploring the cardiac toxic tests (Liang et al., 2013).

\subsubsection{Genomic editing of iPSCs using the CRISPR/Cas9 technique}

To achieve the specific gene modification, the CRISPR/Cas9 (clustered regularly 
interspaced short palindromic repeat (CRISPR) and CRISPR associated endonuclease 9 (Cas9)) system in the prokaryotic was quickly adapted for genomic editing in eukaryotic systems including Drosophila, C. elegans, zebrafish, mouse, rat and human (Cho et al., 2013; Cong et al., 2013; DiCarlo et al., 2013; Gratz et al., 2013; Hwang et al., 2013). Genomic DNA editing in mammalian cells were also achieved by using the zinc-finger nuclease (ZFN) and transcription-activating protein (TALEN) systems, which are applied for inserting or rescuing mutation sites in genes for understanding their functions (Bibikova et al., 2003; Bibikova et al., 2002; Boch et al., 2009; Moscou and Bogdanove, 2009). When compared with the ZFN and TALEN technologies, the CRISPR/Cas9 technique has higher efficiency to generate nucleotide(s) insertion or deletion via non-homologous end joining (NHEJ) and homologous recombination (HR), which conveniently allows for new discovery of gene functions and molecular mechanisms of human genetic diseases. Using gene-editing techniques such as CRISPR/Cas9 and specific guide RNA (sgRNA), single nucleotide gene correction was introduced into hiPSCs, which was to model the disease carrying specific gene mutations (Gonzalez et al., 2014) and to "correct" causative mutation in monogenic recessive disorders such as cystic fibrosis, sickle-cell anemia or Duchene muscular dystrophy (Mali et al., 2013b). The CRISPR/Cas9 gene correction approach was introduced to rectify an aberrant copy of the cystic fibrosis transmembrane conductor receptor (CFTR) gene in intestinal stem cells of cystic fibrosis patients, resulting in "gene-corrected" stem cells from these patients, which were able to form stable epithelial organoids (Schwank et al., 2013).

\subsubsection{Engineered heart muscle generated from iPSCs-derived cardiomyocytes}

Engineered heart muscles (EHMs) are 3-dimensional cardiac tissues generated from the assembly of cardiomyocytes and cardiac supportive non-myocytes in a hydrogel mixture. These tissues provide necessary environmental cues to improve the maturation of hiPSC-derived cardiomyocytes, including sarcomere structure and arrangement, gene expression, contractility and particularly maturation of the ion channels (Eder et al., 2016). Compared to monolayer cardiomyocyte cultures, EHMs are physiologically more relevant, as they possess similar complex structures and functions of the native heart 
tissue (Mathur et al., 2016). Previous data show that EHMs generated from hiPSCs-derived cardiomyocytes could be used to demonstrate the contractile function of normal and diseased cardiac tissues in vitro (Mannhardt et al., 2016).

\subsection{Aim of this study}

In this study, we recruited a patient (3-month-old) with Vici syndrome. Previous gene analysis revealed that the patient carries a homozygous intronic mutation in EPG5 (c.4952+1G>A). Based on sequencing of cDNA derived from patient fibroblast cultures, this mutation was predicted to result in a frame shift and premature stop codon insertion, p.Phe1604Glyfs*20 (Cullup et al. 2013). Conformational analysis of fibroblasts derived from this patient also showed autophagosome accumulation due to the impaired autophagosome-lysosome fusion (Cullup et al., 2013). However, it was not elucidated whether the cardiomyocytes (CMs) showed the same phenotype in comparison to fibroblasts and if the impaired autophagy was the direct consequence of the EPG5 mutation.

The aims of this thesis are to study Vici syndrome associated cardiomyopathy using patient-specific iPSCs as a disease model in vitro and to elucidate the molecular mechanisms of the autophagy pathway in CMs. The main objectives are:

- Generation of iPSCs from the patient with Vici syndrome carrying the EPG5 mutation $($ c. $4952+1 \mathrm{G}>\mathrm{A})$.

- "Proof of pluripotency" of the generated patient-specific iPSCs.

- Analysis of the disease phenotype and the autophagy pathway in CMs derived from Vici-iPSCs (Vici-iPSC-CMs).

- Correction of the mutation in EPG5 in Vici-iPSCs by using the CRISPR/Cas9 technology. 


\section{Materials and Methods}

\subsection{Materials}

\subsubsection{Oligonucleotides}

\begin{tabular}{|c|c|c|c|c|}
\hline Name & Sequence (5'-3') & Annealing Temp $\left({ }^{\circ} \mathrm{C}\right)$ & Cycle & Size (bp) \\
\hline EPG5_1 & $\begin{array}{l}\text { for: AACCGCAGCTCTTCGGGA AT } \\
\text { rev: CTAATGCCAACTTTCTGAATCCCA }\end{array}$ & 56 & 35 & $\begin{array}{l}336(\mathrm{Ctr}) \\
223 \text { (Vici) }\end{array}$ \\
\hline EPG5_2 & $\begin{array}{l}\text { for: GGCATGCATAAGAATGAAGCCA } \\
\text { rev: TAAATGGGGATGGCTGTGTTG }\end{array}$ & 56 & 37 & 330 \\
\hline EPG5_3 & $\begin{array}{l}\text { for: CCT TGGCATGGAGTTGGT TTG } \\
\text { rev: CTCACAATTCAGGGGTCAGC }\end{array}$ & 58 & 36 & 1076 \\
\hline$F O X D 3$ & $\begin{array}{l}\text { for: GTGAAGCCGCCTTACTCGTAC } \\
\text { rev: CCGAAGCTCTGCATCATGAG }\end{array}$ & 58 & 38 & 353 \\
\hline GAPDH & $\begin{array}{l}\text { for: AGAGGCAGGGATGATGTTCT } \\
\text { rev: TCTGCTGATGCCCCCATGTT }\end{array}$ & 58 & 30 & 258 \\
\hline GDF3 & $\begin{array}{l}\text { for: TTCGCTTTCTCCCAGACCAAGGTTTC } \\
\text { rev: TACATCCAGCAGGTTGAAGTGAAC } \\
\text { AGCACC }\end{array}$ & 58 & 32 & 311 \\
\hline LIN28 & $\begin{array}{l}\text { for: AGTAAGCTGCACATGGAAGG } \\
\text { rev: ATTGTGGCTCAATTCTGTGC }\end{array}$ & 58 & 36 & 410 \\
\hline МYH7 & $\begin{array}{l}\text { for: GAAGGTGAGTGTCCCAGAGG } \\
\text { rev: CTTGTAGTCGATGTTCCCCG }\end{array}$ & 58 & 32 & 289 \\
\hline MYL2 & $\begin{array}{l}\text { for: GGCGAGTGAACGTGAAAAAT } \\
\text { rev: CAGCATTTCCCGAACGTAAT }\end{array}$ & 56 & 30 & 200 \\
\hline$N K X 2.5$ & $\begin{array}{l}\text { for: GGCCTCAATCCCTACGGTTA } \\
\text { rev: CACGAGAGTCAGGGAGCTGT }\end{array}$ & 60 & 30 & 308 \\
\hline $\mathrm{SOX} 2$ & $\begin{array}{l}\text { for: ATGCACCGCTACGACGTGA } \\
\text { rev: CTTTTGCACCCCTCCCATTT }\end{array}$ & 58 & 30 & 437 \\
\hline
\end{tabular}




\section{TNNT2 for: GACAGAGCGGAA AAGTGGGA \\ rev: TGA AGGAGGCCAGGCTCTAT}

EPG5_1: primers for detecting the expression of EPG5 on mRNA level; EPG5_2: primers for detecting the mutation site of EPG5 in genomic DNA; EPG5_3: primers for cleavage assay to test the efficiency of CRISPR/Cas9/gRNA; GAPDH: Glycerinaldehyde-3-phosphate-dehydrogenase; FOXD3: Forkhead box D3; GDF3: Growth differentiation factor-3; MYH7: Myosin heavy chain 7; MYL2: Myosin light chain 2; NKX2.5: NK2 homeobox 5; TNNT2: Troponin T type 2.

Single-stranded oligonucleotides of EPG5 were used for correction of the EPG5 mutation in the CRISPR/Cas9 experiments, as following:

AGTGAAGCAGTTGCAAGCAGAAGCTGCTAAACCACCAAGCTTAAATATTGTAGAAGCTGCTGT ACATGCAGAAAACTTGATCACGTAAGTTTTGCAGGCATTATAATTTGAAATACTGTTTTG

\subsubsection{Primary antibodies}

\begin{tabular}{|c|c|c|c|c|}
\hline Antigen & Type & Supplier & Catalog No. & Dilution \\
\hline AFP & Polyclonal rabbit IgG & Dako & A0008 & IF: $1: 200$ \\
\hline AKT & Monoclonal rabbit IgG & CST & 4691 & WB: 1:1000 \\
\hline AKT S473 & Monoclonal rabbit IgG & CST & 9271 & WB: 1:1000 \\
\hline AKT T308 & Monoclonal rabbit IgG & CST & 2965 & WB: 1:1000 \\
\hline COXIV & Monoclonal rabbit IgG & $\mathrm{CST}$ & 4850 & IF: $1: 100$ \\
\hline cTNT & Monoclonal mouse IgG & $\begin{array}{l}\text { Thermo } \\
\text { Fisher } \\
\text { Scientific }\end{array}$ & MS295PABX & IF: $1: 200$ \\
\hline CX43 & Polyclonal rabbit IgG & Abcam & Ab11370 & IF: $1: 200$ \\
\hline FOXO3 & Monoclonal rabbit IgG & $\mathrm{CST}$ & 2467 & WB:1:1000 \\
\hline $\begin{array}{l}\text { FOXO3 T32 } \\
\text { FOXO1 T24 }\end{array}$ & Monoclonal rabbit IgG & CST & 9464 & WB: 1:1000 \\
\hline GAPDH & Polyclonal rabbit IgG & Abcam & ab9485 & WB: $1: 1000$ \\
\hline LAMP1 & Monoclonal mouse IgG & Abcam & ab25630 & IF: $1: 100$ \\
\hline LC3 & Monoclonal rabbit IgG & $\mathrm{CST}$ & 3868 & IF: $1: 100, W B: 1: 1000$ \\
\hline LIN28 & Polyclonal goat IgG & $\mathrm{R} \& \mathrm{D}$ & AF3757 & IF: $1: 300$ \\
\hline MLC2V & Monoclonal rabbit IgG & Protein tech & 10906-1-AP & IF: $1: 200$ \\
\hline mTOR & Monoclonal rabbit IgG & $\mathrm{CST}$ & 2983 & WB: 1:1000 \\
\hline mTOR S2448 & Monoclonal rabbit $\operatorname{IgG}$ & CST & 2971 & WB: 1:1000 \\
\hline
\end{tabular}




\begin{tabular}{|c|c|c|c|c|}
\hline NANOG & Polyclonal rabbit IgG & $\begin{array}{l}\text { Thermo } \\
\text { Fisher } \\
\text { Scientific }\end{array}$ & PA1-097 & IF: $1: 100$ \\
\hline NBR1 & Monoclonal mouse IgG & Abcam & $\mathrm{ab} 55474$ & IF: $1: 100, \mathrm{WB}: 1: 1000$ \\
\hline OCT4 & Polyclonal goat IgG & $\mathrm{R} \& \mathrm{D}$ & AF1759 & IF: $1: 40$ \\
\hline P62 & Monoclonal rabbit IgG & Abcam & ab109012 & IF:1:100, WB:1:1000 \\
\hline P70S6K & Monoclonal rabbit IgG & CST & 2708 & WB: $1: 1000$ \\
\hline P70S6K T389 & Monoclonal rabbit IgG & CST & 9234 & WB: 1:1000 \\
\hline $\begin{array}{l}\text { Phalloidin- } \\
\text { iFluor } 647\end{array}$ & $\begin{array}{l}\text { Fluorescent iFluor647 dye } \\
\text { for F-ACTIN }\end{array}$ & Abcam & ab176759 & IF: $1: 100$ \\
\hline SMA & Monoclonal mouse IgG & Sigma & A2547 & IF: $1: 1000$ \\
\hline SSEA4 & Monoclonal mouse IgG & $\begin{array}{l}\text { Thermo } \\
\text { Fisher } \\
\text { Scientific }\end{array}$ & MA1-021 & IF: $1: 100$ \\
\hline$\alpha$-ACTININ & Monoclonal mouse IgG & Sigma & A7811 & IF: $1: 1000$ \\
\hline$\beta$-III-TUBULIN & Monoclonal mouse IgG & Covance & MMS-435P & IF: $1: 1000$ \\
\hline
\end{tabular}

AFP: Alpha-1-fetoprotein; AKT S473: AKT serine 473; AKT T308: AKT threonine 308; COXIV: Cytochrome c oxidase subunit IV; cTNT: Cardiac troponin T; CX43: Connexin 43; FoxO3: Forkhead box O3; FoxO3 T32: FOXO3 threonine 32; FOXO1: Forkhead box O1; FOXO1 T24: FOXO1 threonine 24; LAMP1: Lysosomal associated protein 1; MLC2V: Myosin light chain 2V; P70S6K: Ribosomal protein S6 kinase, 70 kDa, polypeptide 1; P70S6K T389: P70S6K threonine 389; Phalloidin-iFluor 647: Far-red fluorescent dye for F-ACTIN; SMA: Smooth muscle actin; SSEA4: Stage-specific embryonic antigen-4.

\subsubsection{Secondary antibodies}

\begin{tabular}{|c|c|c|c|}
\hline Name & Supplier & Catalog No. & Dilution \\
\hline Cy3-conjugated donkey-a-goat IgG & Jackson ImmunoResearch & $705-166-147$ & IF: $1: 600$ \\
\hline Cy3-conjugated goat- $\alpha$-mouse IgG & Jackson ImmunoResearch & $115-165-068$ & IF: $1: 600$ \\
\hline Cy3-conjugated goat- $\alpha$-rabbit IgG & Jackson ImmunoResearch & $111-165-003$ & IF: $1: 300$ \\
\hline FITC-conjugated goat- $\alpha$-mouse IgG & Jackson ImmunoResearch & $115-096-072$ & IF: $1: 300$ \\
\hline FITC-conjugated goat- $\alpha$-rabbit IgG & Jackson ImmunoResearch & $111-095-045$ & IF: $1: 300$ \\
\hline HRP-conjugated anti-mouse IgG & Sigma & RABHRP2 & WB: $1: 3000$ \\
\hline HRP-conjugated anti-rabbit IgG & Sigma & RABHRP1 & WB: $1: 3000$ \\
\hline
\end{tabular}




\subsubsection{Commercial kits}

\begin{tabular}{lll}
\hline Name & Supplier & Catalog No. \\
\hline Alkaline phosphatase staining kit & Sigma & 86R-1KT \\
\hline Genomic cleavage detection kit & Thermo Fisher Scientific & A24372 \\
Human stem cell nucleofector kit 2 & Lonza & VPH-5022 \\
\hline TransIT-293 reagent & Mirus & 41094613 \\
\hline QIAprep Spin Maxiprep kit & Qiagen & 12163 \\
\hline QIAprep Spin Miniprep kit & Qiagen & 27104 \\
\hline QIAquick gel extraction kit & Qiagen & 28704 \\
\hline Maxwell 16 cell DNA purification kit & Promega & AS1020 \\
\hline SV total RNA isolation kit & Promega & Z3105 \\
\hline
\end{tabular}

\subsubsection{Cell lines}

\begin{tabular}{ll}
\hline Name & Cell type \\
\hline isWT1BLD2 & Human iPSCs from a healthy donor generated in Dr. Guan's lab \\
\hline MEFs & Mouse embryonic fibroblasts isolated from 15-17-day-old embryos of NMRI mice \\
\hline Vici FB & Human fibroblasts from the Vici patient obtained from Dr. Gautel's lab \\
\hline iBM76.3 & Human iPSCs from a healthy donor generated in Dr. Guan's lab \\
\hline WTD2-1 & Human iPSCs from a healthy donor generated in Dr. Guan's lab \\
\hline iVici1.2 & Human iPSCs from the Vici patient generated in this study \\
\hline iVici1.3 & Human iPSCs from the Vici patient generated in this study \\
\hline iVici1.5 & Human iPSCs from the Vici patient generated in this study \\
\hline
\end{tabular}

\subsubsection{Basal media, growth factors, cytokines and drugs for cell culture}

\begin{tabular}{llc}
\hline Name & Supplier & Catalog No. \\
\hline $\mathbf{0 . 0 5 \%}$ Trypsin-EDTA & Life technologies & 25300054 \\
$\mathbf{0 . 2 5 \%}$ Trypsin-EDTA & Life technologies & 25200056 \\
$\mathbf{2 . 5 \%}$ Trypsin & Life technologies & 15090046 \\
TrypLE Express & Life technologies & 1734778 \\
\hline
\end{tabular}




\begin{tabular}{|c|c|c|}
\hline 4',6-Diamidino-2-phenylindole (DAPI) & Sigma & D9542 \\
\hline $60 \%$ Sodium DL-lactate solution & Sigma & L4263 \\
\hline 7.5\% Bovine albumin fraction $\mathrm{V}$ (BSA) & Life technologies & 15260037 \\
\hline Albumin, human recombinant & Sigma & A0237 \\
\hline Bafilomycin A1 & Sigma & B1793 \\
\hline Basic fibroblast growth factor (bFGF) & PeproTech & $100-18 \mathrm{~B}$ \\
\hline Bovine serum albumin (BSA) & Sigma & F7524 \\
\hline B27 supplement & Life technologies & 17504044 \\
\hline CHIR99021 & Merck Millipore & 361559 \\
\hline Collagenase type II & Worthington & LS004176 \\
\hline Collagenase type IV & Worthington & LS004189 \\
\hline Dimethyl sulfoxide (DMSO) & Sigma & D2650 \\
\hline Dulbecco's modified Eagle medium (DMEM) & Life technologies & 11960044 \\
\hline DMEM/F-12 with GlutaMax & Life technologies & 31331028 \\
\hline Dulbecco's phosphate buffered saline (DPBS) & Life technologies & 14190094 \\
\hline Essential 8 (E-8) basal medium & Life technologies & A1517001 \\
\hline Ethylenediaminetetraacetic acid (EDTA) & Sigma & E6758 \\
\hline Fetal bovine serum (FBS) & Sigma & F7524 \\
\hline Gelatin & Sigma & 48720 \\
\hline Geltrex & Life technologies & A1413301 \\
\hline HEPES Buffer (1 M, pH 7.0-7.6) & Sigma & H0887 \\
\hline Inhibitor of WNT production-2 (IWP2) & Merck Millipore & 68167 \\
\hline $\begin{array}{l}\text { Iscove's modified Dulbecco's medium (IMDM) } \\
\text { with GlutaMax }\end{array}$ & Life technologies & 31980022 \\
\hline IMDM with HEPES without GlutaMax & HyClone & SH30259.01 \\
\hline Isopropanol & Merck Millipore & 1096341000 \\
\hline Isoproterenol & Sigma & $\mathrm{I} 2760$ \\
\hline Knockout serum replacement (KSR) & Life technologies & 10828028 \\
\hline L-ascorbic acid 2-phosphate & Sigma & A8960 \\
\hline L-glutamine, $100 x$ & Life technologies & 25030081 \\
\hline
\end{tabular}




\begin{tabular}{|c|c|c|}
\hline Lipofectamine 2000 & life technologies & 11668019 \\
\hline Medium 199 & Sigma & M 4530 \\
\hline Mitomycin C & Serva Electrophoresis & 29805.02 \\
\hline Monothioglycerol (MTG) & Sigma & M6145 \\
\hline Non-essential amino acids (NEAA), 100x & Life technologies & 11140035 \\
\hline Opti-MEM® I reduced serum medium & Life technologies & 31985070 \\
\hline Penicillin-streptomycin solution, 100x & Life technologies & 15140122 \\
\hline Phenylephrine & Sigma & P6126 \\
\hline Polybrene (hexadimethrine bromide) & Sigma & 107689 \\
\hline Pro survival factor (PSF) & Merck Millipore & 529659 \\
\hline Rapamycin & Sigma & R0395 \\
\hline Rat collagen & \multicolumn{2}{|c|}{ Produced by the Wolfram-Hubertus } \\
\hline $\begin{array}{l}\text { Roswell Park Memorial Institute (RPMI) } 1640 \text { medium with } \\
\text { GlutaMax }\end{array}$ & Life technologies & 72400021 \\
\hline RPMI 1640 (w/o glucose) & Life technologies & 11879020 \\
\hline StemPro accutase cell dissociation reagent & Life technologies & A1110501 \\
\hline STEMCCA vector & $\begin{array}{l}\text { provided by Prof. } \\
\text { University School of } \mathrm{N}\end{array}$ & $\begin{array}{l}\text { Kotton, Boston } \\
\text { edicine }\end{array}$ \\
\hline Thiazovivin (TZV) & Merck Millipore & 420220 \\
\hline Trypsin powder & Life technologies & 27250018 \\
\hline Versene solution & Life technologies & 15040066 \\
\hline$\beta$-mercaptoethanol & Serva Electrophoresis & 28625 \\
\hline
\end{tabular}

STEMCCA vector: The Cre-excisable STEMCCA vector contains four transcription factors OCT4, KLF4, $S O X 2$, and $c-M Y C$, which separated by the self-cleaving $2 \mathrm{~A}$ peptide and internal ribosome entry site sequences. This vector also contains two loxP sites for removing the transgenic genes with Cre-mediated excisable system following reprogramming (Somers et al., 2010).

\subsubsection{Stock solutions for cell culture}

\begin{tabular}{ll}
\hline Drugs & Components \\
\hline bFGF $50 \mu \mathrm{g} / \mathrm{ml}$ & $1 \mathrm{mg} \mathrm{bFGF}$ \\
\hline
\end{tabular}




\begin{tabular}{|c|c|}
\hline & $\begin{array}{l}20 \mathrm{ml} 5 \mathrm{mM} \text { Tris } \\
\text { Store aliquots at }-20^{\circ} \mathrm{C}\end{array}$ \\
\hline bFGF $5 \mu \mathrm{g} / \mathrm{ml}$ & $\begin{array}{l}200 \mu \mathrm{l}(50 \mu \mathrm{g} / \mathrm{ml} \text { stock }) \\
1800 \mu \mathrm{l} 0.1 \% \mathrm{BSA} / \mathrm{DPBS} \\
\text { Store at } 4^{\circ} \mathrm{C} \text { not longer than two weeks }\end{array}$ \\
\hline$\beta$-mercaptoethanol $(100 x)$ & $\begin{array}{l}35 \mu \mathrm{l} \beta \text {-mercaptoethanol } \\
50 \mathrm{ml} \text { DPBS } \\
\text { Filtrate with } 0.22 \mu \mathrm{m} \text { Steriflip filter }\end{array}$ \\
\hline $1 \% \mathrm{BSA} / \mathrm{DPBS}$ & $\begin{array}{l}1 \mathrm{ml} \mathrm{7.5 \%} \mathrm{BSA} \\
6.5 \mathrm{ml} \mathrm{DPBS}\end{array}$ \\
\hline $0.1 \%$ BSA/DPBS & $\begin{array}{l}0.1 \mathrm{ml} \mathrm{7.5 \%} \mathrm{BSA} \\
7.4 \mathrm{ml} \mathrm{DPBS}\end{array}$ \\
\hline 0.1\% Trypsin-EDTA & $\begin{array}{l}0.1 \mathrm{~g} \text { trypsin } \\
0.1 \mathrm{~g} \text { EDTA } \\
100 \mathrm{ml} \mathrm{H}_{2} \mathrm{O}\end{array}$ \\
\hline $1 \%$ Gelatin & $\begin{array}{l}1 \mathrm{~g} \text { gelatin } \\
100 \mathrm{ml} \mathrm{H}_{2} \mathrm{O} \\
\text { Autoclave, and store at } 4^{\circ} \mathrm{C}\end{array}$ \\
\hline Collagenase II (46 U/ml) & $\begin{array}{l}1 \mathrm{mg} \text { collagenase II ( } 46 \mathrm{U} / \mathrm{mg}) \\
1 \mathrm{ml} \text { RPMI } \\
\text { Store aliquots at }-20^{\circ} \mathrm{C}\end{array}$ \\
\hline Collagenase IV $(200 \mathrm{U} / \mathrm{ml})$ & $\begin{array}{l}1 \mathrm{mg} \text { collagenase IV (200 U/mg) } \\
1 \mathrm{ml} \mathrm{DMEM} / \mathrm{F}-12 \\
\text { Store aliquots at }-20^{\circ} \mathrm{C}\end{array}$ \\
\hline CHIR99021 (12 mM) & $\begin{array}{l}5 \mathrm{mg} \text { CHIR99021 } \\
894 \mu \mathrm{l} \text { DMSO } \\
\text { Store aliquots at }-20^{\circ} \mathrm{C}\end{array}$ \\
\hline IWP2 (5 mM) & $\begin{array}{l}10 \mathrm{mg} \text { IWP2 } \\
4.28 \mathrm{ml} \mathrm{DMSO} \\
37^{\circ} \mathrm{C} 10 \mathrm{~min}\end{array}$ \\
\hline
\end{tabular}




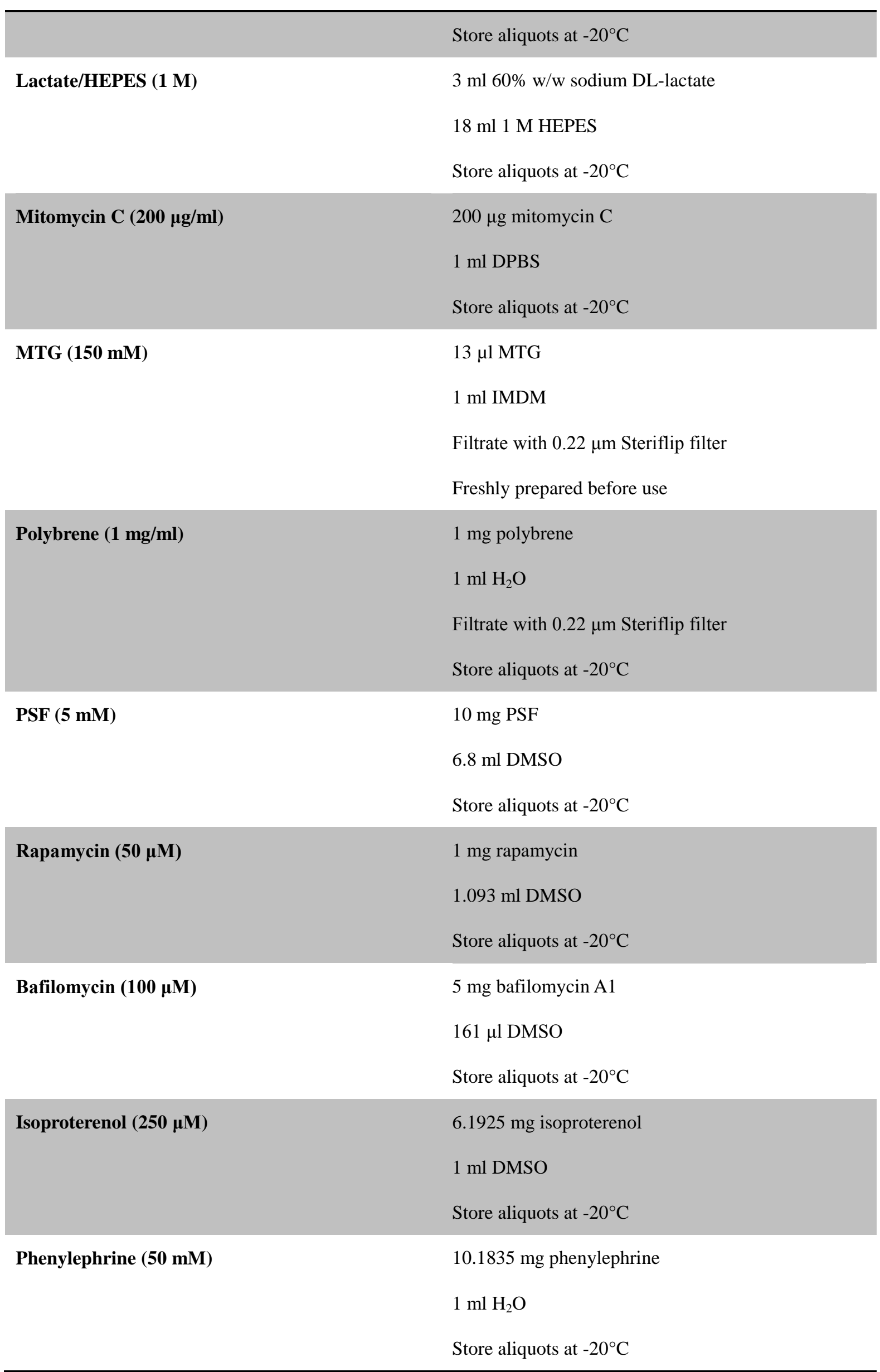




\subsubsection{Cell culture media}

\begin{tabular}{|c|c|}
\hline Medium Name & Components \\
\hline Cardiac culture medium & $\begin{array}{l}\text { RPMI } 1640 \text { medium with GlutaMax } \\
\text { 1x B27 supplement }\end{array}$ \\
\hline Cardiac differ medium & $\begin{array}{l}\text { RPMI } 1640 \text { medium with GlutaMax } \\
0.05 \% \text { albumin } \\
0.02 \% \text { ascorbic acid }\end{array}$ \\
\hline Cardiac selection medium & $\begin{array}{l}\text { RPMI } 1640 \text { without glucose } \\
2 \text { ml } 1 \text { M lactate }\end{array}$ \\
\hline E-8 freezing medium & $\begin{array}{l}\text { E- } 8 \text { medium } \\
20 \% \text { DMSO } \\
\text { PSF }(5 \mu \mathrm{M})\end{array}$ \\
\hline E-8 medium & $\begin{array}{l}\text { E-8 basal medium } \\
\text { E-8 essential supplement }\end{array}$ \\
\hline Freezing medium & $\begin{array}{l}\text { DMEM } \\
20 \% \text { FBS } \\
2 \% \text { DMSO }\end{array}$ \\
\hline hEHM medium & $\begin{array}{l}\text { IMDM with HEPES without GlutaMax } \\
20 \% \text { FBS } \\
\text { 1x L-glutamine } \\
1 \times \text { NEAA } \\
1 \times \beta \text {-mercaptoethanol }\end{array}$ \\
\hline hES medium & $\begin{array}{l}\text { DMEM/F-12 with GlutaMax } \\
15 \% \mathrm{KSR} \\
1 \times \text { NEAA } \\
\text { bFGF }(10-20 \mathrm{ng} / \mathrm{ml})\end{array}$ \\
\hline HFBM & $\begin{array}{l}\text { DMEM } \\
10 \% \text { FBS } \\
1 \times \beta \text {-mercaptoethanol }\end{array}$ \\
\hline
\end{tabular}




\begin{tabular}{ll}
\hline HFF medium & 1x NEAtamine \\
& DMEM \\
& $20 \%$ FBS \\
& $1 x$ Glutamine \\
& $1 x$ NEAA \\
& bFGF (10-20 ng/ml) \\
& IMDM with GlutaMax \\
& $20 \%$ FBS \\
Iscove Medium & $1 x$ NEAA \\
& MTG (150 mM) \\
\hline MEF medium & $10 \%$ FBS \\
\hline
\end{tabular}

\subsubsection{Chemicals and reagents for molecular biological analysis}

\begin{tabular}{|c|c|c|}
\hline Name & Supplier & Catalog No. \\
\hline Acetone & J.T.Baker & 408 \\
\hline Agarose & Peqlab & $35-1020$ \\
\hline Ammonium persulfate (APS) & Sigma & A3678 \\
\hline Boric acid & Sigma & 15663 \\
\hline Bromphenol blue & Sigma & 1062 \\
\hline Calcium chloride $\left(\mathrm{CaCl}_{2}\right)$ & Sigma & C1016 \\
\hline $\begin{array}{l}\text { Color prestained protein standard, broad range }(11-245 \\
\mathrm{kDa})\end{array}$ & NEB & P7712 \\
\hline Diethylpyrocarbonate (DEPC) water & Ambion & AM9915G \\
\hline DNA agar & Peqlab & A 3477.0500 \\
\hline dNTP mix & Bioline & Bio39029 \\
\hline Ethanol & J.T.Baker & L216-07 \\
\hline Ethidium bromide & Carl Roth & 2218 \\
\hline
\end{tabular}




\begin{tabular}{|c|c|c|}
\hline Fluoromount-G & eBioscience & 00-4938-02 \\
\hline GeneRulerTM 100 bp Plus DNA ladder & $\begin{array}{l}\text { Thermo Fisher } \\
\text { Scientific }\end{array}$ & 0321 \\
\hline Glacial acetic acid & Merck Millipore & 1.00063 .1000 \\
\hline Glucose & Sigma & G8270 \\
\hline Glycine & Carl Roth & $\mathrm{T} 873$ \\
\hline GoTaq DNA polymerase & Promega & M3175 \\
\hline Hind III restriction enzyme & NEB & R0104S \\
\hline Hydrochloric acid (HCl) & J.T.Baker & 2612 \\
\hline Immobilon Western chemiluminescent HRP substrate & Merck Millipore & WBKLS0500 \\
\hline LB agar & Applichem & 416106.1210 \\
\hline Lysogeny broth (LB) powder & Applichem & A0954 \\
\hline Magnesium chloride $\left(\mathbf{M g C l}_{2}\right)$ & Sigma & M8266 \\
\hline Methanol & J.T.Baker & 8402 \\
\hline MuLV reverse transcriptase $(50 \mathrm{U} / \mu \mathrm{l})$ & Life technologies & N808-0018 \\
\hline Nonfat dry milk & Sigma & M7409 \\
\hline NP 40 & $\begin{array}{l}\text { Thermo Fisher } \\
\text { Scientific }\end{array}$ & 85125 \\
\hline Oligo d(T)16 (50 $\mu \mathrm{M})$ & Life technologies & N808-0128 \\
\hline Paraformaldehyde (PFA) & Sigma & 158127 \\
\hline Polyvinylidene fluoride (PVDF) membrane & Millipore & IPVH00010 \\
\hline Ponceau S solution & Sigma & P7170 \\
\hline Potassium chloride (KCl) & Sigma & P9333 \\
\hline Quick coomassie blue staining solution & Generon & GEN-QC-STAIN-1L \\
\hline RNase inhibitor (20 U/ $\mu \mathrm{l})$ & Life technologies & N808-0119 \\
\hline Sodium bicarbonate $\left(\mathrm{NaHCO}_{3}\right)$ & Sigma & S5761 \\
\hline Sodium chloride $(\mathrm{NaCl})$ & Carl Roth & P3957 \\
\hline Sodium dodecyl sulfate (SDS) & Carl Roth & 2326 \\
\hline Sodium hydroxide $(\mathrm{NaOH})$ & J.T.Baker & 3722 \\
\hline Sodium phosphate monobasic $\left(\mathrm{NaH}_{2} \mathrm{PO}_{4}\right)$ & Sigma & S8282 \\
\hline Tetramethylethylenediamine (TEMED) & Carl Roth & 2367 \\
\hline
\end{tabular}




\begin{tabular}{|c|c|c|c|}
\hline Tris & Carl Roth & & 5429 \\
\hline Triton X100 & Sigma & & X100 \\
\hline Tween 20 & Bio Rad & & $170-6531$ \\
\hline Urea & Carl Roth & & 3941 \\
\hline Western blot gel & $\begin{array}{l}\text { Thermo } \\
\text { Scientific }\end{array}$ & Fisher & NW04120BOX \\
\hline
\end{tabular}

\subsubsection{Buffers and solutions for molecular biological analysis}

\begin{tabular}{|c|c|}
\hline Names & Components \\
\hline \multirow[t]{2}{*}{$1.5 \%$ Agar gel } & $1.5 \mathrm{~g}$ DNA agar \\
\hline & $100 \mathrm{ml} \mathrm{TB}$ buffer \\
\hline \multirow[t]{2}{*}{$10 \%$ APS } & $10 \mathrm{~g}$ APS \\
\hline & $100 \mathrm{ml} \mathrm{H}_{2} \mathrm{O}$ \\
\hline \multirow[t]{2}{*}{ 4\% PFA } & $4 \mathrm{~g}$ PFA \\
\hline & $100 \mathrm{ml} \mathrm{DPBS}$ \\
\hline \multirow[t]{5}{*}{ Blue loading buffer $(100 \mathrm{ml}, \mathbf{5 x})$} & $31.25 \mathrm{ml} 1 \mathrm{M}$ Tris- $\mathrm{HCl}(\mathrm{pH} 6.8)$ \\
\hline & $10 \mathrm{~g} \mathrm{SDS}$ \\
\hline & $5 \mathrm{mg}$ bromphenol blue \\
\hline & $57 \mathrm{ml}$ glycerin $(87 \%)$ \\
\hline & $11 \mathrm{ml} \beta$-mercaptoethanol \\
\hline \multirow[t]{2}{*}{$\mathrm{CaCl}_{2}(2.25 \mathrm{M})$} & $165.57 \mathrm{~g} \mathrm{CaCl}_{2}$ \\
\hline & $500 \mathrm{ml} \mathrm{H}_{2} \mathrm{O}$ \\
\hline \multirow[t]{5}{*}{ Cell lysis buffer (RIPA buffer) } & $150 \mathrm{mM} \mathrm{NaCl}$ \\
\hline & $1.0 \% \mathrm{NP}-40$ \\
\hline & $0.5 \%$ sodium deoxycholate \\
\hline & $0.1 \%$ SDS \\
\hline & $50 \mathrm{mM}$ Tris \\
\hline \multirow[t]{2}{*}{0.6 mM DAPI } & $0.21 \mathrm{mg}$ DAPI \\
\hline & $1 \mathrm{ml} \mathrm{H}_{2} \mathrm{O}$ \\
\hline Fixation buffer & $7 \mathrm{ml}$ methanol (ice-cold) \\
\hline
\end{tabular}


$3 \mathrm{ml}$ glacial acetic acid (ice-cold)

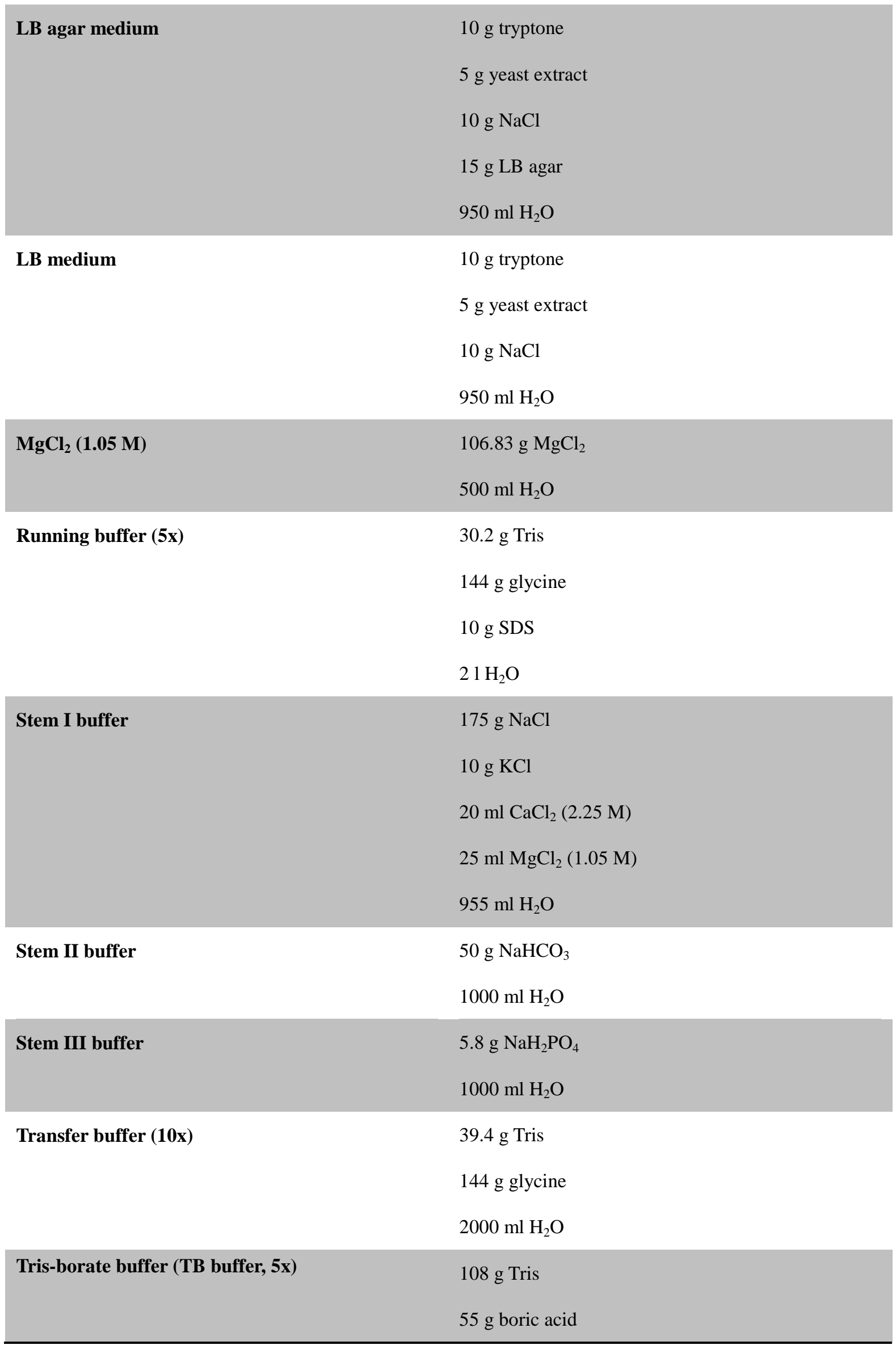




\begin{tabular}{|c|c|}
\hline & $2000 \mathrm{ml} \mathrm{H}{ }_{2} \mathrm{O}$ \\
\hline \multirow[t]{4}{*}{ Tris-buffered saline with Tween 20 (TBST, 1x) } & $48.4 \mathrm{~g}$ Tris \\
\hline & $58.48 \mathrm{NaCl}$ \\
\hline & $10 \mathrm{ml}$ Tween 20 \\
\hline & $1990 \mathrm{ml} \mathrm{H}{ }_{2} \mathrm{O}$ \\
\hline \multirow[t]{3}{*}{1 M Tris-HCl (pH 6.8) } & $121.14 \mathrm{~g}$ Tris \\
\hline & $\mathrm{HCl}$ to $\mathrm{pH} 6.8$ \\
\hline & Adding $\mathrm{H}_{2} \mathrm{O}$ to $1000 \mathrm{ml}$ \\
\hline \multirow[t]{4}{*}{ Tris-SDS (4x, pH 6.8) } & $6.05 \mathrm{~g}$ Tris \\
\hline & $0.4 \mathrm{~g}$ SDS \\
\hline & $\mathrm{HCl}$ to $\mathrm{pH} 6.8$ \\
\hline & Adding $\mathrm{H}_{2} \mathrm{O}$ to $100 \mathrm{ml}$ \\
\hline \multirow[t]{4}{*}{ Tris-SDS (4x, pH 8.8) } & $45.5 \mathrm{~g}$ Tris \\
\hline & $1 \mathrm{~g} \mathrm{SDS}$ \\
\hline & $\mathrm{NaOH}$ to $\mathrm{pH} 8.8$ \\
\hline & Adding $\mathrm{H}_{2} \mathrm{O}$ to $250 \mathrm{ml}$ \\
\hline \multirow[t]{5}{*}{ Tyrode buffer } & $40 \mathrm{ml}$ Stem I buffer \\
\hline & $38 \mathrm{ml}$ Stem II buffer \\
\hline & $10 \mathrm{ml}$ Stem III buffer \\
\hline & $1 \mathrm{~g}$ glucose \\
\hline & $100 \mathrm{mg}$ L-ascorbic acid \\
\hline
\end{tabular}

pH value regulation: dissolve the chemicals in $\mathrm{H}_{2} \mathrm{O}$ and adjust the $\mathrm{pH}$ value with appropriate volume of concentrated $\mathrm{HCl}$ or $\mathrm{NaOH}$, thereafter, bring to final volume with $\mathrm{H}_{2} \mathrm{O}$.

\subsubsection{Lab equipment and other materials}

\begin{tabular}{lll}
\hline Equipment and other materials & Manufacturer & Catalog No. \\
\hline $\mathbf{0 . 2 2} \boldsymbol{\mu m}$ Steriflip filter & Merck Millipore & SLGSM33SS \\
$\mathbf{1 . 5} \mathbf{~ m l ~ b r o w n ~ t u b e ~}$ & Eppendorf & 0030120.191 \\
$\mathbf{1 . 5} \mathbf{~ m l ~ t u b e}$ & Eppendorf & AM12400 \\
$\mathbf{1 0} \mathbf{~ m}$ pipette & Sarstedt & 86.1254 .001 \\
\hline
\end{tabular}




\begin{tabular}{|c|c|c|}
\hline $15 \mathrm{ml}$ falcon & Sarstedt & 62.554 .002 \\
\hline $2 \mathrm{ml}$ glass pipette & Hirschmann & 1000141 \\
\hline $25 \mathrm{ml}$ pipette & Sarstedt & 86.1685 .001 \\
\hline $5 \mathrm{ml}$ glass pipette & Hirschmann & 1101153 \\
\hline $5 \mathrm{ml}$ pipette & Sarstedt & 86.1253 .001 \\
\hline $50 \mathrm{ml}$ falcon & Sarstedt & 62.547 .004 \\
\hline $50 \mathrm{ml}$ filter falcon & Merck Millipore & SCGP00525 \\
\hline Benchtop Tissue Processor 1020 & Leica Biosystems & 1491020US01 \\
\hline Cell culture (untreated) 6-cm dish & Sarstedt & 82.1194 .500 \\
\hline Cell culture $10-\mathrm{cm}$ dish & CytoOne Starlab & CC7682-3394 \\
\hline Cell culture 12 -well plate & CytoOne Starlab & CC7682-7512 \\
\hline Cell culture 24-well plate & CytoOne Starlab & CC7682-7524 \\
\hline Cell culture $3-\mathrm{cm}$ dish & CytoOne Starlab & CC7682-3340 \\
\hline Cell culture 48-well plate & $\begin{array}{l}\text { Thermo Fischer } \\
\text { Scientific }\end{array}$ & 150687 \\
\hline Cell culture 6-cm dish & CytoOne Starlab & CC7682-3359 \\
\hline Cell culture 6-well plate & CytoOne Starlab & CC7682-7506 \\
\hline Cell culture 96-well plate & $\begin{array}{l}\text { Thermo Fischer } \\
\text { Scientific }\end{array}$ & 167008 \\
\hline Cell culture bench & HERA & 51013961 \\
\hline Cell culture incubator & HERA & 5103568 \\
\hline Cell Scraper & Sarstedt & 83.1830 \\
\hline Centrifuge & $\begin{array}{l}\text { Thermo Fischer } \\
\text { Scientific }\end{array}$ & 5415 \\
\hline ChemiDoc $^{\mathrm{TM}}$ MP system & Bio-Rad & 1708280 \\
\hline Casy cell counter & Roche & \\
\hline DNA gel running cassette & BioRad & 1704402 \\
\hline FACSCanto II & $\mathrm{BD}$ & \\
\hline FACS Aria II & $\mathrm{BD}$ & \\
\hline Filter $50 \mu \mathrm{m}$ & $\mathrm{BD}$ & 340629 \\
\hline Filtertips 0.1-10 $\mu \mathrm{l}$ & CytoOne Starlab & S1120-3810 \\
\hline
\end{tabular}




\begin{tabular}{|c|c|c|}
\hline Filtertips 100-1000 $\mu \mathrm{l}$ & CytoOne Starlab & S1122-1830 \\
\hline Filtertips 1-100 $\mu \mathrm{l}$ & CytoOne Starlab & S1120-1840 \\
\hline Glass bottle $100 \mathrm{ml}$ & Th.Geier & 9.072011 \\
\hline Glass bottle $1000 \mathrm{ml}$ & Th.Geier & 9.072016 \\
\hline Glass bottle $250 \mathrm{ml}$ & Th.Geier & 9.072012 \\
\hline Glass bottle $50 \mathrm{ml}$ & Th.Geier & 9.072020 \\
\hline Glass bottle $500 \mathrm{ml}$ & Th.Geier & 9.072 .015 \\
\hline Laser scanning microscope & Zeiss & LSM510 \\
\hline Nanodrop spectrophotometer & $\begin{array}{l}\text { Thermo Fischer } \\
\text { Scientific }\end{array}$ & ND-2000 \\
\hline PCR machine & Sensoquest & 012-103 \\
\hline pH meter level 1 & Inolab & ba12217e \\
\hline Pipette boy & Accu-jet pro & 5281090 \\
\hline Water bath machine & $\begin{array}{l}\text { Thermo Fischer } \\
\text { Scientific }\end{array}$ & 18005A-1CEQ \\
\hline WB running cassette & BioRad & 1658004 \\
\hline
\end{tabular}

\subsubsection{Software}

\begin{tabular}{ll}
\hline Name & Name \\
\hline Adobe Illustrator 2015 & Alpha image \\
\hline AMON/BMON & Adobe Photoshop 2015 \\
\hline Carl Zeiss AxioVision Rel 4.8 & Chromas \\
\hline Flowing software & GraphPad Prism 6 \\
\hline Image Lab 5 & Image J \\
\hline Zen 2009 & \\
\hline
\end{tabular}

\subsection{Methods}

\subsubsection{Cell culture}

All cell cultures were incubated at $37^{\circ} \mathrm{C}$ and $5 \% \mathrm{CO}_{2}$ under humidified conditions in incubators. 


\subsubsection{Cultivation of human fibroblasts}

Fibroblasts from the patient with Vici syndrome were obtained from Dr. Mathias Gautel's lab (Kings College, London) and cultured in HFBM supplemented with $10 \mathrm{ng} / \mathrm{ml}$ bFGF. For passaging, fibroblasts were rinsed with $0.1 \%$ trypsin-EDTA once following aspiration of the culture medium. The cells were then incubated with $0.1 \%$ trypsin-EDTA for $3 \mathrm{~min}$ at $37^{\circ} \mathrm{C}$ to allow cell detachment. Equal volume of HFBM was subsequently added to neutralize the action of trypsin. The total cell suspension was collected and allowed to pellet in a centrifuge at $200 \times \mathrm{g}$ for $5 \mathrm{~min}$ at room temperature. After aspirating the supernatant, the cells were plated into new cell culture dishes at a ratio of 1:3-1:6 depending on the culture density.

\subsubsection{Generation of iPSCs from fibroblasts derived from the patient with Vici syndrome}

To generate Vici-iPSCs, patient-derived fibroblasts were reprogrammed by transduction of the cells with the STEMCCA lentivirus system containing OCT4, SOX2, KLF4 and c-MYC in a single plasmid, as described previously (Streckfuss-Bomeke et al., 2013). Briefly, $24 \mathrm{~h}$ prior to the transduction, fibroblasts were passaged into a 12-well plate at a seeding density of $6 \times 10^{4}$ cells per well. The fibroblasts were transduced with lentivirus particles in HFBM at MOI (multiplicity of infection) of 1.0, 2.0 and 3.0 supplemented with $1 \mu \mathrm{g} / \mathrm{ml}$ polybrene for $24 \mathrm{~h}$. Polybrene, a cationic polymer, was used to increase the efficiency of DNA virus infection into eukaryotic cells (Davis et al., 2002). The virus containing medium was then removed $24 \mathrm{~h}$ post transduction and fresh HFBM supplemented with $10 \mathrm{ng} / \mathrm{ml}$ bFGF was added. Subsequent medium was changed daily until day 7 post transduction, and the transduced cells were passaged onto 6-cm dishes that were coated with $0.2 \mathrm{mg} / \mathrm{ml} \mathrm{Geltrex}$ at $37^{\circ} \mathrm{C}$ for $30 \mathrm{~min}$ or at $4^{\circ} \mathrm{C}$ overnight, at a ratio of 1:4, 1:5 and 1:6 in HFBM with $10 \mathrm{ng} / \mathrm{ml}$ bFGF. $24 \mathrm{~h}$ later, medium was changed to E-8 medium that was used daily until colonies with typical morphology of human pluripotent stem cells appeared in the culture dish. The single hiPSC colonies were manually cut with sterilized glass pipettes into small pieces and plated onto Geltrex-coated 12-well plates under the cell culture bench. These colonies were cultured in E-8 medium and used in 
this study.

\subsubsection{Feeder-free cultivation of hiPSCs}

For feeder-free cultivation of hiPSCs, cell culture dishes were coated with Geltrex for 30 min at $37^{\circ} \mathrm{C}$ or overnight at $4^{\circ} \mathrm{C}$ before use. Both Ctr-iPSCs (hiPSCs derived from healthy donors) and Vici-iPSCs were subsequently cultured on these Geltrex-coated dishes in E-8 medium. Upon reaching 80-90\% confluence, hiPSCs were passaged onto new dishes. Briefly, hiPSCs were washed once with Versene and incubated with fresh Versene for 4-5 $\mathrm{min}$ at room temperature. After aspirating the Versene solution, the dissociated cells were re-suspended in E-8 medium with $5 \mu \mathrm{M}$ PSF and seeded into the new dishes. The medium was then changed daily with E-8 medium.

\subsubsection{Isolation of mouse embryonic fibroblasts}

MEFs were isolated from the 15-17-day-old mouse embryos. After the pregnant mouse was rinsed in $70 \%$ ethanol, the abdomen was opened and the embryos were washed with DPBS twice. Then the head and red organs (such as heart, liver and intestinal) were removed. The remained tissues were rinsed in $0.2 \%$ trypsin and minced into pieces with a sterile razor blade. The tissues were then transferred into a $50 \mathrm{ml}$ Erlenmeyer flask containing a stir bar with fresh $0.2 \%$ trypsin and stirred on magnetic stirrer for $30 \mathrm{~min}$. The digested tissues were pipetted up and down several times to aid further dissociation of tissue clumps. The suspension was filtered through a sieve or a careen, neutralized with equal volume of MEF medium and finally spun at $300 \times g$ in a centrifuge. After the supernatant was aspirated, the cells were re-suspended in warm MEF medium and plated in tissue culture dishes. The cells were cultured in MEF medium until 80-90\% confluent and frozen down for future usage. These tubes were then denoted as passage $0(\mathrm{P} 0)$.

\subsubsection{Cultivation and inactivation of mouse embryonic fibroblasts}

MEFs were cultured on cell culture dishes in MEF medium for only 4 passages to support the growth of hiPSCs. To ensure that the MEFs remain growth arrested during the proliferation of hiPSCs, MEFs were incubated with $10 \mu \mathrm{g} / \mathrm{ml}$ mitomycin $\mathrm{C}$ for $3-4 \mathrm{~h}$ at 
$37^{\circ} \mathrm{C}$ before use. Thereafter, the cells were rinsed with DPBS 3 times, digested with $0.1 \%$ trypsin-EDTA, and neutralized with MEF medium. MEFs with an appropriate cell density were seeded onto $0.1 \%$ gelatin-coated dishes (e.g. $1.5 \times 10^{5}$ cells $/ \mathrm{cm}^{2}$ ) and cultured in MEF medium until use.

\subsubsection{Cultivation of hiPSCs on feeder layer}

To cultivate hiPSCs on feeder layer, hiPSCs were rinsed with basal DMEM/F12 twice and digested with collagenase IV $(200 \mathrm{U} / \mathrm{ml})$ for $3 \mathrm{~min}$ at room temperature. The cells were then cut into small clusters using the cell scraper, gently pipetted twice and plated on the dishes with inactivated MEFs in hES medium. Thereafter, repeated passage of the cells was performed every 6-7 days. Differentiated cell regions characterized by the presence of cystic areas were mechanically removed during medium change daily.

\subsubsection{Spontaneous differentiation of hiPSCs in vitro}

HiPSCs cultured on feeder layer were used for the spontaneous differentiation in vitro to prove their pluripotency. Briefly, hiPSCs were washed twice with basal DMEM/F12 and digested with collagenase IV $(200 \mathrm{U} / \mathrm{ml})$ for $4 \mathrm{~min}$. The cell colonies were cut into bigger pieces, re-suspended in hES medium and transferred to low attachment cell culture dishes. During the first day, cell clusters round up into compact cell aggregates termed "embryoid bodies" (EBs). The next day, EBs were collected and spun at $250 \mathrm{x} g$ for 2 min in a centrifuge. Supernatant was carefully aspirated and the EBs were cultured in Iscove medium until day 8 . On day 8 , EBs were plated onto $0.1 \%$ gelatin-coated cell culture dishes for further differentiation. At day 33, differentiated cells were briefly rinsed with DPBS and fixed with $4 \%$ PFA for $20 \mathrm{~min}$ at room temperature for immunofluorescence staining.

\subsubsection{Direct differentiation of hiPSCs into cardiomyocytes}

Feeder-free culture of hiPSCs in E-8 medium were used for the direct differentiation into CMs. Briefly, hiPSCs were first passaged into Geltrex-coated 12-well plates and allowed to proliferate for 2-3 days till $80 \%$ cell confluence in each well to provide sufficient cells 
for cardiac differentiation. Cardiac differentiation was initiated by replacing E-8 medium with cardiac differ medium supplemented with 4-6 $\mu$ M CHIR99021 (day 0), an inhibitor of GSK $3 \beta$ to induce hiPSCs differentiating into mesendoderm progenitors. After $24 \mathrm{~h}$ (on day 1), CHIR99021 was removed and cells were maintained in cardiac differ medium. On day 3, the cardiac differ medium was supplemented with $2.5 \mu \mathrm{M}$ IWP2, the Wnt antagonist to induce the commitment of mesendoderm progenitors to a cardiac fate. On day 5 , the medium was replaced with cardiac differ medium. From day 8 , the medium was changed to cardiac culture medium and the first beating cardiomyocytes were observed on day 10-14. To obtain purified cardiomyocytes, the beating cardiomyocyte cultures at day 16-20 were enzymatically dissociated into single cells. Briefly, the cardiomyocytes were first incubated with collagenase II $(46 \mathrm{U} / \mathrm{ml})$ at $37^{\circ} \mathrm{C}$ for $2 \mathrm{~h}$ to allow the detachment of cardiomyocytes. Detached cells were collected into 15-ml falcon and spun down at $250 \times \mathrm{g}$ for $5 \mathrm{~min}$ in a centrifuge. In order to obtain the single cardiomyocytes, the cells were then treated with $0.25 \%$ trypsin for $8 \mathrm{~min}$ at $37^{\circ} \mathrm{C}$. The action of trypsin was neutralized by adding FBS and the total cell suspension was pelleted in a centrifuge. The resultant cell pellet was suspended in cardiac culture medium and seeded onto new Geltrex-coated dishes. After 4-day cultivation in cardiac culture medium, fresh medium was changed to cardiac selection medium (with supplementation of lactate instead of glucose) for 4-6 days to metabolically select cardiomyocytes, thereby increasing the purity of the $\mathrm{CM}$ culture. Afterwards, these purified cardiomyocytes were maintained in cardiac culture medium for another 2-3 months for further experimental studies.

\subsubsection{Cryopreserved and thawing of the cultivated cells}

To cryopreserve fibroblasts and hiPSCs, the cells were digested into single cells as previously described, accordingly. Afterwards cell pellets were re-suspended in freezing medium or E-8 freezing medium according to the culture and then transferred into the cryovials. The cryovials containing the cells were kept overnight at $-80^{\circ} \mathrm{C}$ in a freezing container with isopropanol and then transferred into liquid nitrogen vapor for extended storage. 
To thaw the cells, frozen cells were retrieved and placed in a $37^{\circ} \mathrm{C}$ warm water bath. After thawing, the cells were immediately immersed into cold fresh medium and spun at $250 \mathrm{x} g$ for $5 \mathrm{~min}$ in a centrifuge. The cells were re-suspended and plated onto new cell culture dishes in cell culture medium, respectively.

\subsubsection{Nucleofection of hiPSCs}

The custom CRISPR/Cas9-GFP plasmid (Figure 4) with EPG5-specific guide RNA (sgRNA) was designed together with Sigma. The plasmid DNA was amplified in E. coli bacterial cultures in LB medium with $50 \mu \mathrm{g} / \mathrm{ml}$ kanamycin for $36 \mathrm{~h}$. DNA was isolated using the QIAprep Spin Maxiprep kit according to the manufacturer's instructions. After analysis of the cleavage efficacy of the designed sgRNA in HEK293T cells (see 2.2.10), the CRISPR/Cas9-GFP plasmid DNA together with single-stranded oligonucleotides of EPG5 (2.1.1) was introduced into Vici-iPSCs.

For transfection, hiPSCs were digested with $0.05 \%$ Trypsin-EDTA for $3 \mathrm{~min}$ at $37^{\circ} \mathrm{C}$ until the cells detached. Pre-warmed DMEM with FBS was added to neutralize the dissociation action of trypsin-EDTA. Cell numbers were counted with cell counting chamber. $3 \times 10^{6}$ cells were spun down at $200 \times \mathrm{g}$ for $5 \mathrm{~min}$. After the supernatant was removed, the cell pellet was re-suspended in a mixture of nucleofection buffer A ( $82 \mu 1)$ and B $(18 \mu \mathrm{l})$ containing $1.5 \mu \mathrm{g}$ CRISPR/Cas9-GFP plasmid DNA and $30 \mathrm{ng}$ singlestranded oligonucleotides of EPG5. The sample was then transferred to $0.4 \mu \mathrm{m}$ cuvette and electrified using the appropriate Nucleofector program B-016 that was initially determined for hESCs using the human stem cell nucleofector kit 2 . The transfected cells were transferred to E-8 medium containing $5 \mu \mathrm{M}$ PSF for recovery for $10 \mathrm{~min}$ at $37^{\circ} \mathrm{C}$. Finally, the cells were seeded on the Geltrex-coated 6-well plate with E-8 medium containing $5 \mu \mathrm{M}$ PSF. 


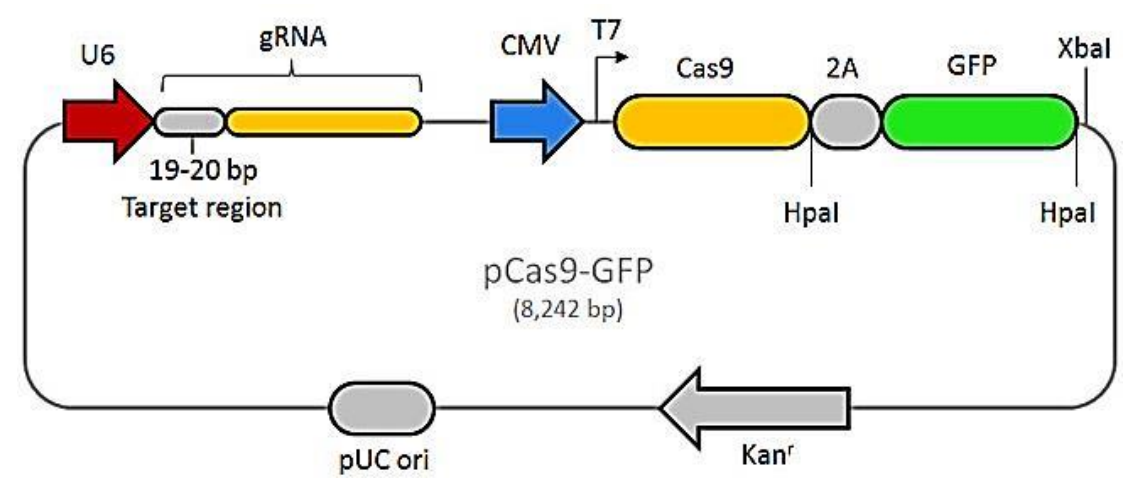

Figure 4. The scheme of CRISPR/Cas9-GFP vector. Guide RNA (gRNA) and Cas9 protein are expressed in a single vector, which is controlled by the promoter U6 and CMV, respectively. The GFP fused with 2A peptide to Cas9 enables to track the efficiency of transfection and to enrich the transfected cells through FACS sorting. Figure was taken from Sigma homepage.

\subsubsection{Transfection of hiPSC-derived cardiomyocytes}

The hiPSC-derived cardiomyocytes (hiPSC-CMs) were dissociated into single cells and cultured as monolayers as described above. Cardiomyocytes were transfected with lipofectamine 2000 according to the manufacturer's recommendations. The LC3-GFP-RFP plasmid DNA (Figure 5) was obtained from Prof. Dr. Lucie Carrier (Department of Experimental Pharmacology and Toxicology, University Medical Center Hamburg-Eppendorf). The plasmid DNA and lipofectamine 2000 reagent were individually mixed with Opti-MEM and allowed to incubate at room temperature for 10 min. Then the DNA mixture was transferred into the lipofectamine 2000 mixture and incubated for $5 \mathrm{~min}$. Thereafter, the DNA-lipid complex was gently layered dropwise onto the cultured cardiomyocytes. The cells were fixed by $4 \%$ PFA for confocal images $48 \mathrm{~h}$ after transfection.

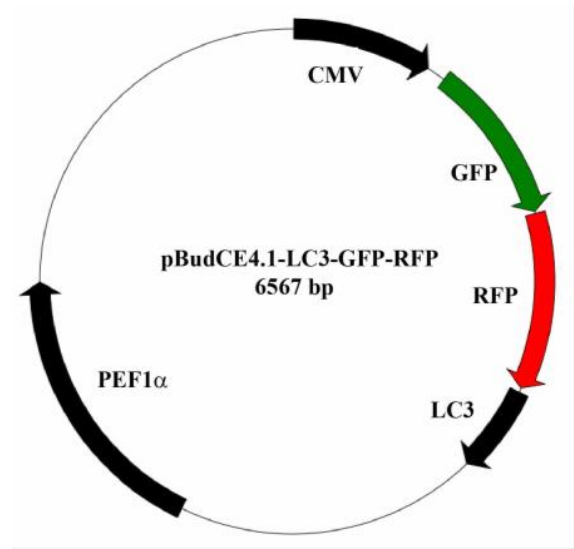

Figure 5. The scheme of pBudCE4.1-LC3-GFP-RFP vector. GFP and RFP are coupled with LC3 under the CMV promoter. 


\subsubsection{Alkaline phosphatase staining}

Alkaline phosphatase is a highly expressed protein in pluripotent cells and its detection in cell colonies represents a strong evidence for the success of iPSC generation. In this study, alkaline phosphatase staining was performed according to the manufacturer's instructions. Briefly, $1 \mathrm{ml}$ sodium nitrite solution was first mixed with $1 \mathrm{ml} \mathrm{FBB}$-alkaline solution for $2 \mathrm{~min}$, and then diluted in $45 \mathrm{ml} \mathrm{H}_{2} \mathrm{O}$. Prior to the staining, the AS-BI alkaline solution was freshly added to the prepared mixture. The hiPSC cultures were washed twice with DPBS and fixed by citrate-acetone-formaldehyde solution at room temperature for $30 \mathrm{~s}$. Then the cells were rinsed gently in $\mathrm{H}_{2} \mathrm{O}$ for $45 \mathrm{~s}$. The alkaline-dye mixed solution was added and incubated for 15 min in the dark. Finally, the cells were washed with $\mathrm{H}_{2} \mathrm{O}$ and left to dry at room temperature. The positive cells were stained in red and the images were taken with the Zeiss microscope.

\subsubsection{Teratoma formation and analysis}

The hiPSCs cultured on feeder layer were used for the teratoma formation in vivo. Vici-iPSCs were dissociated in collagenase IV for 2-3 min before mechanically dissection into small clusters using a cell scraper. These clusters were collected with $2 \mathrm{ml} \mathrm{hES}$ medium and subcutaneously injected into the immunodeficient mice without recombination activating gene 2 and gamma $C\left(\mathrm{RAG}^{-{ }_{-}} \mathrm{\gamma c}^{-/-}\right)$. Teratomas were collected 60 days after injection of iPSCs and fixed with phosphate buffered formalin ( $\mathrm{pH} 7.0$ ) for $4 \mathrm{~h}$ at room temperature. After washing with DPBS and $\mathrm{H}_{2} \mathrm{O}$, the teratomas were dehydrated and embedded in paraffin using the Benchtop Tissue Processor 1020. Thereafter, the samples were sectioned into $6 \mu \mathrm{m}$ sections with a microtome and subjected to histological staining with hematoxylin and eosin $(\mathrm{H} \& \mathrm{E})$. The animal and histological experiments were performed by the technicians in Dr. Guan's lab. All H\&E images were obtained with brightfield microscopy.

\subsubsection{Isolation of genomic DNA and DNA sequencing}

Genomic DNA from hiPSCs was extracted using the automatic Maxwell 16 cell DNA purification kit under the manufacturer's instructions. The cell pellets were suspended in 
$300 \mu 1 \mathrm{H}_{2} \mathrm{O}$ and transferred into well 1 of the cartridge while the plunger was placed in well 7 of the cartridge. These cartridges containing samples and plungers were transferred onto the Maxwell 16 platform. The protocol for cells was then chosen and purification based on the magnetic sorting was activated. The DNA was eluted with $300 \mu 1$ TE buffer. Concentration of the genomic DNA was measured at 260/280 nm using the NanoDrop 2000 spectrophotometer. The isolated DNA was stored in the $-80^{\circ} \mathrm{C}$ freezer.

For the DNA sequencing, the specific regions of EPG5 were amplified by PCR from the genomic DNA using the specific primer sets. To verify the mutation in the generated Vici-iPSCs, the primer set EPG5_2 (see section 2.1.1) was used. To determine whether the homologous recombination happened in the genome of Vici-iPSCs after nucleofection with the CRISPR/Cas9-GFP plasmid and single-stranded oligonucleotides of EPG5, the primer set EPG5_3 (see section 2.1.1) was used. The PCR product was electrophoretically separated on a $1.5 \%$ agar gel, subsequently excised with a scalpel and extracted using the QIAquick gel extraction kit according to the manufacturer's instructions. The isolated PCR product was then sent for DNA sequencing.

\subsubsection{Gene expression analysis}

\subsubsection{Isolation of RNA from cultured cells}

Total RNA was isolated using the SV total RNA isolation system according to the manufacturer's instructions. First, cells were lysed in 400-1000 $\mu 1$ RNA lysis buffer and transferred to a new Eppendorf tube where $400 \mu \mathrm{l}$ of ethanol was added to precipitate the DNA. Then, $800 \mu \mathrm{l}$ mixture was pipetted into a spin column and spun at $16100 \mathrm{x} g$ for 1 min in a centrifuge. $800 \mu \mathrm{l}$ RA washing buffer was added into the column and spun for an additional $1 \mathrm{~min}$. The samples were treated with a mixture of supplied core buffer (45 $\mu \mathrm{l}), 0.09 \mathrm{M} \mathrm{MnCl}_{2}(5 \mu \mathrm{l})$ and deoxyribonuclease I (DNase I, $\left.5 \mu \mathrm{l}\right)$ at room temperature for $15 \mathrm{~min}$. After $200 \mu \mathrm{l}$ DNase stop solution was added, the samples were spun and washed twice with the provided rinsing buffer. Thereafter, the RNA was eluted with nuclease-free $\mathrm{H}_{2} \mathrm{O}$ and stored at $-80^{\circ} \mathrm{C}$. The concentration of RNA was measured using the NanoDrop 2000 spectrophotometer at A260/A280. The isolated RNA was 
subsequently used for reverse transcription.

\subsubsection{Reverse transcription of RNA to cDNA}

200 ng isolated RNA was used to synthesize complementary DNA (cDNA) with reverse transcriptase. The reagents were listed in the following table.

\begin{tabular}{ll}
\hline Reagents & Volume $(20 \boldsymbol{\mu l}$ in total $)$ \\
\hline DEPC water and RNA & $10.2 \mu \mathrm{l}$ \\
\hline $\mathbf{1 0} \mathbf{x}$ PCR buffer II & $2 \mu \mathrm{l}$ \\
$\mathbf{2 5} \mathbf{~} \mathbf{M M ~ M g C l}_{\mathbf{2}}$ & $4 \mu \mathrm{l}$ \\
\hline $\mathbf{1 0} \mathbf{~ m M ~ d N T P s}$ & $0.8 \mu \mathrm{l}$ \\
$\mathbf{5 0} \boldsymbol{\mu M}$ Oligo d(T) 16 & $1 \mu \mathrm{l}$ \\
\hline $\mathbf{R N a s e}$ inhibitor $(\mathbf{2 0} \mathbf{U} / \boldsymbol{\mu l})$ & $1 \mu \mathrm{l}$ \\
\hline MuL V reverse transcriptase $(\mathbf{5 0} \mathbf{U} / \boldsymbol{\mu l})$ & $1 \mu \mathrm{l}$ \\
\hline
\end{tabular}

Reverse transcription was performed in a PCR cycler. The mixture was subjected to a cycle of thermal gradient of $22^{\circ} \mathrm{C}$ for $10 \mathrm{~min}, 42^{\circ} \mathrm{C}$ for $50 \mathrm{~min}, 99^{\circ} \mathrm{C}$ for $10 \mathrm{~min}$ and a cooling phase at $4^{\circ} \mathrm{C}$. The resultant cDNA was then stored at $-20^{\circ} \mathrm{C}$ for further usage.

\subsubsection{Polymerase chain reaction}

Polymerase chain reaction (PCR) was used to amplify the specific DNA fragments. The traditional PCR reaction consists of denaturing of DNA strands, annealing of the forward and reverse primers and synthesis of DNA. The annealing temperature was determined with the primers of selected genes (2.1.1). The reagents were listed in the following table.

\begin{tabular}{ll}
\hline Reagents & Volume $(25 \boldsymbol{\mu l}$ in total $)$ \\
\hline cDNA & $1 \mu \mathrm{l}$ \\
\hline 10x Green GoTaq reaction PCR buffer & $2.5 \mu \mathrm{l}$ \\
\hline $\mathbf{1 0}$ mM dNTPs & $1 \mu \mathrm{l}$ \\
\hline Forward primer $(\mathbf{1 0 0} \boldsymbol{\mu M})$ & $1 \mu \mathrm{l}$ \\
\hline Reverse primer $(\mathbf{1 0 0} \boldsymbol{\mu M})$ & $1 \mu \mathrm{l}$ \\
\hline GoTaq DNA polymerase $(\mathbf{5} \mathbf{U} / \boldsymbol{\mu l})$ & $0.1 \mu \mathrm{l}$ \\
\hline DEPC water & $18.4 \mu \mathrm{l}$ \\
\hline
\end{tabular}


The reaction was accomplished in a PCR cycler with the following program:

$$
\begin{aligned}
& 95{ }^{\circ} \mathrm{C} \quad 3 \mathrm{~min} \\
& 95^{\circ} \mathrm{C} \quad 30 \mathrm{~s} \\
& 50-60{ }^{\circ} \mathrm{C} 30 \mathrm{~s} \\
& 72{ }^{\circ} \mathrm{C} \quad 30 \mathrm{~s} \\
& 72{ }^{\circ} \mathrm{C} \quad 10 \mathrm{~min} \\
& 4{ }^{\circ} \mathrm{C}
\end{aligned}
$$

The PCR product was verified using gel electrophoresis on a 1.5\% DNA agar gel.

\subsubsection{Agar gel electrophoresis}

The DNA fragments amplified by PCR were loaded in a 1-2\% DNA agar gel and subjected to gel electrophoresis. The percentage of the gel was chosen depending on the size of the PCR product of the selected gene region. Briefly, agar was weighed and dissolved in $1 \mathrm{x}$ TB buffer. The agar was melted and allowed to cool gradually until $60^{\circ} \mathrm{C}$. In order to visualize the amplified PCR bands under ultraviolet (UV) light, ethidium bromide was added before the agar mixture was poured into horizontal gel chamber with appropriate number of gel combs. The agar gel was polymerized after $20 \mathrm{~min}$. Thereafter the amplified PCR products as well as the DNA molecular weight marker were loaded into the wells of the agar block. The gel was subjected to an electrophoresis of 70-120 volts for 20-40 min. DNA bands were detected and characterized under UV trans-illumination and the images were captured using the Alpha image software.

\subsubsection{Western blot}

\subsubsection{SDS polyacrylamide gel electrophoresis}

SDS polyacrylamide gel electrophoresis (PAGE) was used to separate proteins with different molecular weights. The 4\%-20\% ladder commercial SDS gels were purchased from Bio-Rad. Gels were assembled into the Bio-Rad chambers before immersing into the SDS running buffers. The samples with lysis buffer and pre-stain protein markers were loaded on the gel. The gels were then subjected to gel electrophoresis that runs at 70 volts for $20 \mathrm{~min}$ and a further $1-1.5 \mathrm{~h}$ at 120 volts. The protein bands were then identified based on the expected size compared with the protein ladders. The gels were 
counterstained with coomassie dye staining or used for further investigation of Western blot.

\subsubsection{Protein transfer and detection}

The proteins on the SDS-PAGE were electrophoretically transferred onto a PVDF membrane at $400 \mathrm{~mA}$ for $1 \mathrm{~h}$ in the blotting chamber filled with the transferring buffer and cooled with ice. The successfully transferred membrane was then washed three times with TBST buffer and subsequently treated with 5\% non-fat dried milk for $1 \mathrm{~h}$ to block any unspecific binding. Primary antibody was diluted with 5\% non-fat dried milk and overlaid onto the membrane and incubated at $4^{\circ} \mathrm{C}$ overnight. Thereafter, the membrane was washed with TBST buffer for 5 times at $5 \mathrm{~min}$ per rinse. The HRP-coupled secondary antibody was then overlaid onto the membrane and incubated at room temperature for $1 \mathrm{~h}$. Following repeated washing steps of the membrane, the blots were incubated with detection buffer (ECL prime) for chemiluminescent signal recording. The detection buffer $\mathrm{A}$ and $\mathrm{B}$ were mixed at a ratio of 1:1 and layered over the membrane to allow for the signal development. Exposure and images of the developed membrane blots were recorded using VersaDoc coupled with ImageLab 5 software. All washing steps were performed at room temperature.

\subsubsection{Immunofluorescence staining and analysis of images}

For immunofluorescence staining, cells were fixed with 4\% PFA for $20 \mathrm{~min}$ at room temperature. Thereafter, cells were washed with DPBS 3 times to remove traces of the fixative. The cells were then blocked with $1 \%$ BSA for $30 \mathrm{~min}$ at room temperature. For target antigens located in the cytoplasm and nuclei, the cells were permeabilized with $0.1 \%$ Triton X100 for 10 min. After washing with DPBS for 3 times, the primary antibody was diluted in $1 \% \mathrm{BSA}$ and then incubated at $4{ }^{\circ} \mathrm{C}$ overnight. The next day, cells were washed with DPBS for 3 times. The fluorescence dye-coupled secondary antibody was applied to the cells and incubated for $1 \mathrm{~h}$ at room temperature in the dark. The nucleus was counter-stained with $300 \mathrm{nM}$ DAPI (1:2000 v/v dilutions from the $0.6 \mathrm{mM}$ stock) for 15 min at room temperature. Thereafter, the antibody-labeled samples were washed with 
DPBS for 3 times and mounted onto glass slides with Fluoromount GM. Images were captured by using the confocal microscope. Brightfield images were also captured and used as indicated in corresponding figures.

\subsubsection{Measurement of cell surface in hiPSC-CMs}

Images of randomly selected $\alpha-\mathrm{ACTININ-stained} \mathrm{cardiomyocytes} \mathrm{were} \mathrm{captured} \mathrm{for} \mathrm{cell}$ surface measurement. The circumference of the stained cells was outlined manually and the cell surface area was automatically measured by using the Carl Zeiss AxioVision Rel 4.8 software.

\subsubsection{Analysis of sarcomere length and organization in hiPSC-CMs}

For analysis of sarcomere length and organization of the sarcomere structures, the images of $\alpha$-ACTININ-stained cardiomyocytes were analyzed using the Image $\mathbf{J}$ software as published previously (Weiwad et al., 2000).

Before the analysis, the pixels of the images were converted into $\mu \mathrm{m}$ depending on the microscope settings. For the images used in this experiment were taken with $63 \mathrm{x}$ oil lens with a spatial resolution of $0.102 \mu \mathrm{m} /$ pixel. The regular sarcomere region including at least 8 sarcomeres was chosen. After the chosen images were determined, the distance between the first and second peak was recorded, representing individual sarcomere lengths.

For analysis of sarcomeric organization, the observed striations aligned vertically in the field of view (FOV) were selected for further analysis. Two-dimensional fast Fourier transform (FFT) was processed on the selected region. Through FFT, the spatial domain of the intensity traces was transformed to the frequency domain (shown as radial profiles). Then the one-dimensional representation was calculated by plotting the radial profiles. The amplitude of the first peak represents the regularity of sarcomere structure in one direction, as the organization increases as more sarcomeric $\alpha$-ACTININ-positive elements are localized regularly at a distance of the sarcomere length. The full width at half 
maximum (FWHM) of the first-order line is a measure of the inhomogeneity of the sarcomere length (Figure 6).
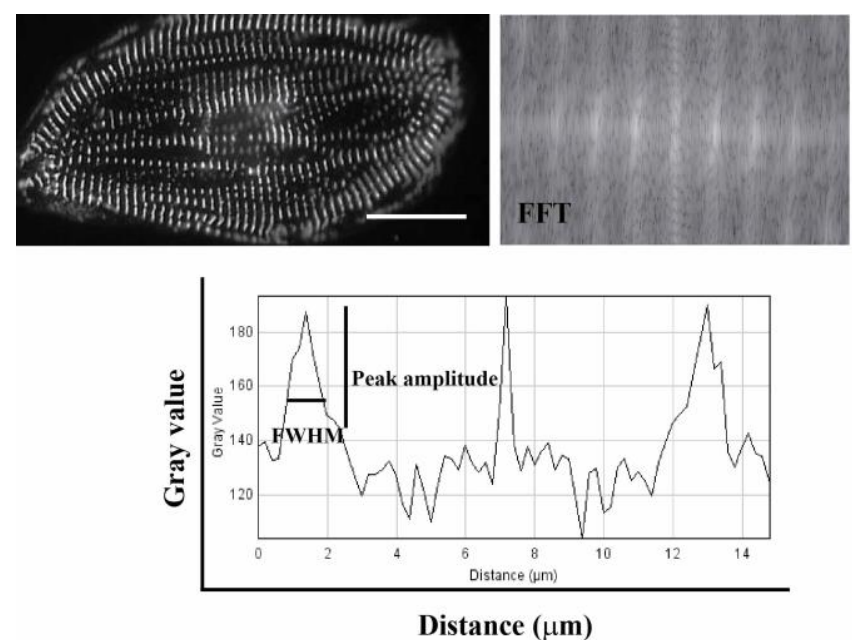

Figure 6. The scheme of quantitation of sarcomere structure. The sarcomere organization of $\alpha$-ACTININ stained cardiomyocytes was analyzed by FFT, scale bar: $20 \mu \mathrm{m}$.

\subsubsection{Flow cytometry and cell sorting}

Flow cytometry analysis was used to analyze the purity of cardiomyocytes by using BD FACSCantoII. The cells were digested with $0.25 \%$ trypsin into single cells and spun in a centrifuge. The resulting cell pellet was washed with DPBS twice and then fixed with $4 \%$ PFA for 20 min at room temperature. After washing 3 times with DPBS, the cells were blocked and permeabilized in 1\% BSA with $0.1 \%$ Triton X100 for $10 \mathrm{~min}$. The cells were then incubated with the primary antibody for $30 \mathrm{~min}$ at $37^{\circ} \mathrm{C}$. After removing the primary antibody by DPBS washing, fluorescence dye-coupled secondary antibody was applied and incubated for $30 \mathrm{~min}$ at $37^{\circ} \mathrm{C}$. After washing with DPBS, the cell pellets were spun down and suspended in $200 \mu \mathrm{LPBS}$ and transferred into the 5-ml FACS tube. For flow cytometer analysis, the 10,000 total events were included. The results were analyzed by Flowing software.

For cell sorting, hiPSCs that were transfected with CRISPR/Cas9 plasmid DNA were digested with TrypLE Express for $5 \mathrm{~min}$ at room temperature. The cell pellets were first sorted using a $25-\mu \mathrm{m}$ cell strainer, suspended in DMEM/F12 without phenol red and transferred into the 5-ml FACS tube. Single hiPSC was then sorted into the Geltrex-coated 96-well plates in E-8 medium using BD FACSAria II. 


\subsubsection{Cell volume analysis}

The cell volumes of cardiomyocytes were analyzed during flow cytometry analysis. Flow cytometry analysis was performed as described before and further analysis of forward-scattered light (FSC), which represents the cell volume of digested cardiomyocytes, was performed with the Flowing software (Figure 7).

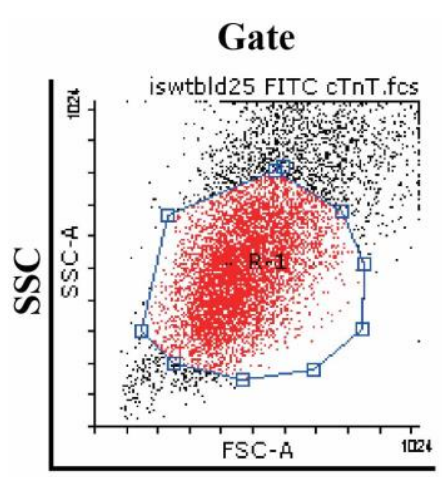

FSC

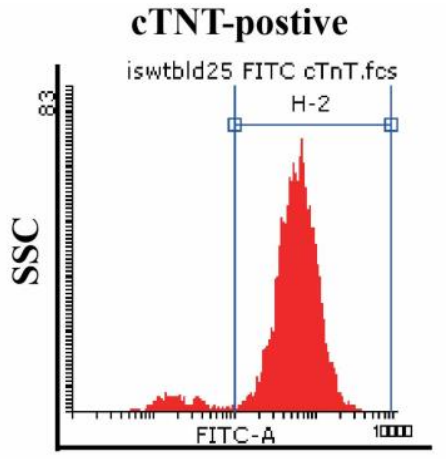

cTNT

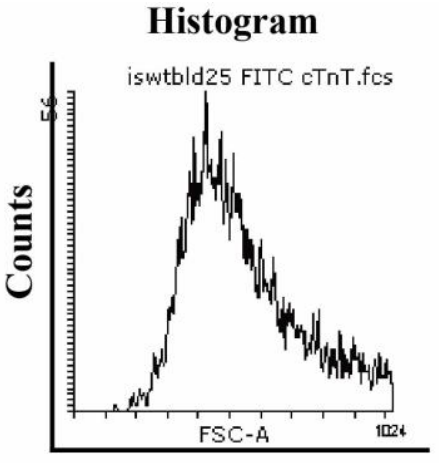

FSC

Figure 7. Analysis of cardiomyocyte volume using flow cytometry. The cTNT-positive cardiomyocytes were analyzed with the FSC.

\subsubsection{Cleavage assay}

To determine the cleavage efficacy of the designed sgRNA, HEK293T cells were first transfected with CRISPR/Cas9-GFP plasmid DNA. Briefly, the HEK 293T cells were passaged and cultured until $60 \%$ confluence before transfection. The cells were transfected with CRISPR/Cas9-GFP for $48 \mathrm{~h}$ according to the manufacturer's instructions of TransIT-293 reagent. Genomic DNA was then extracted from the transfected and non-transfected cells. PCR was performed using the EPG5_3 primer set (2.1.1) according to the protocol provided in the genomic cleavage detection kit. The positive control provided in the kit was included. The PCR product was then denatured and re-annealed to produce heteroduplex mismatches. These mismatches were recognized and cut by the detection enzyme provided in the kit. The cleavage PCR product was detected and quantified using $1 \%$ agarose gel. 


\subsubsection{Engineered heart muscle}

\subsubsection{Generation of engineered heart muscle}

In this study, EHMs were generated based on the published protocol (Soong et al., 2012). Briefly, hiPSC-derived cardiomyocyte cultures were dissociated into single cardiomyocytes using a mixture of $2.5 \%$ trypsin $(0.25 \%)$, accutase $(97.75 \%)$ and $1 \mathrm{mg} / \mathrm{ml}$ DNAase (2\%). Non-myocytes that provide tissue support such as human foreskin fibroblasts were also concurrently dissociated with TrypLE Express at $37^{\circ} \mathrm{C}$ for $3 \mathrm{~min}$. Together, the cell mixture containing single cardiomyocytes and fibroblasts with a ratio of 3:1 were mixed with the reagents as listed in the table below. $450 \mu \mathrm{l}$ of the total master mix was transferred on individual casting mold recesses and incubated at $37^{\circ} \mathrm{C}$ for $1 \mathrm{~h}$. Complete medium was then layered over the EHMs and allowed to incubate till tissue compaction. Culture medium was exchanged daily. After $72 \mathrm{~h}$, the EHMs were manually transferred onto silicone poles to induce mechanical stretch. Medium was changed every 2 days until day 14. The EHMs were then removed from the poles and subjected to force measurements in thermostatted organ baths.

\begin{tabular}{ll}
\hline Total volume & $\mathbf{2 1 0 0} \boldsymbol{\mu l}$ \\
\hline Cell suspension $\left(1.5 \times 10^{6}\right.$ per EHM) & $829 \mu \mathrm{l}$ \\
\hline $\mathbf{2 x}$ DMEM & $535 \mu \mathrm{l}$ \\
\hline NaOH 0.1 N & $95 \mu \mathrm{l}$ \\
\hline Matrigel & $200 \mu \mathrm{l}$ \\
\hline Rat collagen $(3.2 \mathbf{~ m g / m l})$ & $440 \mu \mathrm{l}$ \\
\hline Number of EHMs & 4 \\
\hline
\end{tabular}

\subsubsection{Force measurement of engineered heart muscle}

Force measurement studies were carried out in thermostatted organ baths. Briefly, EHMs were carefully removed from the poles and placed onto the attached hooks on the force transducer. The organ baths were then filled with Tyrode buffer at $37^{\circ} \mathrm{C}$. EHMs were then field stimulated at $2 \mathrm{~Hz}$ and manually stretched to allow for maximum force production. Thereafter, EHMs were subjected to increasing calcium concentration $(0.2-4 \mathrm{mM})$ to 
determine their calcium response and twitch forces were recorded using BMON software (FMI GmBH, Germany).

\subsubsection{Cell size measurement of CMs in EHMs}

Before the generation of EHMs, the diameters of cardiomyocytes were analyzed by using the Casy cell counter. Briefly, cardiomyocytes were dissociated into single cells as mentioned before. After the hEHM medium was added to neutralize trypsin, the cell pellets were spun down at $200 \times \mathrm{g}$ for $5 \mathrm{~min}$. The pellets were re-suspended in $1 \mathrm{ml}$ hEHM medium. According to the manufacturer's instructions, $1 \mu$ l cell suspension was diluted 20 times with $\mathrm{H}_{2} \mathrm{O}$. Finally, based on the non-invasive electrical current exclusion principle, the diameters of the cardiomyocytes were automatically analyzed by using the Casy cell counter.

\subsection{Statistics}

All experimental data is presented as mean \pm standard error of the mean (SEM). For two data sets, unpaired Student's $t$-test was used. For more than two variables, analysis was performed using a two-way repeated analysis of variance (ANOVA) test. Statistically significance is presented as $\mathrm{p}<0.05(*), \mathrm{p}<0.01(* *)$ and $\mathrm{p}<0.001(* * *)$. All statistical analysis was carried out by using GraphPad Prism software (Version 6.01). 


\section{Results}

\subsection{Generation of Vici-iPSCs and proof of pluripotency}

\subsubsection{Generation of hiPSCs from the patient with Vici syndrome}

For the generation of Vici-iPSCs, fibroblasts derived from the patient with Vici syndrome were reprogrammed into hiPSCs through STEMCCA lentivirus transduction, which contains the transcription factors $O C T 4, S O X 2, K L F 4$ and $c-M Y C$ in a single polycistronic vector. Because the proliferation rate of fibroblasts derived from this patient was quite slow, $6 \times 10^{4}$ fibroblasts at passage 3 in a well of a 12-well plate were transduced with STEMCCA virus for $24 \mathrm{~h}$ in the presence of $1 \mu \mathrm{g} / \mathrm{ml}$ polybrene (Figure 8). The transduced fibroblasts were cultured in HFBM with $10 \mathrm{ng} / \mathrm{ml}$ bFGF for 7 days and then passaged into Geltrex-coated dishes. E-8 medium with additional $10 \mathrm{ng} / \mathrm{ml}$ bFGF was applied to the culture $24 \mathrm{~h}$ later and used until iPSC colonies were observed. Colonies with the typical morphology of human pluripotent stem cells (tightly packed, sharp-edged, flat and high nuclear-cytoplasmic ratio) were picked at 30-40 days after transduction and further expended to establish the stable cell lines.

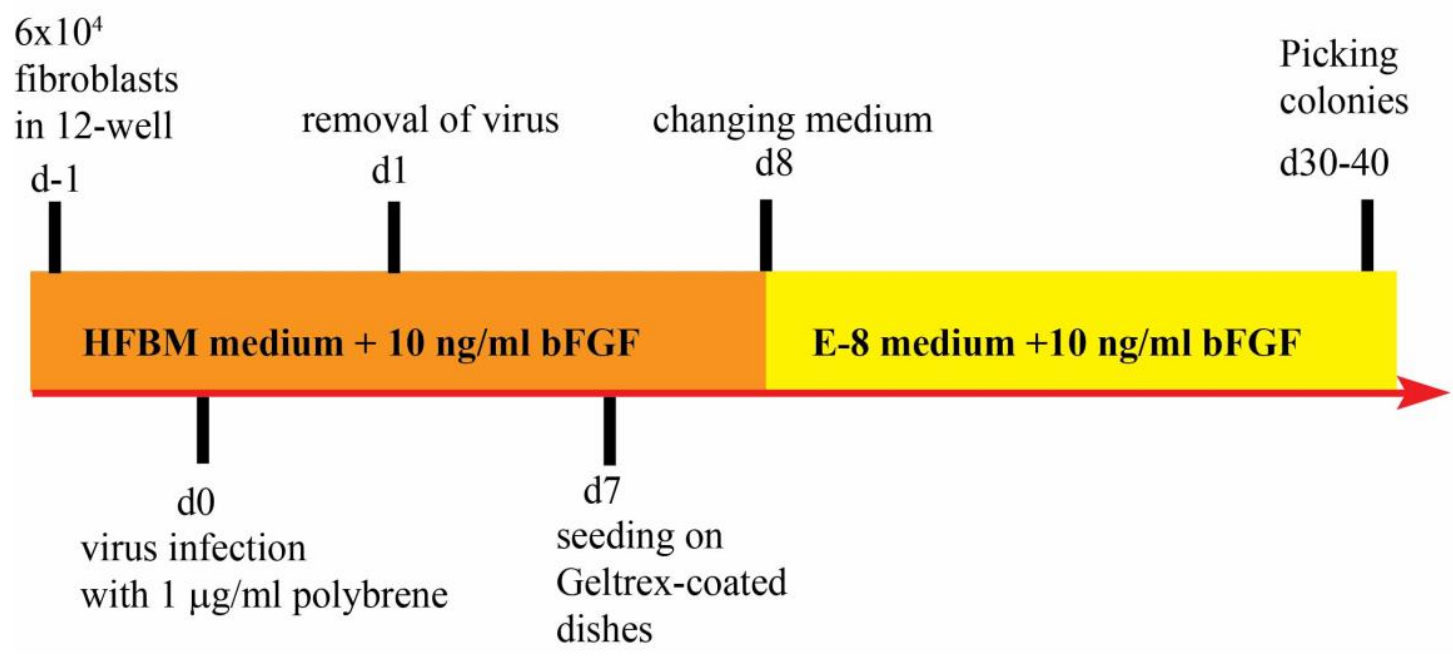

Figure 8. The scheme of iPSC generation from fibroblasts derived from the patient with Vici syndrome.

In total, five independent iPSC lines were established and three of them, named as iVici1.2, iVici1.3 and iVici1.5, were used for further pluripotency characterization. They were cultured on Geltrex-coated dishes in E-8 medium and showed typical morphology of human pluripotent stem cells (Figure 9A-C). Alkaline phosphatase staining as the first marker of successful reprogramming was performed at passage 6 . The results showed that 
the three analyzed iPSC lines were positive for alkaline phosphatase (Figure 9D-F).
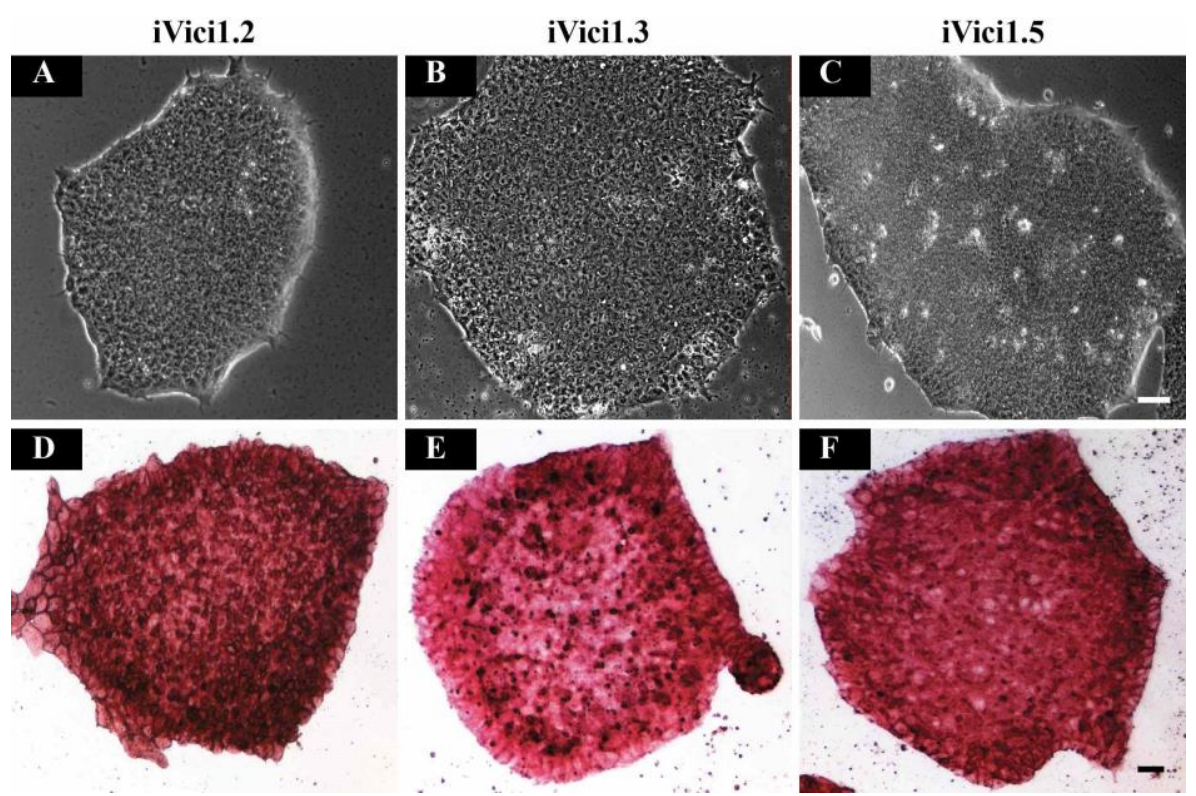

Figure 9. Alkaline phosphatase staining in Vici-iPSCs. The established Vici-iPSCs at passage 6 (A-C) were positive for alkaline phosphatase activity (D-E). Scale bar: $50 \mu \mathrm{m}$.

\subsubsection{Expression of pluripotent-related markers in Vici-iPSCs}

To investigate the pluripotency of Vici-iPSCs, pluripotent-related genes were analyzed by semi-quantitative RT-PCR (Figure 10). During cellular reprogramming, endogenous pluripotent-related genes were upregulated. The results showed that GDF3, FOXD3, LIN28 and SOX2 were highly expressed in Vici-iPSCs, which were comparable to those in hESCs.

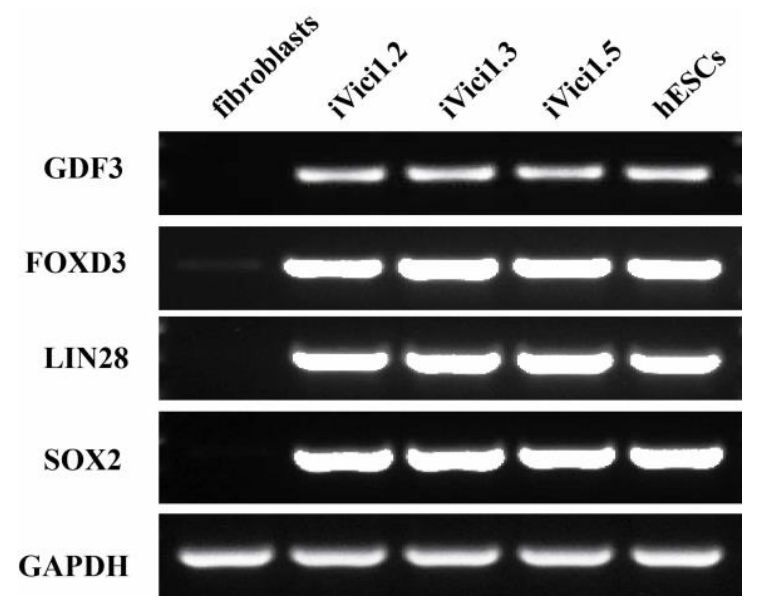

Figure 10. Expression of pluripotent-related genes in Vici-iPSCs. The pluripotent genes GDF3, FOXD3, LIN28 and SOX2 were expressed in the three analyzed Vici-iPSC lines. The fibroblasts derived from the patient were used as negative control whereas the RNA isolated from human embryonic stem cells (hESCs) was used as positive control. 
Expression of the pluripotent-related markers was also confirmed in all analyzed Vici-iPSC lines by immunofluorescence staining with primary antibodies against OCT4, SOX2, NANOG, LIN28 and SSEA4 (Figure 11). Transcription factors OCT4, SOX2 and NANOG were located in the nuclei. LIN28, a RNA-binding protein, which acts as a posttranscriptional regulator of genes involved in developmental timing and self-renewal of stem cells, was located in the cytoplasm. SSEA4 as an early embryonic glycolipid antigen was located on the cell surface.
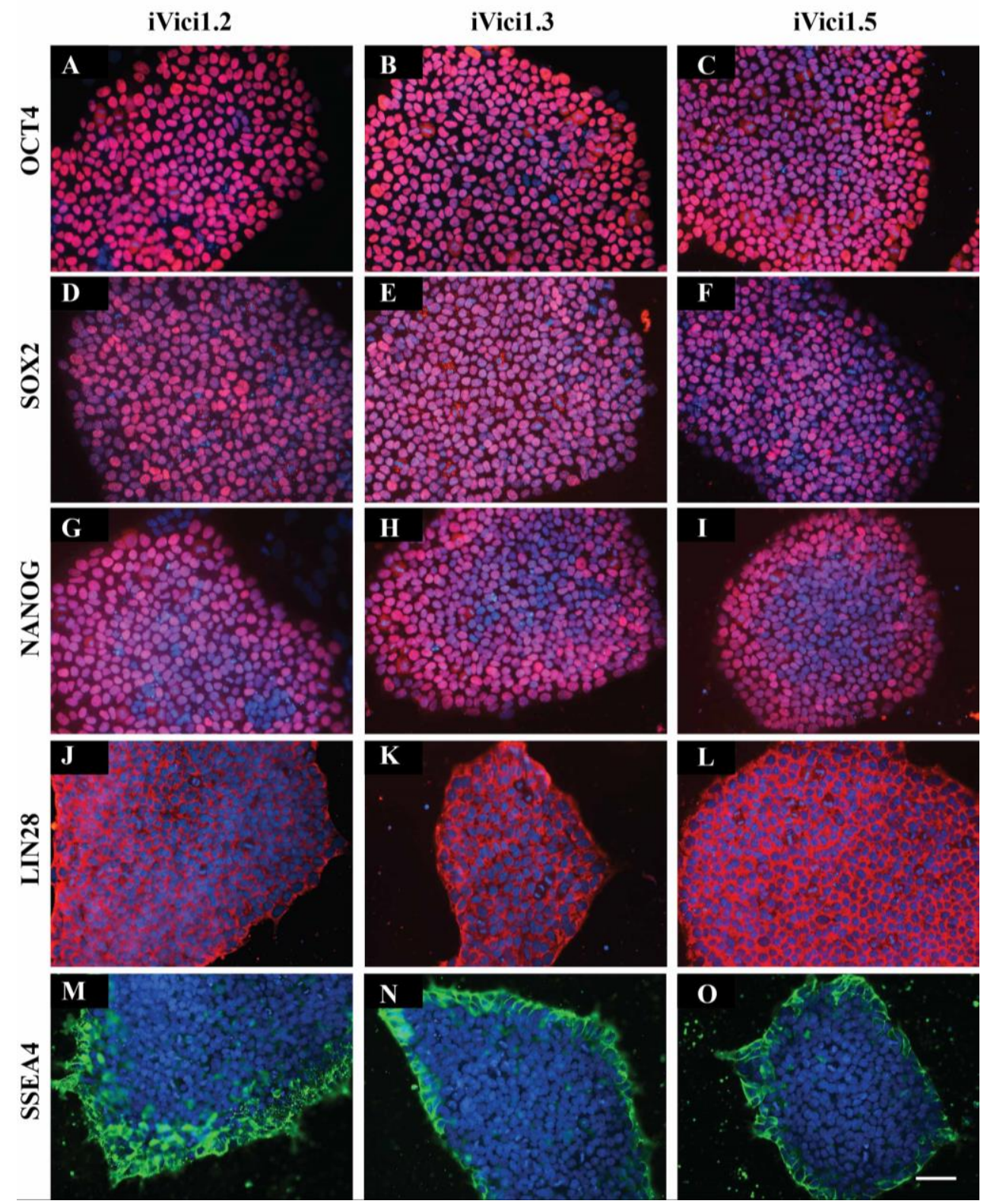

Figure 11. Expression of pluripotent-related proteins in Vici-iPSCs. All three analyzed Vici-iPSC lines highly expressed pluripotent markers OCT4 (A-C, red), SOX2 (D-F, red), NANOG (G-I, red), LIN28 (J-L, red) and SSEA4 (M-O, green). Cell nucleus was stained with DAPI (blue). Scale bar: $50 \mu \mathrm{m}$. 


\subsubsection{Spontaneous differentiation of Vici-iPSCs in vitro and in vivo}

Pluripotency of Vici-iPSCs was assessed by both in vitro and in vivo spontaneous differentiation via EB formation and teratoma generation, respectively. For in vitro differentiation, Vici-iPSCs were cultivated in suspension for 8 days in Iscove medium to form three-dimensional cell aggregates, so called EBs, which were then plated onto gelatin-coated dishes for further differentiation until day 33. Upon the formation of EBs, all analyzed Vici-iPSC lines started to differentiate spontaneously. Cells representing all three germ layers were found in EB outgrowths at day 33 as demonstrated by the positive staining by using antibodies against AFP suggesting endodermal development, SMA indicating mesodermal development and $\beta$-III-TUBULIN, a marker for neuroectodermal development (Figure 12).
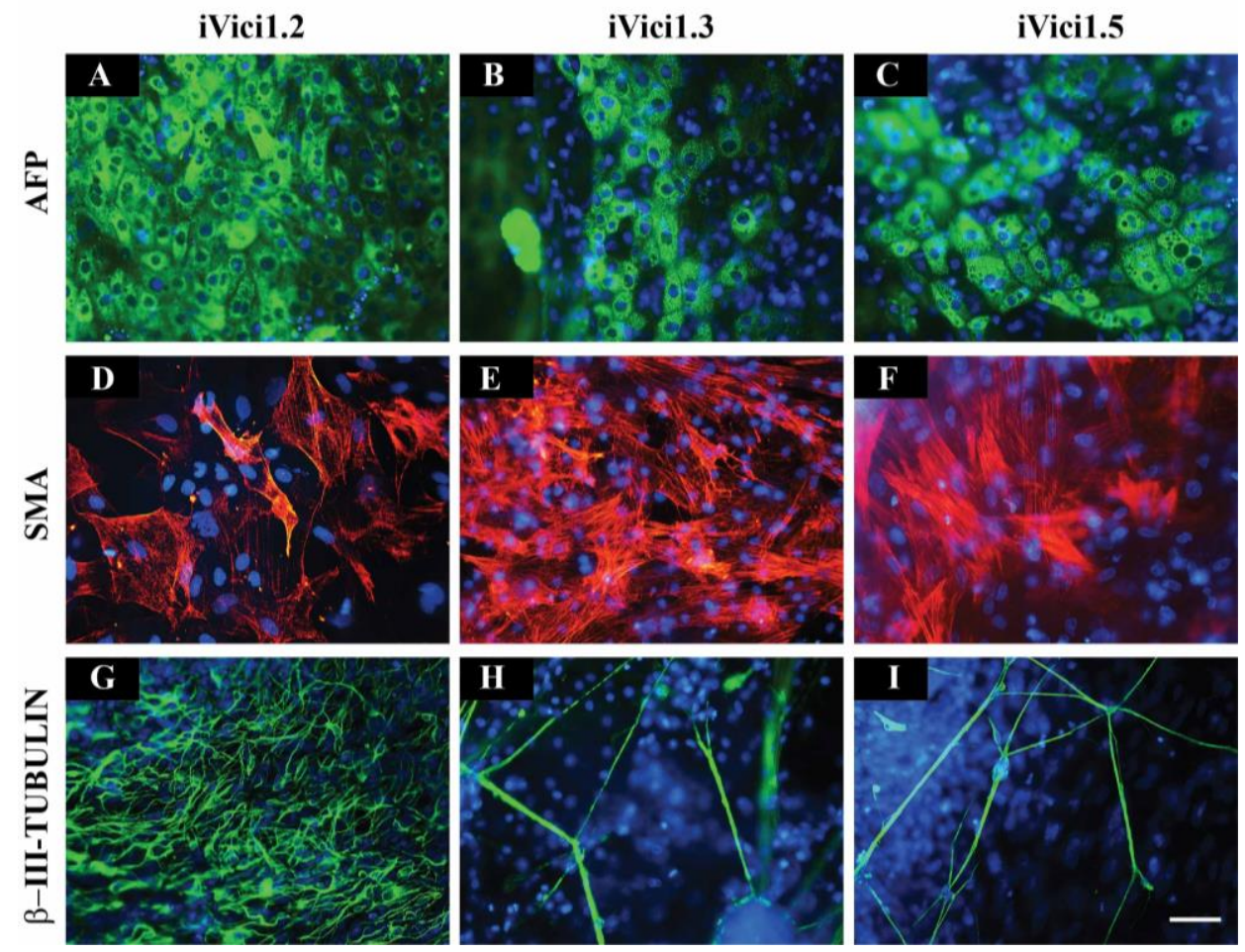

Figure 12. Spontaneous differentiation of Vici-iPSCs in vitro. Vici-iPSCs spontaneously differentiated into cells from all three germ layers via EB formation. Immunofluorescence staining results showed that the representative markers of endoderm AFP (A-C, green), mesoderm SMA (D-F, red) and neuroectoderm $\beta$-III-TUBULIN (G-I, green) were expressed in cells differentiated from iVici1.2, iVici1.3 and iVici1.5 cells. Cell nucleus was stained with DAPI (blue). Scale bar: $50 \mu \mathrm{m}$.

To study the in vivo teratoma development potential of the iPSCs, Vici-iPSCs $\left(9 \times 10^{6}\right.$ cells per mouse) were subcutaneously injected into immunodeficient mice ( $\mathrm{n}=2$ for each cell line). Only iVici1.3 cells showed teratoma formation at day 59 after inoculation of the cells. The teratomas were dissected and processed with standard H\&E staining. Histological 
analysis of the sections showed that the teratomas were composed of derivatives of three embryonic germ layers (Figure 13).
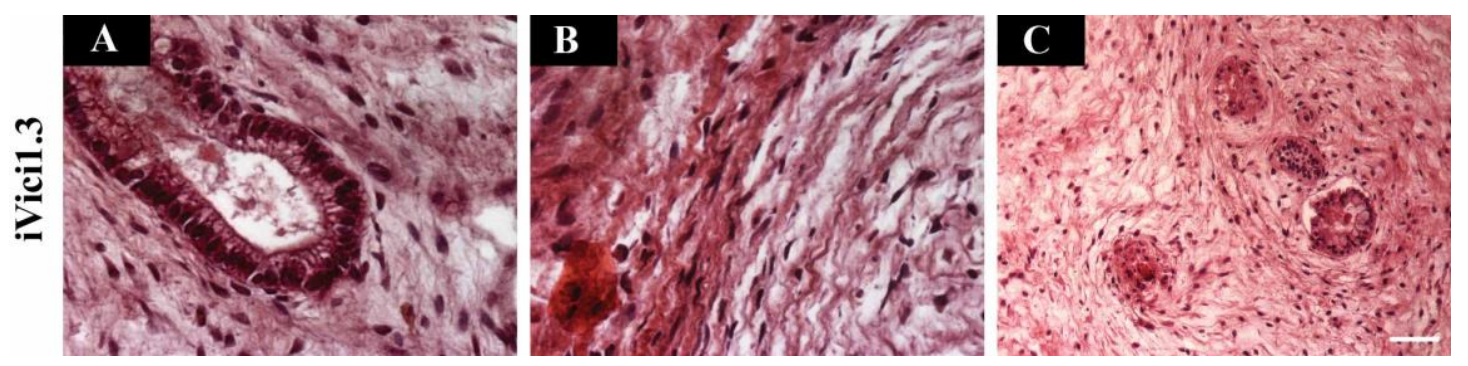

Figure 13. H\&E staining of teratoma generated from iVici1.3 cells in vivo. The teratoma contained derivatives of three embryonic germ layers: epithelium (endoderm, A), muscle structure (mesoderm, B) and neural-like tissue (ectoderm, C). Scale bar: $50 \mu \mathrm{m}$.

\subsubsection{Verification of EPG5 mutation in Vici-iPSCs}

Previous study showed that the patient with Vici syndrome carried a single nucleotide mutation site in EPG5 (c. 4952+1G>A) (Cullup et al., 2013), from whom the three Vici-iPSC lines iVici1.2, iVici1.3 and iVici1.5 were generated. To prove whether the mutation was kept in these three Vici-iPSC lines, genomic DNA sequencing was performed using the primer set EPG5_2 as shown in Figure 14A as F1 and R1. The sequencing results confirmed that the homogeneous mutation site $(c .4952+1 \mathrm{G}>\mathrm{A})$ was retained in the three Vici-iPSC lines after cellular reprogramming (Figure 14B).

A

Genomic DNA

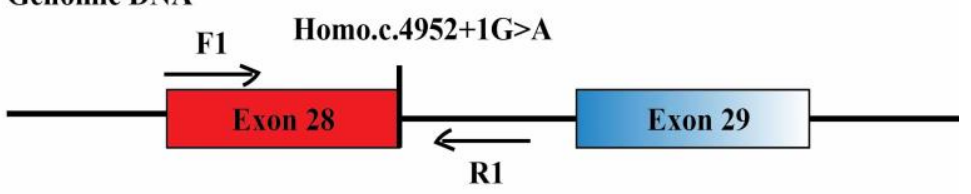

B

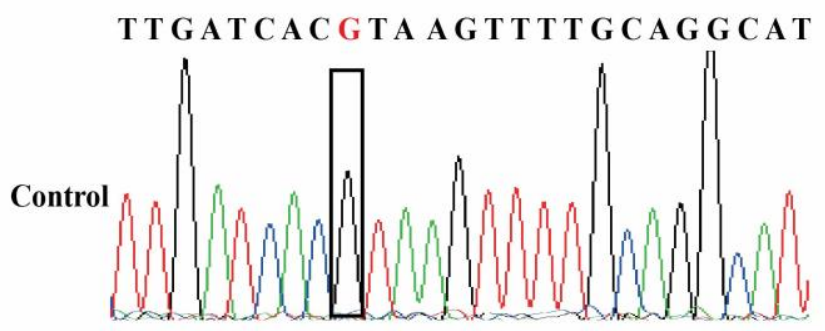

T T GAT CACATA A G T T T T G C A G G CA T

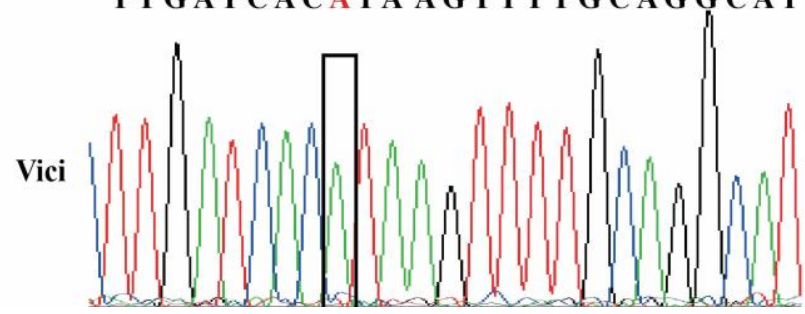

Figure 14. Verification of the EPG5 mutation in the genome of Vici-iPSCs.

Primers F1 and R1 were used to amplify the region containing the mutation site in genomic DNA (A). The EPG5 mutation (c.4952+1G >A) retained in Vici-iPSCs after cellular reprogramming was confirmed by sequencing $(\mathbf{B})$. 
The mutation in EPG5 (c.4952+1G>A) occurs within the splice site between exon 28 and 29 (Figure 14A), which may disrupt the mRNA splicing. Exome sequencing predicted that this mutation in EPG5 caused the exon 28 missing (Cullup et al., 2013) and resulted in a premature stop codon in exon 29. Further analysis on the cDNA level with the primer set EPG5_1 (2.1.1) as shown in Figure 15A as F2 and R2 showed that EPG5 mRNA transcript was 100 bp nucleotides shorter in Vici-iPSCs comparing with the Ctr-iPSCs (Figure 15 B).

A

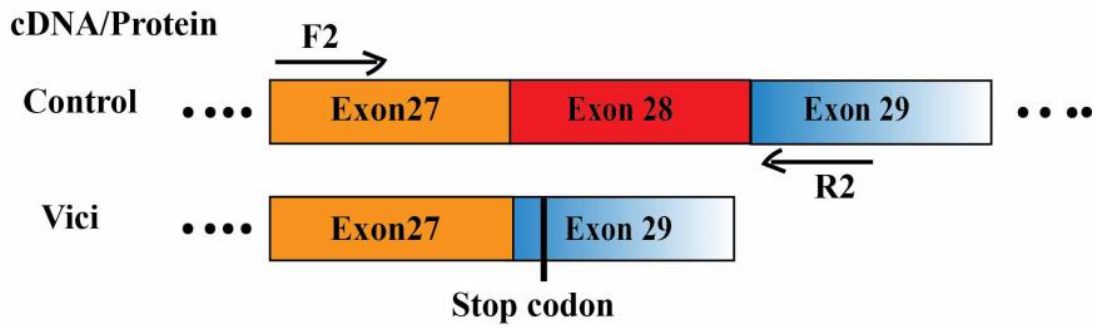

B

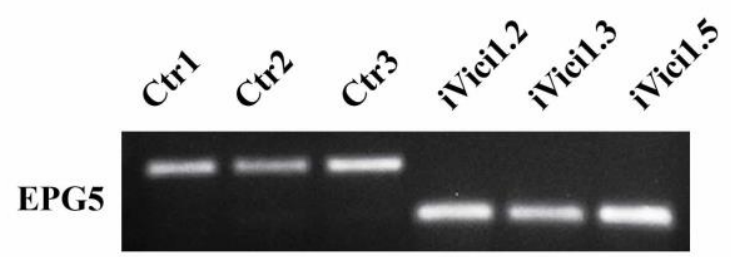

Figure 15. Verification of EPG5 mutation on mRNA level in Vici-iPSCs. The shorter product of EPG5 on mRNA level was seen in Vici-iPSCs compared with Ctr-iPSCs by RT-PCR (B) using the primer set F2 and R2 (A).

Taken together, all three analyzed Vici-iPSC lines iVici1.2, iVici1.3 and iVici1.5 showed the typical morphology of human pluripotent stem cells, were positive for ALP, expressed pluripotent-related markers demonstrated by the RT-PCR analysis and immunofluorescence staining, and exhibited in vitro and in vivo differentiation potential. These data demonstrate that the generated and analyzed Vici-iPSC lines are pluripotent. Furthermore, the EPG5 mutation was verified in all three analyzed Vici-iPSC lines.

\subsection{Generation and phenotype analysis of Vici-iPSCs-derived cardiomyocytes}

\subsubsection{Differentiation of cardiomyocytes from Ctr-iPSCs and Vici-iPSCs}

Cardiomyocytes derived from the iPSCs were generated through modifying the Wnt pathway by the GSK3 $\beta$ inhibitor CHIR99021 and the Wnt inhibitor IWP2 (Figure 16). 
After 1 day of CHIR99021 exposure, hiPSCs differentiated into mesendoderm progenitor cells, which have the capacity to develop into muscle cells. After further 2 days of IWP2 exposure, these mesodermal progenitor cells became committed towards cardiomyocyte specification. First beating cardiomyocytes were observed between day 8 and 12 . As cell seeding densities and concentration of CHIR99021 directly influenced the yield of cardiomyocytes from the differentiation, the protocol was adapted and optimized to different iPSC lines. The control iPSC lines isWT1BLD2 (Ctr1), WTD2 (Ctr2), iBM76.3 (Ctr3) and Vici-iPSC lines iVici1.2, iVici1.3, and iVici1.5 were differentiated into functional cardiomyocytes. Subsequent experiments mostly used the Ctr1, 2 and iVici1.2 and iVici1.3 derived cardiomyocytes following culture for 2-3 months.

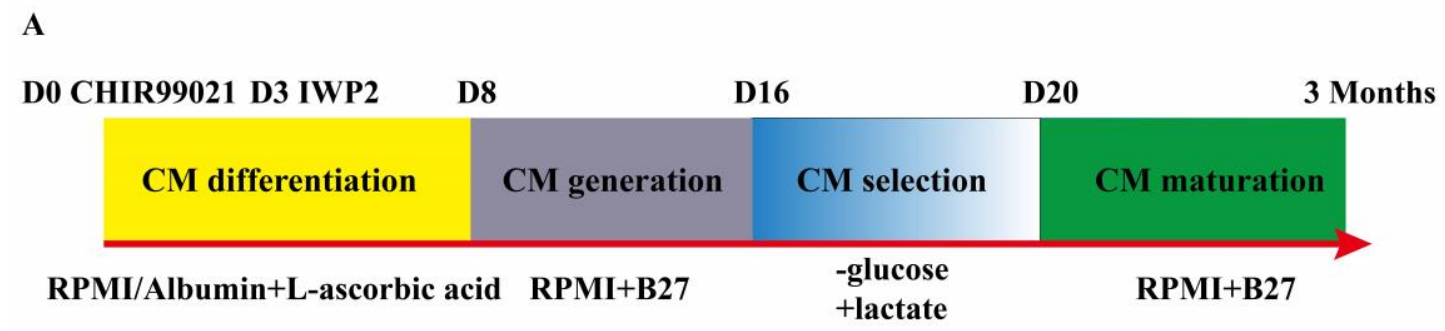

B
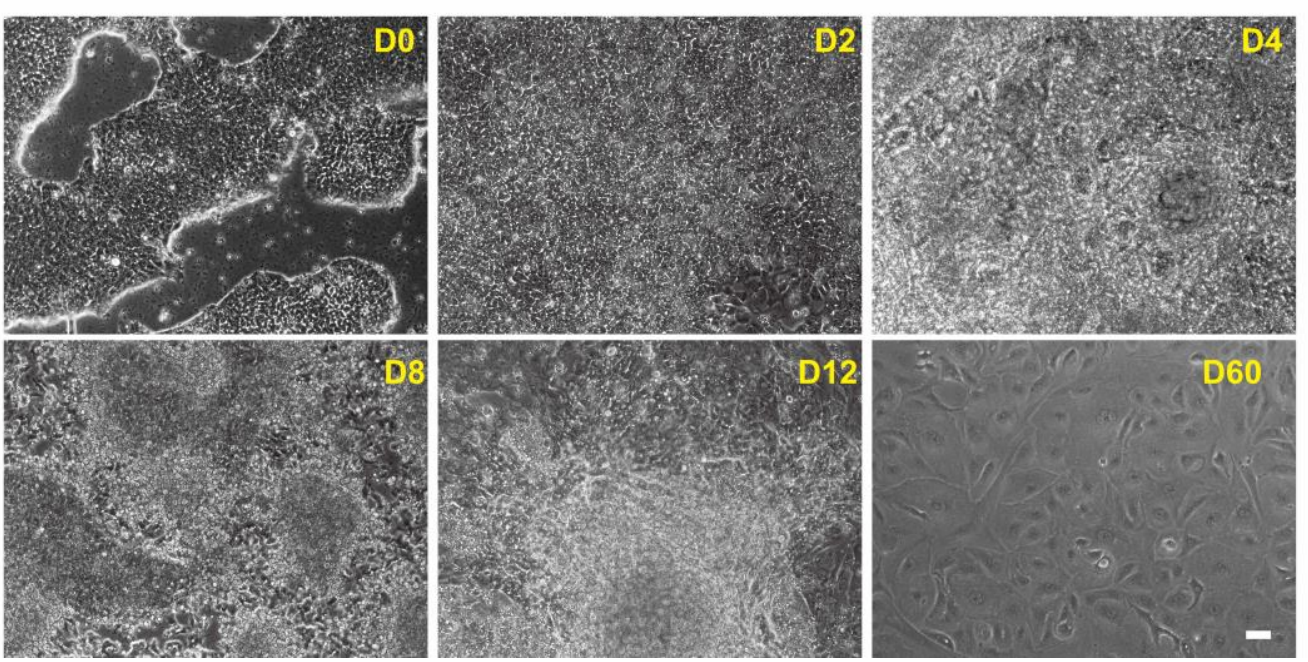

Figure 16. Direct differentiation of hiPSCs into cardiomyocytes. The cardiomyocytes were differentiated according to the modified protocol (A). Brightfield images were captured and showed the typical density and morphology of hiPSCs during the differentiation on day $0,2,4,8$, 12 and 60 (B). Scale bar: $50 \mu \mathrm{m}$.

In order to obtain pure population of cardiomyocytes, glucose was withdrawn from the differentiation medium and replaced by lactate to select metabolically active cardiomyocytes while reducing the population of non-cardiomyocytes. The cells were selected for 4-6 days. To determine the purity of cardiomyocytes following differentiation and selection, cells were harvested and incubated with antibody against cTNT. Results 
from flow cytometry showed high purity of cardiomyocytes (appr. 90\%), suggesting the differentiation and selection method is efficient in yielding pure cardiomyocytes (Figure 17). The differentiation efficiency and CM purities of different Ctr- and Vici-iPSC lines showed no significant difference (Figure 17).

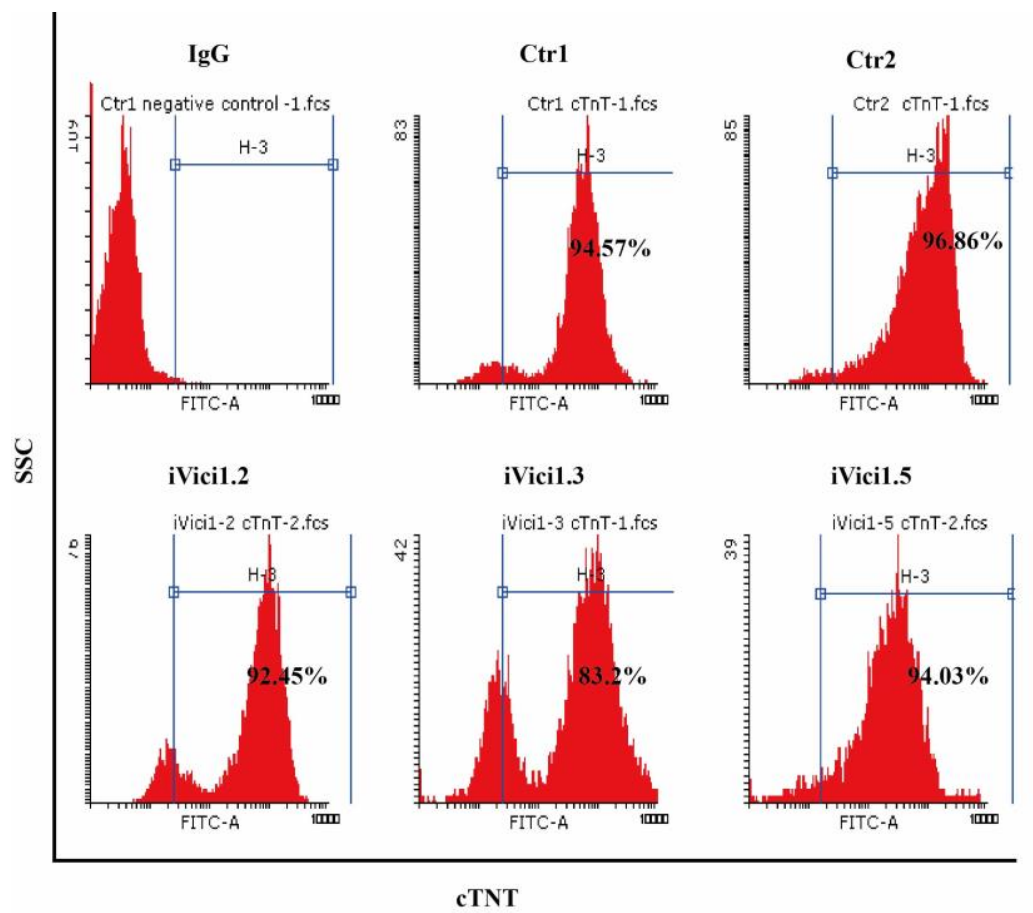

Figure 17. Efficiency of direct differentiation of hiPSCs into cardiomyocytes. Flow cytometry analysis of cTNT-positive cells was performed after cultivation in cardiac culture medium (RPMI+B27) for 2-3 months. The results showed the efficiency of cardiac differentiation from Ctr-iPSCs and Vici-iPSCs. Ctr1: 94.57\%; Ctr2: 96.86\%; iVici1.2: 92.45\%; iVici1.3: 83.2\% and iVici1.5: $94.03 \%$.

Additionally, to confirm our observation of cardiomyogenesis from the differentiation protocol, gene expression of cardiac-specific markers using RT-PCR was investigated. The results showed that $M Y H 7, M Y L 2$, and genes encoding cardiac transcriptional factor NKX2.5 and sarcomeric structural components TNNT2 were highly expressed in cardiomyocytes derived from both Ctr-iPSCs and Vici-iPSCs compared to undifferentiated hiPSCs. There was no significant difference observed among different Ctr- and Vici-iPSC lines (Figure 18). 


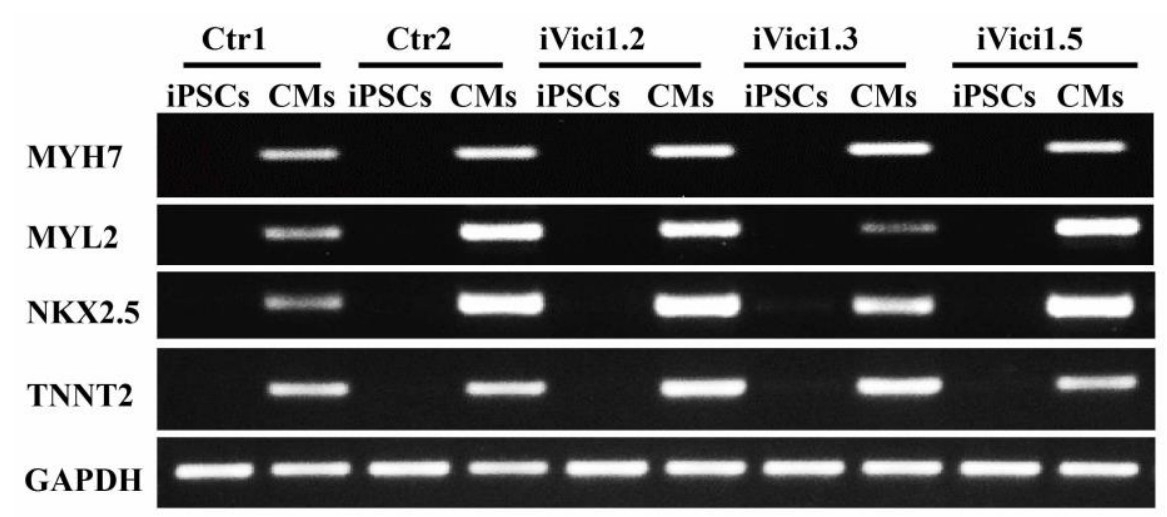

Figure 18. Gene expression of cardiac-related markers by RT-PCR. The RT-PCR analysis of cardiac-related genes MYH7, MYL2, NKX2.5 and TNNT2 was performed. These genes were highly expressed in both Ctr-iPSCs- and Vici-iPSCs-derived cardiomyocytes compared with undifferentiated iPSCs.

Next, protein expression of cardiac-specific markers was analyzed by immunofluorescence staining. The results showed that cardiomyocytes derived from both Ctr- and Vici-iPSC lines expressed myofilament proteins such as cTNT, $\alpha$-ACTININ and MLC2V. The gap junction protein CX43 was highly expressed and detected between adjacent cardiomyocytes derived from both Ctr- and Vici-iPSC lines (Figure 19).
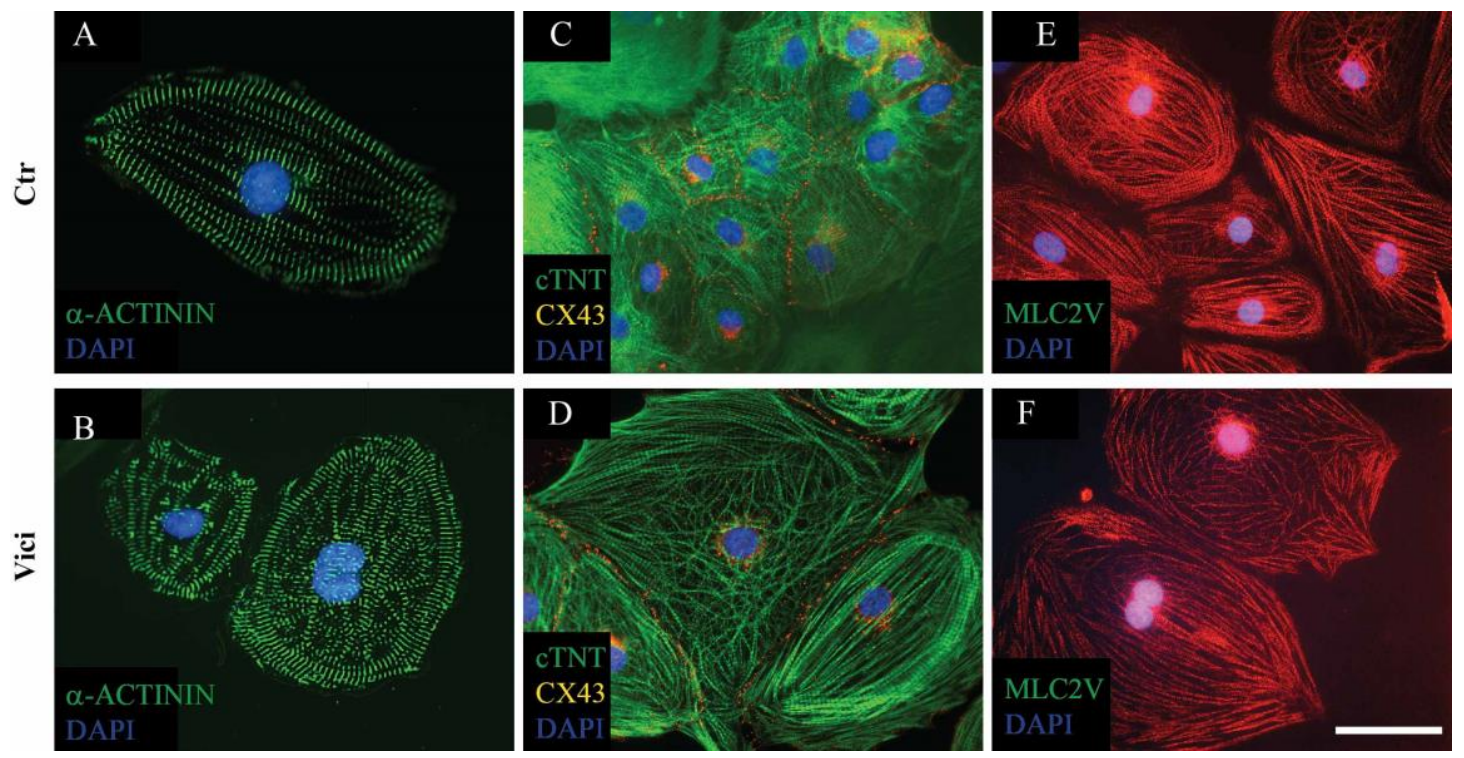

Figure 19. Structural characterization of Ctr- and Vici-iPSC-CMs. The cardiomyocytes were singularized and plated onto the Geltrex-coated coverslips. Immunofluorescence staining of $\alpha$-ACTININ (A and B, green), cTNT (C and D, green) and MLC2V (E and F, red) showed the sarcomere organization. The gap junction protein CX43 was detected between adjacent cells (C and $\mathbf{D}$, red). Cell nucleus was stained with DAPI (blue). Scale bar: $50 \mu \mathrm{m}$.

Altogether, purified cardiomyocytes that highly expressed the cardiac-related markers 
including $\alpha$-ACTININ, MLC2V and cTNT can be obtained from both Ctr- and Vici-iPSCs with high efficiency using the differentiation protocol mentioned in this study. These functional beating cardiomyocytes were subsequently used for further phenotype analysis.

\subsubsection{Vici-iPSCs-CMs show larger cell surface area but normal cell volume}

In order to investigate if Vici-iPSC-CMs carry the hypertrophy phenotype in vitro, the cell surface area of both Ctr- and Vici-iPSC-CMs was measured. The cardiomyocytes were singularized after culturing for 2-3 months and stained with antibodies targeting sarcomeric $\alpha$-ACTININ. Images of randomly selected single cardiomyocytes were captured and surface area was analyzed using AxioVision software based on the immunofluorescence staining (Figure 20A, B). The results showed that the average cell surface area of Vici-iPSC-CMs (Vici Nc: $4564 \pm 692.8 \mu \mathrm{m}^{2}$ ) was significantly larger compared to Ctr-iPSC-CMs (Ctr Nc: $2226 \pm 127.8 \mu \mathrm{m}^{2}$ ) under basal conditions (Figure 20C).

To rule out autophagy-induced hypertrophy of Vici-iPSC-CMs, cardiomyocytes were treated with the autophagy inducer rapamycin and blocker bafilomycin. Interestingly, despite DMSO, rapamycin and bafilomycin treatments, the cell surface areas of Vici-iPSC-CMs remain significantly larger than that of Ctr-iPSC-CMs (Figure 20C). There was no big difference among untreated (Nc), DMSO and drug treatment groups in either Ctr- or Vici-iPSC-CMs. 

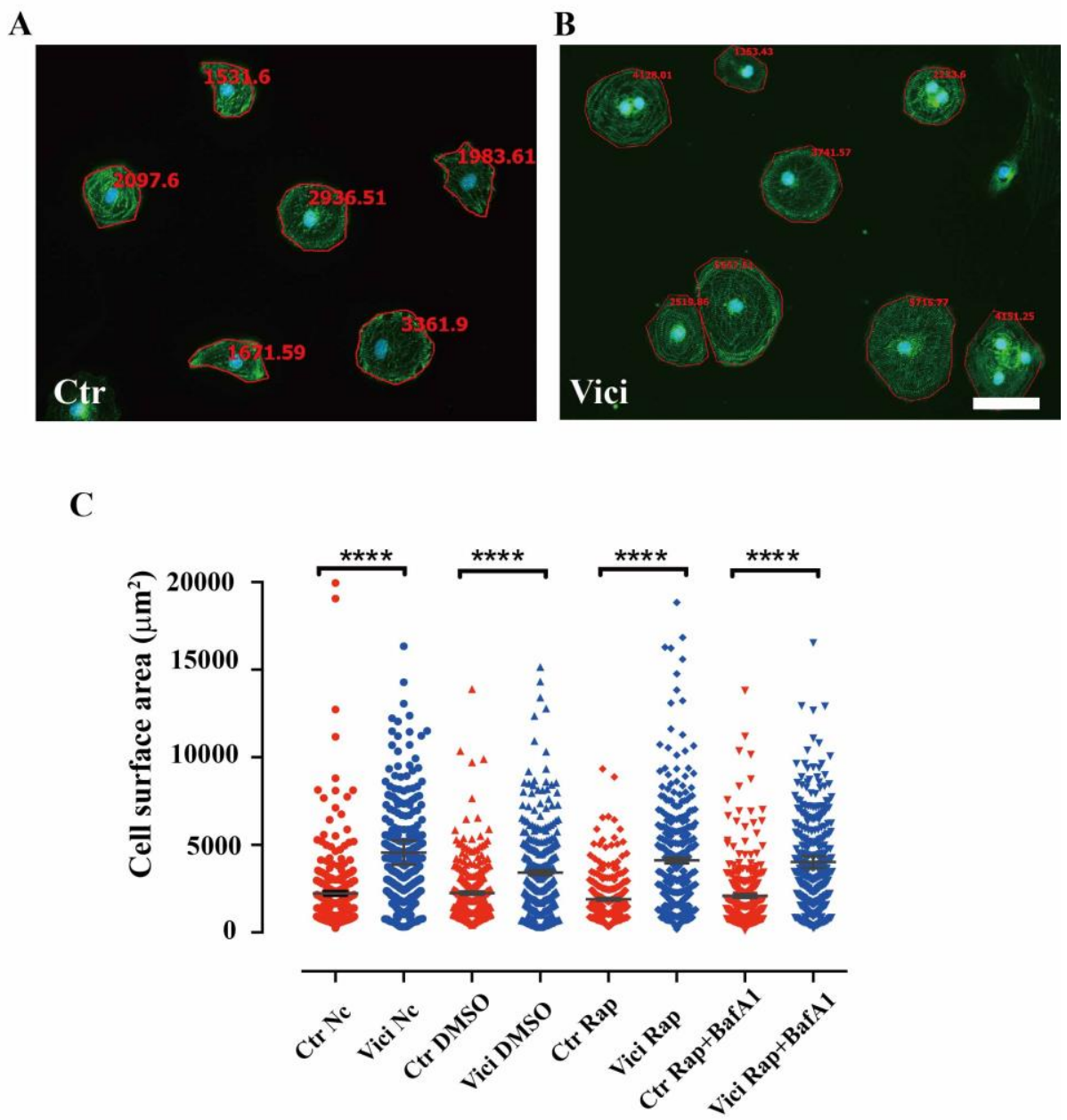

Figure 20. Measurement of cell surface area of Ctr- and Vici-iPSC-CMs. The cell surface area was measured in CMs 10 days post dissociation after 3-month culture (A and $\mathbf{B}$ ). The average cell surface area was bigger in Vici-iPSC-CMs compared with Ctr-iPSC-CMs under basal condition, DMSO, rapamycin and rapamycin with bafilomycin treatment $(\mathbf{C})$. In total, the iPSC lines from control (Ctr1 and Ctr2) and Vici syndrome (iVici1.2, iVici1.3 and iVici1.5) were used. The mean area with SEM and cell number from each group is illustrated in the following: Ctr Nc: $2226 \pm 128$ (n=299); Ctr DMSO: $2245 \pm 96$ (n=302); Ctr Rap: $1903 \pm 77$ (n=301); Ctr Rap+BafA1: $2085 \pm 106$ ( $\mathrm{n}=299)$; Vici Nc: $4564 \pm 693$ ( $\mathrm{n}=381)$; Vici DMSO: $3409 \pm 147$ $(n=338)$; Vici Rap: $4112 \pm 159$ ( $n=358)$; Vici Rap+BafA1: $4023 \pm 339$ ( $n=368)$; unit, $\mu m^{2}$. Scale bar: $50 \mu \mathrm{m}$.

Moreover, the volumes of cardiomyocytes were analyzed by flow cytometry analysis. Both Ctr- and Vici-iPSC-CMs were stained with antibody against the sarcomeric structural protein cTNT. The overlap histogram of FSC of the cTNT-positive cells demonstrated that Vici-iPSC-CMs had similar cell volume to Ctr-iPSC-CMs (Figure 21). 
A

Ctr1

B

Ctr2

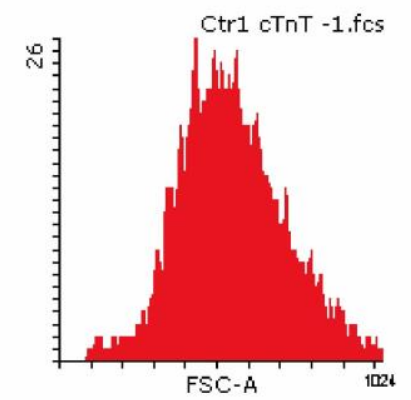

C

D
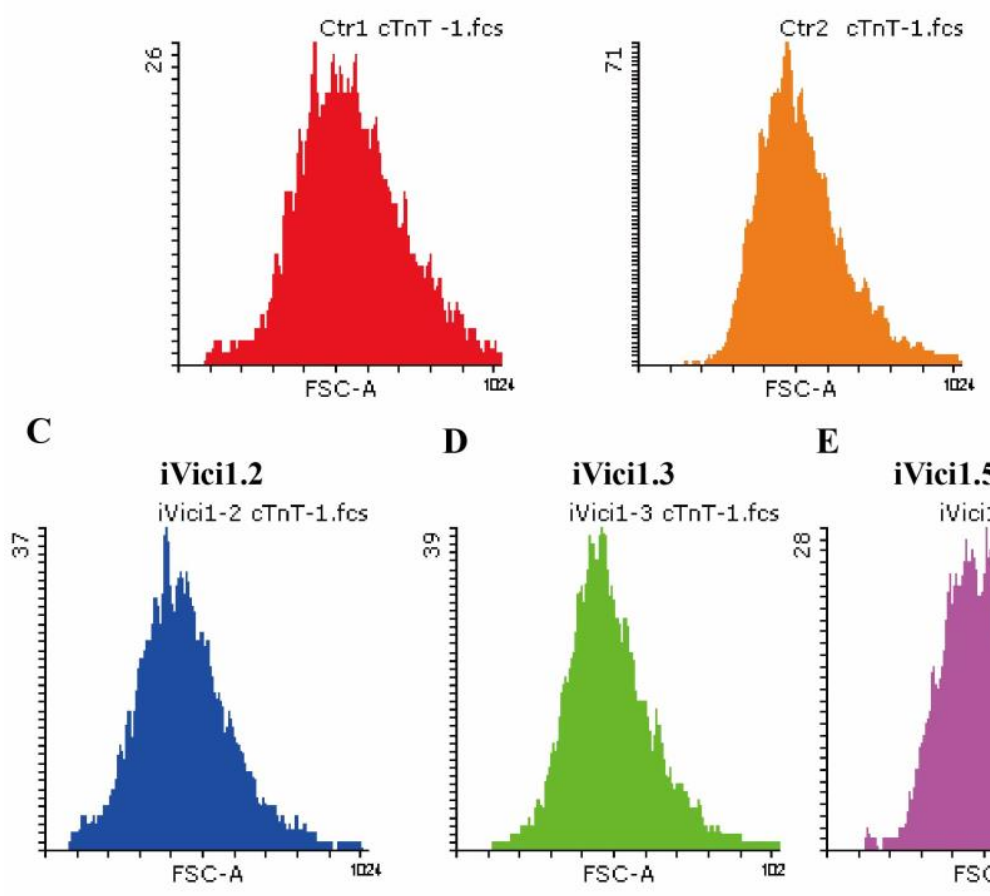

E

iVici1.3

iVici1.5
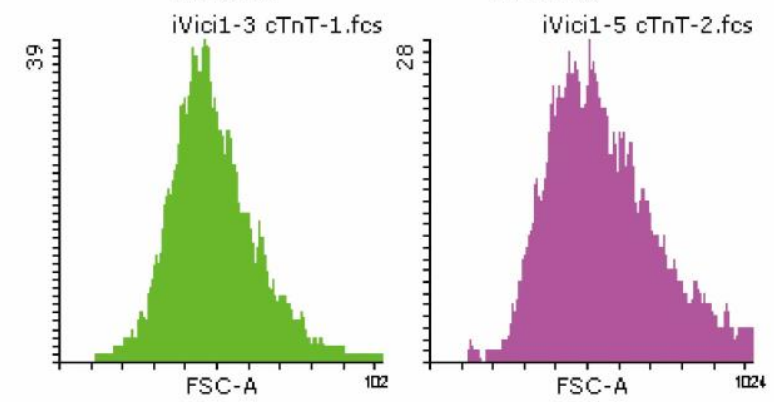

F

Overlap

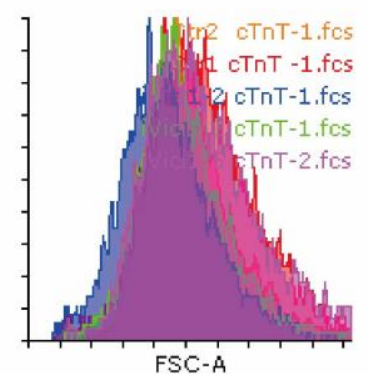

Figure 21. FSC distribution of Ctr- and Vici-iPSC-CMs. No significant differences of cell volumes were observed between Ctr- (A and B) and Vici-iPSC-CMs $(\mathbf{C}, \mathbf{D}$, and $\mathbf{E})$ as shown in the histogram overlap in FSC (F).

\subsubsection{Vici-iPSC-CMs exhibit multidirectional sarcomere organization but sarcomere lengths show no difference}

To evaluate the sarcomere length of hiPSC-CMs, the cardiomyocytes stained with $\alpha$-ACTININ were analyzed with Image J. Similar sarcomere length was observed between Ctr- $(1.765 \pm 0.017 \mu \mathrm{m}, \mathrm{n}=57)$ and Vici-iPSC-CMs $(1.830 \pm 0.023 \mu \mathrm{m}, \mathrm{n}=61)($ Figure 22). 


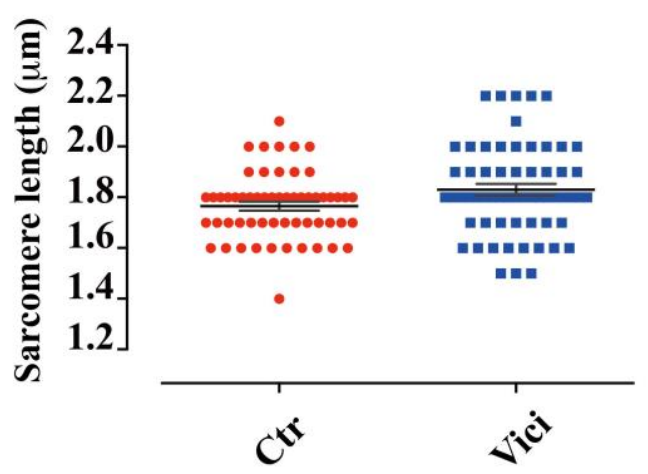

Figure 22. Similar sarcomere length in Ctr- and Vici-iPSC-CMs. The immunostaining images using the antibody against $\alpha$-ACTININ analyzed by Image $\mathrm{J}$ showed that there was no significant difference in the sarcomere length between Ctr- and Vici-iPSC-CMs.

Although the sarcomere length showed no significant difference between Ctr- and Vici-iPSC-CMs, Vici-iPSC-CMs were observed with complicated sarcomere structure under the microscope after the immunofluorescence staining against $\alpha$-ACTININ, a cardiac Z-disk component (Figure 23A and B). The sarcomere regularity analysis was carried out by the two-dimensional FFT. The amplitude of the first order peak showed no difference between Ctr- and Vici-iPSC-CMs, suggesting consistent regularity of sarcomeres in one direction (Figure 23D). The FWHM of the first-order peak was higher in Vici-iPSC-CMs compared with Ctr-iPSC-CMs, indicating the higher inhomogeneity of the sarcomere length in Vici-iPSC-CMs (Figure 23C). In addition, sarcomeres in Vici-iPSC-CMs distributed at different directions (Figure 23B). The $\alpha$-ACTININ structure was crossly distributed in a multidirectional way in the Vici-iPSC-CMs in contrast to Ctr-iPSC-CMs. 

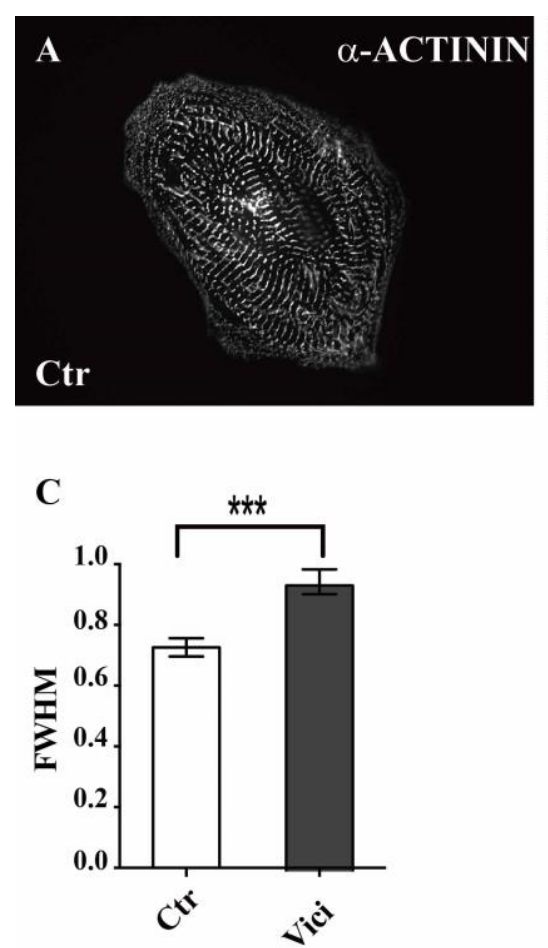

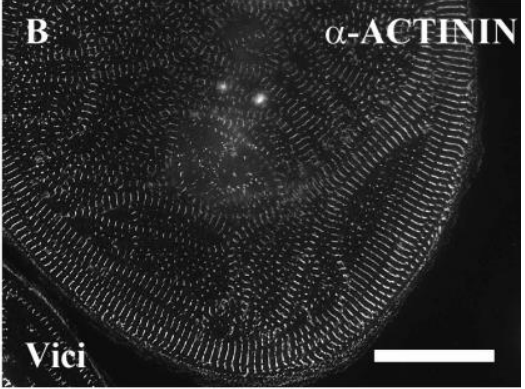

D

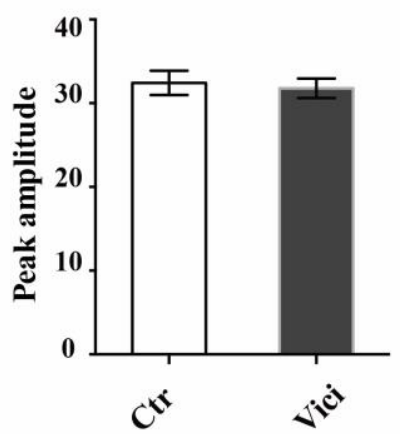

Figure 23. Quantitation of sarcomere organization of Ctr- and Vici-iPSC-CMs. The typical morphology of Ctr- and Vici-iPSC-CMs stained with $\alpha$-ACTININ (A and B). The sarcomere structure in Vici-iPSC-CMs had a multidirectional distribution. The quantitation of FWHM (C) and peak amplitude (D) analyzed with two-dimensional FFT showed higher inhomogeneity of the sarcomere length in Vici-iPSC-CMs. Scale bar: $50 \mu \mathrm{m}$.

Taken together, Vici-iPSC-CMs were observed to exhibit a large cell surface area but minimal difference in cell volumes compared to Ctr-iPSC-CMs. Vici-iPSC-CMs showed normal sarcomere length but with a multidirectional distribution based on the analysis of the sarcomeric protein $\alpha$-ACTININ.

\subsubsection{Functional analysis by generating engineered heart muscle}

On the basis of heightened multidirectional sarcomere structure in Vici-iPSC-CMs and to further investigate if autophagy defects can influence the functional contractility, EHMs were generated. Before used for the generation of EHMs, both Ctr- and Vici-iPSC-CMs were dissociated into single cells and the cell diameter was measured by using Roche Casy cell counter. The results showed no significant difference between Ctr-iPSC-CMs (20.51 \pm $0.72 \mu \mathrm{m})$ and Vici-iPSC-CMs $(19.79 \pm 0.77 \mu \mathrm{m})($ Figure 24). 


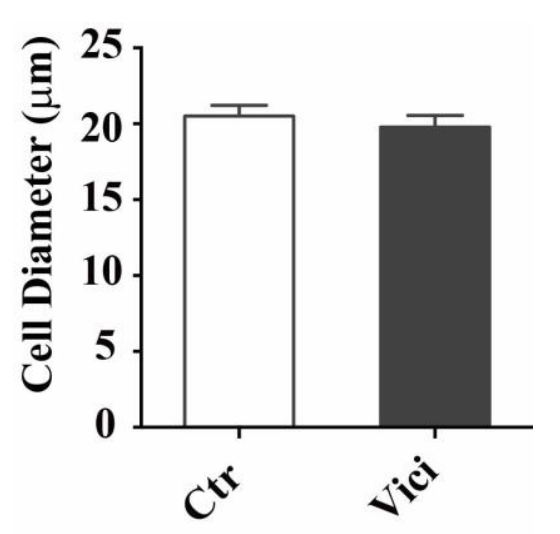

Figure 24. Cell diameter of Ctr- and Vici-iPSC-CMs before used for generiation of EHMs. The Vici-iPSC-CMs showed similar cell diameter to Ctr-iPSC-CMs.

EHMs generated from both Ctr- (Ctr-EHMs) and Vici-iPSC-CMs (Vici-EHMs) were stretched and further cultured for 2 weeks. Twitch tension of EHMs, expressed as force of contractions (FOC) in $\mathrm{mN}$, was measured in a thermostatted organ bath (Figure 25A). Additionally, forces developed in EHMs were measured over a dose response exposure to calcium concentrations from $0.2 \mathrm{mM}$ to $4 \mathrm{mM}$. The results revealed that the Vici-EHMs $(n=14)$ developed similar contractile forces compared with Ctr-EHMs $(n=15)$ (Figure 25B). Interestingly, the FOC relative to the cross-sectional area (CSA) was higher in Vici-EHMs than in Ctr-EHMs (Figure 25C and D). In order to elucidate whether higher FOC/CSA observed in Vici-EHMs was attributed to the diseased cardiomyocytes, Ctrl-EHMs and Vici-EHMs were digested into single cells and cellular composition (cardiomyocytes and non-myocytes) was analyzed by flow cytometry. Cells were stained with cardiac Z-disk binding protein $\alpha$-ACTININ on the day of measurement. The numbers of cardiomyocytes in EHMs were counted (Figure 25E) and the beating forces were calculated for a single cardiomyocyte. The data indicated that single cardiomyocytes in Vici-EHMs maintained the similar FOC to those in Ctr-EHMs (Figure 25F). In summary, there is no significant difference of functional contractility between Ctr- and Vici-EHMs. 


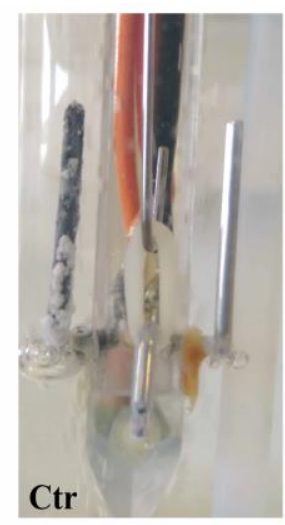

C

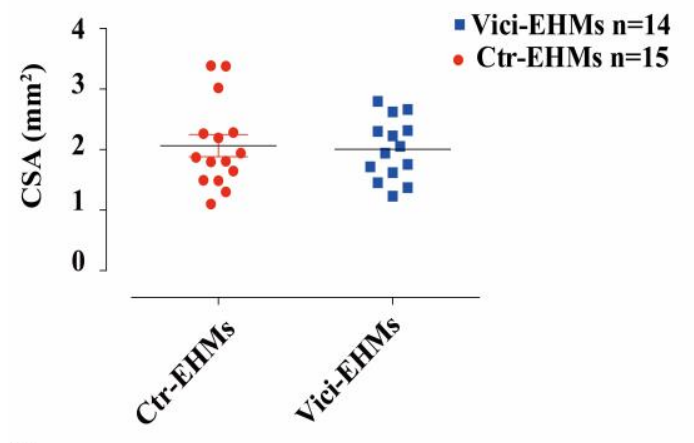

E

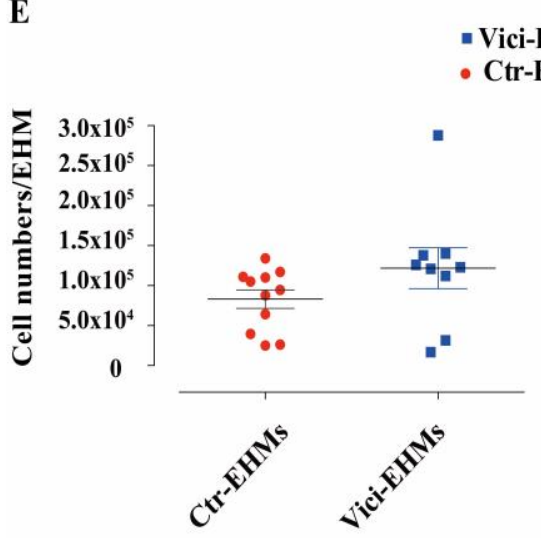

B

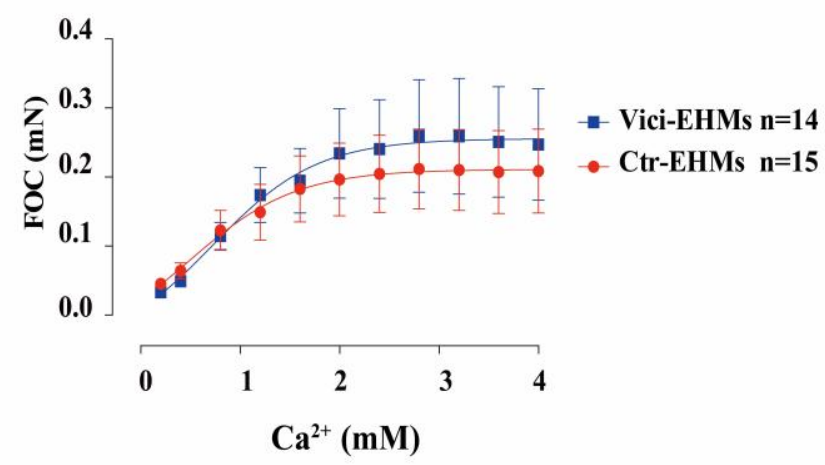

D

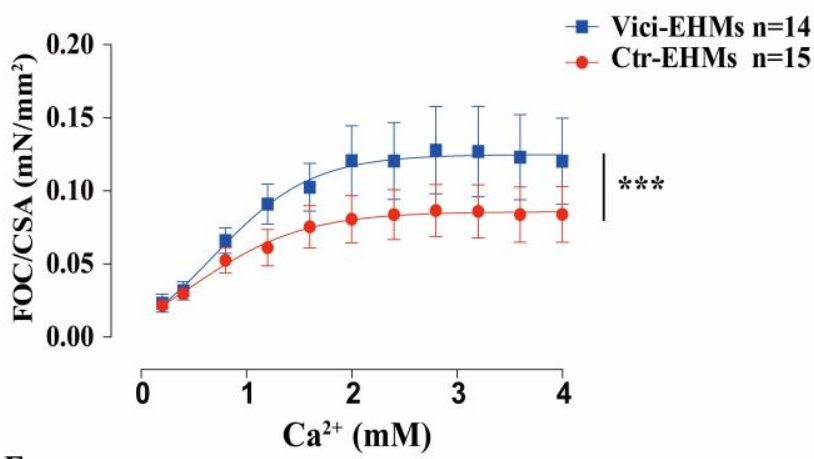

$\mathbf{F}$

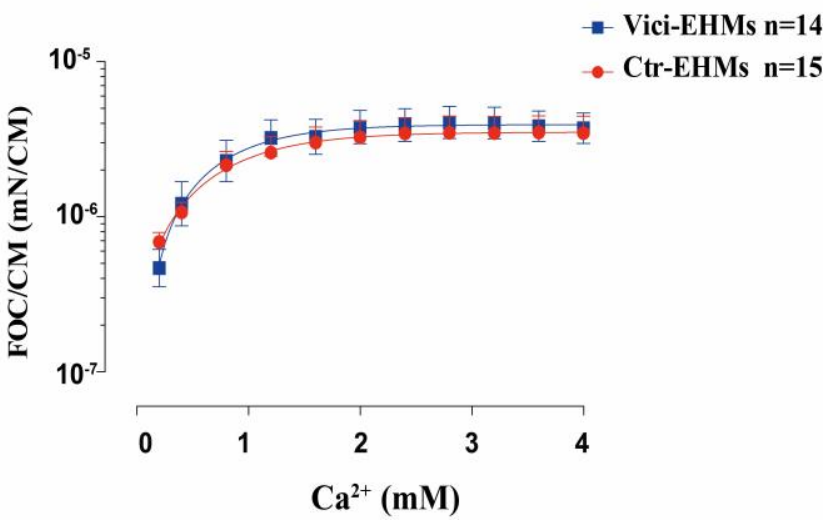

Figure 25. Measurement of contractile forces of EHMs generated from Ctr- and Vici-iPSC-CMs. A thermostatted organ bath was used for the measurement (A). Vici-EHMs $(n=14)$ showed similar absolute force of contractions (FOC) with extracellular calcium concentration compared with Ctr-EHMs $(\mathrm{n}=15)(\mathbf{B})$. The FOC per cross-sectional area (CSA) was higher in Vici-EHMs than in Ctr-EHMs ( $\mathbf{C}$ and $\mathbf{D})$ whereas the FOC per CM was similar in Vici-EHMs and Ctr-EHMs (E and $\mathbf{F})$. 


\subsubsection{Autophagy defect in Vici-iPSC-CMs}

\subsubsection{Accumulation of autophagy markers LC3 in Vici-iPSC-CMs}

To determine if cardiomyocytes show the autophagy defect, as observed in fibroblasts from the patient with Vici syndrome (Cullup et al., 2013), the expression levels of LC3, P62 and NBR1 were studied in Vici-iPSC-CMs compared to Ctr-iPSC-CMs by performing Western blot (Figure 26). The results indicated that untreated Vici-iPSC-CMs (Vici Nc) showed significantly increased expression levels of LC3 (LC3I and LC3II), in particular LC3II, the lipidated form of LC3, and slight increases of P62 and NBR1 in comparison to Ctr-iPSC-CMs (Ctr Nc).

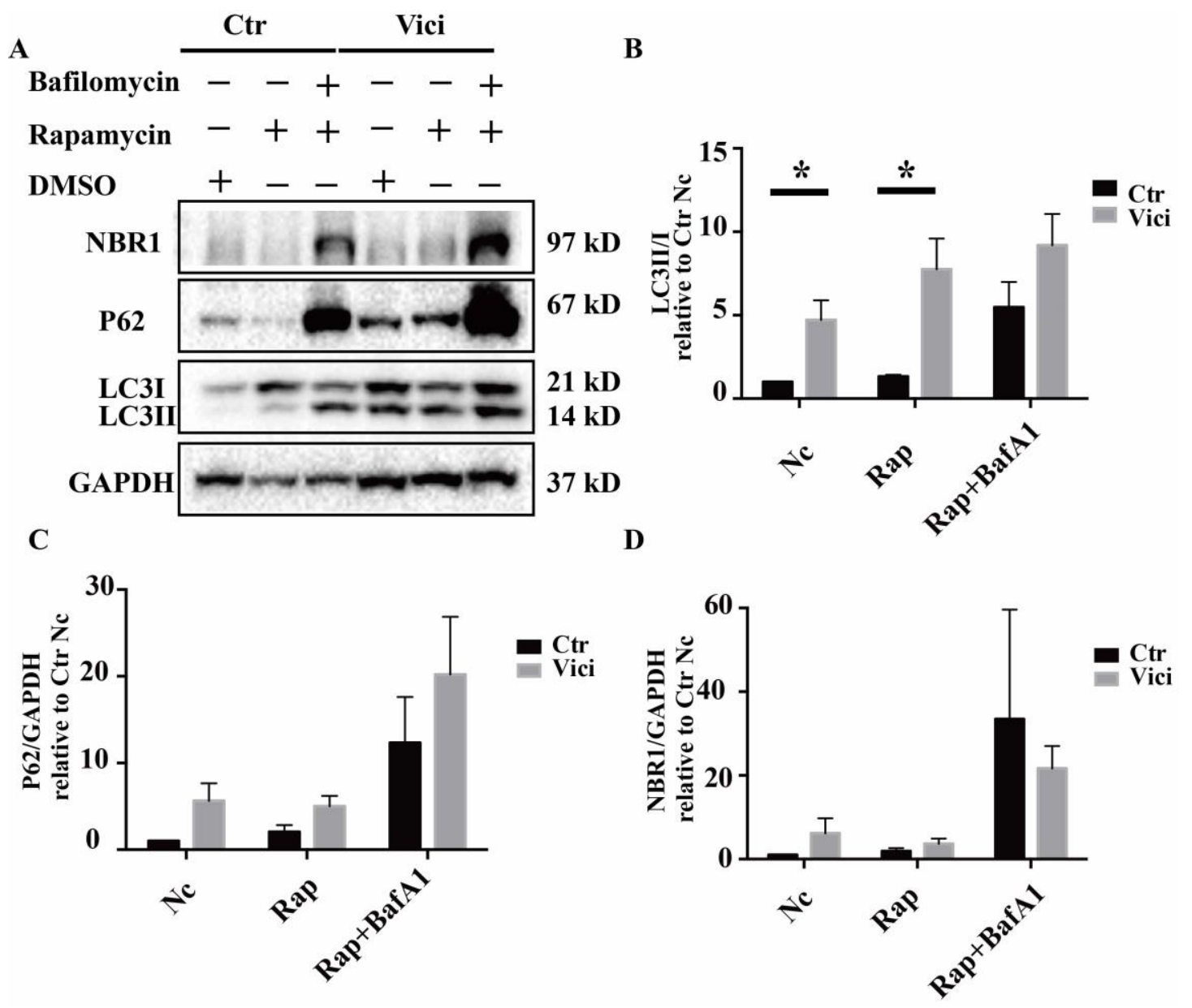

Figure 26. LC3I and LC3II were accumulated in Vici-iPSC-CMs. The autophagy adaptors P62 and NBR1 were only slightly accumulated whereas unprocessed LC3I and processed LC3II were significantly increased in untreated Vici-iPSC-CMs $(n=5)$ in comparison to Ctr-iPSC-CMs $(n=5)$. LC3 and the ratio of LC3II/I were increased after rapamycin and combined treatments in Ctr-iPSC-CMs. In contrast, no further accumulation of LC3I and LC3II was observed in Vici-iPSC-CMs after rapamycin and combined treatments (A and $\mathbf{B}$ ). Following the rapamycin treatment, P62 and NBR1 were slightly elevated in Ctr-iPSC-CMs, but not in Vici-iPSC-CMs, 
while the combined treatment led to a strong accumulation of these proteins in both Ctr- and Vici-iPSC-CMs (A, C and D).

In order to determine if LC3, P62 and NBR1 levels are reflective of increased upstream induction and/or downstream blockade of autophagy, Ctr- and Vici-iPSC-CMs were treated with the autophagy inducer rapamycin $(100 \mathrm{nM})$ and the autophagy blocker bafilomycin $(200 \mathrm{nM})$ for $12 \mathrm{~h}$. Rapamycin is one of the inhibitors of the mTORC1 complex. Bafilomycin is an inhibitor of the autolysosomal $\mathrm{H}^{+}$ATPase, which regulates the acidification and hence the degradation of proteins in lysosomes (Klionsky et al., 2012). In Ctr-iPSC-CMs, induction of autophagy by rapamycin led to slight accumulation of P62, NBR1 and LC3II/LC3I, while the combined upstream activation by rapamycin and the downstream blockade by bafilomycin resulted in further strong accumulation of these proteins (Figure 26A-D). In contrast, in Vici-iPSC-CMs, the expression levels of P62 and NBR1 were not further elevated following the rapamycin treatment, but enhanced after the combined treatment (Figure 26A, C, and D). Furthermore, the expression level of LC3 (LC3I and LC3II) was also not further increased after the rapamycin treatment, or the combined treatment. The ratio of LC3II/I in Vici-iPSC-CMs was slightly, but not significantly increased following the rapamycin treatment, and the combined treatment (Figure 26A, B). This suggests that there is no impairment in the induction of early processes in autophagy, including the processing of LC3I to LC3II, but that the clearance of autophagosomal cargo is nearly saturated in Vici-iPSC-CMs.

To further investigate the autophagy blockade in Vici syndrome, Ctr- and Vici-iPSC-CMs were exposed to the drugs of isoproterenol $(100 \mathrm{nM})$ and phenylephrine $(5 \mu \mathrm{M})$ for $12 \mathrm{~h}$ and in combination of them with rapamycin and bafilomycin. Isoproterenol is a non-selective $\beta$-adrenergic receptor agonist, and primarily used to treat bradycardia or heart block through increasing the heart rate (American Heart, 2006). Phenylephrine is a selective $\alpha_{1}$-adrenergic receptor agonist and decreases the heart rate through increasing blood pressure (Antonopoulos et al., 2002). The hiPSC-CMs showed the same response (increased or decreased beating rate, respectively) to both drugs (data not shown). In Ctr-iPSC-CMs treated with isoproterenol, phenylephrine, or their combination, the autophagy markers LC3I, LC3II and P62 were significantly higher expressed following the combined upstream activation by rapamycin and the downstream blockade by bafilomycin, only slightly induced after the induction of autophagy by rapamycin (Figure 27). 
However, in Vici-iPSC-CMs after the isoproterenol, phenylephrine, or the combined treatment, strong baseline accumulation of the unprocessed LC3I and processed LC3II was observed without rapamycin, or bafilomycin treatments. More interestingly, in Vici-iPSC-CMs treated with isoproterenol or phenylephrine, the expression level of LC3 (LC3I and LC3II), in particular LC3II, was further increased after the rapamycin treatment, or the combined treatment (Figure 27). Similar to Ctr-iPSC-CMs after the isoproterenol, phenylephrine, or the combined treatment, Vici-iPSC-CMs revealed marked accumulation of P62 after the dual induction of autophagy by rapamycin and block of autophagosomal degradation by bafilomycin (Figure 27).

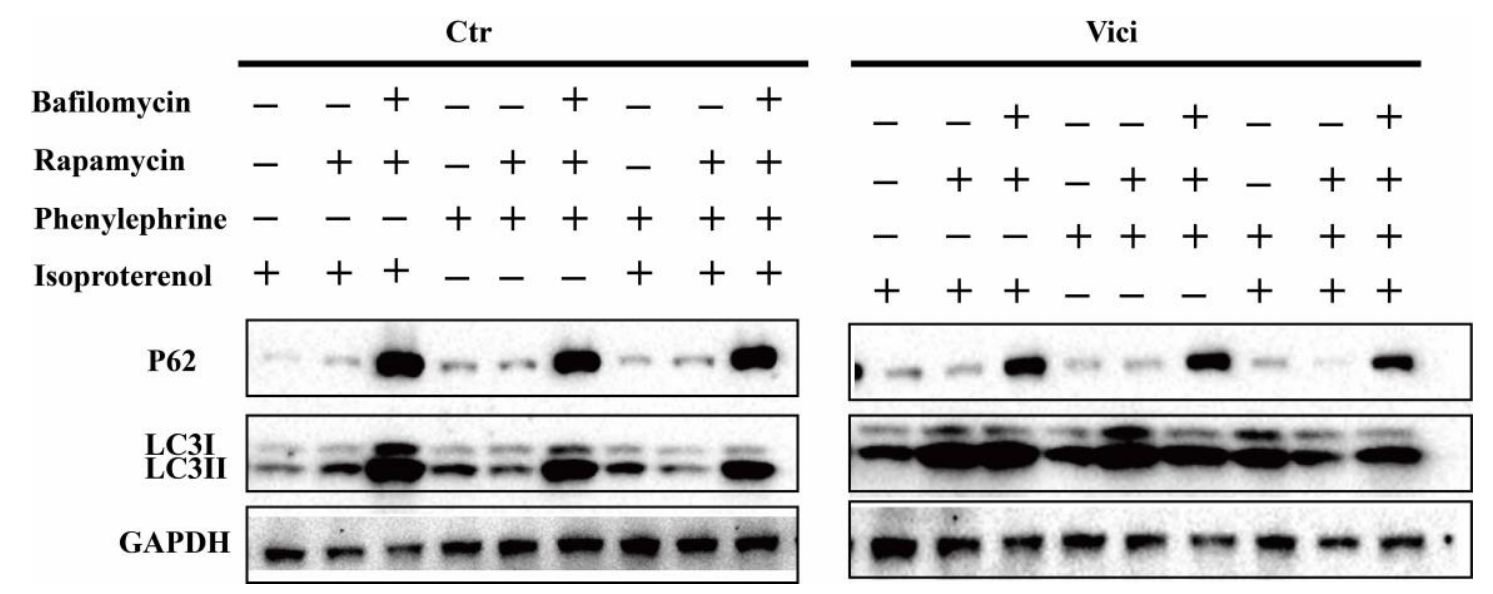

Figure 27. Enhanced expression of LC3 in Vici-iPSC-CMs after isoproterenol and phenylephrine stimulations. LC3I and LC3II were significantly accumulated in Vici-iPSC-CMs $(n=2)$ after isoproterenol and phenylephrine stimulations compared with Ctr-iPSC-CMs $(n=2)$ under basal, rapamycin and rapamycin and bafilomycin conditions.

Altogether, as a widely used marker of autophagy pathway, the enhanced LC3I and LC3II accumulation and conversion between LC3I and LC3II under normal and isoproterenol or phenylephrine stimulation conditions suggest that the autophagosomes might be aggregated in the Vici-iPSC-CMs.

\subsubsection{Autophagosome accumulation in Vici-iPSC-CMs}

The increased ratio of LC3II/I was detected by Western blot, suggesting the accumulation of autophagosomes in Vici-iPSC-CMs. In order to assess this hypothesis, immunofluorescence staining with antibodies against LC3 was performed. The cardiomyocytes were also stained with the antibody against the sarcomere protein $\alpha$-ACTININ. The analysis demonstrated that a low level of LC3II-positive puncta appeared in Ctr-iPSC-CMs. As expected, in the Vici-iPSC-CMs, there were numerous 
LC3II-positive puncta, mostly located around the nuclei (Figure 28).
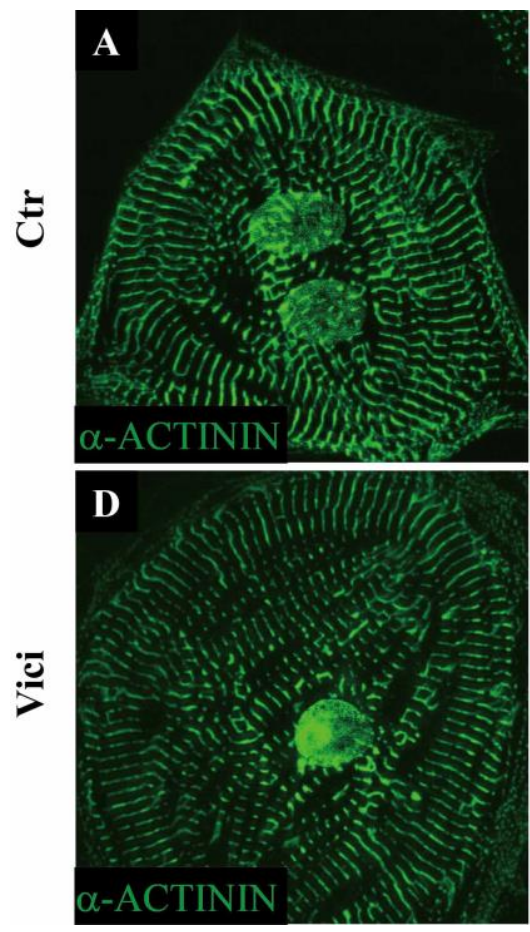

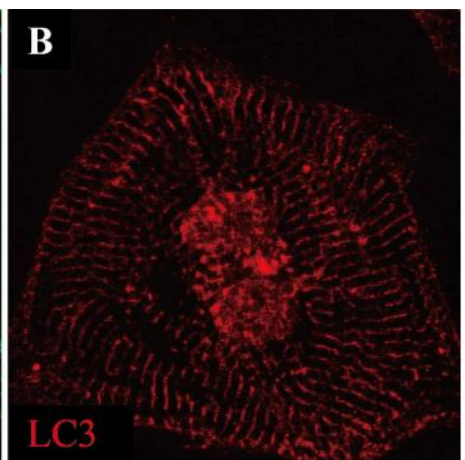

E

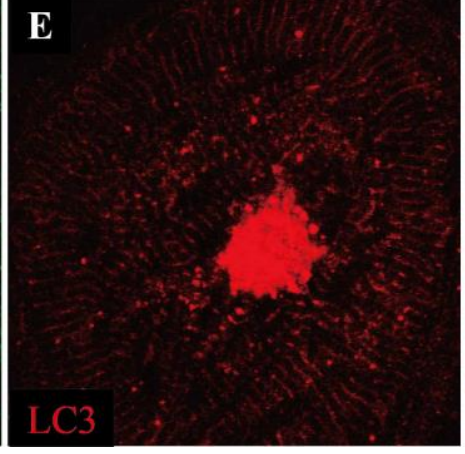

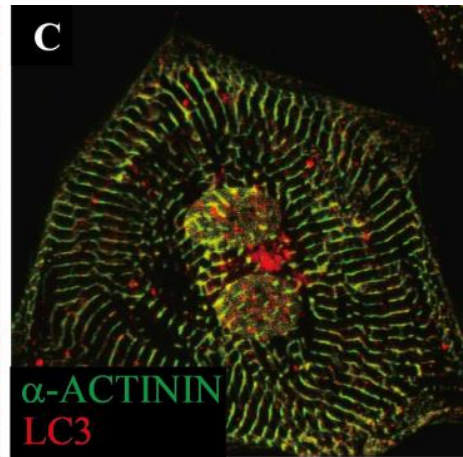

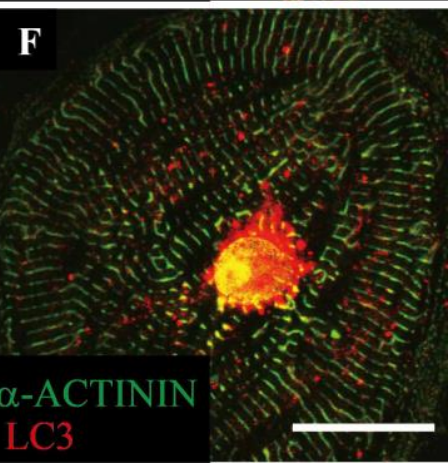

Figure 28. Accumulation of LC3II-positive puncta in Vici-iPSC-CMs in vitro. Immunofluorescence staining revealed that LC3II-positive puncta (red) were accumulated in Vici-iPSC-CMs (E) compared with Ctr-iPSC-CMs (B) under the normal condition (without the induction of autophagy or the blockade of clearance). $\alpha$-ACTININ (A, C, green) was stained as a cardiomyocyte marker. Scale bar: $50 \mu \mathrm{m}$.

To further confirm that autophagosomes are accumulated in Vici-iPSC-CMs and to rule out possible artifacts of immunostaining, the untreated cardiomyocytes were transfected with the LC3-GFP-RFP sensor. By using this sensor, the amount of autophagosomes is correlated with the number and intensity of the co-localized red and green fluorescent puncta, which represent the association of LC3II with autophagosomes (Zhang, 2015). The results showed that a high level of numbers puncta with the co-localization of red and green fluorescence existed in Vici-iPSC-CMs at baseline (Figure 29), suggesting the LC3-II localization to the autophagosome. These data indicates that autophagosomes are abnormally accumulated in the Vici-iPSC-CMs. 

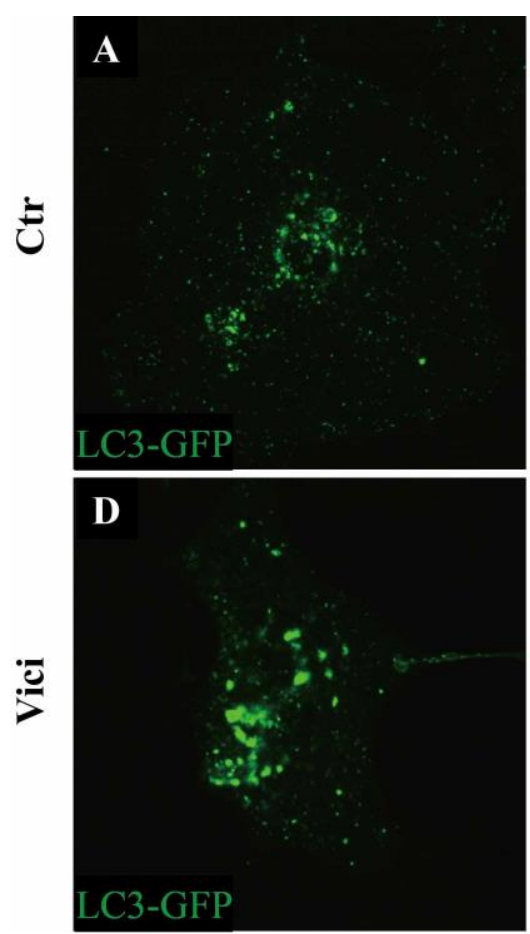
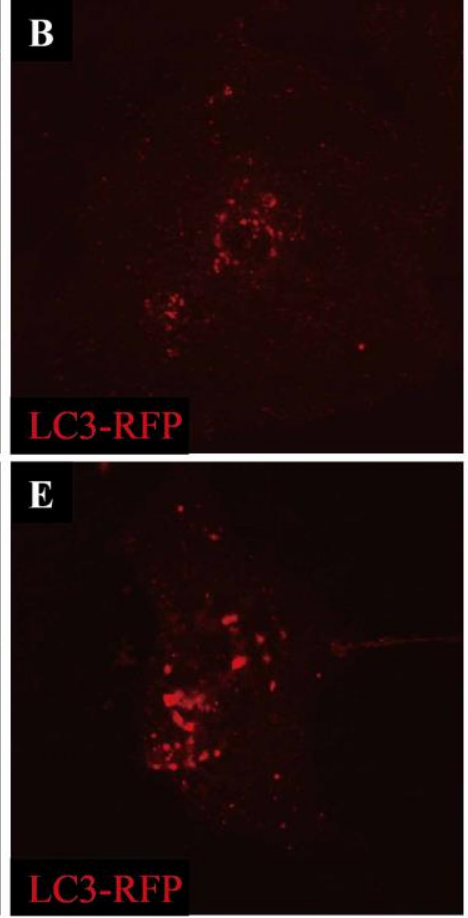
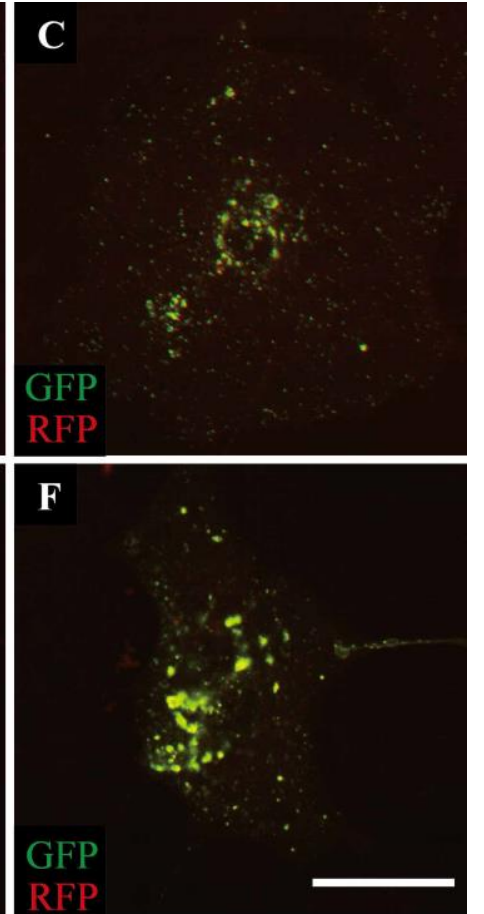

Figure 29. Accumulation of LC3-GFP-RFP in untreated Vici-iPSC-CMs. The untreated cardiomyocytes were transfected with the LC3-GFP-RFP sensor. The strong co-localization of red and green fluorescence signal in Vici-iPSC-CMs (D-F) revealed the accumulated autophagosomes compared with Ctr-iPSC-CMs (A-C). Scale bar: $50 \mu \mathrm{m}$.

\subsubsection{Abnormal localization of lysosomes in Vici-iPSC-CMs}

The lysosome is important for recycling and degrading proteins. Abnormalities of lysosomes can result in genetic diseases known as lysosome storage diseases. In previous study, the fibroblasts derived from the patient with Vici syndrome showed the fusion blockade of autophagosomes with lysosomes (Cullup et al., 2013). In order to determine the presence of lysosomes in the hiPSC-CMs, cardiomyocytes were stained with LAMP1. The results illustrated that the localization of LAMP1 between Ctr-iPSC-CMs and Vici-iPSC-CMs was totally different. In Ctr-iPSC-CMs, the LAMP1-positive puncta were mostly localized in the cytoplasm while only a small number were perinuclear (Figure 30A-C). In Vici-iPSC-CMs, the number of LAMP1-positive puncta was reduced while the size of the puncta was increased. Surprisingly, there were distinct perinuclear puncta (Figure 30D-F). The different localization of LAMP1-positive puncta indicates the abnormal distribution of lysosomes in Vici-iPSC-CMs. These data also imply that EPG5 may be involved in lysosome biogenesis and/or cellular trafficking in cardiomyocytes. 

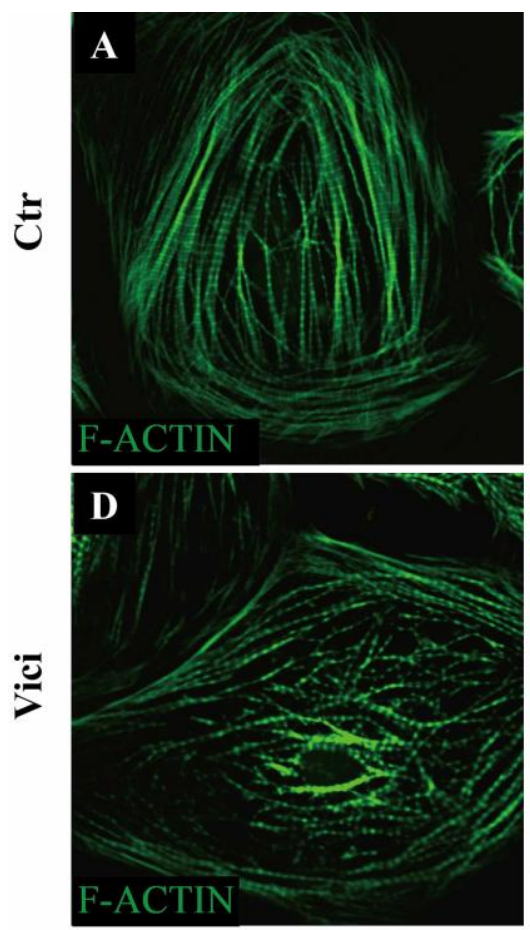
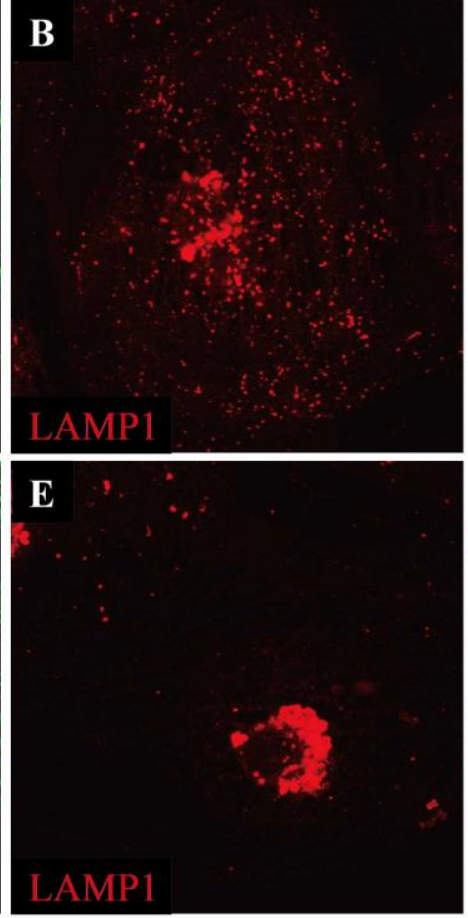
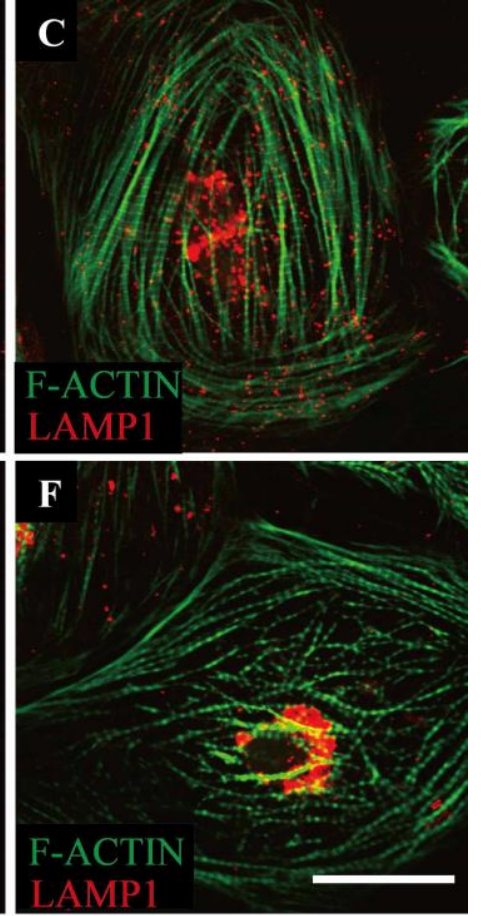

Figure 30. Abnormal localization of lysosomes in Vici-iPSC-CMs. Immunofluorescence staining was performed against LAMP1 (red). The results demonstrated that a few large lysosomes were located around the nucleus in Vici-iPSC-CMs (E) while many small lysosomes were distributed throughout the cytoplasm in Ctr-iPSC-CMs (B). Phalloidin (green) was used to label F-ACTIN in the cardiomyocytes. Scale bar: $50 \mu \mathrm{m}$.

\subsubsection{Fusion of autophagosomes with lysosomes was blocked in Vici-iPSC-CMs}

To study the fusion of autophagsomes with lysosomes, the cardiomyocytes were treated with autophagy inducer rapamycin and autophagy blocker bafilomycin for $12 \mathrm{~h}$ and were imaged after immunofluorescence staining of LC3 and LAMP1 (Figure 31). The LC3IIand LAMP1-positive puncta were significantly accumulated, and localized around the perinuclear region in both Ctr- and Vici-iPSC-CMs. In Ctr-iPSC-CMs, majority of the accumulated LC3II-positive puncta were found engulfed by the LAMP1-positive vesicular structures, suggesting that the autophagosomes were successfully fused with the lysosomes. In contrast, in Vici-iPSC-CMs, the co-localization of LAMP1-positive puncta with the LC3II-positive puncta was significantly reduced. Many LAMP1-positive vesicles without LC3II-positive puncta inside and many isolated LC3II-positive puncta were observed (Figure 31). These results indicate that there is impaired fusion of autophagosomes with lysosomes in EPG5 mutant cardiomyocytes. 

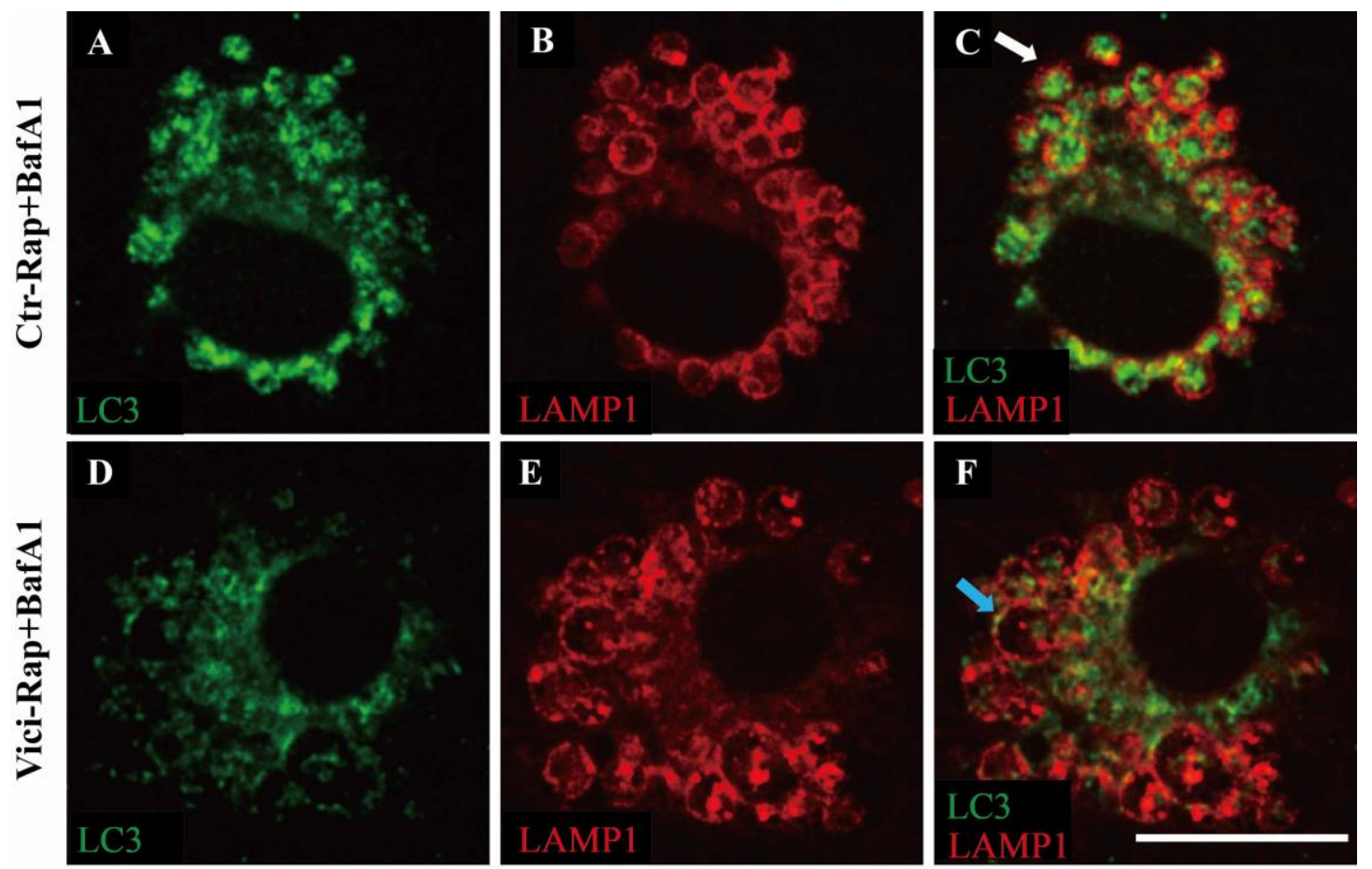

Figure 31. Blocked fusion of LC3II-positive puncta with LAMP1-positive puncta in Vici-iPSC-CMs. The LC3II-positive puncta (green) were engulfed by the LAMP1-positive vesicles (red) in Ctr-iPSC-CMs (A-C), whereas numerous LAMP1-positive vesicles were detected without LC3II-positive puncta in Vici-iPSC-CMs (D-F). The cardiomyocytes were subjected to rapamycin and bafilomycin for $12 \mathrm{~h}$. Scale bar: $50 \mu \mathrm{m}$.

\subsubsection{Colocalization of autophagy adaptor P62 and NBR1 in Vici-iPSC-CMs}

Expression levels of the autophagy adaptor P62 and NBR1 were slightly changed in Vici-iPSC-CMs, as detected by Western blot analysis. To see whether the distributions of the cargo-recognition proteins P62 and NBR1 are altered in Vici-iPSC-CMs, cardiomyocytes treated with rapamycin and bafilomycin were used for immunofluorescence staining. The results showed that both P62 and NBR1 were accumulated in Ctr- and Vici-iPSC-CMs after the rapamycin and bafilomycin treatment. The co-localization of P62-positive puncta and NBR1-positive puncta in Vici-iPSC-CMs (Figure 32 D-F) were found with the similar frequency to those observed in Ctr-iPSC-CMs (Figure 32 A-C). 

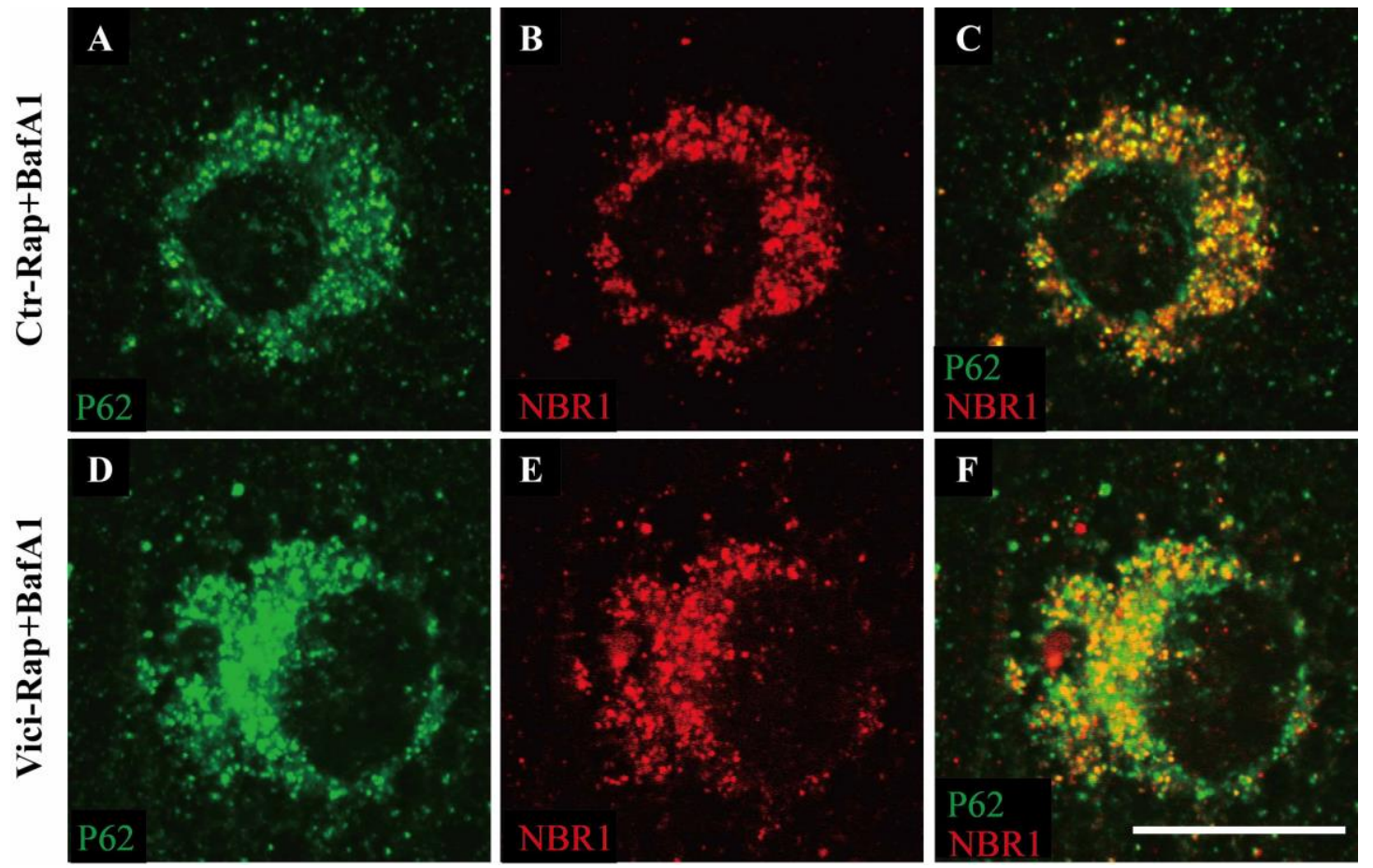

Figure 32. Colocalization of P62 and NBR1. The autophagy cargo-recognition proteins P62 (green) and NBR1 (red) were labeled in cardiomyocytes treated with rapamycin and bafilomycin. The accumulated P62 and NBR1 were visible in both Ctr-iPSC-CMs (A-C) and Vici-iPSC-CMs (D-F). P62 and NBR1 colocalized around the nucleus with the similar frequency in both cell types. Scale bar: $50 \mu \mathrm{m}$.

In addition, the staining of P62 and LAMP1 demonstrated that in Ctr-iPSC-CMs, P62-positive puncta were engulfted in LAMP1-positive puncta as the ring shaped structures. In Vici-iPSC-CMs, occasional fusion was observed in small P62-positive puncta and LAMP1-positive puncta. Many non-fused P62-positive puncta were observed (Figure 33). 

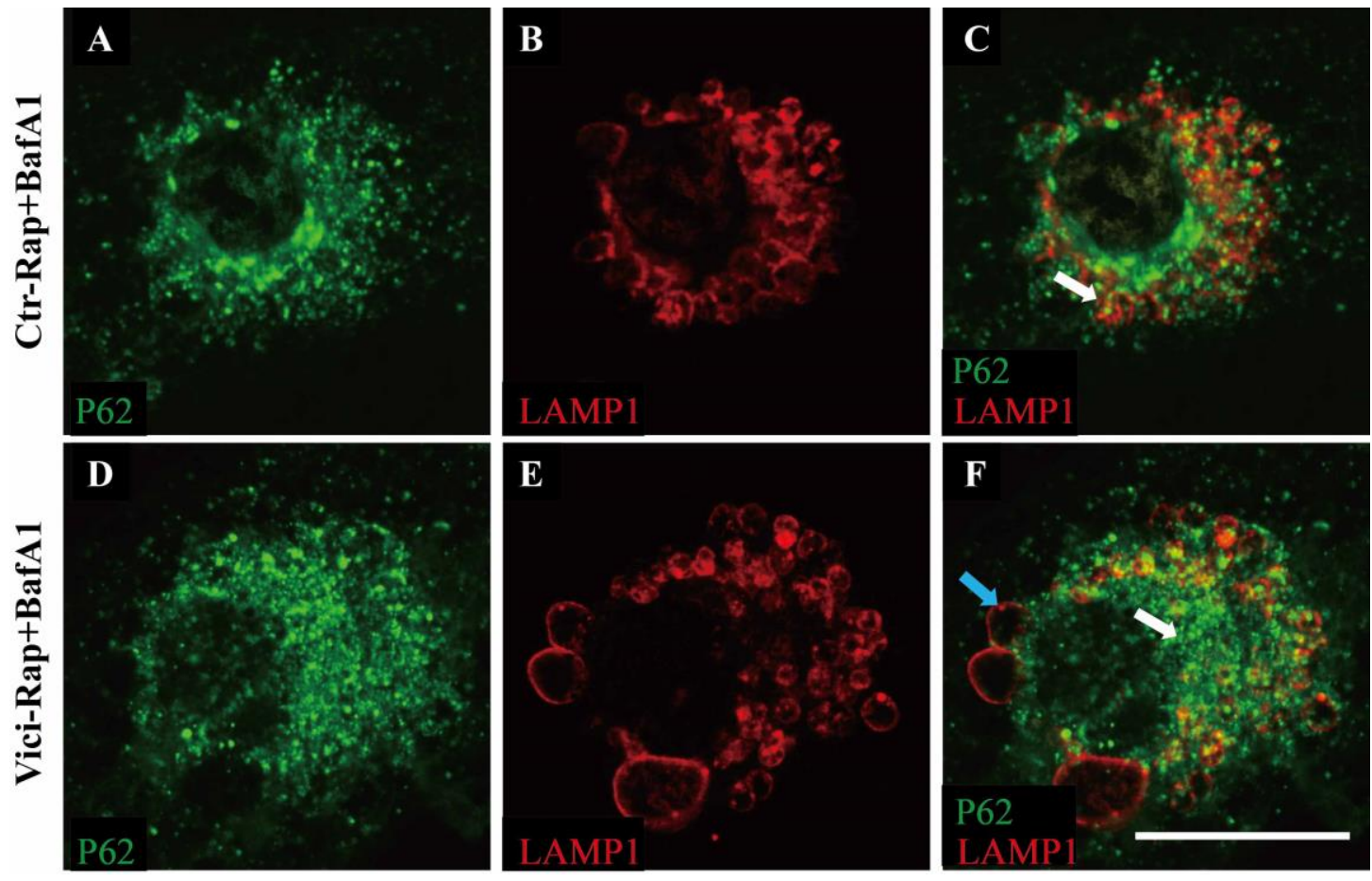

Figure 33. P62-positive puncta fused with lysosomes. Immunofluorescence staining was performed after rapamycin and bafilomycin treatment. In control cells (A-C), P62 (green) fused with LAMP1 (red) as a ring-shaped structure. In patient cells (D-F), P62 fused with LAMP1 as small puncta and some non-fused P62 were observed. Scale bar: $50 \mu \mathrm{m}$.

Taken together, the expression and localization of P62 and NBR1 in the Ctr-iPSC-CMs and Vici-iPSC-CMs were similar indicating that the formation of autophagy cargo is normal in Vici-iPSC-CMs. The abnormal fusion of P62- and LAMP1-positive structures in Vici-iPSC-CMs suggests that the function of EPG5 is mostly involved in the fusion of autophagosomes with lysosomes.

\subsubsection{Mitochondrial localization in hiPSC-CMs}

Electron microscopy analysis of skeletal muscle biopsy from this patient showed variable size, abnormal distribution and morphology of mitochondria (Cullup et al., 2013). Mitochondria are important for cell survival by generating adenosine triphosphate (ATP). To compare the mitochondrial localization in Vici-iPSC-CMs with Ctr-iPSC-CMs, the cells were stained with the antibody against COXIV, which catalyzes the last step in electron transfer chain of the mitochondria. Preliminary data showed similar distribution of mitochondria in Ctr-iPSC-CMs and Vici-iPSC-CMs, as indicated by the similar localization of COXIV. In addition, COXIV-positive structures were not accumulated in LAMP1-labeled lysosomes in Vici-iPSC-CMs similar to Ctr-iPSC-CMs (Figure 34). 

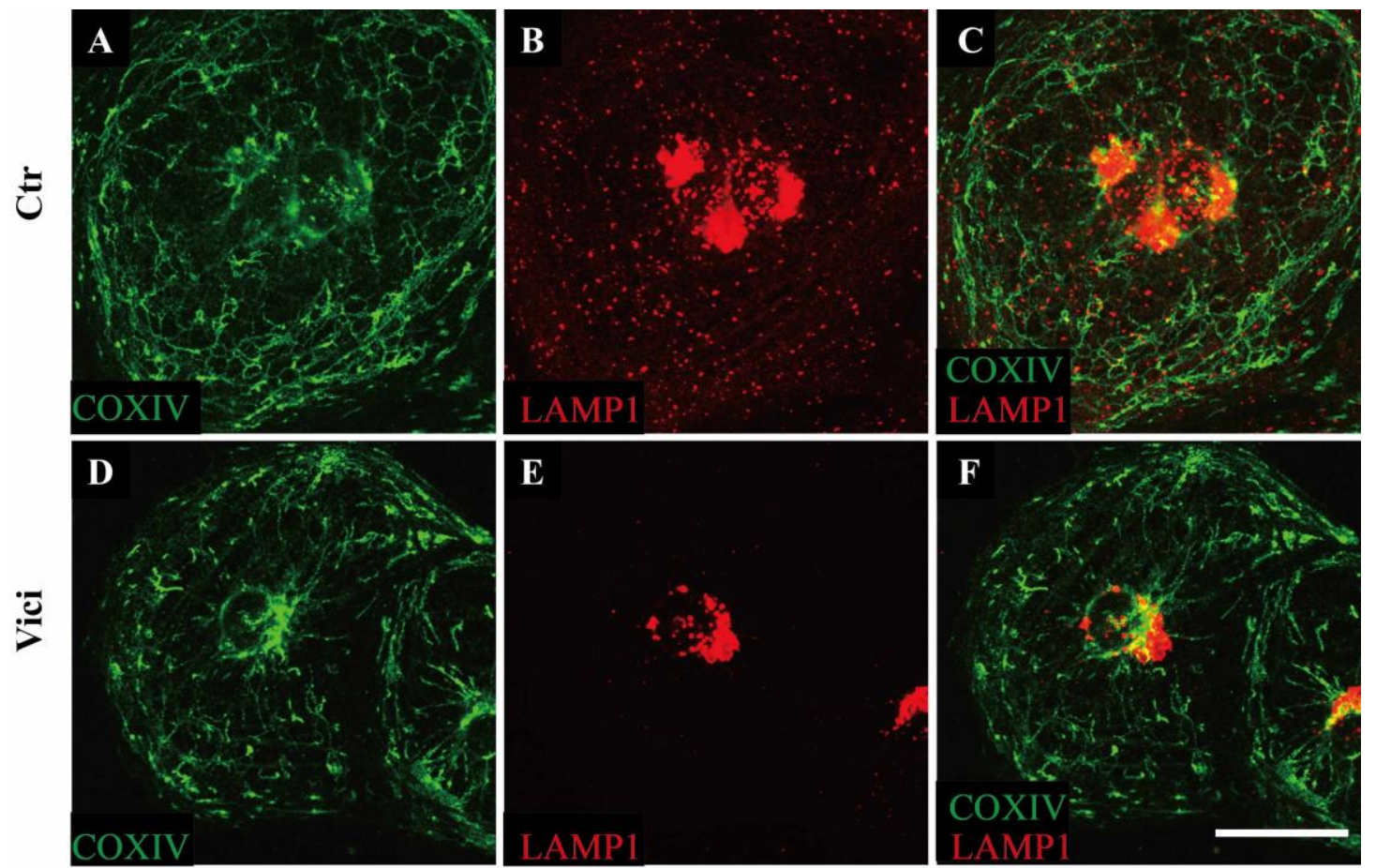

Figure 34. Mitochondrial distribution in Ctr- and Vici-iPSC-CMs. The cardiomyocytes were stained with COXIV (green) and LAMP1 (red). There was no obvious accumulation of mitochondria in Vici-iPSC-CMs (D-F) compared with Ctr-iPSC-CMs (A-C). Scale bar: $50 \mu \mathrm{m}$.

In summary, these data illustrate a serious defect of autophagy in Vici-iPSC-CMs. Enhanced expression of LC3I and LC3II as well as numerous LC3II positive puncta were detected in the untreated Vici-iPSC-CMs demonstrated by Western blots and immunofluorescence staining. After the dual treatment with rapamycin and bafilomycin, empty lysosomes without any autophagy cargo were visible in the Vici-iPSC-CMs, shown by double stainging of LAMP1 and LC3 or LAMP1 and P62 in the cells. Furthermore, the abnormal localization of LAMP1-positive structures in Vici-iPSC-CMs suggests that EPG5 may be involved in the lysosome biogenesis and/or cellular trafficking. However, there is no obvious difference of the expression and localization of the autophagy adaptors P62 and NBR1. In addition, the distribution of mitochondria in Vici-iPSC-CMs is comparable to that in Ctr-iPSC-CMs.

\subsubsection{Abnormal AKT pathway in Vici-iPSC-CMs}

To determine if the abnormality observed in Vici-iPSC-CMs involves changes in the regulatory signal pathway of autophagy, the classical PI3K/AKT/mTOR/ pathway was elucidated in this study. This pathway regulates many cellular processes such as cellular 
metabolism, cell proliferation, cell growth, survival, apoptosis, gene transcription, protein synthesis and so on. It controls the expression of autophagy proteins by inhibiting the transcriptional activity of FoxO transcription factors (Daitoku et al., 2011).

First, the investigation of phosphorylation of AKT showed significantly reduced phosphorylation of two activating sites of AKT (AKT S473 and AKT T308) in both untreated and treated (rapamycin, rapamycin with bafilomycin) Vici-iPSC-CMs in comparison to those in Ctr-iPSC-CMs (Figure 35).

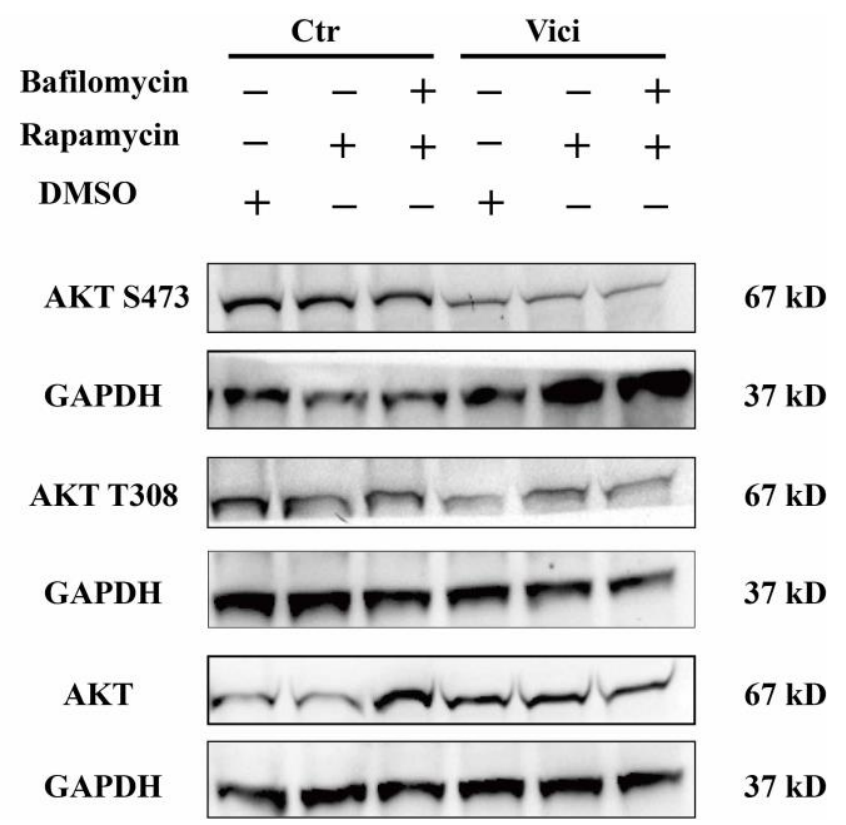

Figure 35. Reduced phosphorylation of AKT S473 and AKT T308 in Vici-iPSC-CMs. Phosphorylation of two activating sites of AKT serine 473 (S473) and threonine 308 (T308) were reduced in Vici-iPSC-CMs under basal conditions, rapamycin treatment, and combined treatment with rapamycin and bafilomycin.

Second, phosphorylation of the downstream proteins of AKT including FOXO3 and FOXO1 were assessed. The phosphorylation of activating sites of FOXO3 (FOXO3 T32) and FOXO1 (FOXO1 T24) were also dramatically decreased in both treated and untreated (rapamycin, rapamycin with bafilomycin) Vici-iPSC-CMs compared to Ctr- iPSC-CMs (Figure 36). 


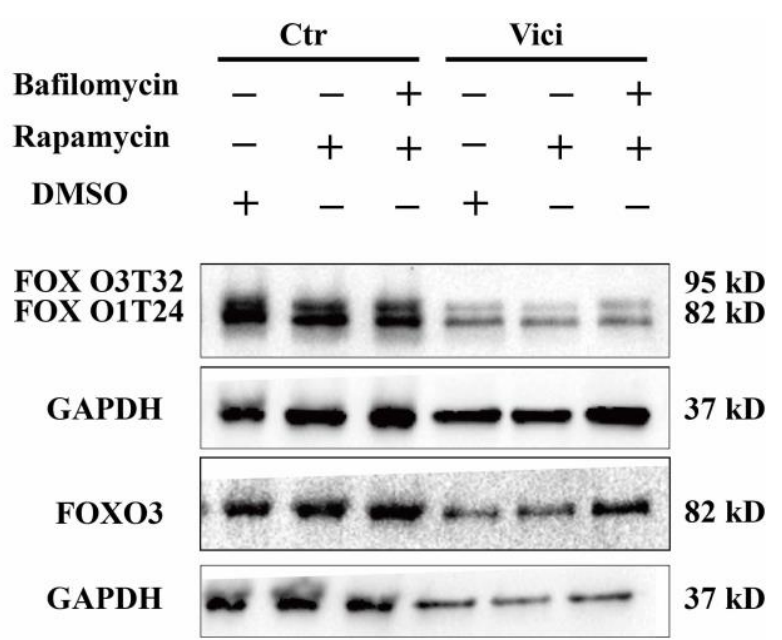

Figure 36. Decreased phosphorylation of FOXO3 threonine 32 (T32) and FOXO1 threonine 24 (T24) in Vici-iPSC-CMs.

In addition, the analysis of total and phosphorylation of mTOR protein was performed. The data showed that treatments with rapamycin or rapamycin and bafilomycin resulted in decrease of total mTOR and mTOR S2448 phosphorylation in Ctr-iPSC-CMs, as expected (Figure 37). In Vici-iPSC-CMs, marked baseline reduction of total mTOR and mTOR S2448 phosphorylation compared to Ctr-iPSC-CMs was observed. Treatments with rapamycin or rapamycin and bafilomycin led to further slight reduction of mTOR S2448 phosphorylation (Figure 37).

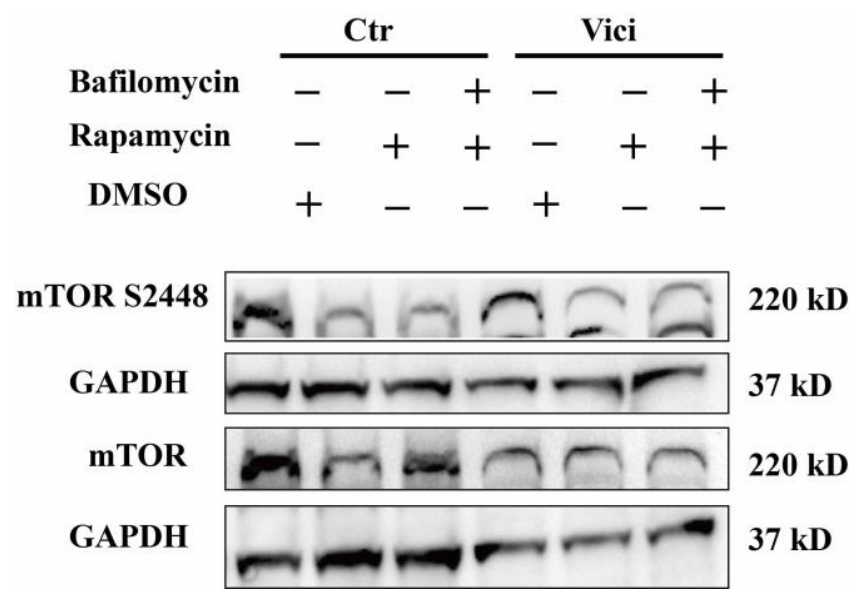

Figure 37. mTOR expression and phosphorylation in Vici-iPSC-CMs. In both control and patient cells, the expression of total mTOR and phosphorylation of mTOR (S2448) were reduced after rapamycin, or rapamycin and bafilomycin dual treatment. In addition, the total mTOR and mTOR S2448 were reduced under basal condition in Vici-iPSC-CMs compared with Ctr-iPSC-CMs.

Moreover, P70S6K, which is one of the direct downstream regulatory proteins of mTOR, 
was analyzed. The results demonstrated that the expression and phosphorylation (T389) of P70S6K was similar in untreated Vici-iPSC-CMs and Ctr-iPSC-CMs (Figure 38). The low phosphorylation of P70S6K was not observed after rapamycin treatment or dual treatment with rapamycin and bafilomycin in both Ctr- and Vici-iPSC-CMs.

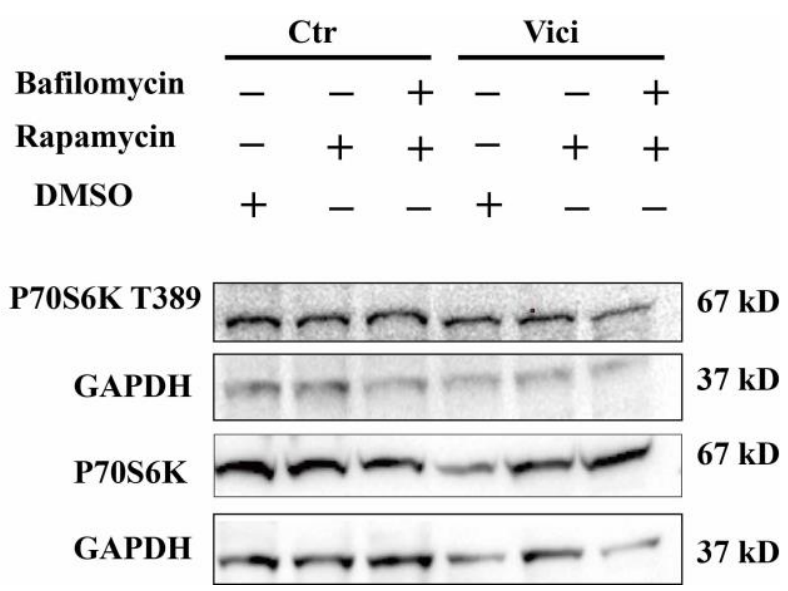

Figure 38. Phosphorylation of P70S6K in Ctr-iPSC-CMs and Vici-iPSC-CMs. The assessment of phosphorylation of P70S6K threonine 389 (T389), a direct downstream target of mTOR, showed similar expression in both Ctr-iPSC-CMs and Vici-iPSC-CMs.

Furthermore, the preliminary analysis of GSK3 $\beta$ showed that total expression of GSK3 $\beta$ and phosphorylation of GSK3 $321 / 9$ seemed to be similar in both Ctr- and Vici-iPSC-CMs (Figure 39).

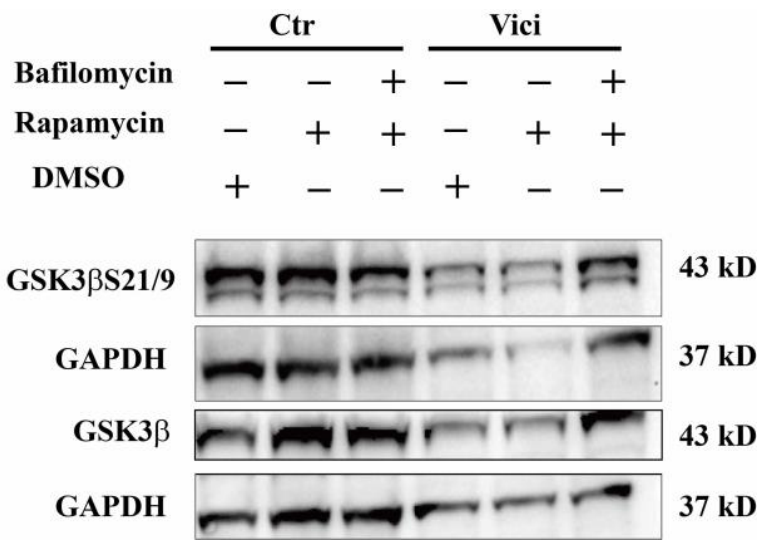

Figure 39. Phosphorylation of GSK3 $\beta$ in Ctr- and Vici-iPSC-CMs. The expression of total GSK $3 \beta$ and phosphorylation of GSK3 $\beta$ at two activating sites serine 21 and 9 (GSK3 $\beta$ S21/9) seemed to be no significant difference between Ctr- and Vici-iPSC-CMs.

In summary, the abnormalities of the AKT/mTOR classic pathway including reduced phosphorylation of AKT T308, AKT S473, FOXO3 T32, FOXO1 T24, and mTOR S2448 were found in Vici-iPSC-CMs compared to Ctr-iPSC-CMs. Base on the preliminary data, 
no big differences of the expression and phosphorylation of P70S6K and GSK3 $\beta$ were found in Ctr- and Vici-iPSC-CMs. The irregular regulation, in particular phosphorylation of AKT may not only cause the autophagy defect in Vici-iPSC-CMs but also lead to other multiple dysfunctions in EPG5 mutant cardiomyocytes. However, it is still unknown if the EPG5 mutation was the only causative factor for the abnormal phenotypes observed in Vici-iPSC-CMs.

\subsection{Gene editing by CRISPR/Cas9 technology}

To determine that the abnormalities of sarcomere structure and autophagy defects in Vici-iPSC-CMs was only due to the EPG5 mutation, oligonucleotide-based gene editing via the CRISPR/Cas9 technology was applied to "rectify" the mutation in the EPG5 gene in Vici-iPSCs. Initially, targeting sgRNA nearby the mutation site in EPG5 $(c .4952+1 \mathrm{G}>\mathrm{A})$ were designed. The computational prediction of potential off-target sites of Cas9 were performed (by Sigma). Only one EPG5 sgRNA (Figure 40A) at 34 bp before the EPG5 mutation site was found free of off-target with up to $\mathrm{n}=2$ mismatches. This sgRNA was then cloned into the CRISPR/Cas9 system.

The 150 bp-long single-stranded oligonucleotides of EPG5 as donor templates (Figure 40A) were designed and used to replace the mutation site through homologous recombination. In the donor oligonucleotides, 6 synonymous substitutions were made in the region of protospacer adjacent motif (PAM) and $\operatorname{sgRNA}$ binding sites to prevent donor DNA from cleavage by Cas9 in the edited hiPSCs, and to aid the distinction between the donor and wild-type sequences, respectively. The synonymous substitutions resulted in the introduction of a new restriction enzyme site Hind III in the sgRNA. Afterwards, the mutated nucleotide was also changed from adenine (A) to guanine $(\mathrm{G})$. Then, a stepwise protocol was established to edit EPG5 in Vici-iPSCs (Figure 40B). Briefly, first, the CRISPR/Cas9-GFP plasmid was transfected into HEK293T cells and the cleavage assay was used to determine the efficiency of the CRISPR/Cas9 system. Second, the CRISPR/Cas9 cassette was transfected using nucleofection into Vici-iPSCs and single living GFP-positive cells were sorted. Thereafter, sorted $\mathrm{GFP}^{+}$single Vici-iPSCs were cultured until sufficient pure colonies available and genomic DNA from these colonies was 
extracted for sequencing.

A

gRNA

PAM

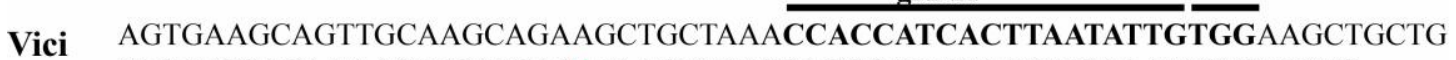
TACATGCAGAAAACTTGATCACATAAGTTTTGCAGGCATTATAATTTGAAATACTGTTTTG

HindIII

Oligo AGTGAAGCAGTTGCAAGCAGAAGCTGCTAAACCACCAAGCTTAAATATTGTAGAAGCTGCTG TACATGCAGAAAACTTGATCACGTAAGTTTTGCAGGCATTATAATTTGAAATACTGTTTTG

B

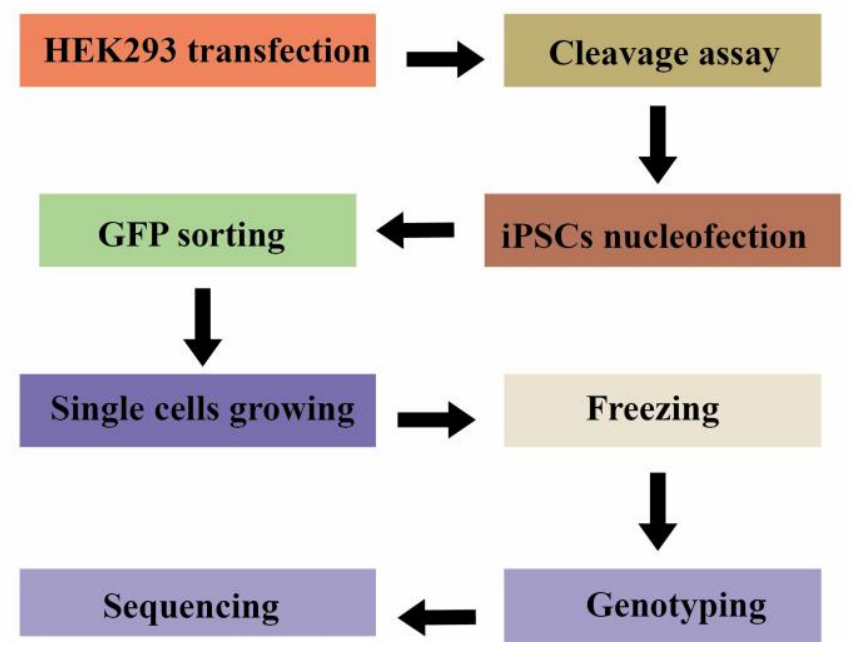

Figure 40. The scheme of correcting the mutation in EPG5 by using the CRISPR/Cas9 technology. The PAM motif and sgRNA was chosen $31 \mathrm{bp}$ and $34 \mathrm{bp}$ before the point mutation (green), respectively. The oligonucleotides with the corrected point mutation and synonymous mutations were synthesized (red) (A). The flowchart of the technique was shown in $\mathbf{B}$.

After the CRISPR/Cas9-GFP vector was transfected into HEK293T cells, GFP ${ }^{+}$cells were observed $48 \mathrm{~h}$ later (Figure 41A, B). The efficiency of successful CRISPR/Cas9 editing, recognizing and cutting the target DNA was analyzed using enzymatic cleavage assay (Figure 41C). The results showed that the efficiency was approximately $31 \%$ compared to $21 \%$ in the positive control (Figure 41D). 


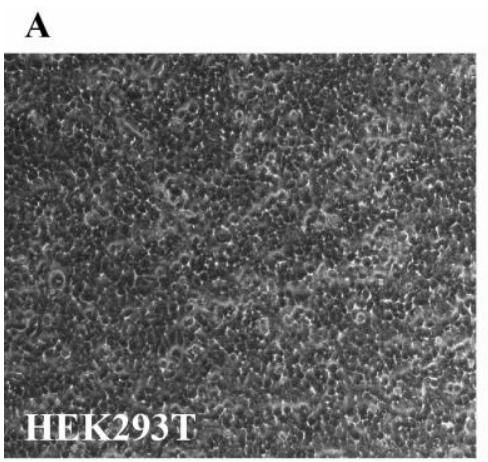

B

C

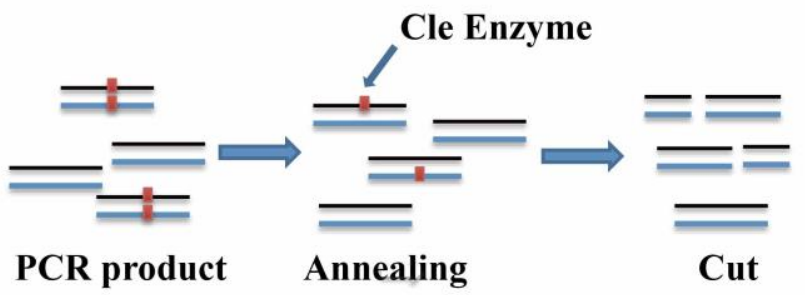

D

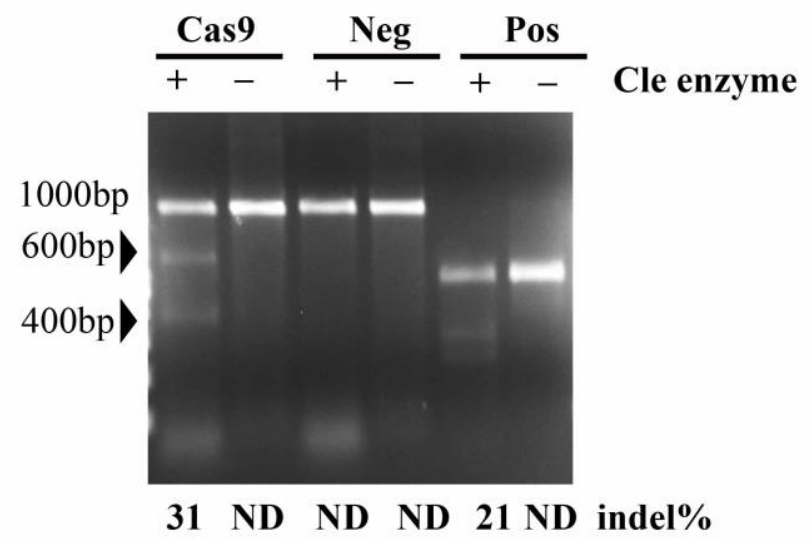

Figure 41. Assessment of cleavage efficiency of CRISPR/Cas9-GFP. The CRISPR/Cas9-GFP was transfected into HEK293T cells (A, B). The efficiency of CRISPR/Cas9-GFP confirmed by cleavage assay was around 31\% (C, D). Non-transfected HEK293T cells were used as negative control while the positive control was used from the commercial kit.

Next, the CRISPR/Cas9-GFP plasmid was transfected into Vici-iPSCs. However, GFP fluorescence was weak and the efficiency of nucleofection in iPSCs was relative low, about 10-15\% (Figure 42A). Sorted $\mathrm{GFP}^{+}$single cells were subsequently cultured and amplified. Altogether, $150 \mathrm{GFP}^{+}$colonies were obtained, from which genomic DNA was extracted. The PCR results showed the abnormal pattern of the EPG5 gene in 4 colonies (Figure 42B), suggesting that NHEJ happened in the cells to repair the DNA double-stranded break caused by the CRISPR/Cas9 cutting. Furthermore, after restriction digestion of the PCR products by Hind III, it was inferred that EPG5 was targeted in two colonies. However, the DNA sequencing results revealed that the inserted Hind III in both clones was generated by NHEJ rather than homologous recombination (Figure 42C). NHEJ resulted in nucleotide deletion forming a new Hind III site. 


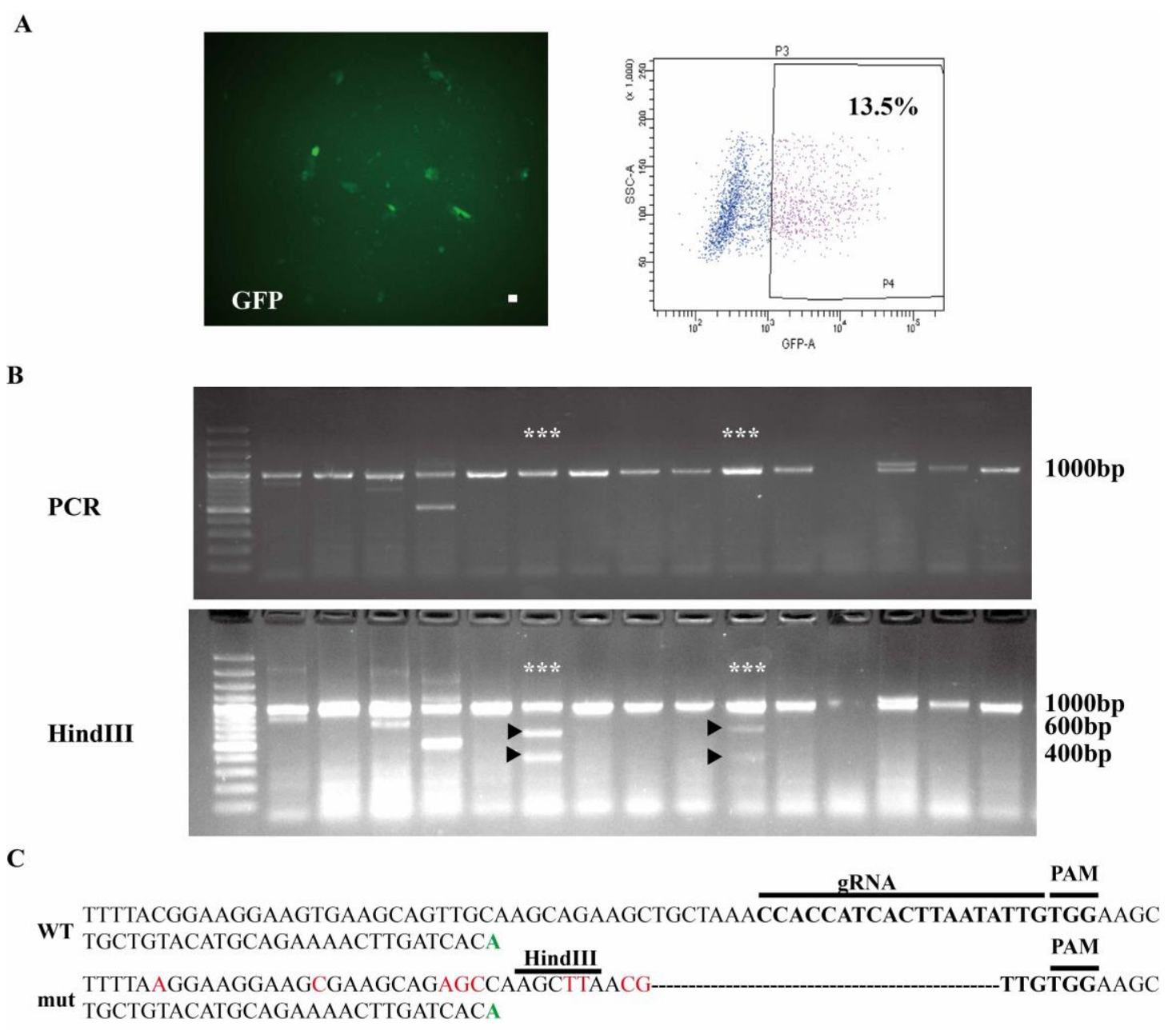

Figure 42. Representative clones after the genomic editing. The efficiency of Vici-iPSCs nucleofected with CRISPR/Cas9-GFP was around 13.5\% (A). Different genomic DNA PCR products were observed in different clones $(\mathbf{B})$. The sequence of the PCR product that could be cut by Hind III contained 20bp nucleotide deletion in the genomic DNA $(\mathbf{C})$.

Taken together, the data showed that the CRISPR/Cas9-GFP system functioned in Vici-iPSCs to introduce DNA double-stranded break with a low efficiency. Among 150 $\mathrm{GFP}^{+}$colonies generated, in none of them the mutated EPG5 was "corrected" with homologous recombination. 


\section{Discussion}

In this study, the patient-specific iPSC platform was used to investigate the Vici syndrome associated cardiomyopathy and the underlying molecular mechanism in vitro. First, Vici-iPSCs were generated from a patient with Vici syndrome with a homozygous intronic mutation in EPG5 gene $($ c. $4952+1 \mathrm{G}>\mathrm{A})$. Ctr-iPSCs used in this study were generated from healthy controls as previously published (Dudek et al., 2013; Liu et al., 2016; Streckfuss-Bomeke et al., 2013). Positive alkaline phosphatase staining, high expression of pluripotent-related markers and spontaneous differentiation into derivatives of three germ layers in vitro and in vivo demonstrated the pluripotency of the three generated and analyzed Vici-iPSC lines. Furthermore, the Ctr- and Vici-iPSCs were differentiated into functional cardiomyocytes, shown as spontaneous contraction with expression of cardiac-related markers. Structural analysis of cardiomyocytes revealed that Vici-iPSC-CMs displayed the normal sarcomere length but exhibited a multidirectional sarcomere organization. Although the cell volume of Vici-iPSC-CMs was comparable with that of Ctr-iPSC-CMs, the cell surface area of Vici-iPSC-CMs was significantly larger compared with Ctr-iPSC-CMs. Analysis of force of contraction in EHM showed a similar concentration-dependent positive inotropic response to increasing extracellular calcium concentrations in Vici-EHMs and Ctr-EHMs. In Vici-iPSC-CMs, strong baseline accumulation of LC3I and LC3II as well as increased ratio of conversion between LC3II and LC3I was visible that was largely unresponsive to the treatment with rapamycin and bafilomycin. Autophagosome accumulation (as indicated by LC3II-positive puncta) and abnormal localization of lysosomes (as indicated by LAMP1-positive puncta) in untreated Vici-iPSC-CMs as well as the fusion blockade of autophagosomes with lysosomes after the rapamycin and bafilomycin treatment further illustrated the autophagic disorder in Vici-iPSC-CMs. Investigation of the main autophagy regulation pathway (PI3K/AKT/mTOR) revealed that phosphorylation of AKT (T308 and S473), FOXO3 (T32)/FOXO1 (T24) and mTOR (S2428) was downregulated in Vici-iPSC-CMs, indicating that the PI3K/AKT/mTOR pathway is involved in the autophagy defect in Vici-iPSC-CMs. Furthermore, in this study, the CRISPR/Cas9 technique was used to 
rectify the mutation in EPG5 for rescuing the phenotypes observed in Vici-iPSC-CMs. Although no isogenic Vici-iPSCs with the corrected EPG5 gene were generated, the data indicate that the CRISPR/Cas9 technique functions in Vici-iPSCs.

\subsection{The generated Vici-iPSCs are pluripotent}

In this project, iPSCs were generated from the patient with Vici syndrome with the EPG5 mutation (c.4952+1G>A) and three iPSC lines were proved to be pluripotent. However, the iPSC generation from the patient with this rare disease was complex. Fibroblasts derived from the patient with Vici syndrome proliferated slowly in vitro and showed a planar morphology and significantly larger size compared to normal healthy fibroblasts (data not shown). Different methods including Sendai virus and STEMCCA lentivirus transduction as well as plasmid transfection to deliver the transcription factors OCT4, SOX2, KLF4 and c-MYC were applied to generate Vici-iPSCs, however, Vici-iPSCs could be generated only through the STEMCCA lentivirus transduction. The difficulty of transducing of Vici fibroblasts perhaps is due to the slow proliferation of fibroblasts, which may result from the autophagy defect (Cullup et al. 2013). Additionally, autophagy is required for reprogramming of somatic cells into iPSCs. The delicate balance of mTORC1 inhibition and autophagy induction coordinated by OCT4, SOX2, KLF4 and c-MYC in the early phase of reprogramming determines the reprogramming efficiency (Wang et al., 2015; Wang et al., 2013; Wu et al., 2015).

Sendai virus and plasmid nucleofection are advantageous to generate safe iPSCs for further understanding disease phenotype and the underlying molecular mechanism because of carrying no risk of altering host genome (Fusaki et al., 2009; Si-Tayeb et al., 2010). However, in this study, Vici syndrome-specific fibroblasts deteriorated 4-6 days after Sendai virus transduction. Previous studies showed that apoptotic cell death could be induced by blocking the autophagic flux in different cell types and autophagy plays functional roles in defence in infections (see review by (Marino et al., 2014)). In addition, virus infect could inhibit the autophagic flux (Granato et al. 2014, Metz et al. 2015). The death observed in Vici fibroblasts after Sendai virus infection might be due to the cell 
death induced by the block of the autophagic flux in Vici fibroblasts resulted from the the EPG5 mutation (Cullup et al. 2013), which might be further enhanced by the Sendai virus infection. Compared to the Sendai virus system, the STEMCCA lentivirus transduction is advantageous, (1) because it contains the four necessary transcription factors in a single polycistronic vector so that less virus particles are needed for the successful reprogramming; and (2) because the STEMCCA lentivirus can infect both diving and non-dividing cells, especially, when taking the slow proliferation rate of Vici fibroblasts into account. But STEMCCA lentivirus possesses the risks to disrupt the genomic information and leads to insertional mutagenesis because of the random integration into host genome (Somers et al., 2010).

Proof of pluripotency showed that all analyzed iPSC lines expressed high levels of pluripotent-related markers such as OCT4, SOX2, KLF4, LIN28, NANOG and SSEA4. In vitro differentiation of Vici-iPSCs showed expression of specific markers for different cell types derived from all three embryonic germ layers, illustrating that they are pluripotent. Teratomas derived from Vici-iPSCs were small and histological analysis showed that these teratomas contained intestinal tissue (endoderm), muscle-like cells (mesoderm) and neural-like cells (ectoderm). The formation of teratoma is a gold standard to prove the pluripotency of iPSCs (Przyborski, 2005). However, the reason for the small teratoma formation is unclear; perhaps it results from cell apoptosis after injection or autophagy defect (Nelakanti et al., 2015; Zhang et al., 2008).

In this study, the remodeling of epigenetic markers during reprogramming of somatic cells into pluripotent state, such as the demethylation on the promoters of key endogenous pluripotent markers OCT4 and NANOG (Marson et al., 2008), is not determined in the Vici-iPSCs and needs to be further investigated.

Genomic stability during long-term culture of iPSCs is important for analyzing the relationship between the disease phenotype and gene mutation. Previous study showed that genomic instability occurs in all cell types in long-term culture and karyotypic 
aberrations in hiPSC cultures are similar to those of hESCs (Martins-Taylor and $\mathrm{Xu}$, 2012). Recurrent aneuploidies were detected during the prolonged culture of hESCs with G-banded karyotyping, including trisomy 12, trisomy $\mathrm{X}$ (female hESC lines) and amplification of chromosome 17 (Draper et al., 2004). To ensure genetic stability in the cell lines used in this study, they were used at passages earlier than 35 . The majority (95\%) of Vici-iPSCs analyzed with G-banded karyotyping at 30 passages exhibited a normal diploid karyotype with 46 chromosomes (data not shown). But the traditional G-banded analysis has its limitations; its capability is limited to larger chromosomal aberration detection and failure to detect amplification and deletion of small regions. New technologies including fluorescent in situ hybridization, array-based comparative genomic hybridization and single-nucleotide polymorphism based microarray can be applied to detect karyotypic abnormalities and copy number variations.

The mutation $(c .5921+1 \mathrm{G}>\mathrm{A})$ in the EPG5 gene was verified by sequencing in the analyzed Vici-iPSCs and the missing of exon 28 in EPG5 was confirmed by RT-PCR on mRNA level. However, in order to figure out if EPG5 deficit is the only reason to cause the disease phenotype, the genomic DNA should be sequenced because the STEMCCA lentivirus-based reprogramming used in this study was possible to generate unknown mutations in the host genome.

Taken together, these generated Vici-iPSCs have similar characteristics as hESCs regarding to the morphology, expression of pluripotent-related genes and capability of differentiation. With the same genetic background of the patient with Vici syndrome, Vici-iPSCs carrying the EPG5 mutation are suitable for modeling Vici syndrome and uncovering the molecular mechanism of the disease.

\subsection{Cardiomyocytes derived from hiPSCs are embryonic-like cells}

In this study, cardiomyocytes at late stages (60-90 days old) were used for studying the disease phenotype of Vici syndrome in vitro. HiPSC-CMs provide significant advances for studying the cardiac disease and treatment due to their unlimited production from 
patient-specific iPSCs. Cardiomyogenic differentiation protocols via modulating of the Wnt signaling pathway by CHIR99021 and IWP2 yield cardiomyocytes with a high purity (over 90\%). Majority (more than $80 \%$ ) of the cardiomyocytes are ventricular-like cells. However, hiPSC-CMs possess molecular signature and phenotypes that resemble embryonic cardiomyocytes rather than adult cardiomyocytes (Robertson et al., 2013). First, most of hiPSC-CMs appeared as a small and rounded morphology with mono-nuclei while adult CMs are large and cylindrical (rod-like morphology) with double or multiple nuclei. In addition, the extensive T-tubule network found in mature adult cardiomyocytes is absent in hiPSC-CMs. These networks are responsible for the excitation-contraction coupling involving precise communication between L-type calcium channel located mainly on the T-tubules and calcium release channels/ryanodine receptor channels on the sarcoplasmic reticulum in adult CMs. However, calcium in hiPSC-CMs primarily enters the cell through sarcolemma and results in a slow excitation-contraction coupling (Lieu et al., 2009). For energy supply, the hiPSC-CMs depend on glycolysis for the production of ATP and could metabolize lactate and glucose whereas adult CMs mainly utilize oxidative metabolism (Tohyama et al., 2013).

To induce their maturation similar to adult cardiomyocytes, hiPSC-CMs were cultured for extended time (80-120 days post initial beating), resulting in maturation of their structure, contractile elements and electrophysiology to a more adult-like phenotype compared with early stage (20-40 days post initial beating) cell cultures (Lundy et al., 2013). The authors demonstrated that hiPSC-CMs at late stages showed high expression of cardiac structural genes, greater myofibril density and alignment, increased calcium release and reuptake rates without maximum amplitude changes, and better electrophysiology properties (hyperpolarized maximum diastolic potentials, increased action potential amplitude and faster upstroke velocities). However, although the long-term culture could enhance the maturation of hiPSC-CMs, the pool of differentiated cells still contains a heterogeneous population of cells that recapitulate some features of embryonic cardiomyocytes. Modifying the cardiomyocyte culture condition would enhance their maturation in vitro. For instance, Tri-iodo-L-thyronine (T3), a growth hormone that is expressed in neonatal 
cardiomyocytes and enhances normal cardiac maturation in vivo, could enhance the maturation of hiPSC-CMs (Yang et al., 2014). It remains unclear what is the best approach to enhance the maturity of hiPSC-CMs. Despite current immature states of hiPSC-CMs, these cells still possess great potential for modeling phenotypes of cardiac diseases, especially for the diseases like Vici syndrome, which is an early-onset disease.

\subsection{Vici-iPSC-CMs recapitulate the cardiac phenotype of the patient with Vici syndrome}

\subsubsection{Vici-iPSC-CMs show disorganized sarcomere structures}

More than $80 \%$ of patients with Vici syndrome possess a hypertrophic or dilated cardiomyopathy especially in the left ventricle ((Byrne et al. 2016a). In this study, when attached to the cell culture dish, Vici-iPSC-CMs displayed larger cell surface area than Ctr-iPSC-CMs, but without showing an increased cell volume when detached. Similar phenomenon was observed together with an increased viscoelasticity in cardiac fibroblasts after the downregulation of RhoA (Jatho A et al. 2015). However, it is largely unclear why Vici-iPSC-CMs show this phenomenon, and did not show an increase in cell volume as expected. One explanation can be that the cells are still too young to demonstrate the hypertrophic phenotype. Previous study showed that the ratio of surface area/volume of cardiomyocytes undergoes significant developmental changes (Satoh $\mathrm{H}$ et al. 1996). Furthermore, many complicated and diverse signaling pathways regulate cell sizes in different cell types. Dividing and non-dividing adult cell maintains a constant cell size in a homeostatic state for balancing the synthesis and degradation of macromolecules (Koivusalo et al., 2009). The PI3K/AKT/mTORC1 pathway is mainly involved in the regulation of autophagy and studies have demonstrated that this pathway regulates cell proliferation and is also a key determinant of cell size, with the artificial activation of this pathway during development resulting in larger animals that could result from hypertrophic cells (Edgar, 2006; Laplante and Sabatini, 2012; Tumaneng et al., 2012). Over-activation of mTOR may cause accumulation of defective cellular structures and organelles, which may result in age-related cardiac hypertrophy (Lloyd, 2013; Loffredo et 
al., 2013).

Hypertrophic cardiomyopathy is normally characterized by increased cell size, heightened organization of sarcomere and enhanced protein synthesis at the cellular level (Frey et al., 2004). Sarcomeric analysis showed that Vici-iPSC-CMs exhibited normal sarcomere length but higher inhomogeneity of sarcomere length. This, however, did not result in a significant functional consequence (no significant difference in force of contraction) from the analysis of EHMs. Evidence of such disorganization of sarcomere arrangements was demonstrated in mice with the tamoxifen-inducible cardiac-specific knockout of autophagy related atg5, in which cardiomyocytes showed disorganized sarcomere and contractile dysfunction (Nakai et al., 2007). Thus, we conclude that the abnormal sarcomere distribution in Vici-iPSC-CMs is attributed to the autophagy defect, which might lead to hypertrophic/dilated cardiomyopathy. To further confirm the phenotype, future study should be performed in even older Vici-iPSC-CMs or after the stress application. Additionally, the molecular level changes accompanied by activation of fetal genes such as atrial natriuretic factor and beta-myosin heavy chain should be clarified.

\subsubsection{The autophagic flux is blocked in Vici-iPSC-CMs}

Through analyzing the expression and localization of LC3, P62, NBR1 and LAMP1, we found the block of autophagic flux in Vici-iPSC-CMs, as indicated by the accumulation of the LC3II-positive autophagosomes and by the impaired fusion of autophagosomes with lysosomes.

Protein quality and quantity control rely on the proteasomes and lysosomes that are responsible for protein degradation in UPS and autophagy. However, it is still unknown how the different protein lytic systems including lysosomes, proteasomes and other protease are orchestrated to maintain protein stasis in various cell types under the physiological or pathological conditions (Wang and Robbins, 2014). Mostly, the naive and misfolded proteins are ubiquitinated with a cascade of enzymatic reactions that are 
catalyzed sequentially by E1 (ubiquitin-activating enzyme), E2 (ubiquitin-conjugating enzyme), and E3 (ubiquitin ligase). The aggregated proteins are degraded with the autophagy pathway while the organelles such as mitochondria and ribosomes tend to choose the selective autophagy through P62 and NBR1. But it has not been studied in this study whether Vici-iPSC-CMs causes the compensatory reaction from UPS and other proteolysis machinery.

The accumulation of autophagosomes and abnormal autolysosome formation in Vici-iPSC-CMs were also observed in Vici fibroblasts and skeletal muscle biopsy (Cullup et al. 2013) and lysosomal disorder disease (LSD), such as X-linked recessive Danon disease resulting from the mutation of LAMP2 (Nishino et al., 2000). Abnormal autophagosome accumulation was also visible in other LSD diseases such as Pompe disease, multiple sulfatase deficiency, mucopoly-saccharidosis type IIIA, mucolipidosis type IV and Gaucher disease (Curcio-Morelli et al., 2010; Fraldi et al., 2010; Sun et al., 2010; Tessitore et al., 2009; Venugopal et al., 2009; Vergarajauregui et al., 2008). Furthermore, the observation of the perinuclear localization of lysosomes in Vici-iPSC-CMs is also in line with those observed in LSD, in which the autophagosome accumulation is caused by a defect of lysosome biogenesis. These similar autophagy abnormalities in both LSD and Vici syndrome suggest that these disorders may intricately link to the same or related molecular pathways, which are perhaps associated with defects concerning intracellular trafficking or lysosome transport. Further investigation of EPG5 function such as its interaction proteins could be beneficial to uncover the molecular mechanism for the autophagosome accumulation and lysosome biogenesis defect in these diseases.

The disrupted autophagy could result in the accumulation of dysfunctional mitochondria that generate reactive oxygen species (ROS), which play a major role in the development of cardiovascular diseases (Linton et al., 2015). In this study, similar localization of COXIV-positive mitochondrial membranes between Ctr-iPSC-CMs and Vici-iPSC-CMs was observed. In order to assess if the autophagy defect causes mitochondrial dysfunction, 
better markers such as oxygen consumption rate, extracellular acidification rate, ATP production and respiratory capacity of mitochondria could serve as better indicators.

Collectively, we found in this study the accumulation of autophagosomes, the fusion impairment of autophagosomes with lysosomes as well as the abnormal perinuclear localization of lysosomes in Vici-iPSC-CMs.

\subsubsection{The PI3K/AKT/mTORC1 pathway is involved in the autophagy defect in Vici-iPSC-CMs}

In this study, decreased phosphorylation of AKT at two different activating sites T308 and S473 was found in Vici-iPSC-CMs. The major regulation pathway of autophagy is $\mathrm{PI} 3 \mathrm{~K} / \mathrm{AKT} / \mathrm{mTORC} 1$. Full activation of AKT results in phosphorylation of other proteins and mediates numerous cellular functions including cell growth, survival and apoptosis, protein synthesis, and cell metabolism. Aberrant regulation of phosphorylation of AKT is implicated in a number of human diseases including cancer, diabetes and cardiovascular diseases. For instance, the phosphorylation of AKT in cancer and human tumors is abnormally increased (Testa and Tsichlis, 2005). However, the relationship between the autophagy defect caused by EPG5 truncation and the reduced AKT phosphorylation in Vici-iPSC-CMs is still unknown. AKT activity is regulated by a plethora of proteins to keep in balance (Figure 43). PDK1 at the membrane is responsible for phosphorylation of AKT at T308 leading to partial activation of AKT (Andjelkovic et al., 1996). Further phosphorylation of AKT at S473 by mTORC2 stimulates its full enzymatic activity (Guertin et al., 2006). Members of the PI3K-related kinase (PIKK) family such as DNA-dependent protein kinase (DNA-PK) can also phosphorylate AKT at S473 (Feng et al., 2004). To avoid irregular phosphorylation of AKT, protein phosphatase 2A (PP2A), the PH-domain leucine-rich-repeat-containing protein phosphatases (PHLPP1/2) and the tumor suppressor PTEN dephosphorylate AKT to inhibit AKT activity (Andjelkovic et al., 1996; Brognard et al., 2007; Stambolic et al., 1998). Investigation of upstream regulatory proteins of AKT would be useful for studying the molecular mechanism of Vici syndrome associated cardiomyopathy. 


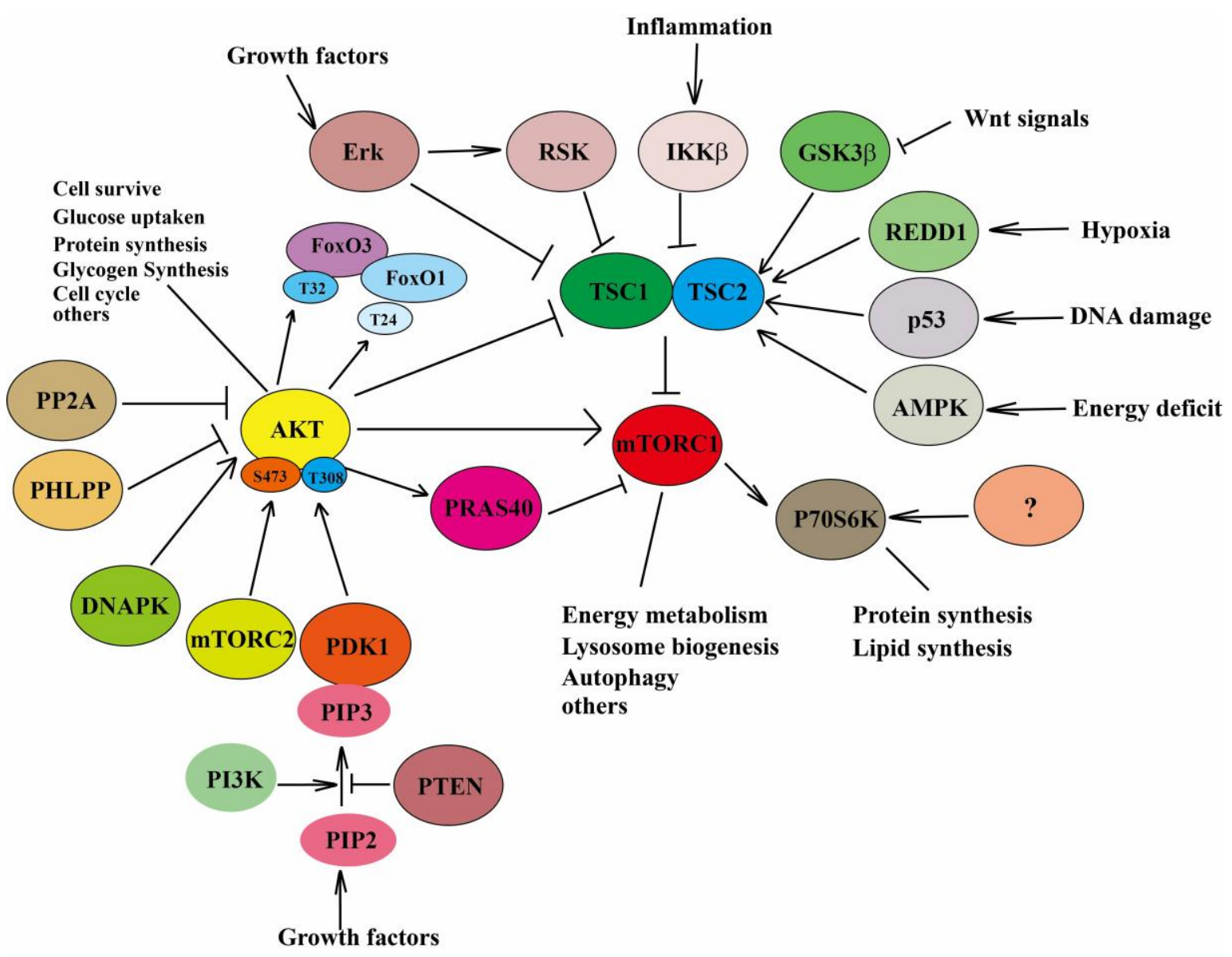

Figure 43. The schematic representation of partial AKT and mTORC1 cellular pathways. Both AKT and mTORC1 pathways are controlled by multiple upstream proteins, and regulate many downstream proteins to monitor the biological processes.

The downstream proteins of AKT, FOXO3 T32 and FOXO1 T24, showed reduced phosphorylation in Vici-iPSC-CMs, which thus may promote the function of active FoxO to activate gene expression that regulates cell death, cell cycle arrest and ROS production (Brunet et al., 1999; Gopinath et al., 2014; Myatt and Lam, 2007; Skurk et al., 2004). Expression of active FOXO3 can also directly induce autophagy and UPS in muscle cells (Mannis, 1987; Sippel, 1972) and induce expression of a number of autophagy-related genes including LC3 (Ravikumar et al. 2010). These data are in line with the upregulation of LC3 expression in Vici-iPSC-CMs. AKT also phosphorylates and inactivates the TSC1/TSC2 complex that negatively regulates mTORC1 to inhibit autophagy (Inoki et al., 2002). In this study, the decreased phosphorylation of mTOR at S2448 (activating site) was detected in Vici-iPSC-CMs, suggesting that the autophagy might be enhanced in Vici-iPSC-CMS. In addition, the reduced phosphorylation of mTOR perhaps leads to the reduced phosphorylation of AKT S473 that is directly activated by mTORC2. 
The activity of mTORC 1 is controlled by its upstream protein TSC1/2 complex that is regulated by multiple upstream signals (Figure 43). The effector kinases of Ras pathway Erk1/2 (extracellular-signal-regulated kinase1/2) and RSK1 (ribosomal S6 kinase) directly phosphorylate and inactivate the TSC1/2 complex and thus activate mTORC1 (Ma et al., 2005; Manning et al., 2002). The pro-inflammatory cytokines such as tumor necrosis factor- $\alpha(\mathrm{TNF} \alpha)$ activate mTORC1 similar to AKT, Erk1/2, RSK1 and IkB kinase (IKK $\beta$ ) by phosphorylating TSC1/2 to inhibit their function (Inoki et al., 2002; Lee et al., 2007). The Wnt pathway also activates mTORC1 by inhibiting GSK3 $\beta$ to phosphorylate TSC2 and promote TSC1/2 activity (Inoki et al., 2006). The preliminary analysis of the expression of total and phosphorylated GSK3 $\beta$ showed no significant differences in Vici-iPSC-CMs compared to Ctr-iPSC-CMs, suggesting that the Wnt signaling pathway does not influence the defective autophagy in Vici-iPSC-CMs. Furthermore, in hypoxia, energy deficit and DNA damage condition, TSC2 is phosphorylated to activate TSC1/2 through a cascade of proteins including DNA damage response 1 (REDD1), AMPK and P53, respectively, which negatively regulate mTORC1 (Brugarolas et al., 2004; Budanov and Karin, 2008; Feng et al., 2005; O'Brien and Elixson, 1990; Reiling and Hafen, 2004; Stambolic et al., 2001). Taken together, autophagy is regulated by mTORC1 but mTORC1 has a diverse function in protein synthesis, lipid synthesis, lysosome biogenesis, and energy metabolism (Figure 43).

In this study, rapamycin and bafilomycin are used for the induction and blockade of autophagy, respectively. Rapamycin treatment was supposed to inhibit mTORC1 efficiently, which theoretically suppresses the phosphorylation of P70S6K. But the phosphorylation of P70S6K was not changed after rapamycin and combined rapamycin and bafilomycin treatment in both Ctr- and Vici-iPSC-CMs. In addition, Expression and phosphorylation of P70S6K in untreated Vici-iPSC-CMs was similar to Ctr-iPSC-CMs. The unchanged phosphorylation of P70S6K in Vici-iPSC-CMs suggests that P70S6K might also be regulated by other mTOR-independent signaling.

Besides the main PI3K/AKT/mTOR pathway to regulate autophagy, other 
mTORC1-independent pathways are also involved. For example, second messenger IP3 (inositol 1,4,5-trisphosphate), cAMP-Epac-PLC- $\varepsilon$-IP3 and $\mathrm{Ca}^{2+}$-calpain-Gs $\alpha$ pathways negatively regulate autophagy (Sarkar et al., 2005; Sarkar et al., 2009; Williams et al., 2008). In the future, these pathways need to be investigated in Vici-iPSC-CMs.

\subsection{No isogenic control of Vici-iPSCs has been generated}

In this study, the CRISPR/Cas9 technology was applied to correct the EPG5 mutation. However, none of the 150 selected colonies possessed the "corrected" EPG5 gene. To rectify the mutation, the methods could be further modified in the future as following: (1) the oligonucleotides could be redesigned with appropriate length of homologous arms or the donor construct instead of single-stranded oligonucleotides should be designed; (2) the culture medium should be supplemented with a compound such as L755507 and Brefeldin A that could improve the efficiency of homology-directed repair (Yu et al., 2015); (3) the double-nicking approach should be applied to improve Cas9 targeting recognition fidelity through "sticky ends" generated by DNA single-stranded breaks (Ran et al., 2013), which are repaired via the high-fidelity base excision repair pathway to increase the specificity of NHEJ as well as homologous recombination. Recently, Natronobacterium gregoryi Argonaute as a DNA-guided endonuclease was discovered suitable for genome editing in human cells (Gao et al., 2016), which might be applied in the future to correct the EPG5 mutation.

Specificity of CRISPR/Cas9/sgRNA is affected by several factors, including the number, position and distribution of the mismatches in speculated off-target sites, enzymatic concentration of Cas9 as well as duration of Cas9 expression (Fu et al., 2013; Hsu et al., 2013; Mali et al., 2013a; Pattanayak et al., 2013). Duration of Cas9 expression after transduction to cells remains to be carefully investigated. In addition, whether the predicted off-target sites were recognized and cut by Cas9, it should be investigated further with whole genome sequencing or other unbiased ways of labeling DNA double strand break (Pattanayak et al., 2013, Hsu et al., 2014). 
In this study, the plasmid of CRISPR/Cas9 was linked with GFP to conveniently sort transfected iPSCs. The plasmids were easily transfected into HEK293T with high efficiency (50\%-70\%, with strong green fluorescence) as compared to Vici-iPSCs (10\%-15\%, with weak green fluorescence). Low green fluorescence signals were observed in Vici-iPSCs, which could be attributed to the silencing of CMV promoter of GFP. Therefore, to allow better sorting of GFP-positive Vici-iPSCs, control of GFP expression under a strong promoter such as EF-1alpha could be employed.

\subsection{Possible treatment of Vici syndrome}

Vici syndrome is the first multisystem disorder with a primary autophagy defect reported until now. Autophagy as a secondary phenotype has been implicated in many diseases such as cancer, neurodegeneration diseases, infectious diseases, myopathies and metabolic diseases. Therefore, autophagy modulation could be a potential therapeutic target for a wide range of diseases. Briefly, the diseases might be treated by upregulation or inhibition of autophagy due to its protective or destructive role in the progression of the disease. For instance, defects in autophagosome formation may be susceptible to enhance the autophagosome biogenesis; autophagy should be induced in infectious diseases to degrade the intracellular bacterial infections; autophagy should be inhibited in cancer cells as a tumor suppressor to prevent tumorgenesis.

The known pharmacological methods of modifying autophagy receive currently considerable attention (Rubinsztein et al., 2012; Yang et al., 2011). The drugs for induction of autophagy include mTROC1 inhibitor rapamycin and its analogues such as CCI-779, RAD001 and AP23573; mTOR kinase inhibitors including Torin1 and PP242; or mTOR independent drugs clonidine, rilmenidine, carbamazepine, trehalose, and Tat-beclin1 etc. Autophagy inhibitors consist of lysosomotropic inhibitors such as chloroquine, hydroxychloroquine, bafilomycin A1, nocodazole, and Lys05, autophagic machinery inhibitors such as 3-methyladenine and its derivatives PIK3C3 inhibitors, ATG4B inhibitors and ATG7 inhibitors etc (Huang and Klionsky, 2007). However, many of these drugs are still not applicable for clinical use. Currently, cancer is the first disease 
target for clinical trials that utilize the inhibition of autophagy in patients. Lysosomal inhibitor chloroquine and hydroxychloroquine that are applied to cure malaria are used in first round of Phase I/II clinical trials in cancer (Amaravadi et al., 2011; Yang et al., 2011). Phase I/II clinical trials showed promising results that hydroxychloroquine combined with chemotherapy and radiation therapy can stabilize aggressive cancers such as glioblastoma, melanoma, lymphoma and myeloma, renal and colon cancers. Indeed, to overcome the resistance to existing mTOR inhibitors, advanced mTOR inhibitor such as RapaLink-1 was explored recently and is prospective for treating the resistant tumors (Zhao et al., 2016).

Inhibition or induction of autophagy would be useful for studying cancer and neurodegenerative diseases; however, this method will not work for treating the diseases with defective maturation of autophagosomes and autolysosomes, such as Vici syndrome and lysosomal disorder. Therefore, further characterization of the precise biological role of EPG5 and finding the downstream protein as well as drug screening for promoting the fusion of autophagosomes with lysosomes will provide potential targets to treat Vici syndrome.

\subsection{Conclusion and outlook}

In conclusion, the findings of this study suggest that patient-specific iPSCs could be used to understand the function of autophagy in cardiomyocytes. Vici-iPSCs were generated from the patient with Vici syndrome carrying the EPG5 mutation. Vici-iPSCs were pluripotent and could be differentiated into functional beating cardiomyocytes. Furthermore, Vici-iPSC-CMs possessed disorganized sarcomere structure, accumulated autophagosomes, perinuclear lysosomes, blocked autophagosome-lysosome fusion and downregulated AKT activity. The data demonstrate that hiPSC-CMs are suitable to model the early onset disease such as Vici syndrome even though they resemble immature cardiomyocytes.

In the future, the EPG5 mutation may be rectified by genomic editing tools to rescue the 
disease phenotype observed in Vici-iPSC-CMs. Besides the analysis of sarcomere structure, functional contractility and autophagy defect, it is not known whether autophagy defect causes abnormal cardiac electrophysiological features such as action potential, excitation-contraction coupling and functional defect of ion channels. It is important to discover the precise biological role of EPG5 in physiological or pathological conditions and to elucidate mechanism of the autophagy defect in inherited multisystem disorder. To discover the molecular mechanism of Vici syndrome, the physiological function of EPG5 such as interaction proteins, cellular trafficking pathway and lysosome biogenesis should be figured out. Vici-iPSCs with primary autophagy defect also provide a platform for discovering the important role of autophagy in various tissues such as hepatocytes and neurons. 


\section{References}

Al-Owain, M., Al-Hashem, A., Al-Muhaizea, M., Humaidan, H., Al-Hindi, H., Al-Homoud, I., and Al-Mogarri, I. (2010). Vici syndrome associated with unilateral lung hypoplasia and myopathy. Am J Med Genet A 152A, 1849-1853.

Amaravadi, R.K., Lippincott-Schwartz, J., Yin, X.M., Weiss, W.A., Takebe, N., Timmer, W., DiPaola, R.S., Lotze, M.T., and White, E. (2011). Principles and current strategies for targeting autophagy for cancer treatment. Clin Cancer Res 17, 654-666.

American Heart, A. (2006). 2005 American Heart Association (AHA) guidelines for cardiopulmonary resuscitation (CPR) and emergency cardiovascular care (ECC) of pediatric and neonatal patients: pediatric basic life support. Pediatrics 117, e989-1004.

Andjelkovic, M., Jakubowicz, T., Cron, P., Ming, X.F., Han, J.W., and Hemmings, B.A. (1996). Activation and phosphorylation of a pleckstrin homology domain containing protein kinase (RAC-PK/PKB) promoted by serum and protein phosphatase inhibitors. Proc Natl Acad Sci U S A $93,5699-5704$.

Antonopoulos, A., Nikolopoulos, D., Georgiou, E.K., Kyriakidis, M., and Proukakis, C. (2002). Blood pressure elevation after phenylephrine infusion may adversely affect myocardial perfusion in patients with coronary artery disease. International journal of cardiology 84, 201-209.

Avior, Y., Sagi, I., and Benvenisty, N. (2016). Pluripotent stem cells in disease modelling and drug discovery. Nat Rev Mol Cell Biol 17, 170-182.

Awad, O., Sarkar, C., Panicker, L.M., Miller, D., Zeng, X., Sgambato, J.A., Lipinski, M.M., and Feldman, R.A. (2015). Altered TFEB-mediated lysosomal biogenesis in Gaucher disease iPSC-derived neuronal cells. Hum Mol Genet 24, 5775-5788.

Barmada, S.J., Serio, A., Arjun, A., Bilican, B., Daub, A., Ando, D.M., Tsvetkov, A., Pleiss, M., Li, X., Peisach, D., et al. (2014). Autophagy induction enhances TDP43 turnover and survival in neuronal ALS models. Nat Chem Biol 10, 677-685.

Bibikova, M., Beumer, K., Trautman, J.K., and Carroll, D. (2003). Enhancing gene targeting with designed zinc finger nucleases. Science 300, 764.

Bibikova, M., Golic, M., Golic, K.G., and Carroll, D. (2002). Targeted chromosomal cleavage and mutagenesis in Drosophila using zinc-finger nucleases. Genetics 161, 1169-1175.

Bjorkoy, G., Lamark, T., Brech, A., Outzen, H., Perander, M., Overvatn, A., Stenmark, H., and Johansen, T. (2005). p62/SQSTM1 forms protein aggregates degraded by autophagy and has a protective effect on huntingtin-induced cell death. J Cell Biol 171, 603-614.

Boch, J., Scholze, H., Schornack, S., Landgraf, A., Hahn, S., Kay, S., Lahaye, T., Nickstadt, A., 
and Bonas, U. (2009). Breaking the code of DNA binding specificity of TAL-type III effectors. Science 326, 1509-1512.

Bongso, A., Fong, C.Y., Ng, S.C., and Ratnam, S. (1994). Isolation and culture of inner cell mass cells from human blastocysts. Hum Reprod 9, 2110-2117.

Boya, P., Reggiori, F., and Codogno, P. (2013). Emerging regulation and functions of autophagy. Nat Cell Biol 15, 713-720.

Brennand, K.J., Simone, A., Jou, J., Gelboin-Burkhart, C., Tran, N., Sangar, S., Li, Y., Mu, Y., Chen, G., Yu, D., et al. (2011). Modelling schizophrenia using human induced pluripotent stem cells. Nature 473, 221-225.

Brognard, J., Sierecki, E., Gao, T., and Newton, A.C. (2007). PHLPP and a second isoform, PHLPP2, differentially attenuate the amplitude of Akt signaling by regulating distinct Akt isoforms. Mol Cell 25, 917-931.

Brugarolas, J., Lei, K., Hurley, R.L., Manning, B.D., Reiling, J.H., Hafen, E., Witters, L.A., Ellisen, L.W., and Kaelin, W.G., Jr. (2004). Regulation of mTOR function in response to hypoxia by REDD1 and the TSC1/TSC2 tumor suppressor complex. Genes Dev 18, 2893-2904.

Brunet, A., Bonni, A., Zigmond, M.J., Lin, M.Z., Juo, P., Hu, L.S., Anderson, M.J., Arden, K.C., Blenis, J., and Greenberg, M.E. (1999). Akt promotes cell survival by phosphorylating and inhibiting a Forkhead transcription factor. Cell 96, 857-868.

Budanov, A.V., and Karin, M. (2008). p53 target genes sestrin1 and sestrin2 connect genotoxic stress and mTOR signaling. Cell 134, 451-460.

Byrne, S., Dionisi-Vici, C., Smith, L., Gautel, M., and Jungbluth, H. (2016a). Vici syndrome: a review. Orphanet J Rare Dis 11, 21.

Byrne, S., Jansen, L., JM, U.K.-I., Siddiqui, A., Lidov, H.G., Bodi, I., Smith, L., Mein, R., Cullup, T., Dionisi-Vici, C., et al. (2016b). EPG5-related Vici syndrome: a paradigm of neurodevelopmental disorders with defective autophagy. Brain : a journal of neurology 139, 765-781.

Cao, N., Huang, Y., Zheng, J., Spencer, C.I., Zhang, Y., Fu, J.D., Nie, B., Xie, M., Zhang, M., Wang, H., et al. (2016). Conversion of human fibroblasts into functional cardiomyocytes by small molecules. Science.

Cardenas, C., Miller, R.A., Smith, I., Bui, T., Molgo, J., Muller, M., Vais, H., Cheung, K.H., Yang, J., Parker, I., et al. (2010). Essential regulation of cell bioenergetics by constitutive InsP3 receptor $\mathrm{Ca} 2+$ transfer to mitochondria. Cell 142, 270-283.

Chen, G., Gulbranson, D.R., Hou, Z., Bolin, J.M., Ruotti, V., Probasco, M.D., Smuga-Otto, K., Howden, S.E., Diol, N.R., Propson, N.E., et al. (2011). Chemically defined conditions for human 
iPSC derivation and culture. Nature methods 8, 424-429.

Chiyonobu, T., Yoshihara, T., Fukushima, Y., Yamamoto, Y., Tsunamoto, K., Nishimura, Y., Ishida, H., Toda, T., and Kasubuchi, Y. (2002). Sister and brother with Vici syndrome: agenesis of the corpus callosum, albinism, and recurrent infections. Am J Med Genet 109, 61-66.

Cho, S.W., Kim, S., Kim, J.M., and Kim, J.S. (2013). Targeted genome engineering in human cells with the Cas9 RNA-guided endonuclease. Nat Biotechnol 31, 230-232.

Choi, K.D., Yu, J., Smuga-Otto, K., Salvagiotto, G., Rehrauer, W., Vodyanik, M., Thomson, J., and Slukvin, I. (2009). Hematopoietic and endothelial differentiation of human induced pluripotent stem cells. Stem Cells 27, 559-567.

Cong, L., Ran, F.A., Cox, D., Lin, S., Barretto, R., Habib, N., Hsu, P.D., Wu, X., Jiang, W., Marraffini, L.A., et al. (2013). Multiplex genome engineering using CRISPR/Cas systems. Science 339, 819-823.

Cooper, O., Seo, H., Andrabi, S., Guardia-Laguarta, C., Graziotto, J., Sundberg, M., McLean, J.R., Carrillo-Reid, L., Xie, Z., Osborn, T., et al. (2012). Pharmacological rescue of mitochondrial deficits in iPSC-derived neural cells from patients with familial Parkinson's disease. Sci Transl Med 4, 141ra190.

Criollo, A., Vicencio, J.M., Tasdemir, E., Maiuri, M.C., Lavandero, S., and Kroemer, G. (2007). The inositol trisphosphate receptor in the control of autophagy. Autophagy 3, 350-353.

Cuervo, A.M., and Wong, E. (2014). Chaperone-mediated autophagy: roles in disease and aging. Cell Res 24, 92-104.

Cullup, T., Dionisi-Vici, C., Kho, A.L., Yau, S., Mohammed, S., Gautel, M., and Jungbluth, H. (2014). Clinical utility gene card for: Vici Syndrome. Eur J Hum Genet 22.

Cullup, T., Kho, A.L., Dionisi-Vici, C., Brandmeier, B., Smith, F., Urry, Z., Simpson, M.A., Yau, S., Bertini, E., McClelland, V., et al. (2013). Recessive mutations in EPG5 cause Vici syndrome, a multisystem disorder with defective autophagy. Nat Genet 45, 83-87.

Curcio-Morelli, C., Charles, F.A., Micsenyi, M.C., Cao, Y., Venugopal, B., Browning, M.F., Dobrenis, K., Cotman, S.L., Walkley, S.U., and Slaugenhaupt, S.A. (2010). Macroautophagy is defective in mucolipin-1-deficient mouse neurons. Neurobiol Dis 40, 370-377.

Daitoku, H., Sakamaki, J., and Fukamizu, A. (2011). Regulation of FoxO transcription factors by acetylation and protein-protein interactions. Biochimica et biophysica acta 1813, 1954-1960.

Davis, H.E., Morgan, J.R., and Yarmush, M.L. (2002). Polybrene increases retrovirus gene transfer efficiency by enhancing receptor-independent virus adsorption on target cell membranes. Biophys Chem 97, 159-172.

Davis, R.L., Weintraub, H., and Lassar, A.B. (1987). Expression of a single transfected cDNA 
converts fibroblasts to myoblasts. Cell 51, 987-1000.

Davis, R.P., Casini, S., van den Berg, C.W., Hoekstra, M., Remme, C.A., Dambrot, C., Salvatori, D., Oostwaard, D.W., Wilde, A.A., Bezzina, C.R., et al. (2012). Cardiomyocytes derived from pluripotent stem cells recapitulate electrophysiological characteristics of an overlap syndrome of cardiac sodium channel disease. Circulation 125, 3079-3091.

De Duve, C., Pressman, B.C., Gianetto, R., Wattiaux, R., and Appelmans, F. (1955). Tissue fractionation studies. 6. Intracellular distribution patterns of enzymes in rat-liver tissue. Biochem J 60, 604-617.

De Duve, C., and Wattiaux, R. (1966). Functions of lysosomes. Annu Rev Physiol 28, 435-492.

del Campo, M., Hall, B.D., Aeby, A., Nassogne, M.C., Verloes, A., Roche, C., Gonzalez, C., Sanchez, H., Garcia-Alix, A., Cabanas, F., et al. (1999). Albinism and agenesis of the corpus callosum with profound developmental delay: Vici syndrome, evidence for autosomal recessive inheritance. Am J Med Genet 85, 479-485.

DeRosa, B.A., Van Baaren, J.M., Dubey, G.K., Lee, J.M., Cuccaro, M.L., Vance, J.M., Pericak-Vance, M.A., and Dykxhoorn, D.M. (2012). Derivation of autism spectrum disorder-specific induced pluripotent stem cells from peripheral blood mononuclear cells. Neurosci Lett 516, 9-14.

Devine, M.J., Ryten, M., Vodicka, P., Thomson, A.J., Burdon, T., Houlden, H., Cavaleri, F., Nagano, M., Drummond, N.J., Taanman, J.W., et al. (2011). Parkinson's disease induced pluripotent stem cells with triplication of the alpha-synuclein locus. Nat Commun 2, 440.

DiCarlo, J.E., Norville, J.E., Mali, P., Rios, X., Aach, J., and Church, G.M. (2013). Genome engineering in Saccharomyces cerevisiae using CRISPR-Cas systems. Nucleic Acids Res 41, 4336-4343.

Dimos, J.T., Rodolfa, K.T., Niakan, K.K., Weisenthal, L.M., Mitsumoto, H., Chung, W., Croft, G.F., Saphier, G., Leibel, R., Goland, R., et al. (2008). Induced pluripotent stem cells generated from patients with ALS can be differentiated into motor neurons. Science 321, 1218-1221.

Dionisi Vici, C., Sabetta, G., Gambarara, M., Vigevano, F., Bertini, E., Boldrini, R., Parisi, S.G., Quinti, I., Aiuti, F., and Fiorilli, M. (1988). Agenesis of the corpus callosum, combined immunodeficiency, bilateral cataract, and hypopigmentation in two brothers. Am J Med Genet 29, $1-8$.

Draper, J.S., Smith, K., Gokhale, P., Moore, H.D., Maltby, E., Johnson, J., Meisner, L., Zwaka, T.P., Thomson, J.A., and Andrews, P.W. (2004). Recurrent gain of chromosomes 17q and 12 in cultured human embryonic stem cells. Nat Biotechnol 22, 53-54.

Dudek, J., Cheng, I.F., Balleininger, M., Vaz, F.M., Streckfuss-Bomeke, K., Hubscher, D., Vukotic, M., Wanders, R.J., Rehling, P., and Guan, K. (2013). Cardiolipin deficiency affects 
respiratory chain function and organization in an induced pluripotent stem cell model of Barth syndrome. Stem Cell Res 11, 806-819.

Dudek, J., Cheng, I.F., Chowdhury, A., Wozny, K., Balleininger, M., Reinhold, R., Grunau, S., Callegari, S., Toischer, K., Wanders, R.J., et al. (2016). Cardiac-specific succinate dehydrogenase deficiency in Barth syndrome. EMBO Mol Med 8, 139-154.

Eder, A., Vollert, I., Hansen, A., and Eschenhagen, T. (2016). Human engineered heart tissue as a model system for drug testing. Adv Drug Deliv Rev 96, 214-224.

Edgar, B.A. (2006). How flies get their size: genetics meets physiology. Nat Rev Genet 7, 907-916.

Ehmke, N., Parvaneh, N., Krawitz, P., Ashrafi, M.R., Karimi, P., Mehdizadeh, M., Kruger, U., Hecht, J., Mundlos, S., and Robinson, P.N. (2014). First description of a patient with Vici syndrome due to a mutation affecting the penultimate exon of EPG5 and review of the literature. Am J Med Genet A 164A, 3170-3175.

Eiges, R., Urbach, A., Malcov, M., Frumkin, T., Schwartz, T., Amit, A., Yaron, Y., Eden, A., Yanuka, O., Benvenisty, N., et al. (2007). Developmental study of fragile X syndrome using human embryonic stem cells derived from preimplantation genetically diagnosed embryos. Cell Stem Cell 1, 568-577.

El-Kersh, K., Jungbluth, H., Gringras, P., and Senthilvel, E. (2015). Severe Central Sleep Apnea in Vici Syndrome. Pediatrics 136, e1390-1394.

Esteban, M.A., Xu, J., Yang, J., Peng, M., Qin, D., Li, W., Jiang, Z., Chen, J., Deng, K., Zhong, M., et al. (2009). Generation of induced pluripotent stem cell lines from Tibetan miniature pig. J Biol Chem 284, 17634-17640.

Evans, M.J., and Kaufman, M.H. (1981). Establishment in culture of pluripotential cells from mouse embryos. Nature 292, 154-156.

Ezaki, J., Matsumoto, N., Takeda-Ezaki, M., Komatsu, M., Takahashi, K., Hiraoka, Y., Taka, H., Fujimura, T., Takehana, K., Yoshida, M., et al. (2011). Liver autophagy contributes to the maintenance of blood glucose and amino acid levels. Autophagy 7, 727-736.

Feng, J., Park, J., Cron, P., Hess, D., and Hemmings, B.A. (2004). Identification of a PKB/Akt hydrophobic motif Ser-473 kinase as DNA-dependent protein kinase. J Biol Chem 279, 41189-41196.

Feng, Z., Zhang, H., Levine, A.J., and Jin, S. (2005). The coordinate regulation of the p53 and mTOR pathways in cells. Proc Natl Acad Sci U S A 102, 8204-8209.

Filloux, F.M., Hoffman, R.O., Viskochil, D.H., Jungbluth, H., and Creel, D.J. (2014). Ophthalmologic features of Vici syndrome. J Pediatr Ophthalmol Strabismus 51, 214-220. 
Finocchi, A., Angelino, G., Cantarutti, N., Corbari, M., Bevivino, E., Cascioli, S., Randisi, F., Bertini, E., and Dionisi-Vici, C. (2012). Immunodeficiency in Vici syndrome: a heterogeneous phenotype. Am J Med Genet A 158A, 434-439.

Fraldi, A., Annunziata, F., Lombardi, A., Kaiser, H.J., Medina, D.L., Spampanato, C., Fedele, A.O., Polishchuk, R., Sorrentino, N.C., Simons, K., et al. (2010). Lysosomal fusion and SNARE function are impaired by cholesterol accumulation in lysosomal storage disorders. EMBO J 29, 3607-3620..

Frey, N., Katus, H.A., Olson, E.N., and Hill, J.A. (2004). Hypertrophy of the heart: a new therapeutic target? Circulation 109, 1580-1589.

Fu, Y., Foden, J.A., Khayter, C., Maeder, M.L., Reyon, D., Joung, J.K., and Sander, J.D. (2013). High-frequency off-target mutagenesis induced by CRISPR-Cas nucleases in human cells. Nat Biotechnol 31, 822-826.

Fusaki, N., Ban, H., Nishiyama, A., Saeki, K., and Hasegawa, M. (2009). Efficient induction of transgene-free human pluripotent stem cells using a vector based on Sendai virus, an RNA virus that does not integrate into the host genome. Proc Jpn Acad Ser B Phys Biol Sci 85, 348-362.

Gao, F., Shen, X.Z., Jiang, F., Wu, Y., and Han, C. (2016). DNA-guided genome editing using the Natronobacterium gregoryi Argonaute. Nat Biotechnol.

Gonzalez, F., Zhu, Z., Shi, Z.D., Lelli, K., Verma, N., Li, Q.V., and Huangfu, D. (2014). An iCRISPR platform for rapid, multiplexable, and inducible genome editing in human pluripotent stem cells. Cell Stem Cell 15, 215-226.

Gopinath, S.D., Webb, A.E., Brunet, A., and Rando, T.A. (2014). FOXO3 promotes quiescence in adult muscle stem cells during the process of self-renewal. Stem cell reports 2, 414-426.

Graef, M., and Nunnari, J. (2011). Mitochondria regulate autophagy by conserved signalling pathways. EMBO J 30, 2101-2114.

Gratz, S.J., Cummings, A.M., Nguyen, J.N., Hamm, D.C., Donohue, L.K., Harrison, M.M., Wildonger, J., and O'Connor-Giles, K.M. (2013). Genome engineering of Drosophila with the CRISPR RNA-guided Cas9 nuclease. Genetics 194, 1029-1035.

Grskovic, M., Javaherian, A., Strulovici, B., and Daley, G.Q. (2011). Induced pluripotent stem cells--opportunities for disease modelling and drug discovery. Nat Rev Drug Discov 10, 915-929.

Guertin, D.A., Stevens, D.M., Thoreen, C.C., Burds, A.A., Kalaany, N.Y., Moffat, J., Brown, M., Fitzgerald, K.J., and Sabatini, D.M. (2006). Ablation in mice of the mTORC components raptor, rictor, or mLST8 reveals that mTORC2 is required for signaling to Akt-FOXO and PKCalpha, but not S6K1. Dev Cell 11, 859-871.

Gurdon, J.B. (1962). The developmental capacity of nuclei taken from intestinal epithelium cells 
of feeding tadpoles. J Embryol Exp Morphol 10, 622-640.

Haack, T.B., Hogarth, P., Kruer, M.C., Gregory, A., Wieland, T., Schwarzmayr, T., Graf, E., Sanford, L., Meyer, E., Kara, E., et al. (2012). Exome sequencing reveals de novo WDR45 mutations causing a phenotypically distinct, X-linked dominant form of NBIA. Am J Hum Genet 91, 1144-1149.

Hershko, A., and Ciechanover, A. (1998). The ubiquitin system. Annu Rev Biochem 67, 425-479.

Hirano, M., Nakamura, Y., Saigoh, K., Sakamoto, H., Ueno, S., Isono, C., Miyamoto, K., Akamatsu, M., Mitsui, Y., and Kusunoki, S. (2013). Mutations in the gene encoding p62 in Japanese patients with amyotrophic lateral sclerosis. Neurology 80, 458-463.

Hochedlinger, K., and Jaenisch, R. (2002). Monoclonal mice generated by nuclear transfer from mature B and T donor cells. Nature 415, 1035-1038.

Honda, A., Hirose, M., Hatori, M., Matoba, S., Miyoshi, H., Inoue, K., and Ogura, A. (2010). Generation of induced pluripotent stem cells in rabbits: potential experimental models for human regenerative medicine. J Biol Chem 285, 31362-31369.

Hou, P., Li, Y., Zhang, X., Liu, C., Guan, J., Li, H., Zhao, T., Ye, J., Yang, W., Liu, K., et al. (2013). Pluripotent stem cells induced from mouse somatic cells by small-molecule compounds. Science 341, 651-654.

Hsu, P.D., Lander, E.S., and Zhang, F. (2014). Development and applications of CRISPR-Cas9 for genome engineering. Cell 157, 1262-1278.

Hsu, P.D., Scott, D.A., Weinstein, J.A., Ran, F.A., Konermann, S., Agarwala, V., Li, Y., Fine, E.J., Wu, X., Shalem, O., et al. (2013). DNA targeting specificity of RNA-guided Cas9 nucleases. Nat Biotechnol 31, 827-832.

Hu, B.Y., Weick, J.P., Yu, J., Ma, L.X., Zhang, X.Q., Thomson, J.A., and Zhang, S.C. (2010). Neural differentiation of human induced pluripotent stem cells follows developmental principles but with variable potency. Proc Natl Acad Sci U S A 107, 4335-4340.

Huang, J., and Klionsky, D.J. (2007). Autophagy and human disease. Cell Cycle 6, 1837-1849.

Huenerberg, K., Hudspeth, M., Bergmann, S., Pai, S., Singh, B., and Duong, A. (2016). Two cases of Vici syndrome associated with Idiopathic Thrombocytopenic Purpura (ITP) with a review of the literature. Am J Med Genet A 170, 1343-1346.

Hwang, W.Y., Fu, Y., Reyon, D., Maeder, M.L., Tsai, S.Q., Sander, J.D., Peterson, R.T., Yeh, J.R., and Joung, J.K. (2013). Efficient genome editing in zebrafish using a CRISPR-Cas system. Nat Biotechnol 31, 227-229.

Ieda, M., Fu, J.D., Delgado-Olguin, P., Vedantham, V., Hayashi, Y., Bruneau, B.G., and Srivastava, D. (2010). Direct reprogramming of fibroblasts into functional cardiomyocytes by defined factors. 
Cell 142, 375-386.

Inoki, K., Li, Y., Zhu, T., Wu, J., and Guan, K.L. (2002). TSC2 is phosphorylated and inhibited by Akt and suppresses mTOR signalling. Nat Cell Biol 4, 648-657.

Inoki, K., Ouyang, H., Zhu, T., Lindvall, C., Wang, Y., Zhang, X., Yang, Q., Bennett, C., Harada, Y., Stankunas, K., et al. (2006). TSC2 integrates Wnt and energy signals via a coordinated phosphorylation by AMPK and GSK3 to regulate cell growth. Cell 126, 955-968.

Itzhaki, I., Maizels, L., Huber, I., Zwi-Dantsis, L., Caspi, O., Winterstern, A., Feldman, O., Gepstein, A., Arbel, G., Hammerman, H., et al. (2011). Modelling the long QT syndrome with induced pluripotent stem cells. Nature 471, 225-U113.

Iwata, J., Ezaki, J., Komatsu, M., Yokota, S., Ueno, T., Tanida, I., Chiba, T., Tanaka, K., and Kominami, E. (2006). Excess peroxisomes are degraded by autophagic machinery in mammals. J Biol Chem 281, 4035-4041.

Jayawardena, T.M., Egemnazarov, B., Finch, E.A., Zhang, L., Payne, J.A., Pandya, K., Zhang, Z., Rosenberg, P., Mirotsou, M., and Dzau, V.J. (2012). MicroRNA-mediated in vitro and in vivo direct reprogramming of cardiac fibroblasts to cardiomyocytes. Circ Res 110, 1465-1473.

Kanamori, H., Takemura, G., Goto, K., Maruyama, R., Tsujimoto, A., Ogino, A., Takeyama, T., Kawaguchi, T., Watanabe, T., Fujiwara, T., et al. (2011). The role of autophagy emerging in postinfarction cardiac remodelling. Cardiovasc Res 91, 330-339.

Karantza-Wadsworth, V., Patel, S., Kravchuk, O., Chen, G., Mathew, R., Jin, S., and White, E. (2007). Autophagy mitigates metabolic stress and genome damage in mammary tumorigenesis. Genes Dev 21, 1621-1635.

Kaur, J., and Debnath, J. (2015). Autophagy at the crossroads of catabolism and anabolism. Nat Rev Mol Cell Biol 16, 461-472.

Kim, D., Kim, C.H., Moon, J.I., Chung, Y.G., Chang, M.Y., Han, B.S., Ko, S., Yang, E., Cha, K.Y., Lanza, R., et al. (2009a). Generation of human induced pluripotent stem cells by direct delivery of reprogramming proteins. Cell Stem Cell 4, 472-476.

Kim, J., Lengner, C.J., Kirak, O., Hanna, J., Cassady, J.P., Lodato, M.A., Wu, S., Faddah, D.A., Steine, E.J., Gao, Q., et al. (2011). Reprogramming of postnatal neurons into induced pluripotent stem cells by defined factors. Stem cells 29, 992-1000.

Kim, J.B., Sebastiano, V., Wu, G., Arauzo-Bravo, M.J., Sasse, P., Gentile, L., Ko, K., Ruau, D., Ehrich, M., van den Boom, D., et al. (2009b). Oct4-induced pluripotency in adult neural stem cells. Cell 136, 411-419.

Kirkin, V., Lamark, T., Sou, Y.S., Bjorkoy, G., Nunn, J.L., Bruun, J.A., Shvets, E., McEwan, D.G., Clausen, T.H., Wild, P., et al. (2009a). A role for NBR1 in autophagosomal degradation of 
ubiquitinated substrates. Mol Cell 33, 505-516.

Kirkin, V., McEwan, D.G., Novak, I., and Dikic, I. (2009b). A role for ubiquitin in selective autophagy. Mol Cell 34, 259-269.

Kitsis, R.N., Peng, C.F., and Cuervo, A.M. (2007). Eat your heart out. Nat Med 13, 539-541.

Klionsky, D.J., Abdalla, F.C., Abeliovich, H., Abraham, R.T., Acevedo-Arozena, A., Adeli, K., Agholme, L., Agnello, M., Agostinis, P., Aguirre-Ghiso, J.A., et al. (2012). Guidelines for the use and interpretation of assays for monitoring autophagy. Autophagy 8, 445-544.

Koivusalo, M., Kapus, A., and Grinstein, S. (2009). Sensors, transducers, and effectors that regulate cell size and shape. J Biol Chem 284, 6595-6599.

Komatsu, M., Waguri, S., Ueno, T., Iwata, J., Murata, S., Tanida, I., Ezaki, J., Mizushima, N., Ohsumi, Y., Uchiyama, Y., et al. (2005). Impairment of starvation-induced and constitutive autophagy in Atg7-deficient mice. J Cell Biol 169, 425-434.

Kuma, A., Hatano, M., Matsui, M., Yamamoto, A., Nakaya, H., Yoshimori, T., Ohsumi, Y., Tokuhisa, T., and Mizushima, N. (2004). The role of autophagy during the early neonatal starvation period. Nature 432, 1032-1036.

Laplante, M., and Sabatini, D.M. (2012). mTOR signaling in growth control and disease. Cell 149, 274-293.

Lee, D.F., Kuo, H.P., Chen, C.T., Hsu, J.M., Chou, C.K., Wei, Y., Sun, H.L., Li, L.Y., Ping, B., Huang, W.C., et al. (2007). IKK beta suppression of TSC1 links inflammation and tumor angiogenesis via the mTOR pathway. Cell 130,440-455.

Lee, G., Papapetrou, E.P., Kim, H., Chambers, S.M., Tomishima, M.J., Fasano, C.A., Ganat, Y.M., Menon, J., Shimizu, F., Viale, A., et al. (2009). Modelling pathogenesis and treatment of familial dysautonomia using patient-specific iPSCs. Nature 461, 402-406.

Levine, B., and Kroemer, G. (2008). Autophagy in the pathogenesis of disease. Cell 132, 27-42.

Li, W., Wei, W., Zhu, S., Zhu, J., Shi, Y., Lin, T., Hao, E., Hayek, A., Deng, H., and Ding, S. (2009). Generation of rat and human induced pluripotent stem cells by combining genetic reprogramming and chemical inhibitors. Cell Stem Cell 4, 16-19.

Li, W.W., Li, J., and Bao, J.K. (2012). Microautophagy: lesser-known self-eating. Cell Mol Life Sci 69, 1125-1136.

Lian, X., Hsiao, C., Wilson, G., Zhu, K., Hazeltine, L.B., Azarin, S.M., Raval, K.K., Zhang, J., Kamp, T.J., and Palecek, S.P. (2012). Robust cardiomyocyte differentiation from human pluripotent stem cells via temporal modulation of canonical Wnt signaling. Proc Natl Acad Sci U S A 109, E1848-1857. 
Liang, P., Lan, F., Lee, A.S., Gong, T., Sanchez-Freire, V., Wang, Y., Diecke, S., Sallam, K., Knowles, J.W., Wang, P.J., et al. (2013). Drug screening using a library of human induced pluripotent stem cell-derived cardiomyocytes reveals disease-specific patterns of cardiotoxicity. Circulation 127, 1677-1691.

Liang, X.H., Jackson, S., Seaman, M., Brown, K., Kempkes, B., Hibshoosh, H., and Levine, B. (1999). Induction of autophagy and inhibition of tumorigenesis by beclin 1. Nature 402, 672-676.

Liao, J., Cui, C., Chen, S., Ren, J., Chen, J., Gao, Y., Li, H., Jia, N., Cheng, L., Xiao, H., et al. (2009). Generation of induced pluripotent stem cell lines from adult rat cells. Cell Stem Cell 4, $11-15$.

Lieu, D.K., Liu, J., Siu, C.W., McNerney, G.P., Tse, H.F., Abu-Khalil, A., Huser, T., and Li, R.A. (2009). Absence of transverse tubules contributes to non-uniform $\mathrm{Ca}(2+)$ wavefronts in mouse and human embryonic stem cell-derived cardiomyocytes. Stem Cells Dev 18, 1493-1500.

Linton, P.J., Gurney, M., Sengstock, D., Mentzer, R.M., Jr., and Gottlieb, R.A. (2015). This old heart: Cardiac aging and autophagy. J Mol Cell Cardiol 83, 44-54.

Liu, H., Zhu, F., Yong, J., Zhang, P., Hou, P., Li, H., Jiang, W., Cai, J., Liu, M., Cui, K., et al. (2008). Generation of induced pluripotent stem cells from adult rhesus monkey fibroblasts. Cell Stem Cell 3, 587-590.

Liu, X., Qi, J., Xu, X., Zeisberg, M., Guan, K., and Zeisberg, E.M. (2016). Differentiation of functional endothelial cells from human induced pluripotent stem cells: A novel, highly efficient and cost effective method. Differentiation.

Lloyd, A.C. (2013). The regulation of cell size. Cell 154, 1194-1205.

Loffredo, F.S., Steinhauser, M.L., Jay, S.M., Gannon, J., Pancoast, J.R., Yalamanchi, P., Sinha, M., Dall'Osso, C., Khong, D., Shadrach, J.L., et al. (2013). Growth differentiation factor 11 is a circulating factor that reverses age-related cardiac hypertrophy. Cell 153, 828-839.

Loh, Y.H., Agarwal, S., Park, I.H., Urbach, A., Huo, H., Heffner, G.C., Kim, K., Miller, J.D., Ng, K., and Daley, G.Q. (2009). Generation of induced pluripotent stem cells from human blood. Blood 113, 5476-5479.

Lu, Q., Yokoyama, C.C., Williams, J.W., Baldridge, M.T., Jin, X., DesRochers, B., Bricker, T., Wilen, C.B., Bagaitkar, J., Loginicheva, E., et al. (2016). Homeostatic Control of Innate Lung Inflammation by Vici Syndrome Gene Epg5 and Additional Autophagy Genes Promotes Influenza Pathogenesis. Cell Host Microbe 19, 102-113.

Lundy, S.D., Zhu, W.Z., Regnier, M., and Laflamme, M.A. (2013). Structural and functional maturation of cardiomyocytes derived from human pluripotent stem cells. Stem Cells Dev 22, 1991-2002. 
Ma, L., Chen, Z., Erdjument-Bromage, H., Tempst, P., and Pandolfi, P.P. (2005). Phosphorylation and functional inactivation of TSC2 by Erk implications for tuberous sclerosis and cancer pathogenesis. Cell 121, 179-193.

Maiuri, M.C., Galluzzi, L., Morselli, E., Kepp, O., Malik, S.A., and Kroemer, G. (2010). Autophagy regulation by p53. Curr Opin Cell Biol 22, 181-185.

Mali, P., Aach, J., Stranges, P.B., Esvelt, K.M., Moosburner, M., Kosuri, S., Yang, L., and Church, G.M. (2013a). CAS9 transcriptional activators for target specificity screening and paired nickases for cooperative genome engineering. Nat Biotechnol 31, 833-838.

Mali, P., Yang, L., Esvelt, K.M., Aach, J., Guell, M., DiCarlo, J.E., Norville, J.E., and Church, G.M. (2013b). RNA-guided human genome engineering via Cas9. Science 339, 823-826.

Mannhardt, I., Breckwoldt, K., Letuffe-Breniere, D., Schaaf, S., Schulz, H., Neuber, C., Benzin, A., Werner, T., Eder, A., Schulze, T., et al. (2016). Human Engineered Heart Tissue: Analysis of Contractile Force. Stem cell reports 7, 29-42.

Manning, B.D., Tee, A.R., Logsdon, M.N., Blenis, J., and Cantley, L.C. (2002). Identification of the tuberous sclerosis complex-2 tumor suppressor gene product tuberin as a target of the phosphoinositide 3-kinase/akt pathway. Mol Cell 10, 151-162.

Mannis, M.J. (1987). Making sense of contrast sensitivity testing. Has its time come? Arch Ophthalmol 105, 627-629.

Marino, G., Niso-Santano, M., Baehrecke, E.H., and Kroemer, G. (2014). Self-consumption: the interplay of autophagy and apoptosis. Nat Rev Mol Cell Biol 15, 81-94.

Marino, G., Salvador-Montoliu, N., Fueyo, A., Knecht, E., Mizushima, N., and Lopez-Otin, C. (2007). Tissue-specific autophagy alterations and increased tumorigenesis in mice deficient in Atg4C/autophagin-3. J Biol Chem 282, 18573-18583.

Marson, A., Foreman, R., Chevalier, B., Bilodeau, S., Kahn, M., Young, R.A., and Jaenisch, R. (2008). Wnt signaling promotes reprogramming of somatic cells to pluripotency. Cell Stem Cell 3, 132-135.

Martins-Taylor, K., and Xu, R.H. (2012). Concise review: Genomic stability of human induced pluripotent stem cells. Stem cells 30, 22-27.

Mathur, A., Ma, Z., Loskill, P., Jeeawoody, S., and Healy, K.E. (2016). In vitro cardiac tissue models: Current status and future prospects. Adv Drug Deliv Rev 96, 203-213.

McClelland, V., Cullup, T., Bodi, I., Ruddy, D., Buj-Bello, A., Biancalana, V., Boehm, J., Bitoun, M., Miller, O., Jan, W., et al. (2010). Vici syndrome associated with sensorineural hearing loss and evidence of neuromuscular involvement on muscle biopsy. Am J Med Genet A 152A, 741-747. 
Miyata, R., Hayashi, M., and Itoh, E. (2014). Pathological changes in cardiac muscle and cerebellar cortex in Vici syndrome. Am J Med Genet A 164A, 3203-3205.

Miyata, R., Hayashi, M., Sato, H., Sugawara, Y., Yui, T., Araki, S., Hasegawa, T., Doi, S., and Kohyama, J. (2007). Sibling cases of Vici syndrome: sleep abnormalities and complications of renal tubular acidosis. Am J Med Genet A 143A, 189-194.

Morris, J.K., Wald, N.J., and Watt, H.C. (1999). Fetal loss in Down syndrome pregnancies. Prenat Diagn 19, 142-145.

Moscou, M.J., and Bogdanove, A.J. (2009). A simple cipher governs DNA recognition by TAL effectors. Science 326, 1501.

Myatt, S.S., and Lam, E.W. (2007). The emerging roles of forkhead box (Fox) proteins in cancer. Nat Rev Cancer 7, 847-859.

Nakai, A., Yamaguchi, O., Takeda, T., Higuchi, Y., Hikoso, S., Taniike, M., Omiya, S., Mizote, I., Matsumura, Y., Asahi, M., et al. (2007). The role of autophagy in cardiomyocytes in the basal state and in response to hemodynamic stress. Nat Med 13, 619-624.

Nelakanti, R.V., Kooreman, N.G., and Wu, J.C. (2015). Teratoma formation: a tool for monitoring pluripotency in stem cell research. Curr Protoc Stem Cell Biol 32, 4A 8 1-17.

Neufeld, T.P. (2010). TOR-dependent control of autophagy: biting the hand that feeds. Curr Opin Cell Biol 22, 157-168.

Nishida, K., Kyoi, S., Yamaguchi, O., Sadoshima, J., and Otsu, K. (2009). The role of autophagy in the heart. Cell Death Differ 16, 31-38.

Nishida, K., and Otsu, K. (2015). Autophagy during cardiac remodeling. J Mol Cell Cardiol.

Nishino, I., Fu, J., Tanji, K., Yamada, T., Shimojo, S., Koori, T., Mora, M., Riggs, J.E., Oh, S.J., Koga, Y., et al. (2000). Primary LAMP-2 deficiency causes X-linked vacuolar cardiomyopathy and myopathy (Danon disease). Nature 406, 906-910.

Nixon, R.A., Wegiel, J., Kumar, A., Yu, W.H., Peterhoff, C., Cataldo, A., and Cuervo, A.M. (2005). Extensive involvement of autophagy in Alzheimer disease: an immuno-electron microscopy study. J Neuropathol Exp Neurol 64, 113-122.

O'Brien, P., and Elixson, E.M. (1990). The child following the Fontan procedure: nursing strategies. AACN Clin Issues Crit Care Nurs 1, 46-58.

Okita, K., Matsumura, Y., Sato, Y., Okada, A., Morizane, A., Okamoto, S., Hong, H., Nakagawa, M., Tanabe, K., Tezuka, K., et al. (2011). A more efficient method to generate integration-free human iPS cells. Nat Methods 8, 409-412.

Okita, K., Nakagawa, M., Hyenjong, H., Ichisaka, T., and Yamanaka, S. (2008). Generation of 
mouse induced pluripotent stem cells without viral vectors. Science 322, 949-953.

Ozkale, M., Erol, I., Gumus, A., Ozkale, Y., and Alehan, F. (2012). Vici syndrome associated with sensorineural hearing loss and laryngomalacia. Pediatr Neurol 47, 375-378.

Pang, Z.P., Yang, N., Vierbuchen, T., Ostermeier, A., Fuentes, D.R., Yang, T.Q., Citri, A., Sebastiano, V., Marro, S., Sudhof, T.C., et al. (2011). Induction of human neuronal cells by defined transcription factors. Nature 476, 220-223.

Pattanayak, V., Lin, S., Guilinger, J.P., Ma, E., Doudna, J.A., and Liu, D.R. (2013). High-throughput profiling of off-target DNA cleavage reveals RNA-programmed Cas9 nuclease specificity. Nat Biotechnol 31, 839-843.

Pickford, F., Masliah, E., Britschgi, M., Lucin, K., Narasimhan, R., Jaeger, P.A., Small, S., Spencer, B., Rockenstein, E., Levine, B., et al. (2008). The autophagy-related protein beclin 1 shows reduced expression in early Alzheimer disease and regulates amyloid beta accumulation in mice. J Clin Invest 118, 2190-2199.

Przyborski, S.A. (2005). Differentiation of human embryonic stem cells after transplantation in immune-deficient mice. Stem cells 23, 1242-1250.

Pua, H.H., Dzhagalov, I., Chuck, M., Mizushima, N., and He, Y.W. (2007). A critical role for the autophagy gene Atg5 in T cell survival and proliferation. J Exp Med 204, 25-31.

Qian, L., Huang, Y., Spencer, C.I., Foley, A., Vedantham, V., Liu, L., Conway, S.J., Fu, J.D., and Srivastava, D. (2012). In vivo reprogramming of murine cardiac fibroblasts into induced cardiomyocytes. Nature 485, 593-598.

Qu, X., Zou, Z., Sun, Q., Luby-Phelps, K., Cheng, P., Hogan, R.N., Gilpin, C., and Levine, B. (2007). Autophagy gene-dependent clearance of apoptotic cells during embryonic development. Cell 128, 931-946.

Ran, F.A., Hsu, P.D., Lin, C.Y., Gootenberg, J.S., Konermann, S., Trevino, A.E., Scott, D.A., Inoue, A., Matoba, S., Zhang, Y., et al. (2013). Double nicking by RNA-guided CRISPR Cas9 for enhanced genome editing specificity. Cell 154, 1380-1389.

Raval, K.K., Tao, R., White, B.E., De Lange, W.J., Koonce, C.H., Yu, J., Kishnani, P.S., Thomson, J.A., Mosher, D.F., Ralphe, J.C., et al. (2015). Pompe disease results in a Golgi-based glycosylation deficit in human induced pluripotent stem cell-derived cardiomyocytes. J Biol Chem 290, 3121-3136.

Ravikumar, B., Sarkar, S., Davies, J.E., Futter, M., Garcia-Arencibia, M., Green-Thompson, Z.W., Jimenez-Sanchez, M., Korolchuk, V.I., Lichtenberg, M., Luo, S., et al. (2010). Regulation of mammalian autophagy in physiology and pathophysiology. Physiol Rev 90, 1383-1435.

Reiling, J.H., and Hafen, E. (2004). The hypoxia-induced paralogs Scylla and Charybdis inhibit 
growth by down-regulating S6K activity upstream of TSC in Drosophila. Genes Dev 18, 2879-2892.

Ren, Y., Jiang, H., Hu, Z., Fan, K., Wang, J., Janoschka, S., Wang, X., Ge, S., and Feng, J. (2015). Parkin mutations reduce the complexity of neuronal processes in iPSC-derived human neurons. Stem cells 33, 68-78.

Robertson, C., Tran, D.D., and George, S.C. (2013). Concise review: maturation phases of human pluripotent stem cell-derived cardiomyocytes. Stem cells 31, 829-837.

Rogers, R.C., Aufmuth, B., and Monesson, S. (2011). Vici syndrome: a rare autosomal recessive syndrome with brain anomalies, cardiomyopathy, and severe intellectual disability. Case Rep Genet 2011, 421582 .

Rubinsztein, D.C., Codogno, P., and Levine, B. (2012). Autophagy modulation as a potential therapeutic target for diverse diseases. Nat Rev Drug Discov 11, 709-730.

Ryan, K.M. (2011). p53 and autophagy in cancer: guardian of the genome meets guardian of the proteome. Eur J Cancer 47, 44-50.

Ryan, S.D., Dolatabadi, N., Chan, S.F., Zhang, X., Akhtar, M.W., Parker, J., Soldner, F., Sunico, C.R., Nagar, S., Talantova, M., et al. (2013). Isogenic human iPSC Parkinson's model shows nitrosative stress-induced dysfunction in MEF2-PGC1alpha transcription. Cell 155, 1351-1364.

Said, E., Soler, D., and Sewry, C. (2012). Vici syndrome--a rapidly progressive neurodegenerative disorder with hypopigmentation, immunodeficiency and myopathic changes on muscle biopsy. Am J Med Genet A 158A, 440-444.

Saitsu, H., Nishimura, T., Muramatsu, K., Kodera, H., Kumada, S., Sugai, K., Kasai-Yoshida, E., Sawaura, N., Nishida, H., Hoshino, A., et al. (2013). De novo mutations in the autophagy gene WDR45 cause static encephalopathy of childhood with neurodegeneration in adulthood. Nat Genet 45, 445-449, 449e441.

Sances, S., Bruijn, L.I., Chandran, S., Eggan, K., Ho, R., Klim, J.R., Livesey, M.R., Lowry, E., Macklis, J.D., Rushton, D., et al. (2016). Modeling ALS with motor neurons derived from human induced pluripotent stem cells. Nat Neurosci 16, 542-553.

Sarkar, S., Floto, R.A., Berger, Z., Imarisio, S., Cordenier, A., Pasco, M., Cook, L.J., and Rubinsztein, D.C. (2005). Lithium induces autophagy by inhibiting inositol monophosphatase. J Cell Biol 170, 1101-1111.

Sarkar, S., Ravikumar, B., Floto, R.A., and Rubinsztein, D.C. (2009). Rapamycin and mTOR-independent autophagy inducers ameliorate toxicity of polyglutamine-expanded huntingtin and related proteinopathies. Cell Death Differ 16, 46-56.

Sarkar, S., and Rubinsztein, D.C. (2006). Inositol and IP3 levels regulate autophagy: biology and 
therapeutic speculations. Autophagy 2, 132-134.

Schwank, G., Koo, B.K., Sasselli, V., Dekkers, J.F., Heo, I., Demircan, T., Sasaki, N., Boymans, S., Cuppen, E., van der Ent, C.K., et al. (2013). Functional repair of CFTR by CRISPR/Cas9 in intestinal stem cell organoids of cystic fibrosis patients. Cell Stem Cell 13, 653-658.

Shaid, S., Brandts, C.H., Serve, H., and Dikic, I. (2013). Ubiquitination and selective autophagy. Cell Death Differ 20, 21-30.

Shcheglovitov, A., Shcheglovitova, O., Yazawa, M., Portmann, T., Shu, R., Sebastiano, V., Krawisz, A., Froehlich, W., Bernstein, J.A., Hallmayer, J.F., et al. (2013). SHANK3 and IGF1 restore synaptic deficits in neurons from 22q13 deletion syndrome patients. Nature 503, 267-271.

Shimada, H., Nakada, A., Hashimoto, Y., Shigeno, K., Shionoya, Y., and Nakamura, T. (2010). Generation of canine induced pluripotent stem cells by retroviral transduction and chemical inhibitors. Mol Reprod Dev 77, 2.

Si-Tayeb, K., Noto, F.K., Sepac, A., Sedlic, F., Bosnjak, Z.J., Lough, J.W., and Duncan, S.A. (2010). Generation of human induced pluripotent stem cells by simple transient transfection of plasmid DNA encoding reprogramming factors. BMC Dev Biol 10, 81.

Simms-Waldrip, T., Rodriguez-Gonzalez, A., Lin, T., Ikeda, A.K., Fu, C., and Sakamoto, K.M. (2008). The aggresome pathway as a target for therapy in hematologic malignancies. Mol Genet Metab 94, 283-286.

Sippel, A. (1972). [Is the Gompertz's law of mortality to be replaced by a more accurate one?]. Z Naturforsch B 27, 456-460.

Skurk, C., Maatz, H., Kim, H.S., Yang, J., Abid, M.R., Aird, W.C., and Walsh, K. (2004). The Akt-regulated forkhead transcription factor FOXO3a controls endothelial cell viability through modulation of the caspase-8 inhibitor FLIP. J Biol Chem 279, 1513-1525.

Small, S.A., Kent, K., Pierce, A., Leung, C., Kang, M.S., Okada, H., Honig, L., Vonsattel, J.P., and Kim, T.W. (2005). Model-guided microarray implicates the retromer complex in Alzheimer's disease. Ann Neurol 58, 909-919.

Smith, A.G., Heath, J.K., Donaldson, D.D., Wong, G.G., Moreau, J., Stahl, M., and Rogers, D. (1988). Inhibition of pluripotential embryonic stem cell differentiation by purified polypeptides. Nature 336, 688-690.

Soga, M., Ishitsuka, Y., Hamasaki, M., Yoneda, K., Furuya, H., Matsuo, M., Ihn, H., Fusaki, N., Nakamura, K., Nakagata, N., et al. (2015). HPGCD outperforms HPBCD as a potential treatment for Niemann-Pick disease type C during disease modeling with iPS cells. Stem cells 33, 1075-1088.

Solter, D. (2006). From teratocarcinomas to embryonic stem cells and beyond: a history of 
embryonic stem cell research. Nat Rev Genet 7, 319-327.

Somers, A., Jean, J.C., Sommer, C.A., Omari, A., Ford, C.C., Mills, J.A., Ying, L., Sommer, A.G., Jean, J.M., Smith, B.W., et al. (2010). Generation of transgene-free lung disease-specific human induced pluripotent stem cells using a single excisable lentiviral stem cell cassette. Stem cells 28 , 1728-1740.

Song, K., Nam, Y.J., Luo, X., Qi, X., Tan, W., Huang, G.N., Acharya, A., Smith, C.L., Tallquist, M.D., Neilson, E.G., et al. (2012). Heart repair by reprogramming non-myocytes with cardiac transcription factors. Nature 485, 599-604.

Soong, P.L., Tiburcy, M., and Zimmermann, W.H. (2012). Cardiac differentiation of human embryonic stem cells and their assembly into engineered heart muscle. Curr Protoc Cell Biol Chapter 23, Unit23 28.

Spence, J.R., Mayhew, C.N., Rankin, S.A., Kuhar, M.F., Vallance, J.E., Tolle, K., Hoskins, E.E., Kalinichenko, V.V., Wells, S.I., Zorn, A.M., et al. (2011). Directed differentiation of human pluripotent stem cells into intestinal tissue in vitro. Nature 470, 105-109.

Stadtfeld, M., Nagaya, M., Utikal, J., Weir, G., and Hochedlinger, K. (2008). Induced pluripotent stem cells generated without viral integration. Science 322, 945-949.

Stambolic, V., MacPherson, D., Sas, D., Lin, Y., Snow, B., Jang, Y., Benchimol, S., and Mak, T.W. (2001). Regulation of PTEN transcription by p53. Mol Cell 8, 317-325.

Stambolic, V., Suzuki, A., de la Pompa, J.L., Brothers, G.M., Mirtsos, C., Sasaki, T., Ruland, J., Penninger, J.M., Siderovski, D.P., and Mak, T.W. (1998). Negative regulation of PKB/Akt-dependent cell survival by the tumor suppressor PTEN. Cell 95, 29-39.

Streckfuss-Bomeke, K., Wolf, F., Azizian, A., Stauske, M., Tiburcy, M., Wagner, S., Hubscher, D., Dressel, R., Chen, S., Jende, J., et al. (2013). Comparative study of human-induced pluripotent stem cells derived from bone marrow cells, hair keratinocytes, and skin fibroblasts. Eur Heart $\mathbf{J}$ 34, 2618-2629.

Sullivan, G.J., Hay, D.C., Park, I.H., Fletcher, J., Hannoun, Z., Payne, C.M., Dalgetty, D., Black, J.R., Ross, J.A., Samuel, K., et al. (2010). Generation of functional human hepatic endoderm from human induced pluripotent stem cells. Hepatology 51, 329-335.

Sun, Y., Liou, B., Ran, H., Skelton, M.R., Williams, M.T., Vorhees, C.V., Kitatani, K., Hannun, Y.A., Witte, D.P., Xu, Y.H., et al. (2010). Neuronopathic Gaucher disease in the mouse: viable combined selective saposin C deficiency and mutant glucocerebrosidase (V394L) mice with glucosylsphingosine and glucosylceramide accumulation and progressive neurological deficits. Hum Mol Genet 19, 1088-1097.

Tada, M., Takahama, Y., Abe, K., Nakatsuji, N., and Tada, T. (2001). Nuclear reprogramming of somatic cells by in vitro hybridization with ES cells. Curr Biol 11, 1553-1558. 
Takahashi, K., Tanabe, K., Ohnuki, M., Narita, M., Ichisaka, T., Tomoda, K., and Yamanaka, S. (2007). Induction of pluripotent stem cells from adult human fibroblasts by defined factors. Cell $131,861-872$.

Takahashi, K., and Yamanaka, S. (2006). Induction of pluripotent stem cells from mouse embryonic and adult fibroblast cultures by defined factors. Cell 126, 663-676.

Takebe, T., Sekine, K., Enomura, M., Koike, H., Kimura, M., Ogaeri, T., Zhang, R.R., Ueno, Y., Zheng, Y.W., Koike, N., et al. (2013). Vascularized and functional human liver from an iPSC-derived organ bud transplant. Nature 499, 481-484.

Tanaka, Y., Guhde, G., Suter, A., Eskelinen, E.L., Hartmann, D., Lullmann-Rauch, R., Janssen, P.M., Blanz, J., von Figura, K., and Saftig, P. (2000). Accumulation of autophagic vacuoles and cardiomyopathy in LAMP-2-deficient mice. Nature 406, 902-906.

Tannous, P., Zhu, H., Nemchenko, A., Berry, J.M., Johnstone, J.L., Shelton, J.M., Miller, F.J., Jr., Rothermel, B.A., and Hill, J.A. (2008). Intracellular protein aggregation is a proximal trigger of cardiomyocyte autophagy. Circulation 117, 3070-3078.

Tasdemir, S., Sahin, I., Cayir, A., Yuce, I., Ceylaner, S., and Tatar, A. (2016). Vici syndrome in siblings born to consanguineous parents. Am J Med Genet A 170, 220-225.

Terman, A., and Brunk, U.T. (2005). Autophagy in cardiac myocyte homeostasis, aging, and pathology. Cardiovasc Res 68, 355-365.

Tessitore, A., Pirozzi, M., and Auricchio, A. (2009). Abnormal autophagy, ubiquitination, inflammation and apoptosis are dependent upon lysosomal storage and are useful biomarkers of mucopolysaccharidosis VI. Pathogenetics 2, 4.

Testa, J.R., and Tsichlis, P.N. (2005). AKT signaling in normal and malignant cells. Oncogene 24, 7391-7393.

Teyssou, E., Takeda, T., Lebon, V., Boillee, S., Doukoure, B., Bataillon, G., Sazdovitch, V., Cazeneuve, C., Meininger, V., LeGuern, E., et al. (2013). Mutations in SQSTM1 encoding p62 in amyotrophic lateral sclerosis: genetics and neuropathology. Acta Neuropathol 125, 511-522.

Thomson, J.A., Itskovitz-Eldor, J., Shapiro, S.S., Waknitz, M.A., Swiergiel, J.J., Marshall, V.S., and Jones, J.M. (1998). Embryonic stem cell lines derived from human blastocysts. Science 282, 1145-1147.

Tian, Y., Li, Z., Hu, W., Ren, H., Tian, E., Zhao, Y., Lu, Q., Huang, X., Yang, P., Li, X., et al. (2010). C. elegans screen identifies autophagy genes specific to multicellular organisms. Cell 141, 1042-1055.

Tohyama, S., Hattori, F., Sano, M., Hishiki, T., Nagahata, Y., Matsuura, T., Hashimoto, H., Suzuki, T., Yamashita, H., Satoh, Y., et al. (2013). Distinct metabolic flow enables large-scale purification 
of mouse and human pluripotent stem cell-derived cardiomyocytes. Cell Stem Cell 12, 127-137.

Tsukamoto, S., Kuma, A., Murakami, M., Kishi, C., Yamamoto, A., and Mizushima, N. (2008). Autophagy is essential for preimplantation development of mouse embryos. Science 321, $117-120$.

Tumaneng, K., Russell, R.C., and Guan, K.L. (2012). Organ size control by Hippo and TOR pathways. Curr Biol 22, R368-379.

Urbach, A., Schuldiner, M., and Benvenisty, N. (2004). Modeling for Lesch-Nyhan disease by gene targeting in human embryonic stem cells. Stem cells 22, 635-641.

Vallier, L. (2011). Serum-free and feeder-free culture conditions for human embryonic stem cells. Methods Mol Biol 690, 57-66.

Venugopal, B., Mesires, N.T., Kennedy, J.C., Curcio-Morelli, C., Laplante, J.M., Dice, J.F., and Slaugenhaupt, S.A. (2009). Chaperone-mediated autophagy is defective in mucolipidosis type IV. J Cell Physiol 219, 344-353.

Vergarajauregui, S., Connelly, P.S., Daniels, M.P., and Puertollano, R. (2008). Autophagic dysfunction in mucolipidosis type IV patients. Hum Mol Genet 17, 2723-2737.

Vierbuchen, T., Ostermeier, A., Pang, Z.P., Kokubu, Y., Sudhof, T.C., and Wernig, M. (2010). Direct conversion of fibroblasts to functional neurons by defined factors. Nature 463, 1035-1041.

Wakayama, T., and Yanagimachi, R. (1999). Cloning of male mice from adult tail-tip cells. Nat Genet 22, 127-128.

Wang, S., Xia, P., Rehm, M., and Fan, Z. (2015). Autophagy and cell reprogramming. Cell Mol Life Sci 72, 1699-1713.

Wang, S., Xia, P., Ye, B., Huang, G., Liu, J., and Fan, Z. (2013). Transient activation of autophagy via Sox2-mediated suppression of mTOR is an important early step in reprogramming to pluripotency. Cell Stem Cell 13, 617-625.

Warren, L., Manos, P.D., Ahfeldt, T., Loh, Y.H., Li, H., Lau, F., Ebina, W., Mandal, P.K., Smith, Z.D., Meissner, A., et al. (2010). Highly efficient reprogramming to pluripotency and directed differentiation of human cells with synthetic modified mRNA. Cell Stem Cell 7, 618-630.

Weiwad, W.K., Linke, W.A., and Wussling, M.H. (2000). Sarcomere length-tension relationship of rat cardiac myocytes at lengths greater than optimum. J Mol Cell Cardiol 32, 247-259.

Williams, A., Sarkar, S., Cuddon, P., Ttofi, E.K., Saiki, S., Siddiqi, F.H., Jahreiss, L., Fleming, A., Pask, D., Goldsmith, P., et al. (2008). Novel targets for Huntington's disease in an mTOR-independent autophagy pathway. Nat Chem Biol 4, 295-305.

Wilmut, I., Schnieke, A.E., McWhir, J., Kind, A.J., and Campbell, K.H. (1997). Viable offspring 
derived from fetal and adult mammalian cells. Nature 385, 810-813.

Wu, Y., Li, Y., Zhang, H., Huang, Y., Zhao, P., Tang, Y., Qiu, X., Ying, Y., Li, W., Ni, S., et al. (2015). Autophagy and mTORC1 regulate the stochastic phase of somatic cell reprogramming. Nat Cell Biol 17, 715-725.

Xue, Y., Ouyang, K., Huang, J., Zhou, Y., Ouyang, H., Li, H., Wang, G., Wu, Q., Wei, C., Bi, Y., et al. (2013). Direct conversion of fibroblasts to neurons by reprogramming PTB-regulated microRNA circuits. Cell 152, 82-96.

Yagi, T., Ito, D., Okada, Y., Akamatsu, W., Nihei, Y., Yoshizaki, T., Yamanaka, S., Okano, H., and Suzuki, N. (2011). Modeling familial Alzheimer's disease with induced pluripotent stem cells. Hum Mol Genet 20, 4530-4539.

Yang, Z.J., Chee, C.E., Huang, S., and Sinicrope, F.A. (2011). The role of autophagy in cancer: therapeutic implications. Mol Cancer Ther 10, 1533-1541.

Yu, C., Liu, Y., Ma, T., Liu, K., Xu, S., Zhang, Y., Liu, H., La Russa, M., Xie, M., Ding, S., et al. (2015). Small molecules enhance CRISPR genome editing in pluripotent stem cells. Cell Stem Cell 16, 142-147.

Yu, J.Y., Vodyanik, M.A., Smuga-Otto, K., Antosiewicz-Bourget, J., Frane, J.L., Tian, S., Nie, J., Jonsdottir, G.A., Ruotti, V., Stewart, R., et al. (2007). Induced pluripotent stem cell lines derived from human somatic cells. Science 318, 1917-1920.

Zhang, J. (2015). Teaching the basics of autophagy and mitophagy to redox biologists--mechanisms and experimental approaches. Redox Biol 4, 242-259.

Zhang, J., Wilson, G.F., Soerens, A.G., Koonce, C.H., Yu, J., Palecek, S.P., Thomson, J.A., and Kamp, T.J. (2009). Functional cardiomyocytes derived from human induced pluripotent stem cells. Circ Res 104, e30-41.

Zhang, W.Y., de Almeida, P.E., and Wu, J.C. (2008). Teratoma formation: A tool for monitoring pluripotency in stem cell research. In StemBook (Cambridge (MA)).

Zhao, J., Garcia, G.A., and Goldberg, A.L. (2016). Control of proteasomal proteolysis by mTOR. Nature 529, E1-2.

Zhao, Y.G., Zhao, H., Sun, H., and Zhang, H. (2013). Role of Epg5 in selective neurodegeneration and Vici syndrome. Autophagy 9, 1258-1262. 


\section{Acknowledgements}

I would like to express my deep sense of gratitude to all the people who support me during my doctoral studies over the last three years.

I would like to thank my primary supervisor Prof. Dr. Kaomei Guan. Thank you for providing me with this wonderful opportunity to be a doctoral student and also keen supervision and warm encouragements throughout my project. My appreciation also goes to my secondary supervisor Prof. Dr. Mathias Gautel for his advices, changeling ideas and patient support during my $\mathrm{PhD}$ training.

I would like to thank my committee members Prof. Dr. Dörthe M. Katschinski and Prof. Dr. Peter Rehling for their patience, suggestions and ideas during the annual scientific report.

I am grateful to IRTG1816 and Molecular Medicine Program for the nice organization and giving me an opportunity for international training and further study in Kings College London. Thanks Christina Würtz, Katja El-Armouche, Ulrike Fischer and Erik Meskauskas for dedicated coordination of traveling and administration procedures. I would also thank all the $\mathrm{PhD}$ students to share with me up and down.

Special appreciation goes to all my helpful colleagues from Stem Cell Lab for the friendly and nice atmosphere. Particular thanks to Yvonne Wiegräfe, Johanna Heine, Sarah Henze, Yvonne Hintz and Simin Chen for their helpful support. I would also like to acknowledge Luke Smith, Ay Lin Kho, Eva Masiero and other members in the Mathias Gautel's lab.

My particular thanks go to Poh Loong Soong for his great helpful revision of my thesis.

Last but not least I want to thank my parents and my sister for their support and encouragement. I want to express my deep appreciation to my husband, Xiaopeng Liu, for his love, suggestions and encouragement.

I am happy to meet you in Göttingen! 


\section{Appendix 1: Side project 1}

\section{Patient-specific iPSCs modelling TTN mutation caused cardiomyopathy}

\section{Introduction}

Titin (TTN) is a giant protein with molecular weight $\sim 4000 \mathrm{kDa}$ found in striated muscles. It is encoded by the TTN gene that consists of 364 exons. The structure of the TTN protein is composed of protein domains with highly repetitive sequences, which include immunoglobulin-like domains ( $\operatorname{IgG}, \mathrm{n}=152)$, fibronectin 3 (Fn3, $\mathrm{n}=132$ ), regulator of chromosome condensation 1 ( $\mathrm{RCC} 1, \mathrm{n}=17)$, Kelch $(\mathrm{n}=19)$, tetratricopeptide repeat (TPR, $\mathrm{n}=14)$, solenoid WD domains $(\mathrm{n}=15)$ and PEVK domains (proline, glutamate, valine and lysine, $n=60$ ). In addition, TTN protein contains a titin kinase (TK) domain (Chauveau et al., 2014).

There are three main titin isoforms: N2A, N2B and N2BA, as well as three rare isoforms: NOVEX1, NOVEX2 and NOVEX3 (Bang et al., 2001; Furst et al., 1988; Hackman et al., 2002; Linke and Hamdani, 2014; Miller et al., 2003; Vikhlyantsev and Podlubnaya, 2012). The TTN gene could theoretically generate over 1 million splice variants with a wide range of protein size. However, the number of TTN protein variants thus far demonstrated experimentally is lower than expected. NOVEX3 ( 616 kDa) is the smallest isoform of TTN and expressed in all striated muscles; N2A ( 3680 kDa) has been identified as the main TTN isoform in the skeletal muscle; in cardiomyocytes, there are two main cardiac isoforms N2B ( 2960 kDa) and N2BA $(\sim 3780 \mathrm{kDa})$ and additional isoforms NOVEX1 ( 2980 kDa) and NOVEX2 ( 2980 kDa) (Bang et al., 2001; Freiburg et al., 2000).

TTN is the only single molecular element spanning from the $\mathrm{Z}$ disk to the $\mathrm{M}$ line in the sarcomere, which is important for the sarcomere assembly, stabilization and muscle elasticity. This has been demonstrated in a variety of experiments, for example, zebrafish mutants of cardiac specific N2B demonstrated poor contractility from the first beat, 
nascent myofibril formation and abnormal sarcomere structure (Xu et al., 2002). Specifically knocking-out only the N2B exon 49 in mice showed that N2B region is important for the elastic functions of the heart (Radke et al., 2007). Deletion of the TK domain from cardiac TTN in mice leads to the absence of homozygous offspring (Gotthardt et al., 2003). Additionally, next generation sequencing revealed that mutations in TTN (mostly heterozygous) were observed in dilated cardiomyopathy (DCM), hypertrophic cardiomyopathy (HCM), arrhythmogenic right ventricular cardiomyopathy (ARVC) and restrictive cardiomyopathy (RCM). Furthermore, due to the difficulty for acquiring human heart tissue and size of titin protein, little is known about the precise molecular and pathophysiological mechanisms related with different TTN mutations. Thus, induced pluripotent stem cells (iPSCs) provide a good platform to generate unlimited cardiomyocytes and might be suitable to study the cardiac phenotype and the underlying molecular mechanism of cardiomyopathy caused by TTN mutations. Cardiomyocytes derived from DCM patient-specific iPSCs with TTN mutations showed sarcomere insufficiency, impaired responses to mechanical and beta-adrenergic stress, and attenuated growth factor and cell signaling activation (Hinson et al., 2015).

In this study, iPSCs were generated from a DCM patient with compound heterozygous TTN mutations (c.54710T $>\mathrm{C}$ and c.106359G $>\mathrm{A}$ ) and differentiated into functional cardiomyocytes. The phenotype of patient-specific cardiomyocytes and the expression of TTN protein was investigated.

\section{Materials and methods}

\subsection{TTN-iPSC generation}

Patient-specific iPSCs were reprogrammed from the fibroblasts, which were derived from a DCM patient with compound heterozygous mutations (c.54710 T>C and c.106359 G>A) in TTN. Fibroblasts at passage 8 were transduced with Sendai virus reprogramming kit (Thermo Fisher Scientific) at MOI 2.5-2.5-1.5. The independent single iPSC colonies were picked 30 days post transduction and extended to generate stable iPSC lines. Among the cell lines, isTTN1.1, isTTN1.3 and isTTN1.4 were characterized for the pluripotency 
and were used for subsequent experiments and studies.

\subsection{Titin protein detection}

Low percentage gel (2\%) consisting of $2.5 \mathrm{ml}$ buffer (1.5 M Tris ( $\mathrm{pH} 8.8$ ) with $0.4 \%$ SDS), $2.95 \mathrm{ml} \mathrm{H} 2 \mathrm{O}, 0.7 \mathrm{ml} \mathrm{30 \%}$ acrylamide, $100 \mu \mathrm{l}$ APS, $5.75 \mu \mathrm{l}$ TEMED and $3.39 \mathrm{ml}$ $1.5 \%$ agar was used to detect the TTN protein. The gels were subjected to electrophoresis for 12-16 $\mathrm{h}$ at low voltage ( $20 \mathrm{~V})$ and further used in coomassie staining and Western blot. TTN protein was detected with primary antibodies against the titin domain M8 and Z1Z2 (antibodies were produced in Mathias Gautel's lab).

\subsection{RT-PCR}

Total RNA was isolated using the SV total RNA isolation system (Promega) according to the manufacturer's instructions. 200 ng complementary DNA (cDNA) was synthesized from isolated RNA with the reverse transcriptase (Invitrogen). The specific DNA products of titin were amplified with polymerase chain reaction (PCR). The primers for detecting two mutation sites (c.54710T $>\mathrm{C}$ and c.106359G $>$ A) were: for: GCTGGACTCCTCCTTTGGAC, rev: ATATGGGATCTCCTGCAACCTCT; and for: CTGAACCAAAAGCTCCTGAACCAATTTC, rev: TCCTTGTGTAATGGCCTGTAGA ATGCAA, respectively.

\subsection{Immunofluorescence staining}

Cardiomyocytes fixed by $4 \%$ PFA were blocked with $1 \%$ BSA, and following permeabilization by $0.1 \%$ Triton X100 (Sigma), they were stained with primary antibodies against $\alpha$-ACTININ, cTNT, MYOMESIN (M line binding protein) and TTN. The antibodies for TTN include M8, M8A5, T12 and Z1Z2.

Other methods used in this study were referred in the first chapter of this thesis.

\section{Results}

To investigate the cardiac phenotype at cellular level, patient-specific iPSCs (TTN-iPSCs) were successfully generated through overexpression of the transcription factors OCT4, 
SOX2, KLF4 and c-MYC using the Sendai virus system. After cellular reprogramming, compound heterozygous mutations (c.54710T $>\mathrm{C}$ and c.106359G $>\mathrm{A}$ ) in the TTN gene were verified in TTN-iPSCs (Figure 1D). Furthermore, analysis of pluripotency showed that these TTN-iPSCs were pluripotent, including positive alkaline phosphatase staining, expression of pluripotent-related markers OCT4, SOX2, NANOG, LIN28, SSEA4 and TRA1-60, normal karyotype (23+XY), capacity of differentiation into derivatives of three germ layers in vitro and in vivo (Figure 1A-C, E, F).

To study the effect of $T T N$ mutations on sarcomeric structure, these generated pluripotent TTN-iPSCs were directly differentiated into beating cardiomyocytes in vitro. $90 \%$ pure cardiomyocytes were obtained using our cardiac differentiation protocol. Cardiomyocytes derived from health control iPSCs (Ctr-iPSC-CMs) and TTN-iPSCs (TTN-iPSC-CMs) highly expressed cardiac-related markers such as TNNT2, MYL2, MYH7, NKX2.5 on gene transcription level demonstrated by the RT-PCR analysis and $\alpha$-ACTININ and cTNT on protein level demonstrated by immunofluorescence staining (Figure 2A-C).

Next, to identify the cardiac phenotype caused by the TTN mutation, Ctr- and TTN-iPSC-CMs were immunostained with primary antibodies against the M line (M8 and M8A5) and the Z disk (T12 and Z1Z2) of TTN (Figure 3A and C). M8A5 was specifically designed at the mutation site of TTN protein (c.106359 G>A, Trp to stop codon). The data demonstrated that TTN protein was not detectable in TTN-iPSC-CMs using the M8A5 antibody in contrast to the regular distribution of TTN with striated sarcomere structures in Ctr-iPSC-CMs (Figure 3C). Additionally, the primary antibody targeting the M line of TTN protein (M8) was also unable to detect clearly the TTN protein in TTN-iPSC-CMs (Figure 3A). Sarcomeric structures of TTN protein were only observed when the cells were incubated with specific antibody targeting the Z disk (T12 and Z1Z2) in both TTN-iPSC-CMs and Ctr-iPSC-CMs (Figure 3A and C). The results from the Western blot further corroborated the immunofluorescence staining data, demonstrating that TTN protein was not detectable with the M8 antibody, but visible with Z1Z2 in TTN-iPSC-CMs. Three isoforms were detected by the Western blot: the very 
long embryonic/fetal N2BA isoform, the adult N2B isoform and the truncated NOVEX3 isoform (Figure 3B and D).

Mutation in the TTN gene is heterozygous, but the proteins were not detectable with the antibody specific for the M line of TTN. Hence, we hypothesize that the other mutation at c.54710 $\mathrm{T}>\mathrm{C}$ might lead to an additional truncated protein, which is degraded through autophagy or ubiquitin proteasome system (UPS) processing. In order to determine and detect the other truncated protein, cardiomyocytes were treated with autophagy inhibitor bafilomycin (100 nM, $24 \mathrm{~h})$ and UPS inhibitor IMG132 (50 $\mu \mathrm{M}, 24 \mathrm{~h})$. The results of immunofluorescence staining showed that TTN protein were still not detectable using the M8 antibody (Figure 4A and $\mathbf{B}$ ) whereas other $\mathrm{M}$ line binding protein such as MYOMESIN was normally expressed in TTN-iPSC-CMs (Figure 4A). The degraded proteins were visible after the bafilomycin treatment on Western blot using the antibody Z1Z2 (Figure 4D, white arrow). 
A

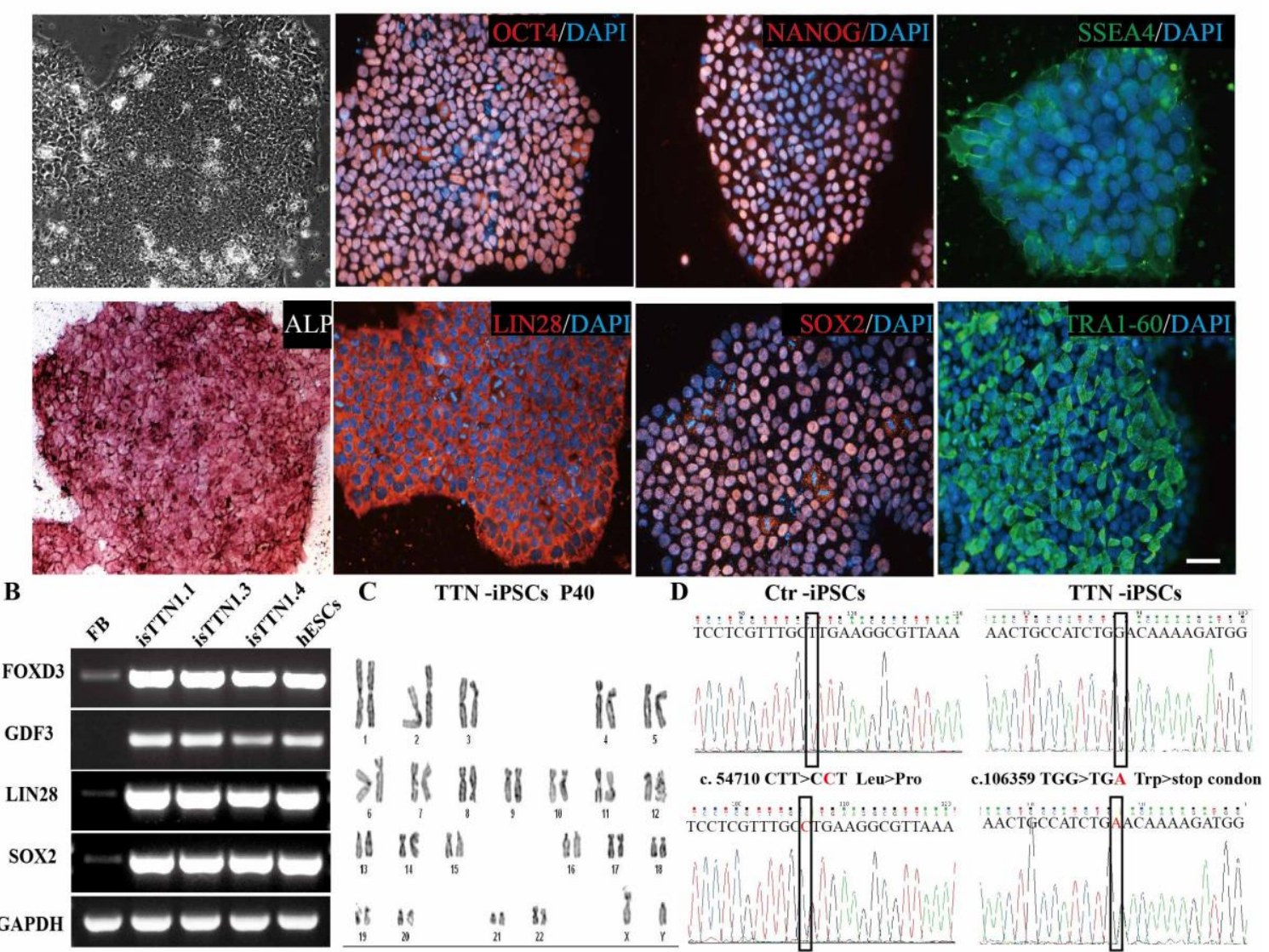

$\mathbf{E}$
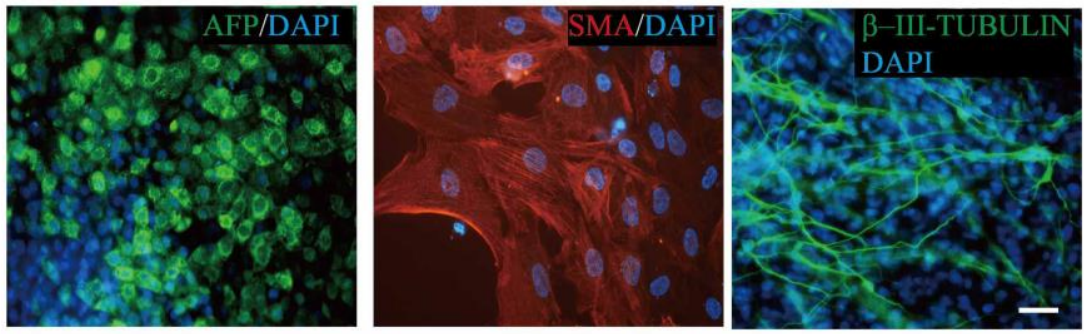

$\mathbf{F}$
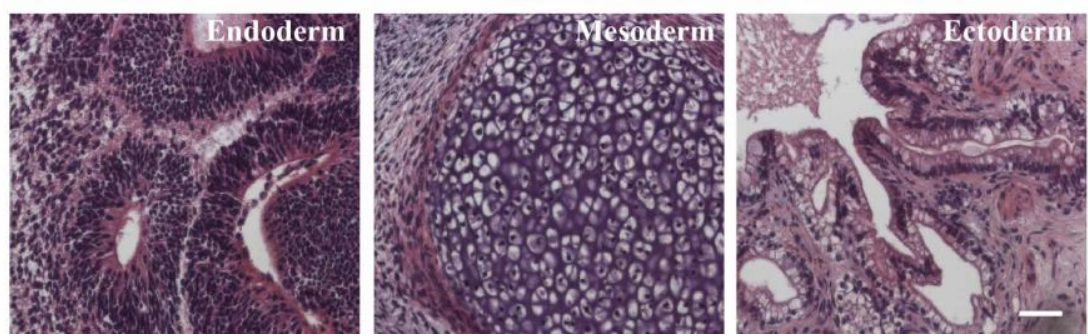

Figure 1: Proof of pluripotency in TTN-iPSCs reprogrammed from TTN fibroblasts. (A) Representative TTN-iPSCs were alkaline phosphatase positive and expressed pluripotency markers OCT4, SOX2, NANOG, LIN28, SSEA4 and TRA-1-60. (B) All three TTN-iPSC lines showed high expression of pluripotent-related genes including FOXD3, GDF3, LIN28 and SOX2. hESCs: human embryonic stem cells; FB, fibroblasts isolated from the TTN patient; isTTN1.1, isTTN1.3 and isTTN1.4, three iPSC lines derived from the TTN patient. (C) Karyotype analysis showed the normal karyotypes of TTN-iPSCs at passage 40. (D) Compound heterozygous TTN mutations c.54710T $>\mathrm{C}$ and c.106359G $>$ A were verified in derived iPSCs. (E) TTN-iPSCs spontaneously differentiated into cells of three different embryonic germ layers in vitro. The endoderm representative marker is alpha fetoprotein (AFP); the mesoderm representative marker 
is smooth muscle actin (SMA) and ectoderm specific marker $\beta$-III-TUBULIN. The nuclei were stained with DAPI. (F) Histological staining of teratoma from TTN-iPSCs showed in vivo generation of derivatives of three embryonic germ layers including intestinal (endoderm), cartilage (mesoderm) and neural tissues (ectoderm). Scale bar: $50 \mu \mathrm{m}$.

A

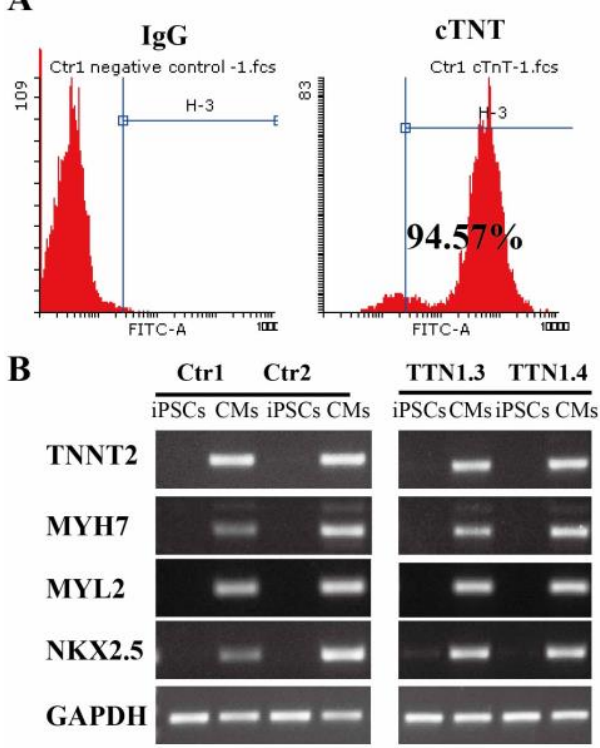

C
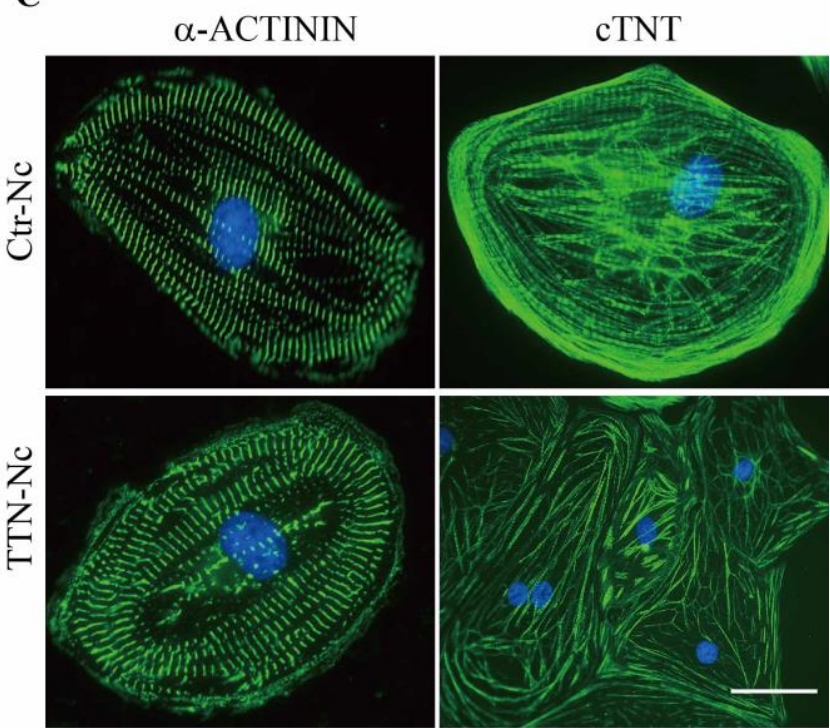

Figure 2. Cardiac differentiation of Ctr- and TTN-iPSCs. (A) Both Ctr- and TTN-iPSCs were differentiated into functional cardiomyocytes with high efficiency. (B) Ctr- and TTN-iPSC-CMs expressed the cardiac-related genes including TNNT2, MYH7, MYL2 and NKX2.5. (C) Ctr- and TTN-iPSC-CMs showed expression of cTNT and Z-disk binding protein $\alpha$-ACTININ. Scale bar: $50 \mu \mathrm{m}$. 
A
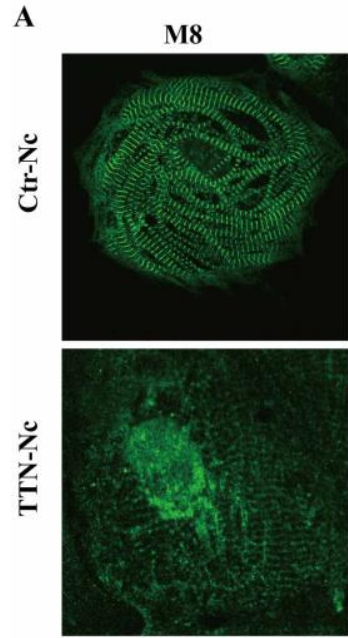

C

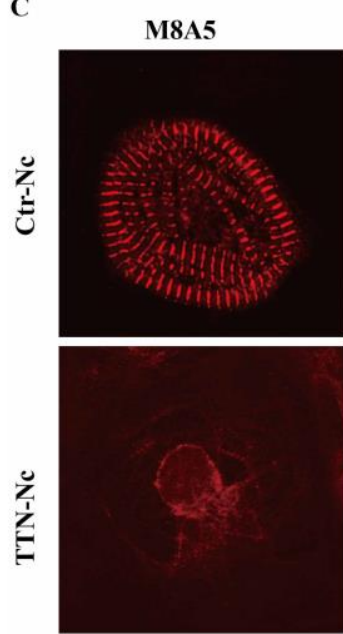

T12
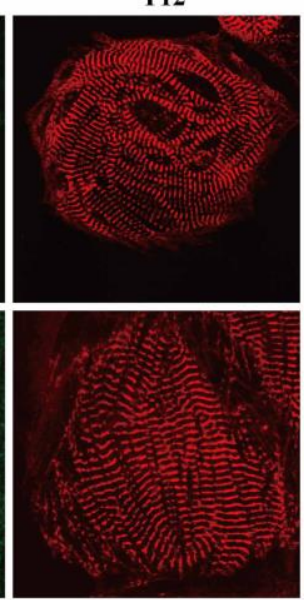

Z1Z2

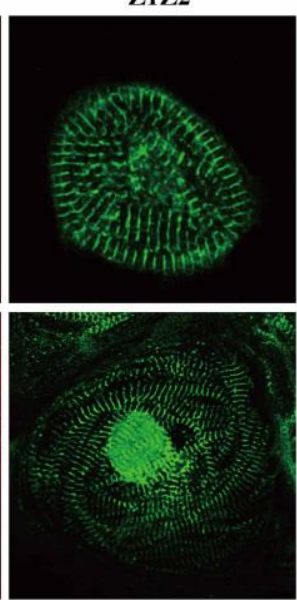

Merge
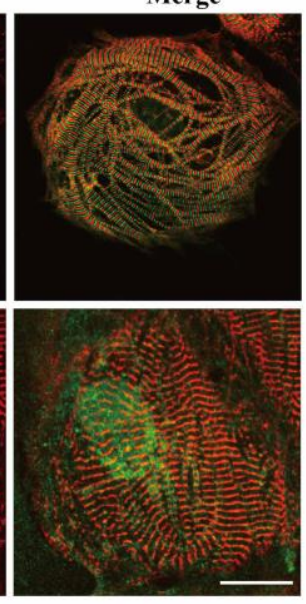

Merge

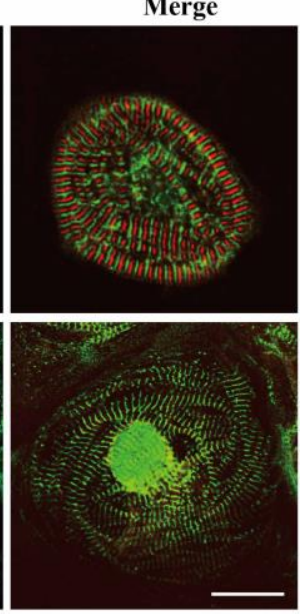

B

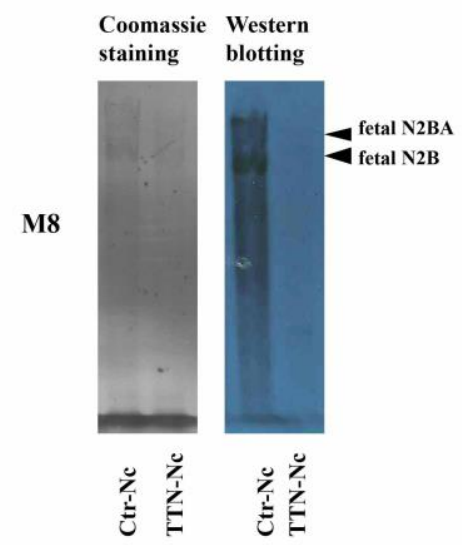

D

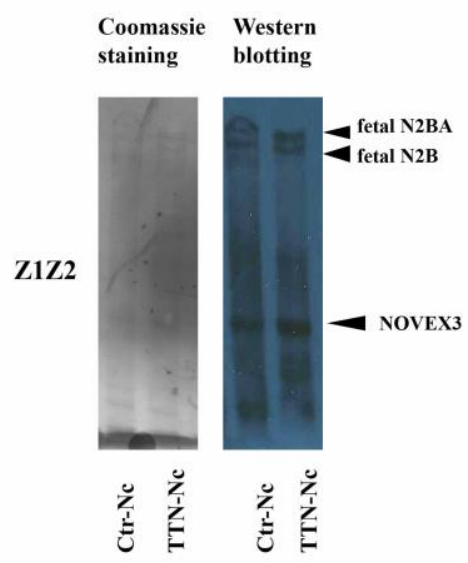

Figure 3. The M line of TTN was not visible in TTN-iPSC-CMs. (A) TTN-iPSCs-CMs showed abnormal expression of the $\mathrm{M}$ line of TTN protein using the M8 antibody. (B) TTN protein cannot be detected through Western blot analysis with primary antibody against M8 in TTN-iPSC-CMs. (C) The M line of TTN protein was not visible with specific antibody (M8A5) against the mutation site (c.106359G>A) in TTN-iPSCs-CMs. (D) Two fetal cardiac TTN isoforms $\mathrm{N} 2 \mathrm{~B}$ and $\mathrm{N} 2 \mathrm{BA}$ as well as NOVEX3 were detected using the antibody Z1Z2 in TTN-iPSC-CMs. Scale bar: $50 \mu \mathrm{m}$. 


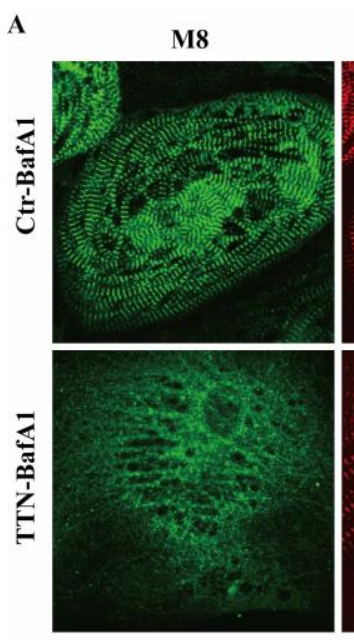

B
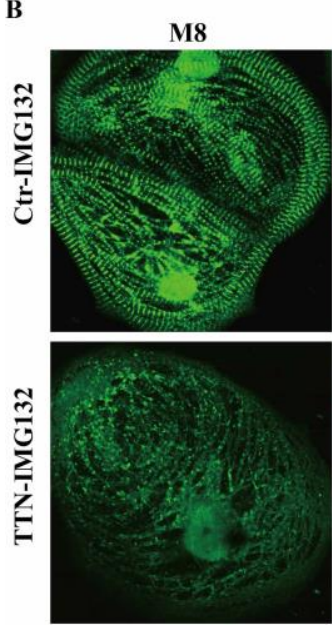

MYOMESIN

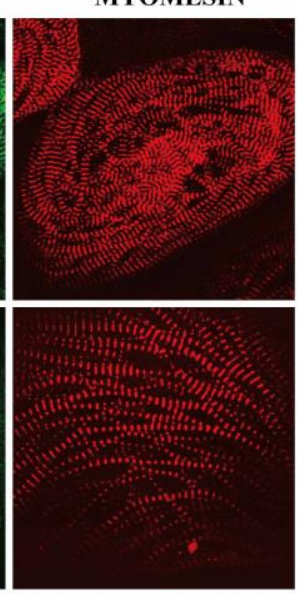

T12
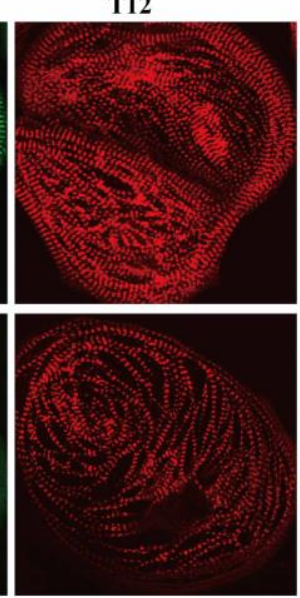
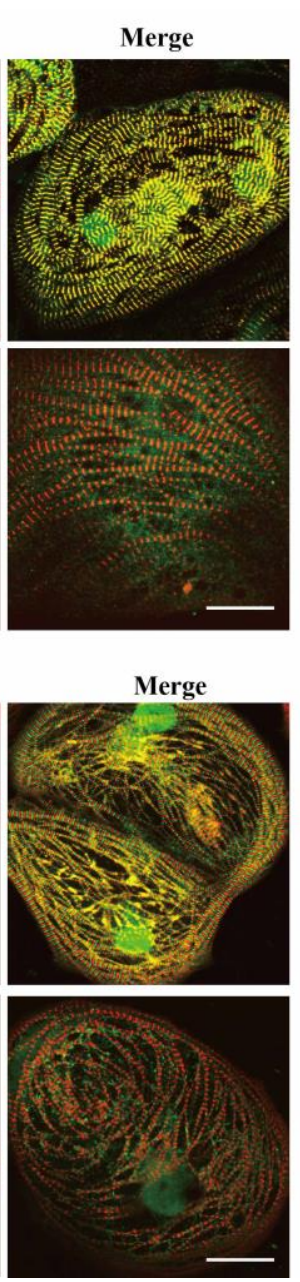

C

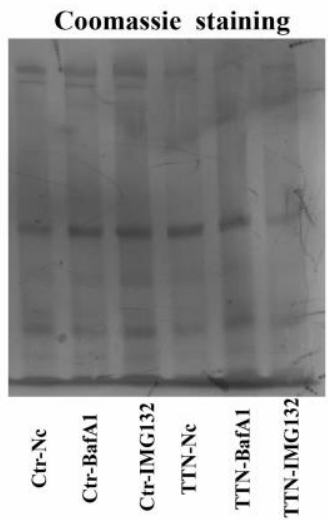

D

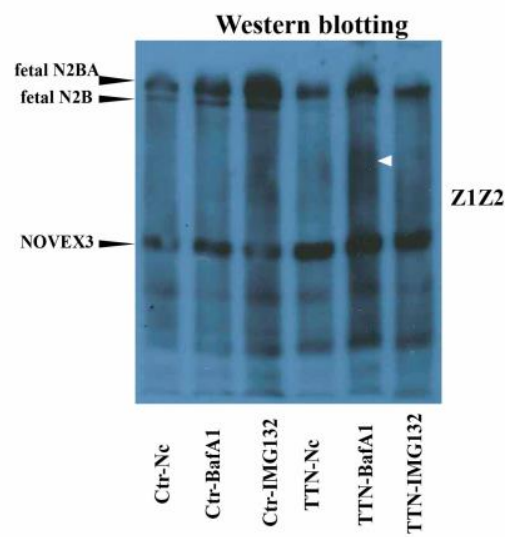

Figure 4: Detection of TTN protein after bafilomycin and IMG132 treatment. (A, B) TTN protein were not be detected as sarcomeric structures using the M8 antibody in TTN-iPSC-CMs even after blocking the protein degradation pathway by bafilomycin (BafA1) and IMG132, respectively. The sarcomere structure was stained with the antibody against the $\mathrm{Z}$ disk of TTN (T12) and MYOMESIN. (C, D) Western blot analysis showed the degraded protein is visible after bafilomycin treatment in TTN-iPSC-CMs (white arrow). Scale bar: $50 \mu \mathrm{m}$.

\section{Discussion}

Next generation sequencing technologies revealed that $T T N$ mutations were associated with cardiomyopathy. However, the underlying molecular mechanisms have not been clearly demonstrated. The current molecular study of TTN mutations and cardiomyopathy is limited due to the technical difficulty for cloning the full-length TTN gene, purification of this giant protein and obtaining native heart muscle. The mutation sites in the TTN gene were diverse and located in the $\mathrm{Z}$ disk, I band, A band and $\mathrm{M}$ line of the sarcomere. The TTN mutation investigated in this study is compound heterozygous, with a missense mutation (c.54710T >C, Leu>Pro) in the A band and a truncate mutation (c.106359G >A, 
Trp to stop codon) in the $\mathrm{M}$ line. TTN protein plays a critical role at the $\mathrm{M}$ line to maintain structural integrity of sarcomere and myofibrillar signaling. The conditional knockout of TK domain in the M line in mice showed that homozygous deletion of the domain led to embryonic lethality whereas heterozygous deletion resulted in sarcomere structure disassembly (Gotthardt et al., 2003; Peng et al., 2005; Weinert et al., 2006). In this study, the mutation c.106359G > A (Trp to stop codon) in TTN leads to a truncated TTN protein at the $\mathrm{M}$ line, which is not detectable using the $\mathrm{M}$ line-specific antibodies (M8 and M8A5) as expected. However, as the mutation c.106359G>A (Trp to stop codon) is heterozygous, we do expect to detect TTN protein using the M line-specific antibodies in TTN-iPSC-CMs. Surprisingly, TTN protein was completely not detectable with specific antibodies M8 and M8A5 in TTN-iPSC-CMs. These data suggest that the other mutation at c.54710T $>\mathrm{C}$ in the other allele of TTN might lead to TTN protein instability and degradation. The unstable or truncated proteins might be degraded through autophagy or ubiquitin proteasome system (UPS) processing. Two fetal isoforms of TTN (N2B and N2BA) were detected with the antibody Z1Z2, but Western blot was unable to distinguish the truncated TTN from the normal TTN, due to the resulting similar protein size. In this study, a slightly enhanced degradation of TTN was observed only after blocking protein degradation pathways. Further studies need to investigate how the mutation c.54710T $>\mathrm{C}$ affects the TTN protein stability and degradation. Taken together, this study demonstrated that TTN-iPSCs could be used to elucidate the physiological role of different TTN domains and allow better understanding of the role of TTN in cardiomyopathy. 


\section{References}

Bang, M.L., Centner, T., Fornoff, F., Geach, A.J., Gotthardt, M., McNabb, M., Witt, C.C., Labeit, D., Gregorio, C.C., Granzier, H., et al. (2001). The complete gene sequence of titin, expression of an unusual approximately $700-\mathrm{kDa}$ titin isoform, and its interaction with obscurin identify a novel Z-line to I-band linking system. Circ Res 89, 1065-1072.

Freiburg, A., Trombitas, K., Hell, W., Cazorla, O., Fougerousse, F., Centner, T., Kolmerer, B., Witt, C., Beckmann, J.S., Gregorio, C.C., et al. (2000). Series of exon-skipping events in the elastic spring region of titin as the structural basis for myofibrillar elastic diversity. Circ Res 86 , 1114-1121.

Furst, D.O., Osborn, M., Nave, R., and Weber, K. (1988). The organization of titin filaments in the half-sarcomere revealed by monoclonal antibodies in immunoelectron microscopy: a map of ten nonrepetitive epitopes starting at the $\mathrm{Z}$ line extends close to the $\mathrm{M}$ line. J Cell Biol 106, 1563-1572.

Gotthardt, M., Hammer, R.E., Hubner, N., Monti, J., Witt, C.C., McNabb, M., Richardson, J.A., Granzier, H., Labeit, S., and Herz, J. (2003). Conditional expression of mutant M-line titins results in cardiomyopathy with altered sarcomere structure. J Biol Chem 278, 6059-6065.

Hackman, P., Vihola, A., Haravuori, H., Marchand, S., Sarparanta, J., De Seze, J., Labeit, S., Witt, C., Peltonen, L., Richard, I., et al. (2002). Tibial muscular dystrophy is a titinopathy caused by mutations in TTN, the gene encoding the giant skeletal-muscle protein titin. Am J Hum Genet 71, 492-500.

Hinson, J.T., Chopra, A., Nafissi, N., Polacheck, W.J., Benson, C.C., Swist, S., Gorham, J., Yang, L., Schafer, S., Sheng, C.C., et al. (2015). HEART DISEASE. Titin mutations in iPS cells define sarcomere insufficiency as a cause of dilated cardiomyopathy. Science 349, 982-986.

Linke, W.A., and Hamdani, N. (2014). Gigantic business: titin properties and function through thick and thin. Circ Res 114, 1052-1068.

Miller, G., Musa, H., Gautel, M., and Peckham, M. (2003). A targeted deletion of the C-terminal end of titin, including the titin kinase domain, impairs myofibrillogenesis. J Cell Sci 116, 4811-4819.

Peng, J., Raddatz, K., Labeit, S., Granzier, H., and Gotthardt, M. (2005). Muscle atrophy in titin M-line deficient mice. J Muscle Res Cell Motil 26, 381-388.

Radke, M.H., Peng, J., Wu, Y., McNabb, M., Nelson, O.L., Granzier, H., and Gotthardt, M. (2007). Targeted deletion of titin N2B region leads to diastolic dysfunction and cardiac atrophy. Proc Natl Acad Sci U S A 104, 3444-3449.

Vikhlyantsev, I.M., and Podlubnaya, Z.A. (2012). New titin (connectin) isoforms and their functional role in striated muscles of mammals: facts and suppositions. Biochemistry (Mosc) 77, $1515-1535$. 
Weinert, S., Bergmann, N., Luo, X., Erdmann, B., and Gotthardt, M. (2006). M line-deficient titin causes cardiac lethality through impaired maturation of the sarcomere. J Cell Biol 173, 559-570.

Xu, X., Meiler, S.E., Zhong, T.P., Mohideen, M., Crossley, D.A., Burggren, W.W., and Fishman, M.C. (2002). Cardiomyopathy in zebrafish due to mutation in an alternatively spliced exon of titin. Nat Genet 30, 205-209. 


\section{Appendix 2: Side project 2}

\section{Direct differentiation of skeletal muscle cells from human induced pluripotent stem} cells

\section{Introduction}

$\mathrm{X}$-linked myotubular myopathy (XLMTM) is an inherited neuromuscular disorder, which predominantly affects females and is characterized by muscle weakness present at birth, hypotonia, feeding difficulties and inability to maintain spontaneous respiration (Wallgren-Pettersson and Thomas, 1994). XLMTM is caused by mutations in the MTM1 gene (in Xq28), encoding the myotubularin protein which functions as a phosphatase acting specifically on phosphatidylinositol 3-phosphate (PI(3)P) and phosphatidylinositol 3,5-bisphosphate $(\mathrm{PI}(3,5) \mathrm{P} 2)$. Phosphoinositides (PIs) play an important role in lipid metabolisms, cell signaling and membrane trafficking. $\mathrm{PI}(3) \mathrm{P}$ and $\mathrm{PI}(3,5) \mathrm{P} 2$ directly participate in the endosomal-lysosome pathway (Heckmatt et al., 1985; Robinson and Dixon, 2006). Recently, defective PI conversion at endosomes was shown in the fibroblasts derived from XLMTM patients (Ketel et al., 2016). However, the studies on disease phenotype of skeletal muscle and the underlying molecular mechanisms in XLMTM patients were impeded due to the limited availability of the muscle biopsy. Human iPSCs are self-renewal and can differentiate in vitro into skeletal muscle cells, potentially providing unlimited supply of these tissues. Generating patient-specific skeletal muscle cells to model XLMTM in vitro provide new insights into our understanding of key signaling pathways and disease mechanisms involved in XLMTM.

Current studies of myogenic differentiations from iPSCs rely on cell sorting or ectopic expression of myogenic regulators or small molecules. In a previously published study, hESCs were first co-cultured with stromal cells OP9 to induce mesenchymal precursor, which were sorted with CD73 and further co-cultured with $\mathrm{C} 2 \mathrm{C} 12$ to lead the formation of myotubes (Barberi et al., 2005). Furthermore, the myogenic differentiation of hESCs and iPSCs was induced by formation of embryoid bodies (Awaya et al., 2012; Zheng et al., 2006). However, myogenic differentiation based on the EB protocol was inefficient. 
To overcome these limitations, iPSCs were ectopically transduced with MYOD and PAX7 using lentivirus or adenovirus to efficiently induce the generation of skeletal muscle cells (Darabi et al., 2012; Tanaka et al., 2013; Tedesco et al., 2012). In addition, a monolayer culture for myogenic differentiation, the non-transgenic method was performed by the sequential treatment with the GSK3 $\beta$ inhibitor CHIR99021 and bFGF in serum-free medium (Borchin et al., 2013; Shelton et al., 2014). Previous study used GSK3 $\beta$ inhibitor BIO, cAMP activator forskolin and bFGF to induce iPSCs to form EBs and further specifically differentiate into skeletal muscle cells (Xu et al., 2013). Furthermore, the low concentration $\left(10^{-8} \mathrm{M}\right)$ of retinol acids (RA) was shown to induce skeletal muscle cell generation from embryonic carcinoma cells (Edwards and McBurney, 1983).

Based on these previous studies, a directed differentiation protocol was established in this study to induce iPSCs into skeletal muscles with chemical molecules with high efficiency. To elucidate the muscle phenotype of XLMTM, iPSCs were generated from two XLMTM patients with MTM1 mutations (c.141_144 del AGAA and c.231+1G>A, respecitively). These iPSCs were further analyzed regarding their pluripotency.

\section{Materials and methods}

\subsection{RT-PCR}

RNA was isolated and transcribed to cDNA according to the manufacturer's instructions. The target genes were amplified with PCR, including PAX3, PAX7, MYOD, MYOGENIN and $M Y H 7$. The primers were:

PAX3: for: TACAGGTCTGGTTTA GCAAC, rev: GATCTGACACAGCTTGTGGA; PAX7: for: ACCCCTGCCTAA CCACATC, rev: GCGGCAAAGAATCTTGGAGAC; MYOD: for: TGCCACAACGGACGACTTC, rev: TCTTGCGCTTGCACGCCTT; MYOGENIN: for: AGATGTGTCTGTGGCCTTC, rev: AGCTGGCTTCCTAGCATCAG; MYH7, for: TTCATTGGGGTCTTGGACATTG, rev: GAACGTCCACTCAATGCCTTC. 


\subsection{Immunofluorescence staining}

Directly differentiated skeletal muscle cells were fixed with $4 \%$ PFA and blocked with $1 \%$ BSA. Following permeabilization with $0.1 \%$ Triton X100, cells were stained with primary antibodies against $\alpha$-ACTININ (Sigma), Nebulin (Sigma), Desmin (Abcam) and MHC protein (R\&D).

\section{3 iPSC generation}

Human fibroblasts derived from XLMTM patients with MTM1 mutations (c.141_144 del AGAA and c.231+1G>A) were transduced with STEMCCA virus containing the transcription factors OCT4, SOX2, KLF4 and c-MYC. iPSC colonies were picked 35-40 days post transduction and cultivated as stable cell lines, iXLMTM1.1, 1.2, 1.3 and iXLMTM2.1, 2.2 and 2.4.

Other methods in details are referred to the first chapter of this thesis.

\section{Results}

In order to obtain skeletal muscle cells including progenitor myoblasts, nascent myotubes and beating myotubes, hiPSCs were seeded and cultivated into colonies with diameter of 300-400 $\mu \mathrm{m}$ in Essential 8 medium (Invitrogen) with pro-survival factors (Merck). The differentiation was initiated with supplementation of $4 \mu \mathrm{M}$ GSK3 $\beta$ inhibitor CHIR99021 (Merck) for 4 days in DMEM/F-12 (Life technology). Thereafter, medium was changed to DMEMF/12 supplemented with $10 \mathrm{ng} / \mathrm{ml} \mathrm{bFGF} \mathrm{(Peprotech),} 20 \mu \mathrm{g} / \mathrm{ml}$ forskolin (Sigma) and $10 \mathrm{ng} / \mathrm{ml} \mathrm{RA} \mathrm{(Sigma)} \mathrm{for} \mathrm{another} 14$ days, which induce skeletal muscle progenitor's generation. Skeletal muscle progenitors proliferated in the skeletal muscle growth medium (Promocell) for another 10-14 days. In addition, to enable myocyte fusion, skeletal muscle differentiation medium (Promocell) was used until day 45 to day 60 (Figure 1A).

Due to the modified differentiation protocol, the myocytes, nascent myotubes, fusing myotubes and beating myotubes were observed (Figure 1B). Furthermore, confirmation with fluorescence staining was performed and revealed that myocytes with single nuclei 
and fused myotubes with multiple nuclei were $\alpha$-ACTININ positive (Figure 2). Other striated muscle markers DESMIN and MHC were also highly expressed in these differentiated myocytes and myotubes (Figure 3B, C). The staining of specific marker for skeletal muscle cells demonstrated that they were NEBULIN-positive, indicating that these differentiated cells were skeletal muscle cells or their progenitors (Figure 3A). On transcript level, RT-PCR results showed high expression of skeletal muscle-specific markers MYOD, MYOGENIN and MYH7 as well as their progenitors-specific markers $P A X 3$ and $P A X 7$ (Figure 3D).

The XLMTM patient-specific iPSCs (XLMTM-iPSCs) were reprogrammed from the fibroblasts derived from the biopsy of the donors who carry the MTM1 mutation (c.141_144 del AGAA and c.231+1G>A). Through characterization of pluripotency, the results illustrated that patient-specific iPSCs were pluripotent including positive alkaline phosphatase staining, high expression of pluripotency markers OC4, SOX2, NANOG, LIN28, SSEA4 and TRA1-60 and capacity of differentiation into derivatives of three germ layers in vitro and in vivo (Figure 4 and 5).

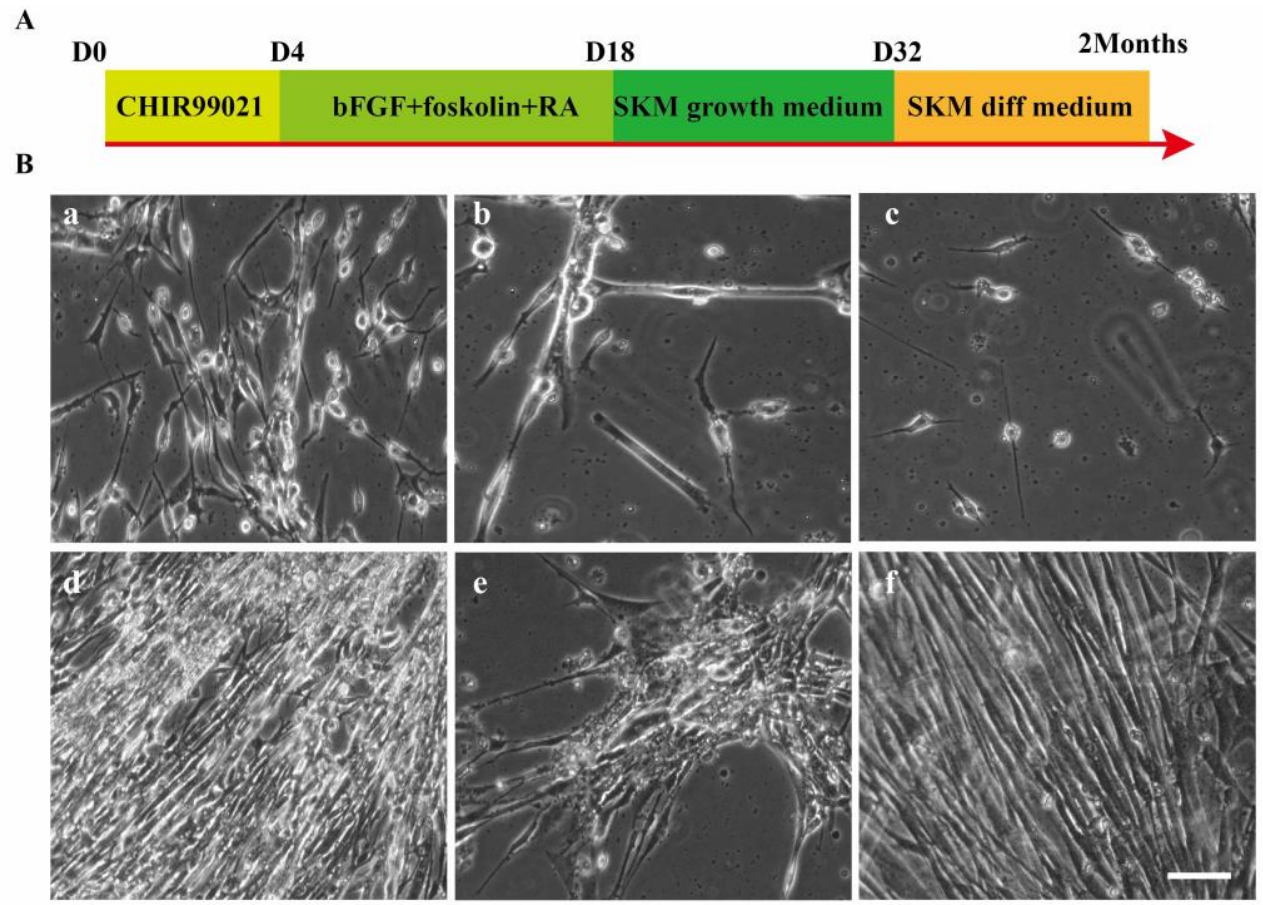

Figure 1: Skeletal muscle differentiation from Ctr-iPSCs. Myotubes were differentiated using the protocol shown in $\mathbf{A}$ and bright field images were taken to show nascent myotubes at day 40 (a, b), myoblasts and myocytes at day 30 (c) and beating myotubes at day 60 (d-f) (B). Scale bar: $50 \mu \mathrm{m}$. 

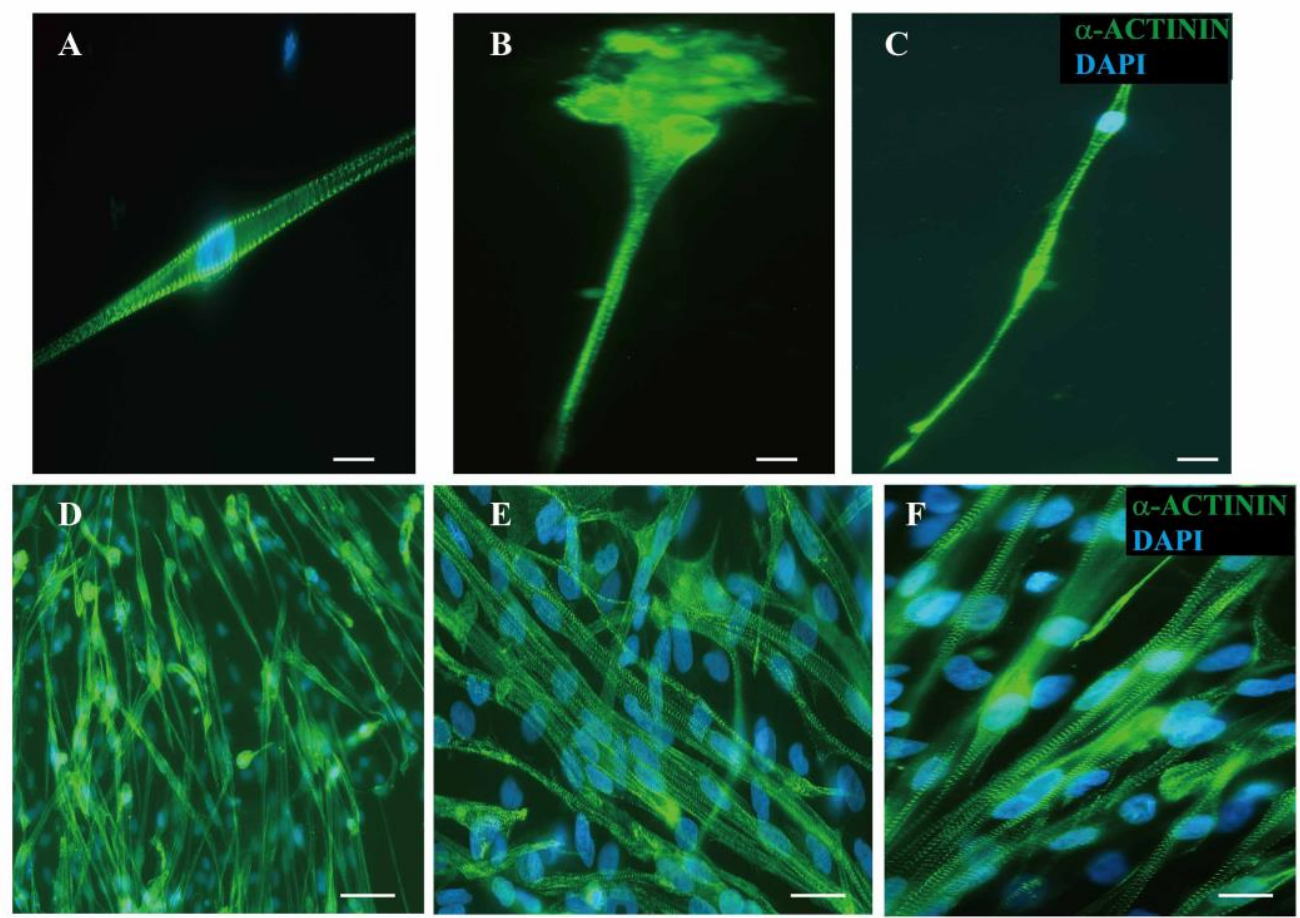

Figure 2. Immunofluorescence staining of direct differentiated skeletal muscle cells. Sarcomere structure was shown in myocytes (A-C) at day 40 and myotubes at day 60 (D-E) stained with antibodies against $\alpha$-ACTININ. Scale bar: $50 \mu \mathrm{m}$.
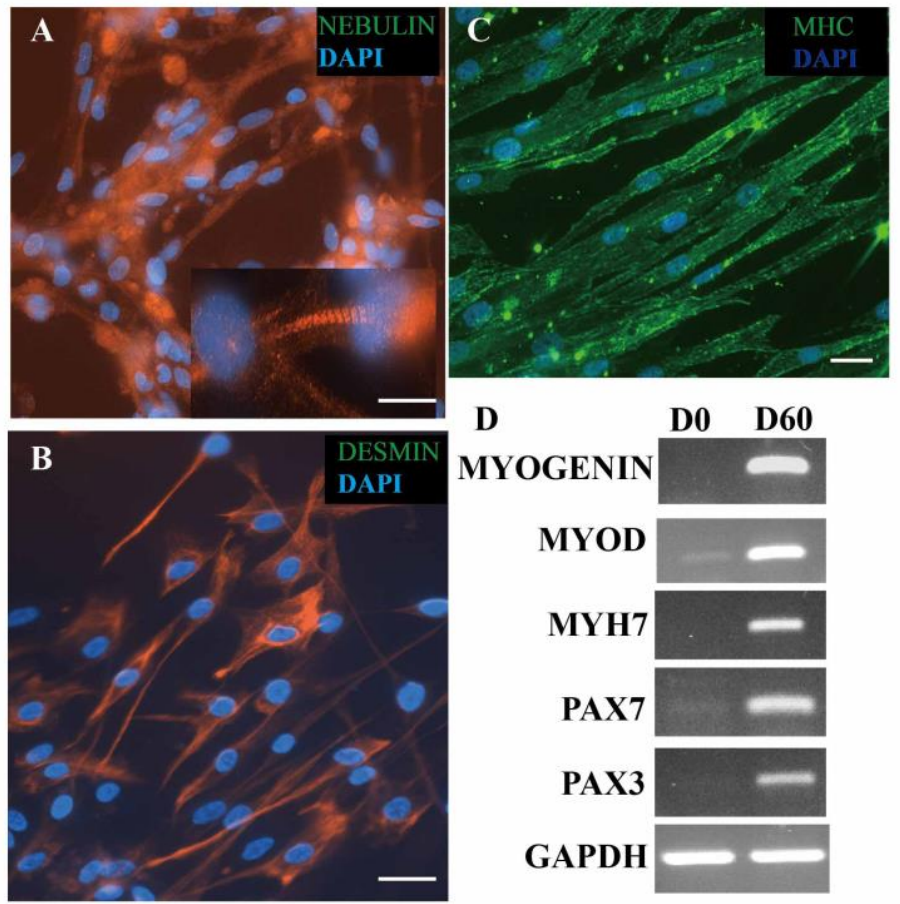

Figure 3. Expression of skeletal muscle-related markers on protein and mRNA level. Sarcomere structure of myotubes at day 60 was shown stained with antibodies against other striated muscle markers including NEBULIN (A), DESMIN (B) and MHC (C). The generated myotubes highly expressed myogenic markers including PAX3, PAX7, MYH7, MYOD and MYOGENIN on mRNA level (D). Scale bar: $50 \mu \mathrm{m}$. 


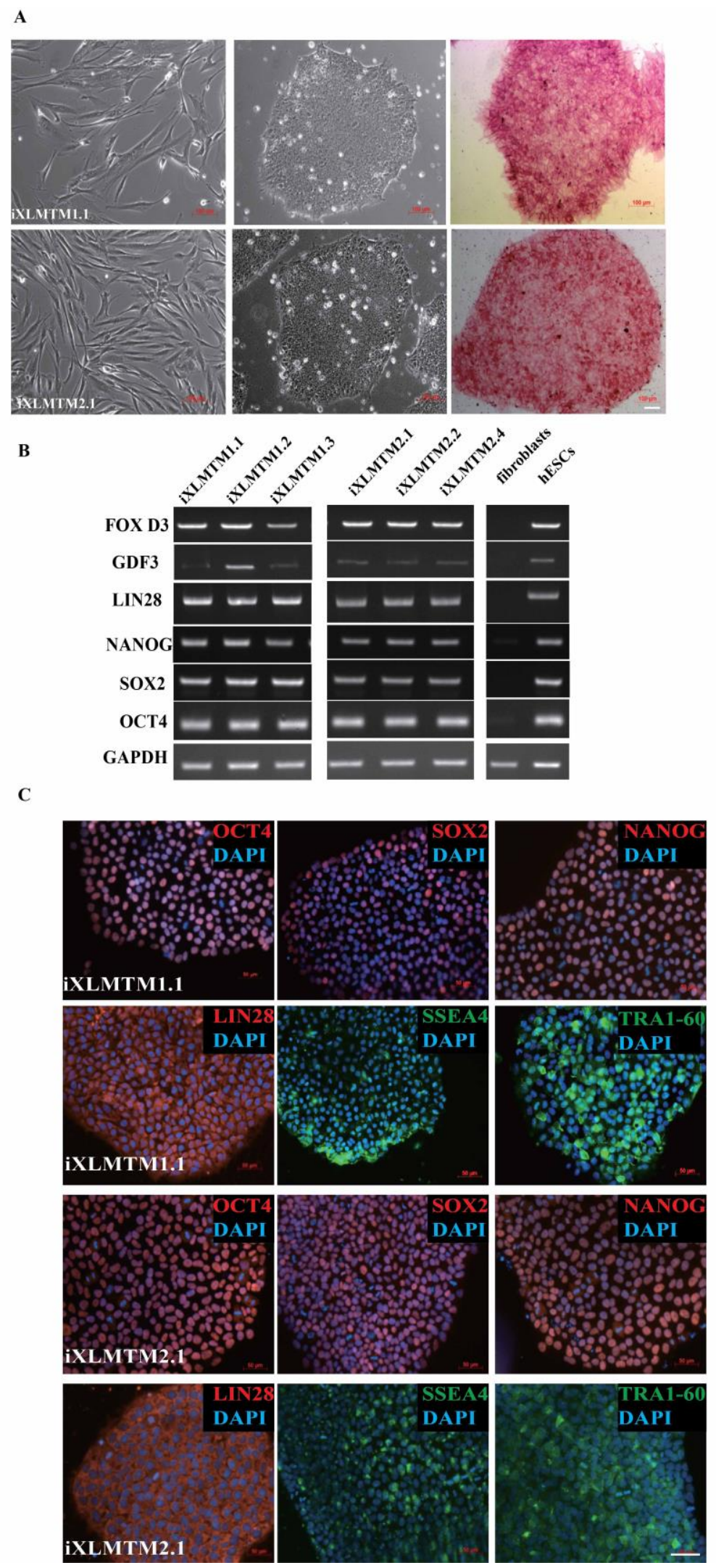

Figure 4. "Proof of pluripotency" of XLMTM patient-specific iPSCs. The iPSCs lines (iXLM1.1, 1.2, 1.3 and iXLM2.1, 2.2 and 2.4) derived from two XLMTM patients with MTM1 mutations c.141_144 del AGAA and c.231+G>A, respectively, were pluripotent, which was demonstrated by the alkaline phosphatase staining (A) and expression of pluripotent-related markers on mRNA (B) and protein level $(\mathbf{C})$. Scale bar: $50 \mu \mathrm{m}$. 
A
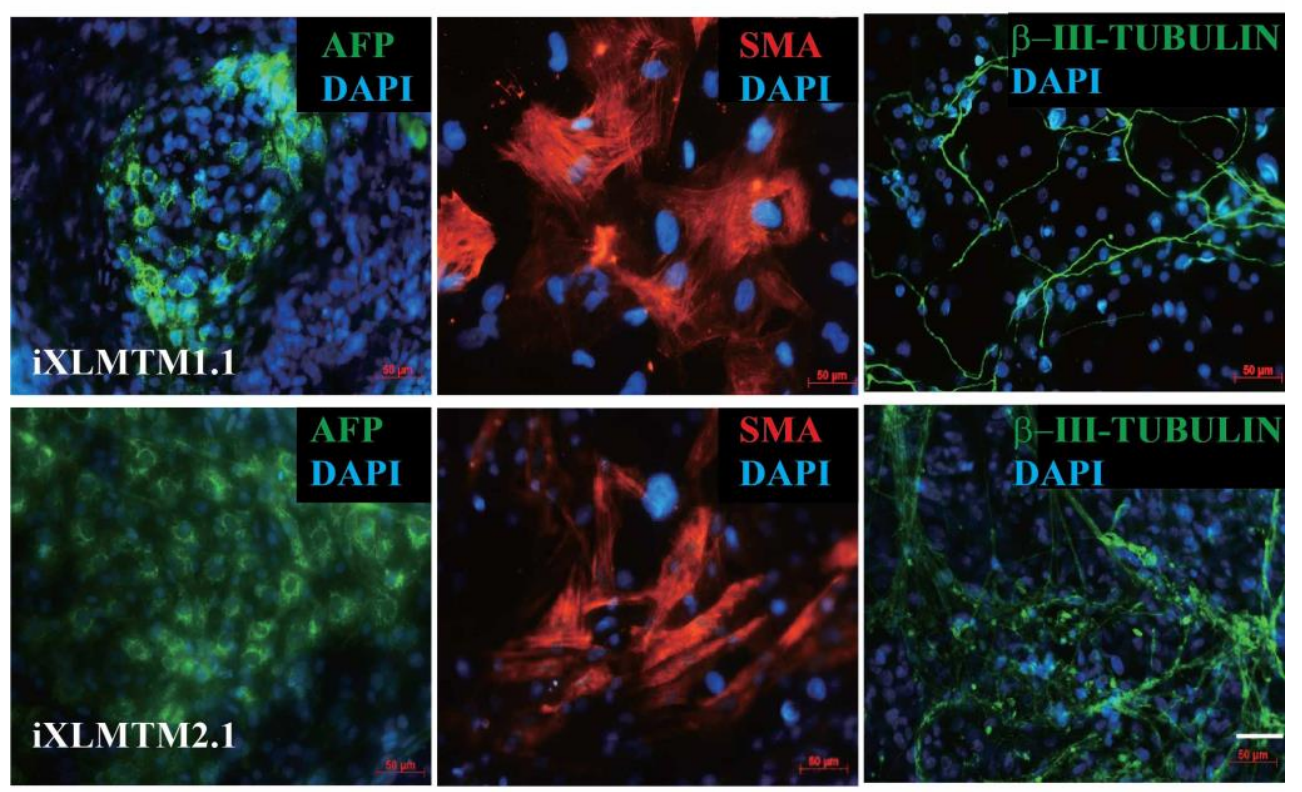

B
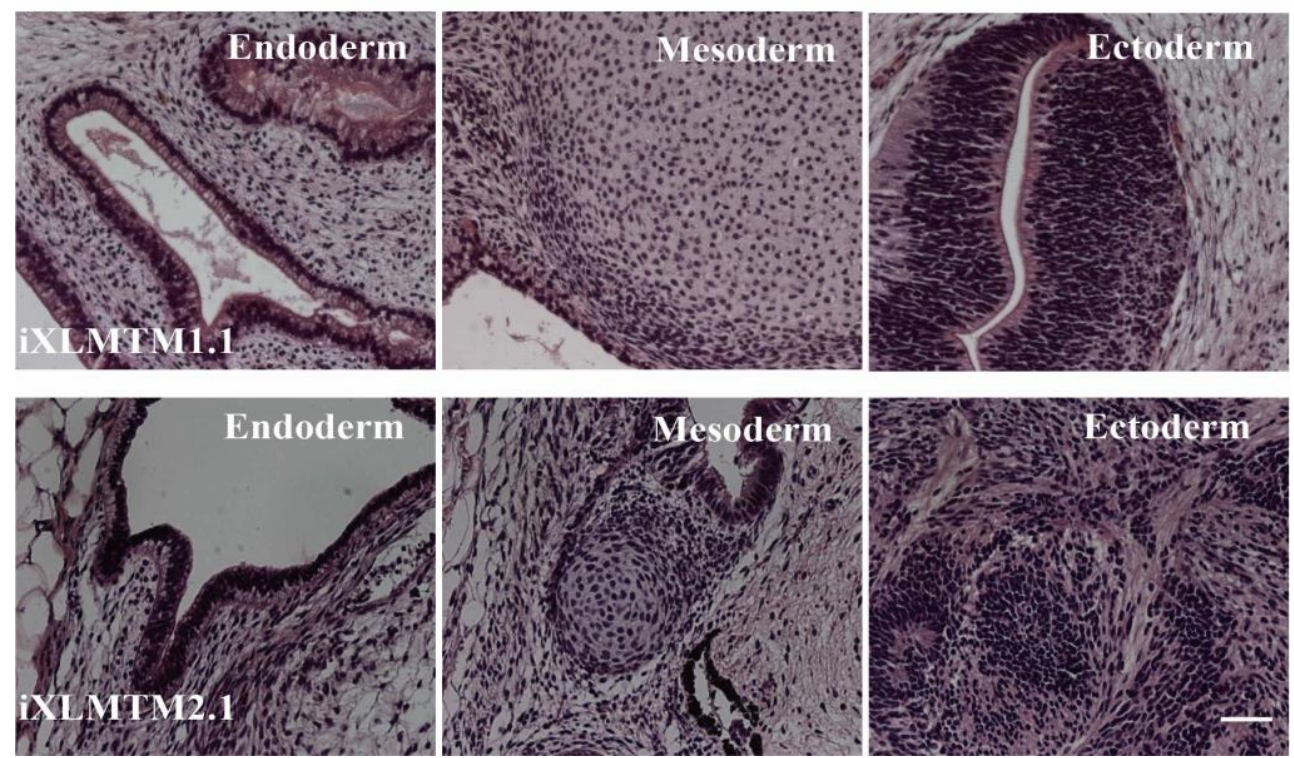

Figure 5. Spontaneous differentiation of XLMTM-iPSCs in vitro and in vivo. These iPSC lines were differentiated into cells or tissues of three germ layers as indicated by expression of endoderm marker AFP, mesoderm marker SMA and ectoderm marker $\beta$-III-TUBULIN in vitro (A) and by teratoma formation with intestinal tissue (endoderm), cartilage (mesoderm) and neural-rosette structure (ectoderm) in vivo (B). Scale bar: $50 \mu \mathrm{m}$.

\section{Discussion}

Skeletal muscle possesses the capacity to regenerate after trauma or disease because of the presence of skeletal-muscle resident stem cells (satellite cells), which are considered as a potential candidate for cell replacement therapy for muscle-related disease. However, there are some limitations to isolate the satellite cells in vitro due to their paucity and 
difficulty to recruit healthy donors. The technique of reprogramming already committed adult cells into iPSCs enables a good approach to generate skeletal muscles in vitro and provides a powerful tool for research in human muscle development and muscle related disease.

Here, we established a new modified skeletal muscle differentiation protocol with small chemical molecules. HiPSCs were exposed to CHIR99021 to induce the mesendoderm progenitor cells, which are committed to myoblasts after bFGF, forskolin and RA treatment. Myoblasts proliferate and differentiate to myocytes in skeletal muscle growth medium; myocytes then fuse with myocytes to form nascent myotubes and mature beating myotubes in skeletal muscle differentiation medium. This direct myogenic differentiation protocol generates skeletal muscles with advanced level of functional maturation, highlighted by the capability of spontaneous contraction. Comparing our differentiation protocol to previous reports, while the modified steps were performed in the absence of culture of embryoid bodies, sorting of progenitor cells and virus transduction, it remains laborious. In order to optimize and shorten the length of the differentiation protocol, myogenic-related genes and proteins would be analyzed at different stages of the differentiation process, revealing the optimal window to exchange medium and apply fresh supplements. Further functional analysis of derived skeletal muscle such as electrophysiological features would be useful for application of this system to dissect the molecular mechanism of muscle disorder. The skeletal muscle cells derived from XLMTM patient-specific iPSCs may model the disease phenotype and help to elucidate the molecular mechanism as a basis for the development of drug targets. Taken together, iPSCs from two XLMTM patients were successfully generated. The iPSCs were pluripotent and were differentiated into cells of three germ layers. To model the muscle phenotype in XLMTM, a myogenic protocol with chemical molecules was established in this study, which directs differentiation of hiPSCs into skeletal muscle in vitro. 


\section{References}

Awaya, T., Kato, T., Mizuno, Y., Chang, H., Niwa, A., Umeda, K., Nakahata, T., and Heike, T. (2012). Selective development of myogenic mesenchymal cells from human embryonic and induced pluripotent stem cells. PLoS One 7, e51638.

Barberi, T., Willis, L.M., Socci, N.D., and Studer, L. (2005). Derivation of multipotent mesenchymal precursors from human embryonic stem cells. PLoS Med 2, e161.

Borchin, B., Chen, J., and Barberi, T. (2013). Derivation and FACS-mediated purification of PAX3+/PAX7+ skeletal muscle precursors from human pluripotent stem cells. Stem Cell Reports $1,620-631$.

Darabi, R., Arpke, R.W., Irion, S., Dimos, J.T., Grskovic, M., Kyba, M., and Perlingeiro, R.C. (2012). Human ES- and iPS-derived myogenic progenitors restore DYSTROPHIN and improve contractility upon transplantation in dystrophic mice. Cell Stem Cell 10, 610-619.

Edwards, M.K., and McBurney, M.W. (1983). The concentration of retinoic acid determines the differentiated cell types formed by a teratocarcinoma cell line. Dev Biol 98, 187-191.

Heckmatt, J.Z., Sewry, C.A., Hodes, D., and Dubowitz, V. (1985). Congenital centronuclear (myotubular) myopathy. A clinical, pathological and genetic study in eight children. Brain 108 ( Pt 4), 941-964.

Ketel, K., Krauss, M., Nicot, A.S., Puchkov, D., Wieffer, M., Muller, R., Subramanian, D., Schultz, C., Laporte, J., and Haucke, V. (2016). A phosphoinositide conversion mechanism for exit from endosomes. Nature 529, 408-412.

Robinson, F.L., and Dixon, J.E. (2006). Myotubularin phosphatases: policing 3-phosphoinositides. Trends Cell Biol 16, 403-412.

Shelton, M., Metz, J., Liu, J., Carpenedo, R.L., Demers, S.P., Stanford, W.L., and Skerjanc, I.S. (2014). Derivation and expansion of PAX7-positive muscle progenitors from human and mouse embryonic stem cells. Stem Cell Reports 3, 516-529.

Tanaka, A., Woltjen, K., Miyake, K., Hotta, A., Ikeya, M., Yamamoto, T., Nishino, T., Shoji, E., Sehara-Fujisawa, A., Manabe, Y., et al. (2013). Efficient and reproducible myogenic differentiation from human iPS cells: prospects for modeling Miyoshi Myopathy in vitro. PLoS One 8, e61540.

Tedesco, F.S., Gerli, M.F., Perani, L., Benedetti, S., Ungaro, F., Cassano, M., Antonini, S., Tagliafico, E., Artusi, V., Longa, E., et al. (2012). Transplantation of genetically corrected human iPSC-derived progenitors in mice with limb-girdle muscular dystrophy. Sci Transl Med 4, 140ra189.

Wallgren-Pettersson, C., and Thomas, N.S. (1994). Report on the 20th ENMC sponsored international workshop: myotubular/centronuclear myopathy. Neuromuscul Disord 4, 71-74. 
Xu, C., Tabebordbar, M., Iovino, S., Ciarlo, C., Liu, J., Castiglioni, A., Price, E., Liu, M., Barton, E.R., Kahn, C.R., et al. (2013). A zebrafish embryo culture system defines factors that promote vertebrate myogenesis across species. Cell 155, 909-921.

Zheng, J.K., Wang, Y., Karandikar, A., Wang, Q., Gai, H., Liu, A.L., Peng, C., and Sheng, H.Z. (2006). Skeletal myogenesis by human embryonic stem cells. Cell Res 16, 713-722. 


\section{Curriculum Vitae}

\section{Personal Contact Information}

Name: Jing Qi

Address: Robert-Koch-Street 38, Room 1203, Göttingen, Germany, 37073

Mobile: 004915772303018

E-mail: qjlvy2006@gmail.com

Gender: Female

Date of birth: March, 27th, 1987

\section{Education}

PhD: Georg-August-University Göttingen and Kings College London, major in Molecular Medicine, July/2013-Oct/2016

Master: Chinese Academy of Science (GIBH), major in Molecular Biology and Biochemistry, Sep/2010-Jun/2013

Bachelor: Sichuan Agriculture University, major in Biology Engineering, Sep/2006-Jun/2010

\section{Instrument Skills}

Stem cells: human and mouse embryonic stem cells, induced pluripotent stem cells (iPSCs), in vitro differentiation, endothelial cells, cardiomyocytes, skeletal muscle cell culture.

Molecular clone: gene expression analysis, PCR, real time-PCR, vector construction, RNAi (siRNA, shRNA), CRISPR/Cas9.

Biochemistry: Western blot, co-IP, FACS, IF, basic epigenetic experiments.

Software: Microsoft Excel, Adobe Photoshop, Adobe Illustrator, etc.

\section{Research and Work Experiences}

July/2013-Now Molecular mechanism study of the muscle disorder disease-using patient-specific iPSCs

- iPSC generation from patient with cardiac or skeletal muscle diseases

- Differentiation of patient-specific iPSCs into functional cardiomyocytes and skeletal muscles

- Autophagy function in cardiomyocytes: regulation on sarcomere structure, uncovering autophagy related cellular pathway

- Using genomic editing tool CRISPR/Cas9 to insert or rescue gene mutation

Sep/2010-Jun/2013 iPSC generation \& mechanisms' basis of the reprogramming process

- Epigenetic modification regulates the key transcription factors to switch on and off the related genes for successful generation of iPSCs

- Time-sequencing for key transcription factors could enhance the process of iPSC generation

\section{Extracurricular Activities}

- Sep/2015 Poster, European Society of Cardiology Congress 2015 
- Nov/2014 Oral presentation, UMG, Göttingen

- Oct/2013 Oral presentation, PhD progress report

\section{Honors and Awards}

- Excellent Student Scholarship, 2013, GIBH.

- Merit Student, National Scholarship, 2009, Sichuan Agricultural University

- Excellent Student Leader 2008, Sichuan Agricultural University

\section{Publication}

1. Chen, J., H. Liu, J. Liu, J. Qi, B. Wei, J. Yang, H. Liang, Y. Chen, J. Chen, Y. Wu, L. Guo, J. Zhu,X. Zhao, T. Peng, Y. Zhang, S. Chen, X. Li, D. Li, T. Wang and D. Pei (2013). H3K9 methylation is a barrier during somatic cell reprogramming into iPSCs. Nat Genet 45(1): 34-42. (impact factor: 29.352)

2. Liu, X., H. Sun, J. Qi, L. Wang, S. He, J. Liu, C. Feng, C. Chen, W. Li, Y. Guo, D. Qin, G. Pan, J. Chen, D. Pei and H. Zheng (2013). Sequential introduction of reprogramming factors reveals a time-sensitive requirement for individual factors and a sequential EMT-MET mechanism for optimal reprogramming. Nat Cell Biol 15(7): 829-838. (impact factors 20.058)

3. Chen, J., L. Guo, L. Zhang, H. Wu, J. Yang, H. Liu, X. Wang, X. Hu, T. Gu, Z. Zhou, J. Liu, J. Liu, H. Wu, S. Q. Mao, K. Mo, Y. Li, K. Lai, J. Qi, H. Yao, G. Pan, G. L. Xu and D. Pei (2013)."Vitamin C modulates TET1 function during somatic cell reprogramming."Nat Genet 45(12): 1504-1509. (impact factor: 29.352)

4. Liu, X., J. Qi, X. Xu, M. Zeisberg, K. Guan and E. M. Zeisberg (2016). Differentiation of functional endothelial cells from human induced pluripotent stem cells: A novel, highly efficient and cost effective method. Differentiation. doi: 10.1016/j.diff.2016.05.004. (impact factor: 3.5). 